Universidade de São Paulo

Instituto de Astronomia, Geofísica e Ciências Atmosféricas

Departamento de Astronomia

Catarina Pasta Aydar

\title{
Diagnostic diagrams for the classification of galactic nuclei: comparison of traditional and alternative diagrams
}

São Paulo 

Catarina Pasta Aydar

\section{Diagnostic diagrams for the classification of galactic nuclei: comparison of traditional and alternative diagrams}

Thesis presented to the Department of Astronomy of the Instituto de Astronomia, Geofísica

e Ciências Atmosféricas of the Universidade de São Paulo as a partial requirement to obtain a Master of Science degree.

Corrected version. The original version is available at the Unit.

Research field: Astronomy

Advisors: Prof. Dr. João E. Steiner,

Prof. Dr. Claudia Lucia Mendes de Oliveira Co-advisor: Dr. Roderik Overzier

São Paulo 

In memory of João E. Steiner. 



\section{Acknowledgements}

Gracias a la vida que me ha dado tanto. Agradeço por tudo porque reconheço que o que tenho é muito.

Agradeço por todo o apoio que me permitiu realizar esse sonho. À minha mãe, Leda Pasta, por estar sempre ao meu lado. Ao meu pai, Mario Manga, por me introduzir a esse fascínio. A meus irmãos e irmã: Caetano, Eduardo, Francisco e Mariana Aydar, amo muito vocês. A minhas avós, meus avôs e minhas tias avós: Yvonne Baptista, Paulina Ferrari, Roque Pasta, Rachid Aydar, Maria Pasta, Edna Ferrari, por serem começos e exemplos. Obrigada Adriana Nunes, Ana Meyer, Bia Aydar, Brisa Aydar, Carla Lavieri, Edna Matsuoka, Iara Pasta, Juliana Pasta, Karen Mizuta, Manuela Aydar, Nicole Torelli, Priscila Aguiar, Sofia Aydar, Tatiana Aydar, Amilcar Pasta, Felipe Aydar, Henrique Pasta, Ivan Pasta, Luiz Sandoval, Marcelo Aydar, Miguel Aydar, Patrick Agostini, Rafael Aydar, Rafael Aguiar Aydar, Vítor Pasta, Ana Deriggi e aos e às demais primos e primas que me acompanharam nessa saga. Obrigada por serem referência em atenção e carinho.

Agradeço a meu orientador João Steiner por tudo o que ele me mostrou e ensinou. E agradeço imensamente a quem me ajudou a atravessar esse luto. À minha orientadora, Claudia Mendes de Oliveira, por todo auxílio. A meu co-orientador, Roderik Overzier, pela integridade e pelo bom humor nas respostas. Aos meus parceiros e parceira de grupo: Daniel May, Patrícia da Silva, Roberto Menezes, Tiago Ricci e um agradecimento especial ao Pedro Henrique Cezar, sem o qual tudo seria mais difícil. A Oli Dors, por me abrir muitas portas. A Alex Carciofi, por ter reconhecido meu potencial humano. Agradeço às e aos pesquisadores Aayush Saxena, Alan Alves Brito, Alessandro Ederoclite, Ana Chies Santos, Anelise Audibert, Angela Krabbe, Anna Feltre, Antônio Kanaan, Beatriz Barbuy, Carlos Eduardo Barbosa, Claus Leitherer, Darshan Kakkad, Eduardo Amôres, Eduardo 
Cypriano, Elysandra Figueredo, Enrique Pérez-Montero, Éverton Fabian, Fabio Herpich, Felipe Fernandes, Francéia Liedtke, Friedrich Anders, Gary Ferland, Gastão Lima Neto, Guilherme Couto, Grazyna Stasińska, Hagai Netzer, Hekatelyne Carpes, Itziar Aretxaga, Jarle Brinchmann, Jorge Barrera-Ballesteros, Jorge Meléndez, Jorge Moreno, Juliana Motter, Laerte Sodré, Leonid Pilyugin, Lisa Kewley, Natacha Dametto, Natalia Vale Asari, Paramita Barai, Patricia Cruz, Patricia Novais, Pedro Beaklini, Rafael Ribeiro, Raquel Santiago Nascimento, Roberto Cid Fernandes, Rodrigo Nemmen, Rogemar Riffel, Rogério Riffel, Sophia Flury, Thaisa Storchi-Bergmann, Thiago Gonçalves, Victoria Reynaldi, Vinicius Placco, William Schoenell e Yaherlyn Díaz. A Gislene Yoko, por me salvar tantas vezes. Ao pessoal do IAG/USP que sempre me amparou: Daniel Moacir Bigardi, José Roberto de Godoy, Ligia Marques, Luciana H. Y. Silveira, Luis Manrique, Marcel Kimura, Marcia Pina, Marcia Ramos, Marco Antonio dos Santos, Maria Teresa Lopes, Richard Lingner, Rosely Souza e Ulisses Castello. À Universidade de São Paulo e ao Instituto de Astronomia, Geofísica e Ciências Atmosféricas. À CAPES, pelo apoio financeiro no começo do mestrado. À FAPESP, pelo apoio financeiro ao projeto $n^{o}$ : 2019/16488-9. E agradeço à banca que avaliará este trabalho pela consideração.

Agradeço aos muitos laços que criei na astronomia, que me ajudam para além de questões profissionais. Muito obrigada ao Ariel Werle, disposto para qualquer pergunta ou palpite. Muito obrigada ao Guilherme Limberg, me impulsionando para além das dificuldades. Muito obrigada ao João Pedro Benedetti, por transformarmos questões em atitudes. Muito obrigada à Luísa Buzzo, por todas as pontes. Muito obrigada à Stela Adduci, sempre tão amorosa. Agradeço muito a Amanda Rubio, Ana Clara Sá, André Figueiredo, Carolina Queiroz, Daiane Seriacopi, Gabriel Fabiano de Souza, G. Gonçalves, Erik Vinicius Lima, Fabio Cafardo, Felipe Navarete, Fernanda Nogueira, Hélio Perottoni, Henrique Lupinari, Ivan Almeida, Jhon Yana, Julia Santos, Jullian Santos, Larissa Takeda, Lia Doubrawa, Lilianne Nakazono, Livia Rocha, Loreany Ferreira, Lucas Siconato, Luís Kadowaki, Marcelo Vincentin, Mirian Castejon Molina, Natalia Andrade, Natalia del Coco, Pablo Araya, Raniere Menezes, Raphael Oliveira, Roberta Duarte, Stefano de Souza, Svetlana Kocheleva, Thainá Sabino, Thaís Silva, Thayse Pacheco e Yuri Abuchaim. Muito obrigada Camila de Sá Freitas, Camila Olave, Claudio Alejandro, Denimara Dias, Eduardo Hartmann, Elismar Löch, Hyoyin Gan, Jana Bogdanoska, Katia Clerici, Luidhy Santana, Marina Bianchin, Marina dal Ponte, Mario Llerena, Mark Armah, Rebeca Batalha, Ro- 
drigo Magalhães, Sander Schouws e Yanna Martins Franco.

Agradeço também aos laços que criei na física e que ainda me fortalecem. Obrigada aos e às docentes incríveis que eu tive e que me nortearam, especialmente Mané Robilotta, Ivã Gurgel, Alex de Lima Barros, Cadu Souza e Rafael Andrade. Obrigada pela força, pela energia e pelos momentos: Bruna Costa, Bruno Nunes, Franklin Rodrigues, Júlio Amando de Barros, Marcelo Janovitch Pereira, Mariana Cipolla, Ramon Vilarino, Renata Biazzi, Theo Motta e Zeca Ribeiro de Carvalho. Muito obrigada por todas as conversas, ajudas, dúvidas e ideias compartilhadas: Adolfo Forti, Barbra Miguele, Bruna Alves, Bruno Minucci, Camila Deodato, Caroline Araujo, Caroline Guandalin, Davi Bastos, Dimy Nanclares, Felipe de Godoy, Felipe Prado, Fernando Passos, Gabriel Nogueira, João Picchetti, José Vitor Borges, Lucas Cornetta, Martim Zurita, Morgana Martins, Naim Comar, Nathalia Rosental, Rafael Bruschi, Rafael Marques, Ramon Barrio, Roberto Baldijão, Sabrina Martins, Teresa Lanna, Tiago Santos, Victor Lellis, Vitoria Dias e Xavier Jr..

Obrigada especialmente às minhas amadas parcerias. Aurora Segre, Daniel Pikman, Felipe Gayotto, Laila Kontic, Lara Ramalho e Mariaji Gandhi, obrigada por estarem comigo. Obrigada por toda conexão a Dani Policastro, Joana Spadaccini, Luísa Moreira, Luísa Torkomian, Pedro Brener, Perla Kindi, Rodrigo Berezovsky, Sofia Fajersztajn, Talissa Ancona Lopez e Zoé Maschio. Agradeço imensamente pelas trocas com Ana Paula Basile, Gabriel Conrado, Emily Chachian, Flora Pappalardo, Frederico Sabanay, João Seckler, Julio Teixeira, Lucas Acher, Marcelo Roubiceck, Marcelo Veras, Matheus Nery e Vitoria Motter. Muito obrigada também a Alexandre Himmelstein, Bia Carvalho, Carolina Alves, Carolina Nemer, Clarice Tavares, Guilherme Melman, Kelwin Marques, Lisa Werebe, Luísa Cytrinovitz, Mariana Amaral, Marina Boh, Marta Cerruti, Nathalya Macchia, Nicole Baumgarten, Renata Mercadante, Roberto Mifano.

Agradeço também por toda ajuda emocional e espiritual. Obrigada a Marta Okamoto, Andrea Perdigão, Valderson de Souza, Maria Ester Massola, Marcos Rojo, Danilo Forghieri, Adriana Miller, Renato Aceto, Kyalene Mesquita e Jon Kabat-Zin.

Por último, agradeço ao Universo, por poder estudar minha origem. 

$X X X I X$

O mistério das cousas, onde está ele? Onde está ele que não aparece Pelo menos a mostrar-nos que é mistério? Que sabe o rio e que sabe a árvore E eu, que não sou mais do que eles, que sei disso? Sempre que olho para as cousas e penso no que os homens pensam delas, Rio como um regato que soa fresco numa pedra.

Porque o único sentido oculto das cousas É elas não terem sentido oculto nenhum, É mais estranho do que todas as estranhezas E do que os sonhos de todos os poetas E os pensamentos de todos os filósofos, Que as cousas sejam realmente o que parecem ser E não haja nada que compreender.

Sim, eis o que os meus sentidos aprenderam sozinhos: As cousas não têm significação: têm existência. As cousas são o único sentido oculto das cousas.

Alberto Caeiro 



\section{Resumo}

Núcleos de galáxias com linhas em emissão em seus espectros podem estar sendo ionizados por formação estelar, atividade associada a um buraco negro supermassivo, choques ou estrelas velhas, quentes e massivas. O objetivo de diagramas diagnósticos é classificar objetos de acordo com sua fonte dominante de energia.

Neste trabalho de mestrado, propomos diagramas diagnósticos alternativos e esquemas de classificação de núcleos de galáxias baseados em razões de linhas em emissão no óptico $([\mathrm{O}$ III $] / \mathrm{H} \beta,[\mathrm{N}$ II $] / \mathrm{H} \alpha,[\mathrm{S}$ II $] / \mathrm{H} \alpha$ e $[\mathrm{O} \mathrm{I}] / \mathrm{H} \alpha)$. Esses esquemas são capazes de classificar núcleos de galáxias como regiões de formação estelar (regiões H II ), Seyferts (núcleos ativos de galáxias com alta ionização) ou LINERs (regiões nucleares com linhas em emissão e baixa ionização). Três dos diagramas alternativos propostos nessa dissertação usam todas as razões de linhas em emissão dos três diagramas diagnósticos tradicionais em combinações lineares, de forma a evitar alguns conhecidos problemas de classificação ambígua. Outro dos novos diagramas diagnósticos apresentados oferece uma abordagem mais econômica, utilizando apenas linhas em emissão que estejam próximas com relação ao comprimento de onda, de forma a evitar erros no fluxo devido a efeitos de calibração e correção por avermelhamento ou a necessidade de ampla cobertura espectral. Também sugerimos uma nova equação para descrever a abundância de nitrogênio com relação ao oxigênio, que pode ser usada como parâmetro para modelos de fotoionização de regiões H II.

Para testar a robustez dos diagramas alternativos, nós os comparamos aos diagramas diagnósticos mais tradicionais. Extraímos uma amostra de núcleos de galáxias do Sloan Digital Sky Survey Main Galaxy Sample (com redshift $z<0.016$ e incerteza com relação ao logaritmo das razões de linhas de $\epsilon<0.1$ dex) e a aplicamos em diversos esquemas de classificação baseados nos diagramas diagnósticos tradicionais do óptico para decidir onde 
as fronteiras dos nossos novos esquemas de classificação deveriam estar.

Depois de realizar esses estudo, nós investigamos modelos teóricos de fotoionização de nebulosas gasosas por formação estelar e por espectro de lei de potências típico de AGNs. Nós usamos os códigos Starburst99 para gerar a distribuição de energia espectral de populações estelares e CLOUDY para performar os cálculos de transferência radiativa. Nós mostramos que as principais propriedades que determinam a posição de um objeto nos diagramas diagnósticos são a metalicidade, o parâmetro de ionização e o quão "duro" (maiores energias) o campo de radiação ionizante é. Concluímos que modelos que consideram uma única e constante densidade não são capazes de descrever a complexidade de nebulosas gasosas fotoionizadas e revisamos algumas das abordagens mais refinadas para se modelar núcleos ativos de galáxias.

Assim, este trabalho oferece uma revisão sobre a classificação e a modelagem de núcleos de galáxias, além de propor novas ferramentas - quatro diagramas diagnósticos alternativos com seus esquemas de classificação e uma nova equação para a abundância de nitrogênio em regiões de formação estelar - que nos ajudem a melhor compreender as nebulosas fotoionizadas encontradas nos centros da maioria das galáxias próximas. Nos próximos anos, será possível aplicar as técnicas de diagnóstico no óptico em repouso discutidas nessa dissertação pela primeira vez para um grande número de galáxias em altos redshifts, usando as mesmas linhas em emissão desviadas para o infravermelho próximo e médio. 
As regiões centrais de galáxias por vezes apresentam um brilho muito mais intenso do que todo o restante da galáxia. Sabemos que nessa região reside um buraco negro supermassivo, mas também pode haver gás e estrelas. O objetivo deste trabalho é de aperfeiçoar as ferramentas utilizadas para classificar a atividade no núcleo de galáxias. Queremos determinar de forma mais precisa se o que está gerando o brilho na região nuclear de cada galáxia é atividade estelar ou associada ao crescimento do buraco negro central.

Para isso, utilizamos uma amostra de 4192 observações espectrais de núcleos de galáxias próximas e propomos diagramas que nos permitam separar aqueles que são ionizados por estrelas daqueles ionizados por acreção de matéria por um buraco negro supermassivo. Além disso, construímos modelos do gás ionizado por essas duas possíveis fontes de energia para melhor compreender a utilidade dos diagramas que estamos propondo.

Descrevemos esse estudo com uma revisão acerca de classificação de núcleos de galáxias, e propomos novas ferramentas que colaborem para o aperfeiçoamento desse estudo. 



\section{Abstract}

Galactic nuclei with emission lines in their spectra can be ionized by star-formation, activity of a supermassive black hole, shocks, or hot old massive stars. The purpose of diagnostic diagrams is to classify objects according to their main power source.

In this work, we propose alternative diagnostic diagrams and classification schemes for galactic nuclei based on optical emission-line ratios $([\mathrm{O} \mathrm{III}] / \mathrm{H} \beta,[\mathrm{N}$ II $] / \mathrm{H} \alpha,[\mathrm{S}$ II $] / \mathrm{H} \alpha$, and $[\mathrm{O} \mathrm{I}] / \mathrm{H} \alpha$ ). With these schemes, we are able to classify galactic nuclei as star-forming regions (H II regions), Seyferts (high ionization active galactic nuclei) or LINERs (LowIonization Nuclear Emission-line Regions). Three of the alternative diagrams presented in this work use all the emission line ratios of the three standard diagnostic diagrams in linear combinations, therefore avoiding some well-known ambiguity issues. Another of the diagnostic diagrams that we propose offers a more economic approach by using only emission lines that are near in wavelength, avoiding flux errors due to calibrations and reddening effects or the need for spectra covering a large wavelength range. We also suggest a new equation to describe the abundance of nitrogen with regard to oxygen which can be used as input for $\mathrm{H}$ II region photoionization codes.

In order to test the robustness of the alternative diagrams, we compare them to the standard diagnostic diagrams. We extract a sample of galactic nuclei from the Sloan Digital Sky Survey Main Galaxy Sample (redshift $z<0.016$ and uncertainty of the logarithm of all the emission line ratios $\epsilon<0.1 \mathrm{dex}$ ) and apply several classification schemes based on the standard optical diagnostic diagrams to decide where the boundaries in our new classification schemes should lie.

After this empirical study, we investigate theoretical models of gaseous nebulae photoionization from star-formation and power-law spectra typical for AGNs. We use STAR- 
BURST99 to generate the spectral energy distribution of stellar populations and CLOUDY to perform the radiative transfer calculations. We show that the main properties that determine the position of an object in the diagnostic diagrams are the metallicity, the ionization parameter, and the hardness of the ionization field. However, we conclude that models with a single constant density are not able to describe the complexity of photoionized gaseous nebulae, and we review some of the more refined approaches to model active galactic nuclei.

Hence, this work offers a review of the classification and modeling of galactic nuclei, and we propose new tools - four alternative diagnostic diagrams with their classification schemes and a new equation for the nitrogen abundance in star-forming regions - to help better understand the photoionized nebulae found in the centers of most nearby galaxies. In the coming years, it will become possible to apply the rest-frame optical diagnostic techniques discussed in this thesis for the first time to larger numbers of galaxies at high redshifts, using the same emission lines redshifted to the near- and mid-infrared. 


\section{List of Figures}

1.1 Fig. 1 from $\mathrm{Ho}(2008)$ with typical spectra of emission-line nuclei. . . . . . 29

1.2 Scheme of a gaseous nebula photoionized by a central source. . . . . . . . . 32

1.3 Image of an AGN. . . . . . . . . . . . . . . . . . . . . . . 34

1.4 Fig. 1.1 from Beckmann and Shrader (2012) with the unified model of AGNs. 36

1.5 Fig. 3 from Heckman and Best (2014) with the radiative- and jet-mode AGNs. 38

1.6 Image of a star forming region. . . . . . . . . . . . . . . . . . 40

1.7 Fig. 1 from Brown et al. (2000) adapted by Paola Marigo. Stellar evolutionary tracks in the Hertzsprung-Russell diagram, indicating HOLMES. . 44

1.8 Fig. 5 from Baldwin, Phillips \& Terlevich (1981), presenting the BPT diag-

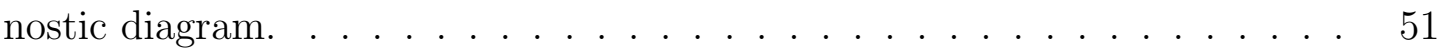

1.9 Figs. 2 and 6 from Veilleux and Osterbrock (1987) presenting the VO-S and VO-O diagrams. . . . . . . . . . . . . . . . . . . . 54

3.1 Standard diagnostic diagrams. . . . . . . . . . . . . . 68

3.2 Standard diagnostic diagrams with all analyzed classification schemes. . . . 72

3.3 Standard diagnostic diagrams with the classification scheme of Ho, Filippenko \& Sargent (1997). . . . . . . . . . . . . . . . . . . . . . 76

3.4 Standard diagnostic diagrams with the classification scheme of Kewley et al.

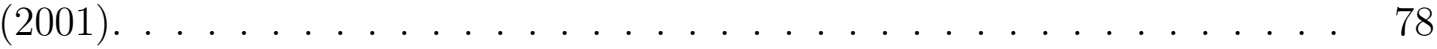

3.5 Standard diagnostic diagrams with the classification scheme of Kauffmann et al. $(2003) \ldots \ldots \ldots \ldots \ldots$

3.6 Standard diagnostic diagrams with the classification scheme of Kewley et al.

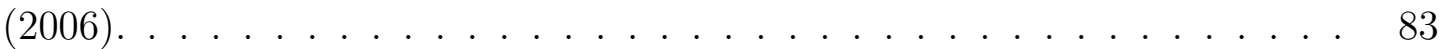


3.7 Standard diagnostic diagrams with the classification scheme of Stasińska et al. $(2006) \ldots \ldots \ldots \ldots \ldots \ldots$. . . . . . . . . . . . . . . . . . . . . .

3.8 Standard diagnostic diagrams with the classification scheme of Stasińska et al. (2006) considering only the nitrogen line ratio. . . . . . . . . . . 87

3.9 Standard diagnostic diagrams with the classification scheme of Schawinski et al. $(2007) \ldots \ldots \ldots$

3.10 Standard diagnostic diagrams with the classification scheme of Cid Fernandes et al. (2010). . . . . . . . . . . . . . . . . . . 91

3.11 Standard diagnostic diagrams with the classification scheme of Meléndez et al. $(2014) \ldots \ldots \ldots \ldots$

3.12 Balmer decrement. . . . . . . . . . . . . . . . . 112

3.13 Alternative diagnostic diagram with the sum of the emission line ratios in the axes. . . . . . . . . . . . . . . . . . 115

3.14 Standard diagnostic diagrams with the classification scheme from Fig. 3.13, 116

3.15 Alternative diagnostic diagram with the sum of the oxygen line ratios and the difference between nitrogen and sulfur line ratios. . . . . . . . . . . . 117

3.16 Standard diagnostic diagrams with the classification scheme from Fig. 3.15, 118

3.17 Alternative diagnostic diagram with the difference between the oxygen line ratios and the sum of nitrogen and sulfur line ratios. Classification scheme according to Heckman $(1980)$. . . . . . . . . . . . . . . . . . 120

3.18 Standard diagnostic diagrams with the classification scheme from Fig. 3.17, 121

3.19 Alternative diagnostic diagram with the difference between the oxygen line ratios and the sum of nitrogen and sulfur line ratios.

3.20 Standard diagnostic diagrams with the classification scheme from Fig. 3.19, 123

3.21 Alternative diagnostic diagram with the nitrogen and sulfur line ratios. Classification scheme separating star-forming nuclei, Seyferts and LINERs. 125

3.22 Standard diagnostic diagrams with the classification scheme from Fig. 3.21, 127

3.23 Alternative diagnostic diagram with the nitrogen and sulfur line ratios as axes. Classification scheme separating star-forming nuclei and AGNs. . . . 128

3.24 Standard diagnostic diagrams with the classification scheme from Fig. 3.23, 129

3.25 Alternative diagnostic diagram introduced by Dopita et al. (2013) . . . . . . 131

3.26 Starburst99 spectra of the extreme metallicities. . . . . . . . . . . . 139 
3.27 StARBURST99 spectra in comparison with solar metallicity.

3.28 Fig. 1 from Feltre, Charlot \& Gutkin (2016) with the comparison of stellar and AGN spectra. . . . . . . . . . . . . . . . . . 143

3.29 BPT diagrams with models for nitrogen abundance. . . . . . . . . . . . . 160

3.30 Alternative diagnostic diagrams with models for nitrogen abundance. . . . 161

3.31 BPT diagram with grid of star-forming objects. . . . . . . . . . . . . 163

3.32 VO-S diagram with grid of star-forming objects. . . . . . . . . . . . 164

3.33 Alternative diagnostic diagram from Fig. 3.21 with grid of star-forming

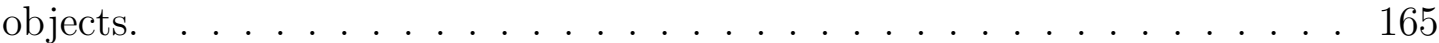

3.34 Alternative diagnostic diagram from Fig. 3.25 with grid of star-forming nuclei.166

3.35 BPT diagram with star-forming and AGN models. . . . . . . . . . . . 167

3.36 VO-S diagram with star-forming and AGN models. . . . . . . . . . . . 168

3.37 VO-O diagram with star-forming and AGN models. . . . . . . . . . . . . 169

3.38 Alternative diagnostic diagram from Fig. 3.21 with star-forming and AGN models. . . . . . . . . . . . . . . . . . . 170

3.39 Alternative diagnostic diagram from Fig. 3.13 with star-forming and AGN models. ........................... . . . . . . . . . . .

3.40 Alternative diagnostic diagram from Fig. 3.19 with star-forming and AGN models.

A.1 Fig. 4 from Binette et al. (1996) with geometrical distributions of matterand ionization-bounded components. . . . . . . . . . . . . . . . . 214

A.2 Fig. 1 from Dopita et al. (2002) with the dusty, radiation pressure-dominated photoionization model.

B.1 Nitrogen line ratio versus redshift. . . . . . . . . . . . . . . . . 225 



\section{List of Tables}

3.1 Summary of the classification schemes discussed in Section 3.1.1 . . . . . 73

3.2 Classification scheme proposed by Ho, Filippenko \& Sargent (1997) . . . . . 75

3.3 Results of the classification schemes applied to our sample. . . . . . . . 9 95

3.4 The main parameters and values of the photoionization models in this work. 156 



\section{Contents}

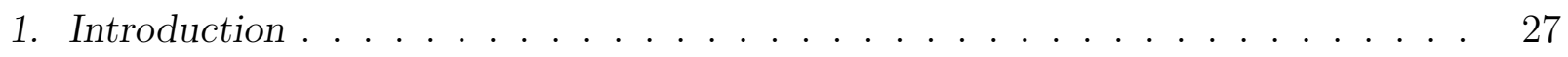

1.1 Galactic nuclei . . . . . . . . . . . . . . . . . . . . . . 28

1.1.1 Gaseous nebulae ...................... 30

1.1.2 Active galactic nuclei . . . . . . . . . . . . . 33

1.1.3 Stellar activity .................... 40

1.1.3.1 Young OB stars . . . . . . . . . . . . . . . . . . 41

1.1.3.2 Wolf-Rayet . . . . . . . . . . . . . 42

1.1.3.3 Hot Low-Mass Evolved Stars . . . . . . . . . . . . . . . 43

1.2 Diagnostic diagrams . . . . . . . . . . . . . . . . . 44

1.2.1 Historical review . . . . . . . . . . . . . . 47

1.2.1.1 Baldwin, Phillips \& Terlevich 1981 . . . . . . . . . . 49

1.2.1.2 Veilleux \& Osterbrock $1987 \ldots \ldots$. . . . . . . 52

1.2 .2 Other wavelengths . . . . . . . . . . . . 56

1.3 Motivation .......................... 59

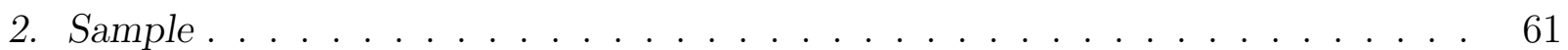

2.1 Survey requirements . . . . . . . . . . . . . . . . . . . 61

2.2 Sloan Digital Sky Survey . . . . . . . . . . . . . . . . . . 63

2.3 Specifications of the sample used in this thesis . . . . . . . . . . . 64

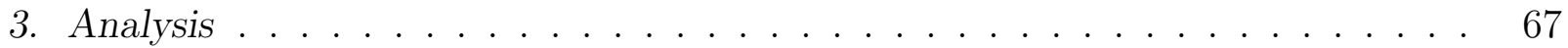

3.1 Traditional diagnostic diagrams . . . . . . . . . . . . . . 68

3.1.1 Object classification ..................... 71

3.1.1.1 Ho, Fillipenko \& Sargent 1997 . . . . . . . . . . . . . 73 
3.1.1.2 Kewley et al. $2001 \ldots \ldots \ldots$. . . . . . . . 77

3.1.1.3 Kauffmann et al. 2003 . . . . . . . . . . . . . 79

3.1.1.4 Kewley et al. $2006 \ldots \ldots$. . . . . . . . . 81

3.1.1.5 Stasinska et al. $2006 \ldots \ldots$. . . . . . . . . 85

3.1.1.6 Schawinski et al. $2007 \ldots \ldots$. . . . . . . . . . . 88

3.1.1.7 Cid Fernandes et al. 2010 . . . . . . . . . . . . . 91

3.1.1.8 Meléndez et al. $2014 \ldots \ldots$. . . . . . . . . . . . 92

3.1.1.9 Discussion . . . . . . . . . . . . . . . . . . . . 93

3.1 .2 Transition objects . . . . . . . . . . . . . . . . 97

3.1 .3 Misclassification . . . . . . . . . . . . . . . . 102

3.2 Alternative diagnostic diagrams . . . . . . . . . . . . . . 110

$3.2 .1 \quad([\mathrm{O} \mathrm{III}] / \mathrm{H} \beta) \times([\mathrm{O} \mathrm{I}] / \mathrm{H} \alpha)$ vs. $([\mathrm{N} \mathrm{II}] / \mathrm{H} \alpha) \times([\mathrm{S} \mathrm{II}] / \mathrm{H} \alpha) \ldots \ldots 113$

$3.2 .2([\mathrm{O} \mathrm{III}] / \mathrm{H} \beta) \times([\mathrm{O} \mathrm{I}] / \mathrm{H} \alpha)$ vs. $([\mathrm{N} \mathrm{II}] / \mathrm{H} \alpha) /([\mathrm{S} \mathrm{II}] / \mathrm{H} \alpha) \ldots \ldots 116$

$3.2 .3([\mathrm{O} \mathrm{III}] / \mathrm{H} \beta) /([\mathrm{O} \mathrm{I}] / \mathrm{H} \alpha)$ vs. $([\mathrm{N} \mathrm{II}] / \mathrm{H} \alpha) \times([\mathrm{S} \mathrm{II}] / \mathrm{H} \alpha) \ldots \ldots 119$

$3.2 .4([\mathrm{~S} \mathrm{II}] / \mathrm{H} \alpha)$ vs. $([\mathrm{N} \mathrm{II}] / \mathrm{H} \alpha) \ldots \ldots \ldots \ldots \ldots \ldots$

$3.2 .5([\mathrm{O} \mathrm{III}] / \mathrm{H} \beta)$ vs. $([\mathrm{N} \mathrm{II}] /[\mathrm{S} \mathrm{II}]) \ldots \ldots \ldots \ldots \ldots \ldots$

3.2 .6 Summary . . . . . . . . . . . . . . . . . . 132

3.3 Theoretical modeling . . . . . . . . . . . . . . . . . . . . . 132

3.3.1 Stellar spectral energy distribution . . . . . . . . . 135

3.3 .2 Photoionization models . . . . . . . . . . . . . . . . 140

3.3.3 Nitrogen abundance . . . . . . . . . . . . . . . . . . . . 155

3.3.4 Application to diagnostic diagrams . . . . . . . . . . . 162

4. Conclusions and Perspectives . . . . . . . . . . . . . . . 175

4.1 Conclusions . . . . . . . . . . . . . . . . . 175

4.2 Perspectives . . . . . . . . . . . . . . . . . . . . 178

Bibliography . . . . . . . . . . . . . . . . . . . 181

$\begin{array}{ll}\text { Appendix } & 209\end{array}$

A. More complex models for AGN . . . . . . . . . . . . . . . . . . . 211

A.1 Matter- and ionization-bounded clouds . . . . . . . . . . . . 212 
A.2 Local optimally emitting clouds . . . . . . . . . . . . . . 215

A.3 Shocks . . . . . . . . . . . . . . . . . . . . . . . . . . . . 217

A.4 Dusty, radiation pressure-dominated photoionization $\ldots \ldots \ldots . .220$

B. Aperture effect . . . . . . . . . . . . . . . . . . . 225 

Chapter 1

\section{Introduction}

A considerable fraction of the galaxies in our Universe presents narrow emission lines in their spectra (Veilleux and Osterbrock, 1987). Emission lines are useful to better understand these astronomical objects and their evolution with regard to parameters such as their excitation source, metallicity, ionization parameter, interstellar medium (ISM) pressure, and electron density (Kewley et al., 2019, defined in Section 3.3.2).

In the center of every evolved massive galaxy resides a supermassive black hole (SBMH, Magorrian et al., 1998; $\mathrm{Ho}, 2008$; Schawinski et al., 2010). In some fraction of those, the central region hosts a high surface brightness compact component indicative of non-stellar nuclear activity. Other galaxies, like the Milky Way, do not show particularly luminous activity in their nucleus, but the orbits of stars near the nucleus indicate the presence of a large, compact and dark mass: a SMBH (Ghez et al., 1998, 2008, Gillessen et al., 2009; Genzel et al., 2010; Gravity Collaboration, 2018). In the case of galactic nuclei showing activity, their spectra reveal forbidden emission lines that indicate that the gas in that region is being ionized. The relative strengths of the emission lines (including hydrogen recombination lines) can be a good indicator of the main astrophysical phenomenon that is causing the excitation of the gas. The main candidates for the power source in galactic nuclei are accreting SMBHs (denominated Active Galactic Nuclei; AGNي? ), stars or starformation, and shocks (the latter are a consequence of the former processes).

The main goal of this work is to propose alternative diagnostic diagrams that can help us to better constrain the dominant ionization source acting on galactic nuclei, distinguishing

\footnotetext{
${ }^{1}$ We will refer to Active Galactic Nucleus in singular as AGN and Active Galactic Nuclei in plural as AGNs. Though in Latin the singular form ends with "s" and the plural form does not, writing AGNs for the plural follows from our definition of AGN for the singular.
} 
mainly star-forming nuclei from AGNs (high- or low-ionization) using easily observable emission line features from the optical spectra of the central region of galaxies.

In this Section, we will present the main astrophysical processes that can contribute to photoionization in the center of galactic nuclei. The general of gaseous nebulae will be given in Section 1.1, and AGNs and stellar processes will be detailed in Sections 1.1.2 and 1.1.3, respectively. Then, in Section 1.2 we will present the diagnostic diagrams that have been widely used in the literature to classify galactic nuclei according to their dominant power source. In Section 1.3 we will summarize the main motivation behind our study of alternative diagnostic diagrams that form the central part of this thesis. Our data is described in Section 2, The analysis of this work is presented in Section 3, with the traditional diagnostic diagrams in Section 3.1, the alternative diagnostic diagrams in Section 3.2, and the theoretical modeling in Section 3.3. Finally, we present our conclusions and future perspectives in Section 4. Appendix A presents more complex models of AGNs and Appendix $\mathrm{B}$ contains a brief discussion on aperture effects.

\subsection{Galactic nuclei}

The nuclei of massive galaxies are very interesting environments, since they host the central SMBH (Magorrian et al., 1998; Kormendy and Ho, 2013; Ho, 2008) and can also be rich in gas and stars. These regions are crucial for the study of the evolution of the SMBH and how its development is connected to the evolution of the host galaxy itself Ferrarese and Merritt, 2000; Gebhardt et al., 2000; Kewley et al., 2006). Therefore, it is important that we have the tools to characterize these nuclear regions of galaxies, and can distinguish the main sources of ionization acting on these extreme environments.

The excitation of nebular gas in and around a galactic nucleus can be explained by two main mechanisms: stellar photoionization or photoionization by a hard radiation field, such as that produced by the accretion disk around a black hole (Ho, 2008, Heckman and Best, 2014). Both mechanisms can generate shock waves that also ionize the medium, but such shocks are unlikely the main source of excitation in galactic nuclei (Halpern and Steiner, 1983; Yan and Blanton, 2012). In this work, we will focus mainly on the differentiation of objects photoionized by massive stars in H II regions (regions of star formation; see Section 1.1.3.1) and AGNs. The latter can be further differentiated according to their 


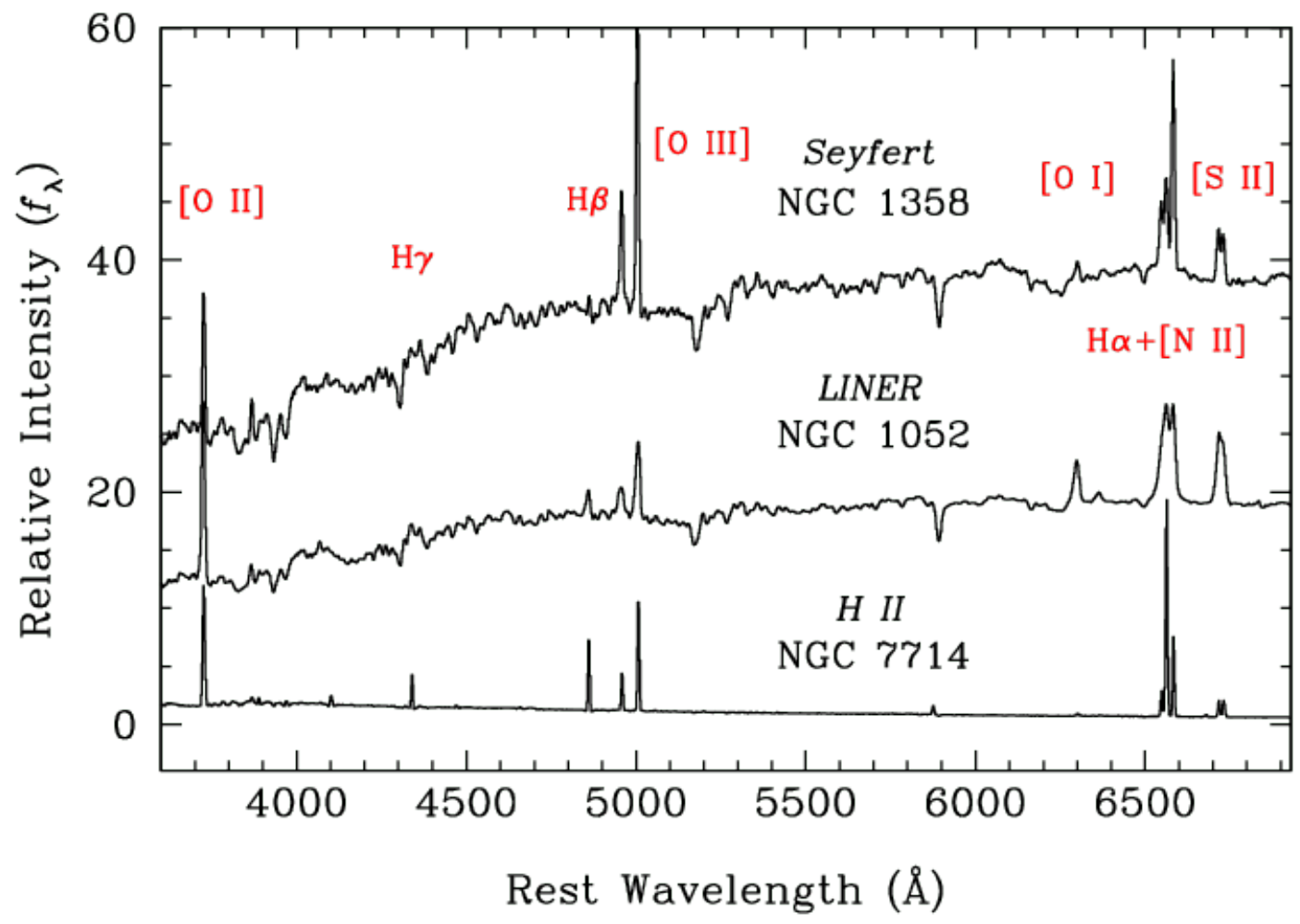

Figure 1.1: Figure 1 from Ho (2008), showing the sample optical spectra of the various classes of emissionline nuclei, with prominent Balmer and forbidden emission lines identified.

ionization levels into Seyferts and Low Ionization Nuclear Emission-line Regions (LINERs, see Section 1.1.2). Figure 1.1 shows typical optical spectra of each of these types of galactic nuclei, with the following significant differences: the shape and intensity of the emission lines and the shape of the continuum. Given these differences, determining criteria for spectral classification is important for better understanding which is the main ionization source acting on each observed galactic nuclei.

It is important to emphasize that the diagnostic diagrams that are studied in this work focus on determining the dominant power source in each nucleus, but that does not mean that the observed nucleus has only one stellar or non-stellar process occurring. A realistic galactic nucleus consists of different regions or components each with its own relevant ionization mechanisms. A popular paradigm of galaxy formation models is that (massive) early-type galaxies undergo an evolutionary sequence from star formation via nuclear activity to quiescence (e.g., Schawinski et al., 2007, and references therein). In that scenario, AGNs and star formation are tightly connected. The same mechanism that supplies gas for star formation, may supply a small fraction of gas to the very central 
region. Furthermore, star formation can feed the massive black hole via supernova winds and mass-loss or result in stellar orbits that are too close from the gravitational influence of the black hole (e.g., Wild et al., 2010). As for the AGNs, their feedback can be negative or positive, meaning that they can suppress star formation by the expulsion of gas (e.g., Gabor et al., 2010) or they can enhance star formation by the accumulation of gas that was removed from the nuclei and condensed in the circumnuclear regions (see Cresci et al., 2015, and references therein). With regard to the contribution of shocks, these can originate from stellar winds associated with Wolf-Rayet (WR) stars (e.g., Simpson et al., 2007), supernovae and wind-blown bubbles from massive stars (e.g., Strickland and Heckman, 2009), starburst winds (e.g., Heckman et al., 2017), and from outflows associated with the accretion of material into SMBHs, the so called AGN-driven winds (see Veilleux et al. 2005).

Therefore, star formation, AGN, and shocks are typically found to coexist (Kewley et al., 2019), and many AGNs are surrounded by circumnuclear regions of star formation or nuclear starbursts (Cid Fernandes et al., 2001; Veilleux et al., 2003, 2005; Dors et al. 2008). In the next Section, we will describe the similarities and differences between gaseous nebulae photoionized by stars and AGNs.

\subsubsection{Gaseous nebulae}

Many galaxies show nebular emission lines in the spectra of their nuclei. They can be photoionized by hot stars (that can be in the form of a single giant or several H II regions, or older stellar populations). However, in other cases, the nuclei emit lines from ions with a range of ionization levels that cannot be produced by $\mathrm{H}$ II regions alone, extending to much higher energies than the spectra of hot stars. This non-stellar photoionization is probably due to an accretion disk that surrounds a SMBH or, alternatively, by the relativistic particles and magnetic fields around the SMBH (Osterbrock and Ferland, 2006). These objects are referred to as AGNs, and there are many different sub-classes defined, some of which have a historical or observational basis while other indicate real physical differences. In this work, we will focus mainly on Seyferts (high-ionization) and LINERs (low ionization, Heckman 1980).

The emission-line spectra of galactic nuclei are an important probe of the gaseous component of galaxies, giving more information the chemical evolution, the star formation 
history (SFH) and other important aspects of their evolution (Yan and Blanton, 2012). The basic physical principles that define the structure of gaseous nebula ionized by AGNs or H II regions are very similar. Even though, due to the larger proportion of high-energy photons in the AGNs, there are crucial differences between the gaseous nebulae photoionized by stellar and non-stellar processes (Osterbrock and Ferland, 2006).

Photoionized nebulae display a thermal equilibrium, in which a balance exists between heating (by photoionization and photoelectric heating by dust grains) and cooling (by recombination and radiation from the nebulae). The power sources (of stellar origin or not) provide ultraviolet (UV) radiation and photons with energies higher than $13.6 \mathrm{eV}$ (the ionization potential of $\mathrm{H}$ ) will heat the gas by liberating electrons, while the gas also cools by the radiative decay of collisionally excited lines from ions such as $\mathrm{O}^{++}, \mathrm{O}^{+}$, $\mathrm{N}^{+}$, and many others, transforming thermal energy in radiative energy that we observe in the spectra. This thermal balance typically lies around an electron temperature of $10^{4} \mathrm{~K}$, depending on the radiation field, the chemical abundance of the medium, and the ionization parameter. The degree of ionization at each point of the nebula is defined by the equilibrium between photoionization and the recombination of thermal electrons by the ions (Osterbrock and Ferland, 2006, Pagel, 2009).

The collisions between thermal electrons and ions excite the low-lying energy levels of such ions. Most of these collisionally excited lines are from 'forbidden' transitions, transitions with such low transition probability that they are not reproduced in laboratories. However, at photoionized nebulae with typically low densities of nebulae (typically 10 to $10^{4} \mathrm{~cm}^{-3}$ ), the collisional de-excitation is so slow that a photon is eventually emitted in a radiative de-excitation, and thus forbidden lines are frequently very strong in their spectra (Osterbrock and Ferland, 2006, Pagel, 2009).

The observed helium and hydrogen lines are formed by similar recombination processes, where recombinations occur to excited levels and these excited atoms decay to lower levels by radiative transitions until they reach the ground level. In the case of hydrogen, these are the H I Balmer, Paschen, and Bracket lines that are observed in all gaseous nebulae spectra, with each of these series being associated to different wavelength ranges Osterbrock and Ferland, 2006; Pagel, 2009).

Thus, the ionization equilibrium at each point in the nebula is given by the balance between photoionization and recombination of electrons with the ions, and also by the 


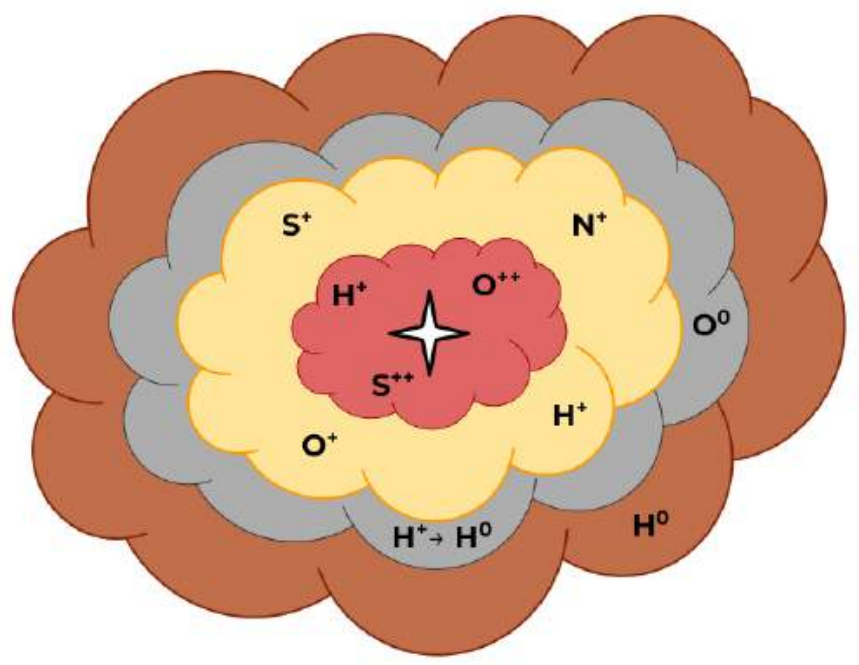

Figure 1.2: Scheme of a gaseous nebula photoionized by a central source. The central white cross represents the ionizing source, that can be an AGN or a star/stellar population. The red cloud that surrounds the central source will produce high-ionization emission lines as $\mathrm{H}^{+}, \mathrm{O}^{++}$, and $\mathrm{S}^{++}$. The yellow cloud that surrounds the red cloud will produce intermediate-ionization emission lines as $\mathrm{H}^{+}, \mathrm{O}^{+}, \mathrm{S}^{+}$, and $\mathrm{N}^{+}$. Both the red and the yellow clouds produce H II , therefore being denominated H II region. The gray cloud represents the partially ionized zone, where the transition between the ionized and neutral hydrogen occurs. Low-ionization emission lines as $\mathrm{O}^{+}$are produced in this gray region. The brown cloud represents the neutral $\mathrm{H}$ I region, where $\mathrm{H}^{0}$ is emitted. This simplistic scheme does not represent density variations or photodissociation regions that are typically found in photoionized nebulae.

influence of dust to the temperature of the nebulae. A typical photoionized nebula will be characterized by a central source, surrounded by an $\mathrm{H}$ II region (or region of $\mathrm{H}^{+}$), which is, in turn, separated by a thin transition region from an outer neutral gas cloud or $\mathrm{H}$ I region. This is shown in Figure 1.2 , with the indication of some common ions produced in each region.

We will see, in Section 1.2, that the line ratios of forbidden collisionally-excited lines with respect to the hydrogen recombination lines are very useful to characterize gaseous nebulae, as these ratios depend on the ionic abundance and on the balance between excitation by electron collisions and de-excitation by both electron collisions and radiation (Pagel, 2009). There is a critical density where the collisional de-excitation probability equals the radiative de-excitation probability for the excited state of a determined element ${ }^{2}$ (Kewley et al. 2019). Above such critical density, the relative population of ions in the excited state will cease to increase with increasing electron density, and the forbid-

\footnotetext{
${ }^{2}$ The critical densities for collisional de-excitation of [O I] $\lambda 6300$, [O III] $\lambda 5007$, [S II] $\lambda \lambda 6716,6731$, and [N II] $\lambda 6583$ are $2 \times 10^{6}, 7 \times 10^{5}, 2 \times 10^{3}, 4 \times 10^{3}$, and $9 \times 10^{4} \mathrm{~cm}^{-3}$, respectively Osterbrock and Ferland, 2006)
} 
den line production (associated with radiative de-excitation) is suppressed since collisional de-excitation is no longer negligible (Osterbrock and Ferland, 2006).

The spectra of photoionized gaseous nebula present continuum emission produced by atomic processes associated to AGN and/or stellar emission or by scattering of light from the photospheres of stars within the nebula, and they also present absorption lines produced by stellar atmospheres or by ions and atoms of the ISM. However, in this thesis we will focus on emission lines, that are much harder to produce in typical astronomical environments. The most characteristic emission lines produced by gaseous nebulae are the forbidden lines of ions of common elements, such as the doublets [O III] $\lambda \lambda 4959,5007$, [N II] $\lambda \lambda 6548,6583$ and [S II] $\lambda \lambda 9069,9523$, and [O II] $\lambda \lambda 3726,3729$. The permitted lines of hydrogen, $\mathrm{H} \alpha \lambda 6563$ in the red, $\mathrm{H} \beta 4861$ in the blue, $\mathrm{H} \gamma \lambda 4340$ in the violet, and so on, are also characteristic of every optical nebular spectrum. The helium permitted lines are considerably weaker, with He I $\lambda 5876$ and He II $\lambda 4686$, the last being observed only in higher-ionization nebulae (Osterbrock and Ferland, 2006). These lines can provide information on, for example, the ISM density ([S II] or [O II] doublets), the ionization parameter (by the comparison of lines of the same element with different ionization levels, such as $[\mathrm{O} I],[\mathrm{O} \mathrm{II}]$, and $[\mathrm{O} \mathrm{III}]$ ), and the temperature (by [O III] or other calibrations with strong lines, described in Section 3.3.2) (Osterbrock and Ferland, 2006; Kewley et al., 2019).

Despite the similarities between the gaseous nebulae photoionized by stars and AGNs, there are also important differences, that are discussed in more detail in Section 3.3.2. The particle and energy densities are much larger in AGNs compared with star-forming regions, due to the harder radiation field of the former (Osterbrock and Ferland, 2006). The ionization by a power-law continuum also leads to a greater range in the electron temperature and ionization (Pagel, 2009), and some coronal emission lines are present in AGNs but not in H II regions. Hence, the nebulae around AGNs exhibit a more complex ionization structure in comparison to star-forming regions (Dors et al., 2020). In the following Sections, we will provide more details on the characteristics of black hole and stellar activities.

\subsubsection{Active galactic nuclei}

AGNs are the direct manifestations of a SMBH accreting matter in the center of galaxies (Lynden-Bell, 1969; Ho, 2008; Event Horizon Telescope Collaboration, 2019, see Figure 1.3 


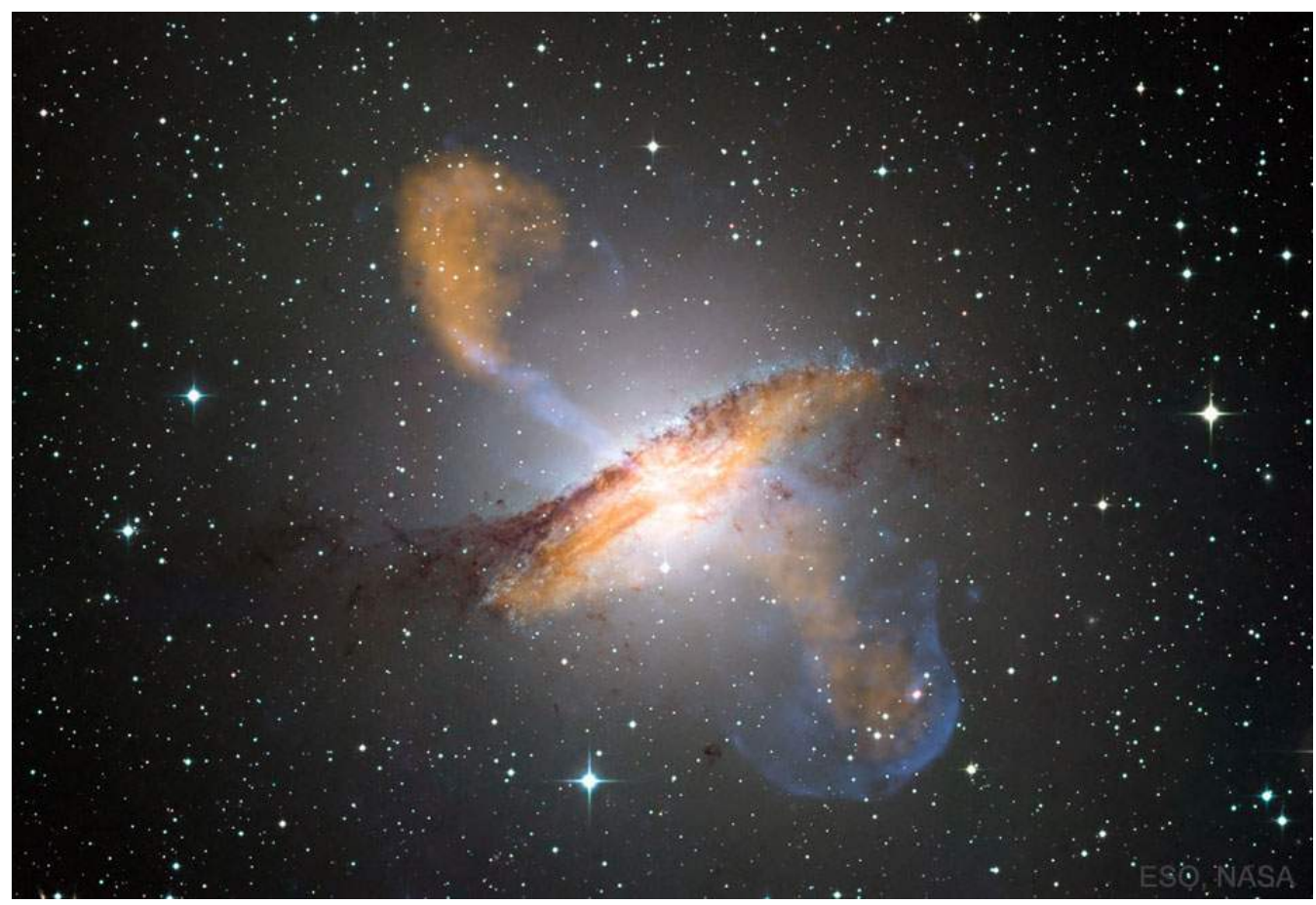

Figure 1.3: Galaxy Centaurus A and its jets. Image credit: ESO/WFI (visible); MPIfR/ESO/APEX/A. Weiss et al. (microwave); NASA/CXC/CfA/R. Kraft et al. (X-ray).

for an example). The spectral energy distribution (SED) of an AGN has both thermal and non thermal emission contributions, and it can be separated in several components such as radio synchrotron emission from jets (strong in radio-loud cases and weak in radio-quiet cases), an infrared (IR) excess (predominantly thermal radiation from a clumpy, dusty torus), a prominent optical to UV excess, the so-called "Big Blue Bump" (BBB, interpreted as pseudoblackbody emission from an optically thick, geometrically thin accretion disk), a soft X-ray excess (of controversial origin, e.g., Done et al. 2007; Miniutti et al.|2009), and an underlying power-law (most conspicuous at hard X-ray energies but thought to extend to IR wavelengths, associated with Comptonization of softer seed photons) (Ho, 2008). These characteristic spectra are found in many different types of objects, from early- to late-type host galaxies, including Seyferts, LINERs (Heckman, 1980), quasars, and radio galaxies (Osterbrock and Ferland, 2006). Thus, AGNs can be detected with a wide variety of techniques such as the detection of central hard X-ray point source, UV variability, broad emission lines (in direct or polarized light), and compact radio cores or extended radio jets/lobes (Ho, 2008; Yan and Blanton, 2012).

The hard extreme ultraviolet (EUV) radiation field is the main responsible for the high observed fluxes of collisionally-excited lines (Groves et al., 2004; Kewley et al., 2006, 2013). 
Many luminous AGNs such as quasars and Seyferts exhibit permitted emission lines with a characteristically broad component of full width at half maximum (FWHM) ranging from $\sim 1000$ to $10,000 \mathrm{~km} \mathrm{~s}^{-1}(\mathrm{Ho}, 2008)$. Such features are produced in the broad-line region (BLR), while the narrow-lines, corresponding to lower velocities, are produced in the narrow-line region (NLR) or even in the extended narrow-line region (ENLR) (e.g. Dopita et al., 2002; Groves et al., 2004, Ho, 2008; Congiu et al., 2017). The BLR is located closer to the central black hole and presents very high densities $\left(\sim 10^{10} \mathrm{~cm}^{-3}\right)$ and a large velocity spread $\left(\sim 3000 \mathrm{~km} \mathrm{~s}^{-1}\right)$, while the NLR is located further from the SMBH and is characterized by low gas density $\left(\sim 10^{4} \mathrm{~cm}^{-3}\right)$ and a much smaller velocity spread $(\sim 300$ $\mathrm{km} \mathrm{s}^{-1}$, Netzer 2013).

The unified model of AGNs (Antonucci, 1993; Netzer, 2015) shows how the orientation of the AGN, with respect to a dust-obscuring circumnuclear medium, affects the observed spectrum. This obscuring component is nowadays best described as a "clumpy" torus that does not fully cover the central source, causing some of radiation to escape and photoionize the gas of the NLR (Kauffmann et al., 2003). The type 1 AGNs refer to the cases in which the AGN and its BLR are observed directly, with narrow forbidden lines and broadened Balmer lines detected in the optical and UV bands. On the other hand, the type 2 AGNs are associated with AGNs viewed at an angle where light from the BLR is largely attenuated by the torus, while narrow forbidden emission and Balmer recombination lines from the NLR are still seen. In low-luminosity AGNs the BLR might not exist, and only NLR features are observed (Laor, 2003). Type 2 AGNs are more common than type 1 AGNs (Agostino and Salim, 2019), and the present work refers only to objects that do not present broad emission lines. Figure 1.4 presents a schematic representation of the unified model of AGNs.

Since the two necessary ingredients for creating a powerful AGN are a SMBH and an abundant fuel supply (Kauffmann et al., 2003), AGNs are no longer viewed as rare and exotic oddities, but rather as natural episodes during the life cycle of galaxies $(\mathrm{Ho}$, 2008). However, the accretion processes might be different depending on the amount of gas inside the gravitational potential of the black hole: if the supply of gas is plentiful, the SMBH regulates its own growth at a rate that does not further depend on the properties of the interstellar medium; once the gas runs out, the SMBH growth is regulated by the rate at which evolved stars lose their mass. There are other mechanisms that can 


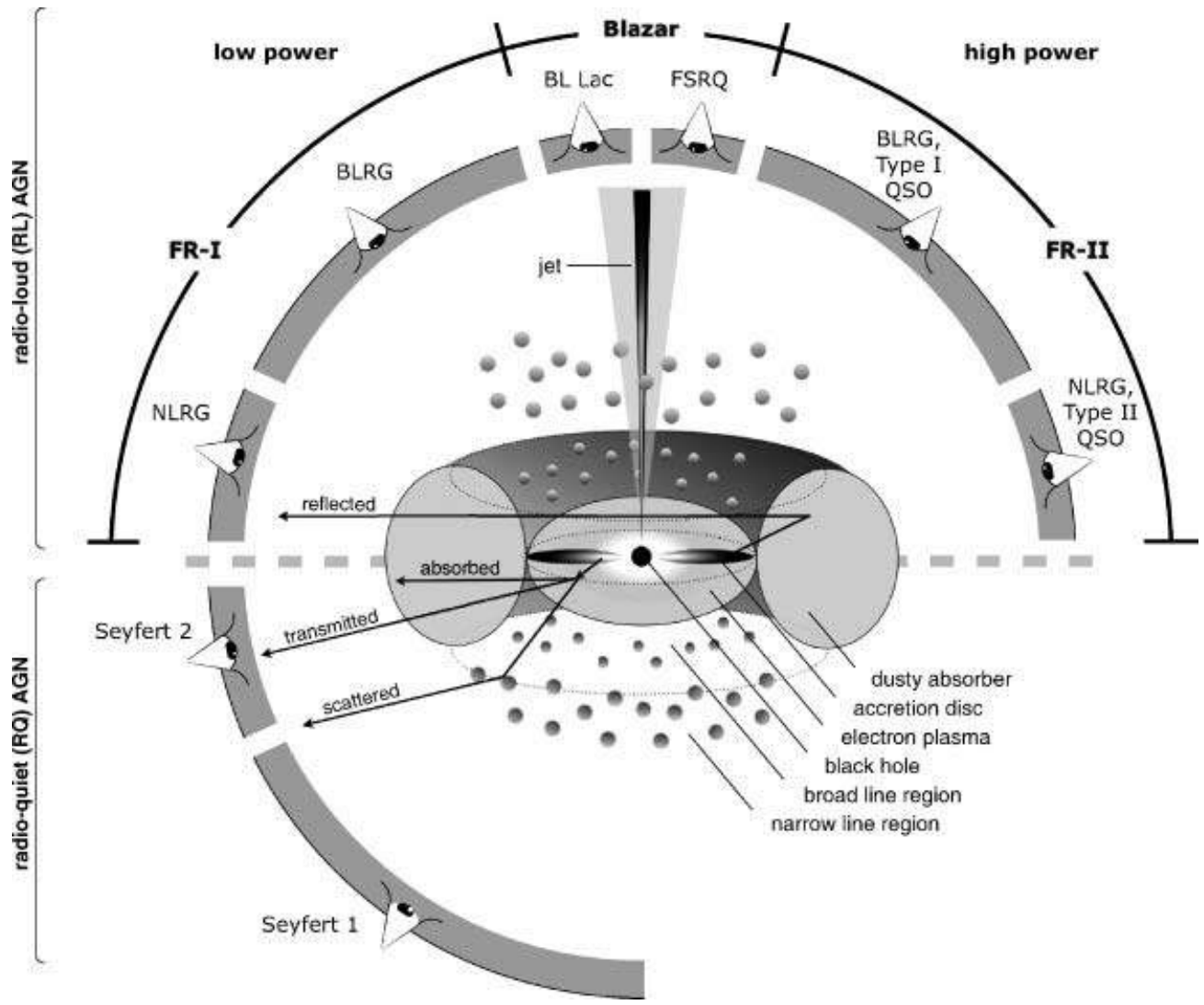

Figure 1.4: Figure 1.1 from Beckmann and Shrader (2012) (page 5) with the unified model of AGNs, showing a schematic representation of the understanding of the AGN phenomenon and its main components, in a simplified view that is not to scale.

drive gas into the nuclear region of galaxies, such as mergers and interactions, bar-driven instabilities, cooling flows, and cosmological inflows (see Kauffmann and Heckman, 2009. and references therein). Hence, though nearby AGNs reside almost exclusively in massive galaxies $\left(M \gtrsim 10^{10} \mathrm{M}_{\odot}\right.$, Kauffmann et al. 2003), there is a trend with morphological type of the host galaxy that is associated with the amount of gas available to fuel the central black hole. A standard evolutionary scenario, proposed by many authors, is the sequence in which star-forming galaxies from the blue cloud are eventually activated as AGNs. Their feedback suppresses star formation which moves the galaxy to the green valley of the colormass diagram, and eventually settles on the red sequence, when the gas supply is no longer able to sustain high accretion rates (Schawinski et al., 2007).

The unified model is an effort to associate the many different AGN properties with the same astronomical phenomenon observed under different inclinations. However, it is clear that orientation alone does not sufficiently explain the observed differences between the AGNs. Heckman and Best (2014) propose that the most relevant intrinsic parameter that 
differentiates the classes of objects is the accretion rate. Figure 1.5 shows the schematic representation of the so-called radiative-mode AGNs on the left and of the jet-mode AGNs on the right. The radiative-mode AGNs are associated with objects that have a BLR, either visible as in type 1 AGNs or obscured as in type 2. Seyferts, quasars (both radio loud and radio quiet) and high-excitation radio sources are all classified as radiative-mode AGNs. All these objects have an Eddington ratid ${ }^{3}$ above 1\%, indicating an efficient conversion of the potential energy of the gas accreted by the SMBH into electromagnetic radiation. They are also associated with a geometrically thin, optically thick accretion disk that reaches into the radius of the innermost stable orbit around the central black hole, and the luminous X-ray and UV radiation from such disk illuminates the BLR and NLR. There is an obscuring-structure of dusty molecular gas that can prohibit a direct view of the accretion disk and the BLR, as postulated by the unified model. Some radiativemode AGNs produce powerful radio jets as well, but this should not be confused by the second category of jet-mode AGN. The jet-mode AGNs are associated with LINERs and low-excitation radio sources presenting low accretion rates (Eddington ratios smaller than 1\%). These objects likely do not produce a thin accretion disk, and their inner regions may host a geometrically thick advection-dominated accretion flow (ADAF, often also called radiatively inefficient accretion flow, RIAF), with the transition to an outer truncated thin disk at larger distances from the central source. The jet-mode AGN are less powerful sources of ionization, but they can still ionize weak, low-ionization narrow-line regions. Their energy output is mainly in the form of kinetic energy through radio jets.

Hence, we have seen that, although Seyferts and LINERs can be distinguished by their ionization levels and luminosities (Heckman, 1980; Halpern and Steiner, 1983; Kauffmann et al. 2003; Ho, 2008), they are also intrinsically different, since the former is associated with high accretion rates and high efficiency in the conversion of gravitational energy into radiation, and the latter with low accretion rates and low efficiency (Kewley et al., 2006; Heckman and Best, 2014). Seyferts are usually found in spiral galaxies (Pagel, 2009), which are gas-rich environments that can fuel the nuclear activity. LINERs are more likely

\footnotetext{
3 The Eddington ratio is the ratio between the bolometric luminosity and the Eddington luminosity. The Eddington luminosity is an observable quantity that corresponds to the critical mass-accretion rate. Thus, the Eddington ratio is an estimate of the percentage of radiative luminosity that arises from accretion processes (Beckmann and Shrader, 2012).
} 
a Radiative-mode $A G N$

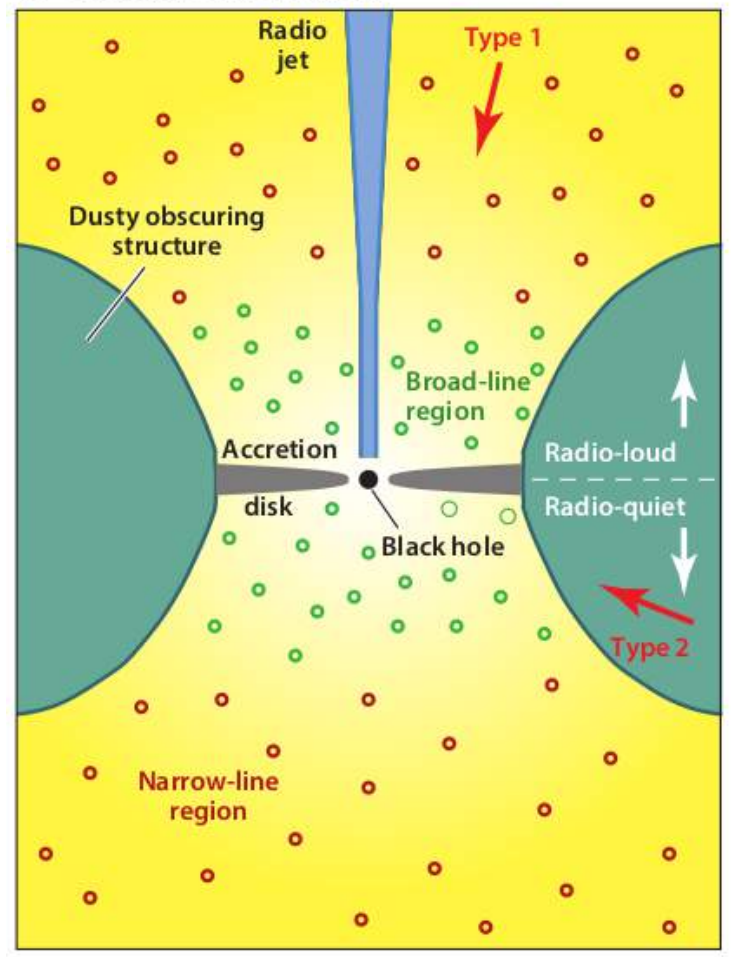

b Jet-mode AGN

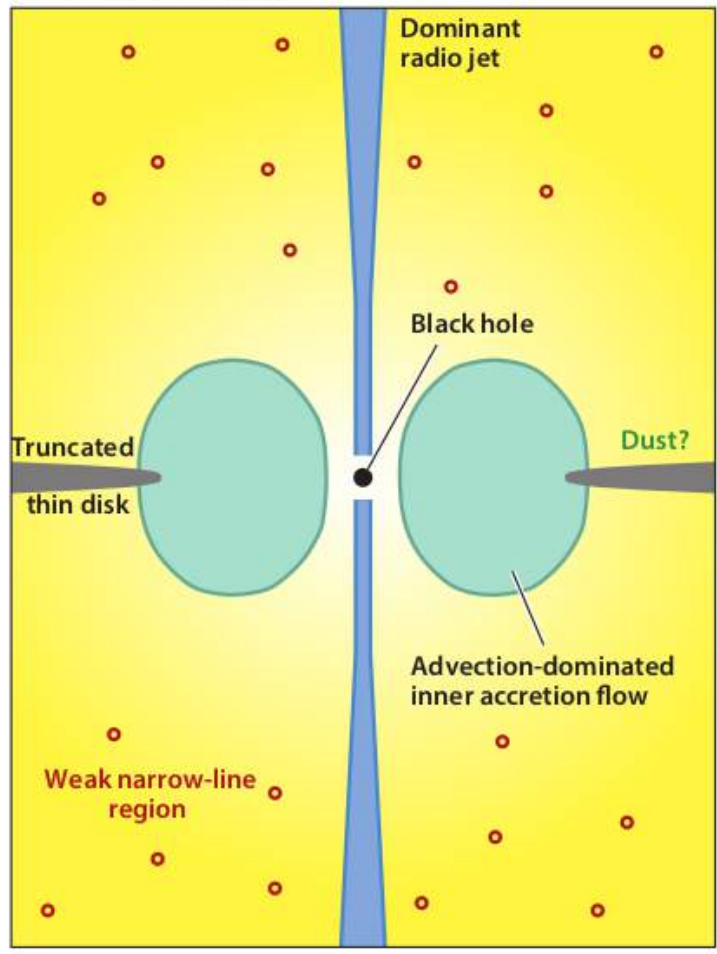

Figure 1.5: Figure 3 from Heckman and Best (2014), showing schematic drawings of the central engines of radiative-mode (left panel) and jet-mode (right panel) AGNs, not to scale.

found in massive early-type galaxies, which are more evolved environments with little gas (Kewley et al., 2006).

The LINER-like spectra are very similar to Seyfert-like spectra, but with stronger low ionization lines (Veilleux and Osterbrock, 1987; Kauffmann et al., 2003). They are extremely common, representing the majority of the nearby AGNs found in approximately one third of all galactic nuclei (Ho et al., 1997), especially in elliptical and early-type spiral host galaxies (Pagel, 2009). They were first defined as Low-Ionization Nuclear Emission-line Regions (LINERs) by Heckman (1980) as a class of AGNs with [O II] $\lambda 3727$ $\geq[\mathrm{O}$ III $] \lambda 5007$ and $3[\mathrm{O}$ I $] \lambda 6300 \geq[\mathrm{O}$ III $] \lambda 5007$ that represent the low-luminosity extension of the Seyfert population. Other notable features of the SED of low luminosity AGNs that coincide with the LINER-like spectra are the absence of a BBB, a shift of a broad excess to the mid-IR which is denominated "Big Red Bump", a steep optical to UV slope, no evidence of a soft X-ray excess and high values for the radio-to-optical luminosity ratios, indicating that they are usually radio-loud $(\mathrm{Ho}, 2008)$. However, though the majority of the LINERs are accretion-powered AGNs (Ferland and Netzer, 1983; Halpern and Steiner. 1983; Ho, 2008), other mechanisms are able to reproduce some of their characteristics, 
and the LINER class is more complex than simply assuming that all LINERs are AGNs (Stasińska, 1984; Dopita and Sutherland, 1995).

LINERs that are genuine AGNs (e.g., Ricci et al., 2014; da Silva et al., 2020) present properties that are consistent with a jet-mode scenario, where the energy released by viscous processes may be advected with the gas and consequently radiate inefficiently (ADAF for advection dominated accretion flow or RIAF for radiatively inefficient accretion flow). As a consequence of little radiative loss, the gas is extremely hot, and therefore LINERs present a lower-luminosity, but harder ionizing radiation field, with lower ionization parameter than Seyferts (Kewley et al., 2006).

However, some galaxies present LINER-like extended emission (i.e., $>1 \mathrm{kpc}$ ), not close to the central regions where the SMBHs reside (Phillips et al., 1986; Goudfrooij et al., 1994; Ricci et al., 2015). This extended LINER emission can be produced by shock waves (the shocks can be associated with stellar processes as mass loss, winds and supernovae, to AGN outflow, or to galactic scale outflows, Heckman 1980; Dopita and Sutherland 1995; Ho et al. 2014) or by hot evolved stars (Binette et al., 1994a; Stasińska et al., 2008; Sarzi et al. 2010; Cid Fernandes et al., 2011; Yan and Blanton, 2012)). However, the term LINER is not appropriate for such cases because they are not Nuclear, and such objects have been referred to as LIERs (e.g., Belfiore et al., 2016; Hsieh et al., 2017; Byler et al., 2019). Since shocks may be associated with AGN and bona fide LINERs are typically found in early-type galaxies (e.g., Heckman, 1980; Kauffmann et al., 2003; Kewley et al., 2006) that have older stellar populations, the three ionization mechanisms (stars, AGN and shocks) can act on the gas simultaneously in some cases (Kewley et al., 2019). Other important contributing sources are the Diffuse Ionized Gas (DIG, Collins and Rand 2001; Zhang et al. 2017; Vale Asari et al. 2019), cosmic ray heating, or cumulative X-ray emission from low-mass X-ray binaries (see $\mathrm{Ho}, 2008$, and references therein).

The contribution from multiple ionizing sources to a spectrum are extremely common and difficult to quantify, especially in the case of integrated spectra. Therefore, it is easier to classify galactic nuclei according to their dominant power source than to exactly describe how much each possible mechanism (AGNs, O and B stars, WR stars, hot low-mass evolved stars, shocks, cosmic rays, DIG, X-ray binaries, etc.) contributes to the overall resulting spectrum. Usually, strong AGNs also have young stellar populations in their host galaxies, that tend to be more gas- and dust-rich, while weak AGNs tend to reside in galaxies with 


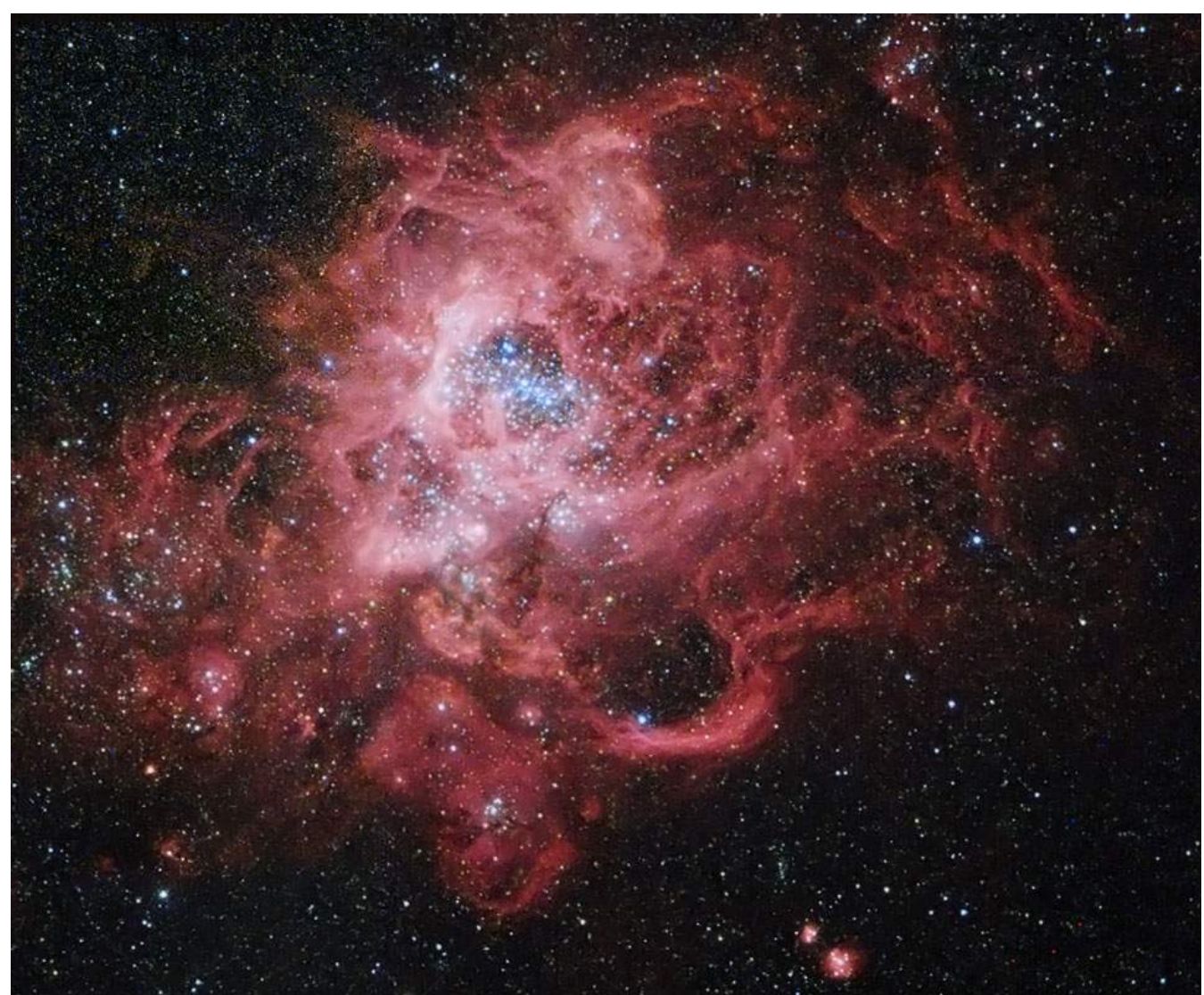

Figure 1.6: Image of NGC 604 (or M33), a region of ongoing star formation in the Milky Way. Image credit: Hubble Legacy Archive, ESA, NASA; Processing - Donald Waid.

older stellar populations and low gas and dust contents (Kauffmann et al., 2003). Hence, in the next Section we will provide a review of also the possible stellar related processes that ionize gaseous nebulae.

\subsubsection{Stellar activity}

Hot stars are able to photoionize the surrounding gas with their emission of ultraviolet radiation (Osterbrock and Ferland, 2006; Heckman, 2006). Such hot and massive stars might be young, associated with star formation in $\mathrm{H}$ II regions or starbursts, or they might be old, associated with evolved stellar populations. Another interesting stellar source that can make the ionizing spectra harder are the Wolf-Rayet stars. In this Section, we will detail some of the characteristics of these three types of photoionization. 


\subsubsection{Young $O B$ stars}

Many galaxies present characteristic nebular emission lines in the spectra of their nuclei that indicate that the gas is being photoionized by hot stars. These emission-line spectra provide constraints on the physical parameters of the gas being ionized and of the ISM in general, with information concerning the gas density, temperature, and pressure, and star-formation rate (Kewley et al., 2001). There might be a giant H in region (see Figure 1.6 for an example), or a cluster of several H II regions, or even a starburst event happening in the nuclei of galaxies (Osterbrock and Ferland, 2006). In order to consider all such cases, we will refer to $\mathrm{H}$ II region-like spectra as star-forming regions.

This photoionization profile is associated with newly born stars, that usually still reside in the gaseous nebula where they formed. Such young populations are associated with O and $\mathrm{B}$ stars, which are very massive $\left(M \gtrsim 2 \mathrm{M}_{\odot}\right)$, hot $(T \sim 20000-50000 \mathrm{~K}$, Heckman 2006), and, consequently, have short lifetimes on the main sequence (lifetime lower than 10 Myr, Leitherer et al. 1999). Once these stars are born, they immediately start to photoionize the residual ISM that surrounds them, thus creating a large emission-line diffuse nebulae, with observable features in the optical and UV ranges. The effective temperature of these early-type stars is a few times $10^{4} \mathrm{~K}$, and the emitted photons are able to ionize the nebula. The recombination of hydrogen or helium ions will result in emission lines by their resulting radiative cascades until the atoms reach the ground level. As for other elements (metals), the free thermal electrons in the gas will collisionally excite these ions and their radiative decay will produce emission lines (Heckman, 2006). These star-forming regions are also luminous in the IR range because of the dust, which absorbs the UV photons emitted by the hot stars and radiates the energy in IR photons (Osterbrock and Ferland, 2006).

These star-forming regions have typical densities of 10 to $10^{2} \mathrm{~cm}^{-3}$ in their ionized parts (e.g., Copetti et al., 2000), but higher density regions of $10^{4} \mathrm{~cm}^{-3}$ are also found (e.g., NGC 1976, Osterbrock and Ferland, 2006). The hot gas being ionized tends to expand into the surrounding neutral gas that is cooler, in such a way that the density within the nebula decreases while the ionized volume increases. Ionization fronts surround the outer edge of the nebula, running out into the neutral gas. Several structures can be formed in the distribution of the gas, such as bright rings, knots, and condensations Osterbrock and 
Ferland, 2006).

$\mathrm{H}$ II regions are associated with gaseous nebulae that are ionized by $\mathrm{O}$ and $\mathrm{B}$ stars, and they are found not only in the nuclei of galaxies, but also in the spiral arms of late-type galaxies as our own Milky Way (Baldwin et al., 1981; Osterbrock and Ferland, 2006). They contain mostly ionized hydrogen, presenting strong $\mathrm{H}$ and He recombination lines, but also strong collisionally excited lines such as [N II] and [O II], depending on the temperature of the ionizing stars (Osterbrock and Ferland, 2006).

The most luminous cases of galaxies with star forming regions, presenting the strongest emission lines (especially the hydrogen recombination lines relative to the continuum), are often called starburst galaxies (Weedman et al., 1981). These galaxies are undergoing an episode of intense star formation with much higher star-formation rate (SFR) than their past average $(\mathrm{Ho}, 2008)$, with values of the SFR ranging from $1-100 M_{\odot} \mathrm{yr}^{-1}$ (Heckman, 2006). The region of the galaxy where the starburst occurs is usually in the center, and the massive stars dominate the total luminosity of the entire galaxy. The starburst is fueled by a supply of interstellar gas that is primarily in the form of molecular hydrogen, and often associated with mergers and interactions (Osterbrock and Ferland, 2006).

Star-forming galaxies present a sequence on the plane of SFR and stellar mass, which is referred to as star-formation main sequence (Brinchmann et al., 2004). The origin of this sequence is often associated with the smooth mode of star formation that is common in galaxies with a continuous accretion of gas (Noeske et al., 2007). This sequence will be more evident in the diagnostic diagrams that will be presented in Section 3 .

\subsubsection{Wolf-Rayet}

Wolf-Rayet stars (WR) are hot stars with high luminosity and high mass-loss rates. These stars are the last stages of the evolution of massive stars, and the rise in their surface temperature leads to the removal of the outer hydrogen-rich layers via stellar winds, in such a way that their core with more advanced nuclear burning stages is exposed, leading to an increased detection of heavier elements (Lamers et al., 1991). Therefore, the spectra of WR are more peculiar than hot stars from the main sequence, with broad emission lines of helium, carbon, nitrogen, oxygen, and silicon, and with hydrogen weak or absent (Pagel 2009). The strong emission lines produced by their extended atmospheres (Leitherer et al. 1999) can sometimes mimic the power-law continuum of AGNs ( $\mathrm{Ho}, 2008)$, with a radiation 
field that is hard enough to generate high-ionization lines that a typical $\mathrm{H}$ II region is not able to reproduce (Dopita et al., 2013).

They are more abundant in metal-rich environments, as a result of the mass-loss rate dependency on metallicity (see D'Agostino et al., 2019, and references therein) and where a smaller mass is required for stars to reach the WR stage (Kewley et al., 2019).

\subsubsection{Hot Low-Mass Evolved Stars}

The last relevant source of stellar photoionization to be mentioned in this work is associated with hot low-mass evolved stars (HOLMES, Flores-Fajardo et al. 2011). This classification considers all the stars that are in the transition to become a white dwarf and are hot enough to ionize the surrounding medium (see the blue-shaded area in Figure 1.7). These could be pre-planetary nebulae (PPN), central stars of planetary nebulae (CSPN), hot pre-white dwarfs whose evolution time-scale is larger than that of the nebular envelope they ejected in a previous stage, post-asymptotic giant branch stars (pAGBs), post-early asymptotic giant branch stars (post-early AGB), and asymptotic giant branch-manqué (AGB-manqué), which are the result of insufficient envelope masses to allow a full AGB phase. Most of the HOLMES are not surrounded anymore by a planetary nebula, in such a way that the total number of ionizing photons that they produce is greater than what planetary nebulae alone could emit (Flores-Fajardo et al., 2011).

The radiation field produced by such old stellar population is much harder than those from young stars (Stasińska et al., 2008; Flores-Fajardo et al., 2011). We explain in more details the pAGBs since many authors have considered them as the main old stellar population to ionize the ISM, but HOLMES involve also other types of stars that have gone through similar processes with different details associated mainly to the initial mass, the life-time in the main sequence, and the envelope ejection.

The hot pAGBs are common in early-type galaxies, and along with white dwarfs, they are capable of producing a LINER-like spectra, being the main ionizing photon background in many galaxies that have ceased their star formation (Binette et al. 1994a). These LINER-like objects that are not a low-luminosity AGN are referred to as retired galaxies (Stasińska et al., 2008; Cid Fernandes et al., 2011). According to the Palomar Survey, about one third of the nebular emission in nearby galactic nuclei may be powered by photoionization from pAGBs $(\mathrm{Ho}, 2008)$. 


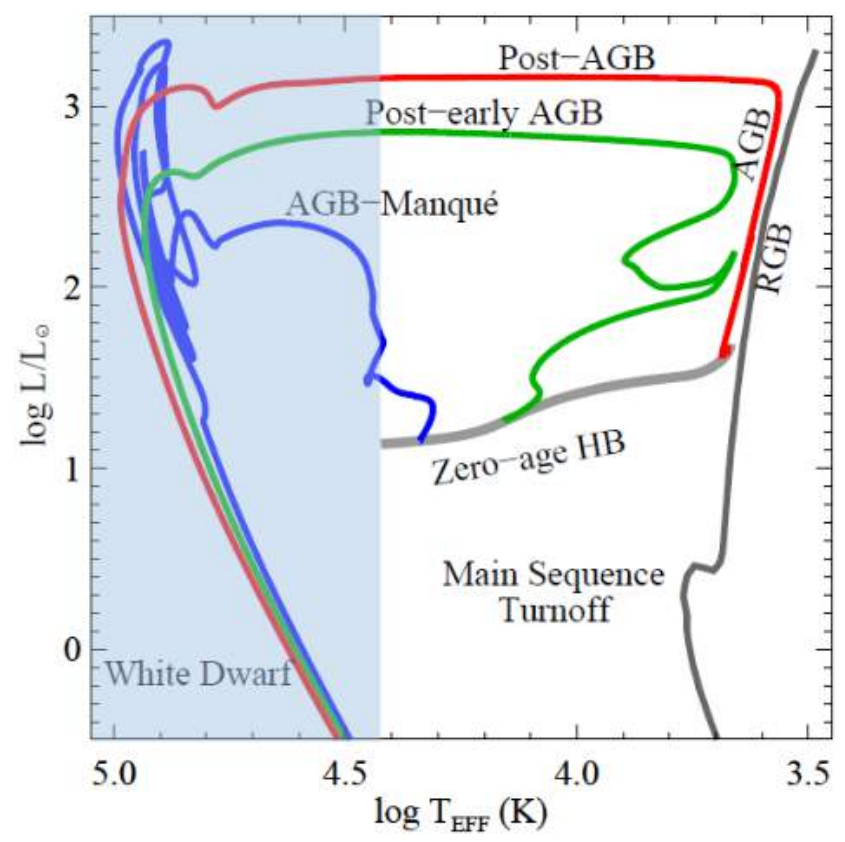

Figure 1.7: Fig. 1 from Brown et al. (2000) adapted by Paola Marigo. Stellar evolutionary tracks in the Hertzsprung-Russell diagram (luminosity versus temperature), with the blue-shaded area indicating the stars that can be considered HOLMES for being hot enough to ionize the medium. Such stars might come from different tracks (Post-AGB, Post-early AGB or AGB-manqué) and are evolving to become a white dwarf.

The pAGBs have left the asymptotic giant branch and are evolving horizontally on the Hertzsprung-Russell diagram, going towards very high temperatures $\left(\sim 10^{5} \mathrm{~K}\right)$ before they cool down to form white dwarfs (see Figure 1.7). They contain a degenerate core surrounded by a shell where they are burning hydrogen or helium. Since their temperatures are so high that they can ionize the medium around them and a lot of material has already been expelled from the stars in earlier evolutionary stages, the pAGBs are often accompanied by a planetary nebula, though this planetary nebula may disperse into the ISM while the pAGBs continue to produce ionizing radiation (Yan and Blanton, 2012).

\subsection{Diagnostic diagrams}

To better organize our understanding of the phenomena that occur in the nuclei of galaxies, it is interesting to develop the taxonomy of this field, which means, in practice, to have reliable classification schemes based on objective criteria. Usually, the classification schemes are based on phenomenology. Considering galaxies as an example, we have classifications based on morphology (Hubble, 1936), on photometric colors (de Vaucouleurs, 1960), on spectral features (Morgan and Mayall, 1957), among others. 
In this work, we will focus on the spectral classification of objects with emission lines. The spectrum of a galaxy contains several information about their ongoing physical processes. The optical spectrum, for example, can provide details about the chemical abundance, the dust, the electron density of the gas phase, the age of the stellar population, the pressure of the ISM, and the rate of star formation of the observed region. Considering only the intensity and shape of the emission lines, one already has access to information related to the physical conditions and the chemical composition of the gas, as well as on the dust contents of emitting regions (Cid Fernandes et al., 2010). They also reveal the power and nature of the ionizing source, such as the presence of an actively feeding SMBH (with details about the accretion rate, the shape of the AGN radiation field, and the strength of that radiation field) or the presence of shocks from massive stellar winds or collisions due to mergers, jets, or other (with details about the shock velocity, the density of the gas in the shock, and the mechanical energy of the shock). Therefore, a single galaxy spectrum allows one to determine the object's dominant power source, star formation history, chemical history and the prevalence of galactic-scale winds (Kewley et al., 2019). Specific spectral features or combinations of features used to infer the properties of a galaxy, these are denominated diagnostics, and when displayed in the form of a graph, these are called diagnostic diagrams. In order to be useful, the diagnostics need to be calibrated based on empirical or theoretical models, or both.

For galactic nuclei in particular, the diagnostic diagrams considering emission line ratios are the classification schemes that are most used and there are several ways of combining emission lines to obtain useful information.

The choice of the lines to be combined is very relevant. There are several considerations to take into account when proposing emission-line diagnostic diagrams. The most obvious requirement is that the lines must have a great signal-to-noise ratio $(\mathrm{S} / \mathrm{N})$, in order to be measured for many objects with the lowest uncertainty as possible and to avoid misclassification due to the low $\mathrm{S} / \mathrm{N}$. The combination of lines proposed must provide useful physical insight into the nature of the emitting system, able to distinguish between the different possible classes or to determine the parameter one wishes to constrain. In order to diminish effects of reddening and errors from flux calibration, combinations of lines with proximity in wavelength minimizes the reddening corrections due to dust while maximising diagnostic power (Veilleux and Osterbrock, 1987). Also, lines that are at nearby wavelengths sim- 
plify the observation process. Another topic to be considered is the difficulty with blended lines, because those can increase the error of the measured flux. Last, it is convenient to avoid lines that present large uncertainties in the fluxes as a consequence of the theoretical models that are required to calibrate them (Groves et al. 2004).

Section 1.2.1 will provide in more detail a historical review of the standard diagnostic diagrams, the emission-line classification scheme that was proposed firstly by Baldwin, Phillips \& Terlevich (1981) and later revised by Veilleux and Osterbrock (1987). The three resulting diagrams are major tools for classifying and analysing galactic nuclei, based on optical spectroscopy of ionized gas. The traditional diagnostic diagrams, ([O III $] \lambda 5007 / \mathrm{H} \beta$ versus $[\mathrm{N}$ II $] \lambda 6584 / \mathrm{H} \alpha,[\mathrm{O}$ III $] \lambda 5007 / \mathrm{H} \beta$ versus $[\mathrm{S} \mathrm{II}] \lambda(6716+6731) / \mathrm{H} \alpha$, and $[\mathrm{O}$ III $] \lambda 5007 / \mathrm{H} \beta$ versus $[\mathrm{O}$ I $] \lambda 6300 / \mathrm{H} \alpha){ }^{4}$ can clearly distinguish between galaxies dominated by star-formation from galaxies containing an active galactic nucleus. Based on emission-line ratios with Balmer lines, whose flux is directly proportional to the ionizing radiation of the power source, there is no dependency on luminosity for these diagnostic diagrams (D'Agostino et al., 2019).

The main lines used in these traditional diagnostic diagrams to distinguish the galactic nuclei based on their main excitation source, star formation, Seyfert or LINER, will be introduced together with the data used by this thesis in Section 3.1.1 of the analysis. Section 1.2 .2 shows some other diagnostic diagrams proposed in the literature, in the infrared, optical and ultraviolet domains.

The main idea behind the traditional diagnostic diagrams is that the emission lines in galactic nuclei with ongoing star formation are powered by massive OB stars, and thus there is an upper limit on the ratios of the intensities of collisionally excited lines with regard to recombination Balmer lines. For Seyferts and LINERs, however, the power source produces much more energetic photons, and therefore the collisionally excited lines will be more intense and the line ratios will reach higher values. Kewley et al. (2001) has shown with theoretical models that star-forming galaxies occupy a limited region in the diagnostic diagrams, even after accounting for variation in the gas-phase abundances and gas ionization state. The hard ionizing radiation field such as that from the accretion

\footnotetext{
${ }^{4}$ From here on, the optical emission lines [O III] $\lambda 5007$, [N II] $\lambda 6584,[\mathrm{~S} \mathrm{II}] \lambda(6716+6731)$ and $[\mathrm{O} \mathrm{I}] \lambda 6300$ will be referred to as simply [O III], [N II], [S II] and [O I]. The Balmer lines $\mathrm{H} \alpha$ and $\mathrm{H} \beta$ refer to $6563 \AA$ and $4861 \AA$, respectively.
} 
disk surrounding a SMBH is required to drive the emission line ratios to other regions in the diagnostic diagram where AGNs reside. Also, the star-forming nuclei form a well defined sequence, associated with the metallicity range of these objects and the mean effective temperature of the ionizing stars and ionization parameter (McCall et al., 1985). The AGNs also lie along a branch in the diagnostic diagram, but the objects are more scattered in comparison to the star-forming nuclei. This indicates that a few parameters are sufficient, mainly, again, the metallicity, the ionization parameter and the hardness of the spectral energy distribution, which, in this case, is associated with a smooth featureless power-law or a broken power-law EUV ionizing spectrum (Dopita et al., 2002). These two branches are often referred to as the wings of a seagull, especially in the diagnostic diagram that considers nitrogen on the abscissa (Stasińska et al., 2006).

However, even though the traditional diagnostic diagrams are a powerful tool to determine the main ionization mechanism within a source, this method also presents its caveats. The major issue with the use of the traditional diagnostic diagrams is the ambiguity when comparing the same object in different sets of line ratios. When the object lies close to the borderlines of the classes, it can be classified as different types of galactic nuclei depending on the considered diagram. This problem is even worse when the calculated line ratios are presented without their error bars, making also the uncertainties in the classification problematic. This problem is often faced, since many of the catalogs with emission line flux measurements are available without their respective error bars (see Dors et al., 2019, for examples of such cases). The conflicts between the classifications obtained in the different traditional diagrams are part of our motivation for introducing various alternative diagnostic diagrams in this work.

\subsubsection{Historical review}

Before proposing alternative diagnostic diagrams, it is convenient to study the traditional diagnostic diagrams: the context in which they were proposed, the contributions they made to the field, their caveats, and the open questions that remain. In order to do so, this section will review the papers Baldwin, Phillips \& Terlevich (1981) that proposed the method and Veilleux and Osterbrock (1987) that modified and refined it to the three diagrams that are most widely used nowadays.

Section 1.2.1.1 introduces the seminal work by Baldwin, Phillips \& Terlevich (1981) and 
the diagram that will be referred to as BPT hereafter, which considers the emission line ratios of $[\mathrm{O} \mathrm{III}] / \mathrm{H} \beta$ and $[\mathrm{N} \mathrm{II}] / \mathrm{H} \alpha$ as its axes. In Section 1.2.1.2, Veilleux and Osterbrock (1987) will be presented. The diagnostic diagrams that will be referred to as VO-S and VO-O were first introduced in this paper, maintaining $[\mathrm{O} \mathrm{III}] / \mathrm{H} \beta$ as the ordinate axis but the line ratios $[\mathrm{S} \mathrm{II}] / \mathrm{H} \alpha$ and $[\mathrm{O} \mathrm{I}] / \mathrm{H} \alpha$ as the abscissas, respectively.

The key idea behind the diagnostic diagrams based on strong optical emission line ratios is the discrimination of objects according to their dominant power source with regard to ionization and excitation (D'Agostino et al., 2019). The main physical difference that distinguishes narrow-line AGNs from star-forming nuclei is the photoionizing continuum: while star-forming regions are photoionized by UV photons from the thermal emission of hot OB stars, the activity associated with black hole growth produces a nonthermal powerlaw continuum that extends to X-rays (Veilleux and Osterbrock, 1987; Meléndez et al. 2014). The hardness of the spectra is the feature mostly responsible for distinguishing galactic nuclei with gas photoionized by star formation from those photoionized by AGNs, in a way that these classes occupy distinct loci in the diagrams.

The relative intensities of certain prominent emission lines can be used to probe the conditions of a gaseous nebula, as explained by $\mathrm{Ho}(2008)$. It is important to understand where the optical forbidden lines considered by the diagnostic diagrams are being emitted. The oxygen line [O I] arises from collisional excitation of the neutral oxygen by hot electrons, and its ionization potential of $13.6 \mathrm{eV}$ is practically identical to that of hydrogen, so, in an ionization-bounded nebula, [O I] will be preferentially produced in the partially ionized zone, which is a region where both neutral oxygen and free electrons coexist (VillarMartín et al., 1996). The conditions of this region are also favorable to sulfur and nitrogen once ionized, $\mathrm{N}^{+}$and $\mathrm{S}^{+}$, which have ionization potentials corresponding to $22.3 \mathrm{eV}$ and $29.6 \mathrm{eV}$, respectively. These two ions are also found in other regions of the nebula. Thus, normally, if $[\mathrm{N} \mathrm{II}]$ and $[\mathrm{S} \mathrm{II}]$ have strong intensities when compared to, for example, $\mathrm{H} \alpha$, [O I] will also be strong (this line is usually weaker than the other two). In H II regions and starburst nuclei, where the nebula is photoionized by massive stars, the partially ionized zone is very thin since the ionizing spectrum of $\mathrm{O}$ and $\mathrm{B}$ stars contains few photons with energies above $13.6 \mathrm{eV}$. Therefore, considering the optical spectra of star forming regions, the low-ionization transitions [N II], [S II], and especially [O I] are very weak. Conversely, a harder radiation field, such as the one of an AGN power-law continuum that extends into 
EUV and X-rays, can penetrate much deeper into an optically thick cloud, producing an extensive partially ionized zone and, in consequence, strong low-ionization forbidden lines. A hard AGN radiation field can also boost the emission of collisionally excited forbidden lines, since the temperature will be enhanced by its high thermal energy deposition rate (Ho, 2008).

As for the Balmer recombination lines of hydrogen, their intensity will be proportional to the ionizing photon flux, assuming that all ionizing photons are absorbed by the gaseous component (case $\mathrm{B}$, Osterbrock and Ferland, 2006). The choice of using $\mathrm{H} \beta$, a line that is weaker than $\mathrm{H} \alpha$, comes from a strategy based on wavelengths, as will be explained in the next Sections.

\subsubsection{Baldwin, Phillips \& Terlevich 1981}

The aim of the work by Baldwin, Phillips \& Terlevich (1981) is to investigate the merits of several emission-line intensity ratios in order to classify spectra of extragalactic sources. They consider combinations of emission lines that are easy to measure and show empirically that two dimensional diagrams with line ratios efficiently separate objects into normal H II regions (photoionized by OB stars), planetary nebulae, objects photoionized by a power-law continuum, and objects excited by shock-wave heating.

By the time the paper was written, the increase of emission-line galaxies data was already noticeable, and the need for a quantitative and efficient classification system was becoming urgent. By then, the unified model of active nuclei had not yet been proposed and extragalactic objects that presented emission lines were classified in many categories (e.g., narrow-line radio galaxies, broad-line radio galaxies, active galaxies, Seyfert 1, Seyfert 2, QSO, N galaxies, etc.) and the classification schemes were strongly dependent on selection criteria, morphology, or line widths. Baldwin, Phillips \& Terlevich (1981) points out that this obscured the factor that could link many of such objects into a class and separate them from other objects that were intrinsically different, which is the excitation mechanism operating on the line-emitting gas. The predominant excitation mechanism in extragalactic objects is usually associated with photoionization by OB stars, photoionization by a powerlaw continuum source, or shock wave heating. Planetary nebulae, which are photoionized by stars that in most cases are much hotter than normal galactic stars, were considered in the work of Baldwin, Phillips \& Terlevich (1981), but often not in other papers, that 
already exclude objects with emission lines that reside inside the Milky Way and that are not typically associated to bright galactic nuclei.

Since the classes with distinct excitation mechanisms mentioned above present characteristically different spectra, Baldwin, Phillips \& Terlevich (1981) suggests that a classification system based on the relative strengths of emission lines could clearly distinguish between the four possibilities introduced. It points out that it is not easy to distinguish power-law ionization from shock-wave heating considering a one-dimensional classification, since these types of sources are usually recognized by the presence of lines of simultaneously high and low ionization. It also suggests that the most useful lines should be easily measured.

The emission lines considered in the diagrams proposed by Baldwin, Phillips \& Terlevich (1981), based on observational experience and model calculations of other works, are [O II] $\lambda 3727$, He II $\lambda 4686, \mathrm{H} \beta \lambda 4861,[\mathrm{O}$ III] $\lambda 5007,[\mathrm{O}$ I] $\lambda 6300, \mathrm{H} \alpha \lambda 6563$, and [N II] $\lambda 6584$. Other lines that had potential use like [Ne III] $\lambda 3869$, [O III] $\lambda 4363$, and $\mathrm{H} \gamma \lambda 4340$ were avoided due to difficulties in their measurements because of possible blends with other emission and absorption lines. [Ne v] $\lambda 3426$ was also considered, but since none of the $\mathrm{H}$ II regions of their sample exhibited such emission line, its detection would already indicate the presence of an AGN without the need of producing a diagram. The lines mentioned above were combined in ratios of their intensities, with the goal of empirically separating gas clouds excited by shock heating power-law photoionization and $\mathrm{H}$ II regions.

The ratio $\log ([\mathrm{O} \mathrm{II}] \lambda 3727 /[\mathrm{O}$ III $] \lambda 5007)$ is considered in many of the proposed diagrams because of the high intensity of both lines and its capability of providing a sequence from low-excitation H II regions to planetary nebulae, despite of the problems due to the reddening corrections, since there is a large wavelength separation of the lines. Except for this line ratio, Baldwin, Phillips \& Terlevich 1981) tries to choose combinations of emission lines that would be as close to each other in wavelength as possible, in order to minimize errors that arise from reddening, and that involve forbidden lines of different chemical elements, since those would be more abundance-sensitive than the ones that involve just one heavy element and the Balmer lines. These considerations led to the diagnostic diagram referred to as $\mathrm{BPT}$, with the axes $\log ([\mathrm{O} \mathrm{III}] / \mathrm{H} \beta)$ versus $\log ([\mathrm{N} \mathrm{II}] / \mathrm{H} \alpha)$. This combination is not very sensitive to reddening and can be measured without even the need of accurate flux calibration. More than just technically convenient, the diagram presents four distinct 


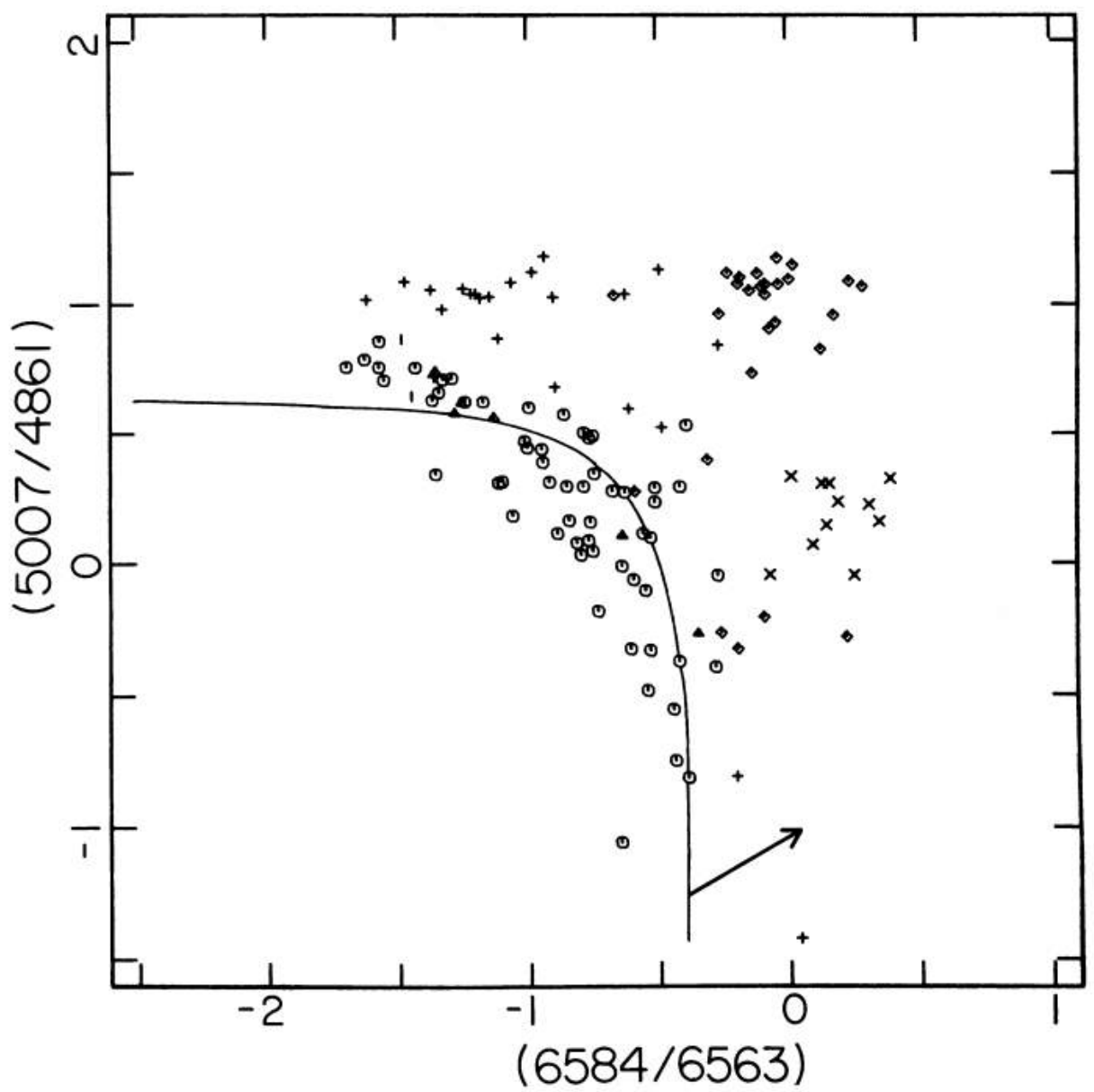

Figure 1.8: Figure 5 from Baldwin, Phillips \& Terlevich 1981), presenting the BPT diagnostic diagram. Octagons represent normal H II regions, triangles represent detached extragalactic H II regions, plus symbols represent planetary nebulae, diamonds represent objects photoionized by power laws, 'x' symbols represent shock-heated galaxies, and vertical bars represent the upper limits on $[\mathrm{O}$ III $] / \mathrm{H} \beta$.

regions corresponding to the four excitation mechanisms, as we can see in Figure 1.8, which is Figure 5 from Baldwin, Phillips \& Terlevich (1981). By considering the forbidden lines of nitrogen and oxygen, the BPT diagram is sensitive to the $\mathrm{N} / \mathrm{O}$ abundance ratio. Baldwin, Phillips \& Terlevich (1981) also points out the relevance of considering [O I] $/ \mathrm{H} \alpha$, which is a good indicator for the presence of a harder spectrum typical of power-law photoionization and/or shock-heating.

Therefore, the work from Baldwin, Phillips \& Terlevich (1981) presents several convenient quantitative classification schemes which consider the emission-line ratios of objects to separate them according to their primary excitation mechanism. The diagnostic diagram presented in Figure 5 from Baldwin, Phillips \& Terlevich (1981) (see Figure 1.8), with $\log ([\mathrm{O} \mathrm{III}] / \mathrm{H} \beta)$ versus $\log ([\mathrm{N} \mathrm{II}] / \mathrm{H} \alpha)$, is the main tool used to classify galactic nuclei and determine the ionization mechanism of the emission-line regions, distinguishing 
star formation from nuclear activity and also separating high- from low-ionization nuclear activity.

\subsubsection{Veilleux \& Osterbrock 1987}

The paper from Veilleux and Osterbrock (1987) proposes a revised method for the classification of narrow-line active galaxies and $\mathrm{H}$ II region-like galaxies that involves the line ratios $[\mathrm{O}$ III $] \lambda 5007 / \mathrm{H} \beta,[\mathrm{N} \mathrm{II}] \lambda 6583 / \mathrm{H} \alpha,[\mathrm{S} \mathrm{II}](\lambda 6716+\lambda 6731) / \mathrm{H} \alpha$, and $[\mathrm{O} \mathrm{I}] \lambda 6300 / \mathrm{H} \alpha$. The three proposed diagnostic diagrams are used to classify extragalactic objects, since these line ratios take advantage of the physical distinction between the two types of objects and also minimize the effects of reddening and errors in flux calibration.

Veilleux and Osterbrock (1987) considers a sample of previously classified emission-line galaxies (that includes Seyfert 2 galaxies, narrow-line radio galaxies, H II region galaxies and LINERs) with well-measured spectra to determine what are the main spectral features that can differentiate objects in which the photoionization is associated with a nonthermal power-law continuum or to hot $\mathrm{O}$ and $\mathrm{B}$ stars.

It was already known that the weakness of some low-ionization lines could distinguish star-forming regions from AGNs, as Baldwin et al. (1981) have shown for the case of [N II] and [O I] and as Keel (1983) has shown for the doublet of [S II]. As explained in Section 1.2.1 and also in Veilleux and Osterbrock (1987), the [S II] and [O I] emission lines arise preferentially in a zone of partially ionized hydrogen. This zone is extended in the case of nebulae photoionized by a spectrum with a large fraction of high-energy photons (as in the case of the hard radiation field produced by AGNs) and is nearly absent in nebulae photoionized by hot stars. This is the basis of the classification scheme that was proposed by Baldwin, Phillips \& Terlevich (1981). Therefore, Veilleux and Osterbrock (1987) proposes a classification method that takes full advantage of this by considering line ratios involving [O III], [N II], [S II], [O I] , and the Balmer lines, and excluding reddeningsensitive line ratios that were considered by Baldwin, Phillips \& Terlevich (1981) such as [O II] $\lambda 3727 /[\mathrm{O}$ III] $\lambda 5007$.

The five criteria behind the choice of the line ratios that maximize the diagnostic power of the classification schemes are the following: the lines must be strong and easily measurable in typical spectra; lines that blend with other lines should be avoided because that would increase the uncertainty in their flux measurements; the lines should be as 
close as possible with regard to wavelength so that their ratio is relatively insensitive to dust and flux calibrations; ratios involving a line of only one element and an $\mathrm{H}$ I Balmer line should be preferred to those involving forbidden lines of different elements, since the former are less abundance sensitive; the chosen lines should be in a wavelength region of the spectrum that is easily accessible with the contemporary instruments. Therefore, the emission-line ratios that satisfy all those criteria are [O III] $\lambda 5007 / \mathrm{H} \beta$ (which is proportional to $[\mathrm{O} \mathrm{III}](\lambda 4959+\lambda 5007) / \mathrm{H} \beta),[\mathrm{N} \mathrm{II}] \lambda 6583 / \mathrm{H} \alpha$ (which is proportional to $[\mathrm{N} \mathrm{II}](\lambda 6548$ $+\lambda 6583) / \mathrm{H} \alpha)$, and $[\mathrm{S} \mathrm{II}](\lambda 6716+\lambda 6731) / \mathrm{H} \alpha$. The blending of [N II] and $\mathrm{H} \alpha$ is troublesome but not too critical, since the available data usually have enough spectral resolution to determine their ratio with sufficient accuracy. The emission-line ratio of $[\mathrm{O} \mathrm{I}] \lambda 6300 / \mathrm{H} \alpha$ was also included, in spite of the weakness of [O I] line, because, according to these and several other authors, it is one of the best discriminators available between the objects photoionized by a power-law spectrum or by OB stars.

Veilleux and Osterbrock (1987) (Figure 1.9, which shows Figures 2 and 6 from the original paper) point out that the empty regions in the diagrams might be the consequence of selection effects, since galaxies with very low ionization tend to be weak in all their emission lines. That makes the subtraction of the stellar continuum even more important for measuring the weak emission lines in a low ionization galaxy reliably. It is argued that the bimodal distribution of AGNs, separating between Seyfert 2 nuclei and LINERs, is probably due to the absence of objects with intermediate $[\mathrm{O} \mathrm{III}] / \mathrm{H} \beta$, as it is less prominent than in Baldwin, Phillips \& Terlevich (1981). In spite of such caveats, the diagrams show a clear separation between H II region-like galaxies and narrow-line AGNs. Another important result is that $\mathrm{H}$ II regions and $\mathrm{H}$ II region-like galaxies occupy the same loci of the diagram.

Veilleux and Osterbrock (1987) also considers photoionization models, deriving the first semi-empirical classification lines to be used with the standard optical diagnostic diagrams and showing the shape that these classification schemes exhibit (Kewley et al., 2013, 2019). Considering photoionization by hot stars, they pointed out that the number of photons capable of ionizing $\mathrm{O}^{+}$will decrease if the ionization parameter and the stellar effective temperatures are decreased. As a consequence, the size of the zone where $\mathrm{O}^{++}$is found and the total flux of $[\mathrm{O}$ III] will also decrease.

With regard to nitrogen, for a fixed stellar temperature, as the ionization parameter 

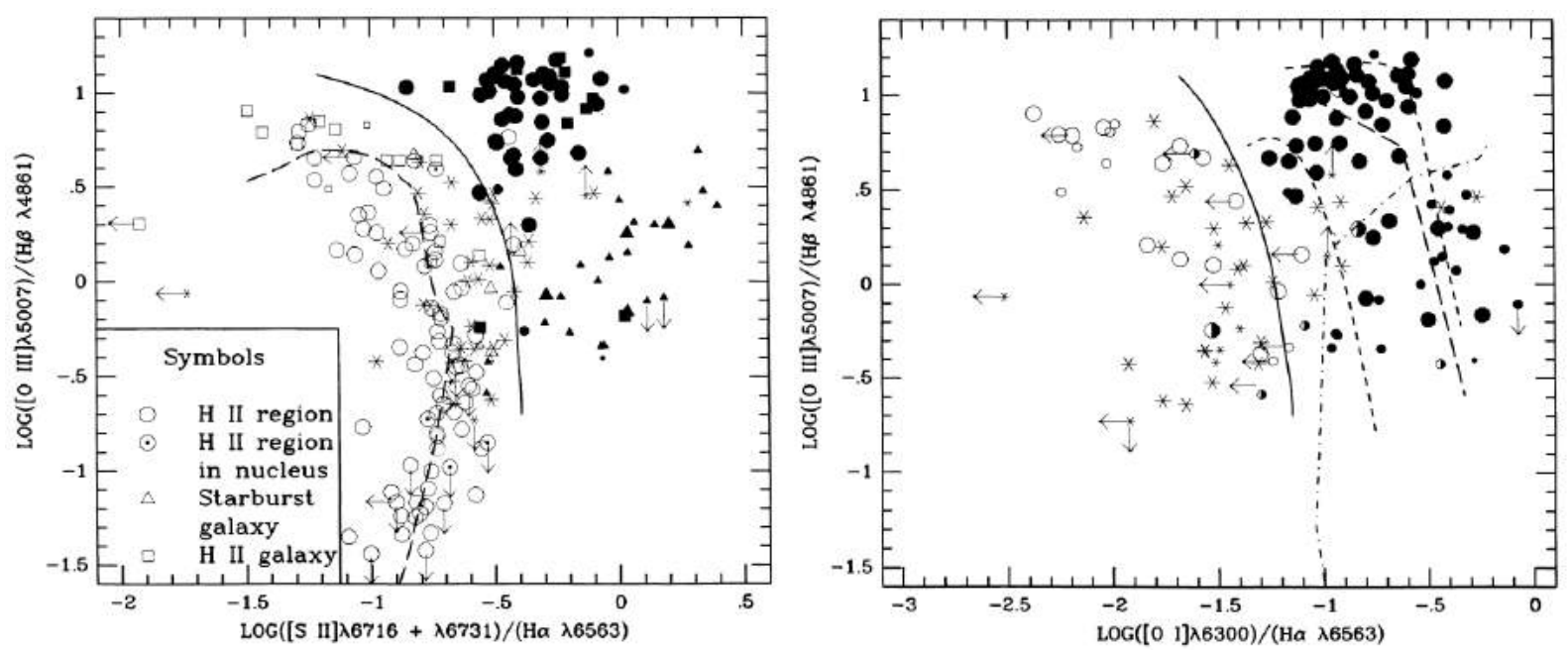

Figure 1.9: VO-S (left) and VO-O (right) diagrams from Veilleux and Osterbrock (1987). Filled circles represent Seyfert 2s, filled squares represent narrow-line radio galaxies (NLRGs), filled triangles represent LINERs, asterisks represent narrow-emission-line galaxies (NELGs). Open circles represent H II regions, dotted circles represent $\mathrm{H}$ II regions in nucleus, open triangles represent starburst galaxies, and open squares represent H II galaxies. Left: Figure 2 from Veilleux \& Osterbrock (1987), with models of star-formation. The dashed curve represents H in region models of McCall et al. (1985). The solid curve divides AGNs from H II region-like objects. Right: Figure 6 from Veilleux \& Osterbrock (1987), with models of AGN activity. Circles half filled are detailed in the original paper. The short-dashed curves represent power-law models of Ferland and Netzer (1983) for solar and 0.1 times solar abundances (upper and lower, respectively). Ionization parameter varies along curves from $10^{-1.5}$ to $10^{-4}$. The long dashed line represents a composite two-component (densities $10^{2}$ and $10^{6} \mathrm{~cm}^{-3}$ ) model of Stasińska (1984). Ionization parameter varies along curve from $10^{-2}$ to $10^{-4}$. The dot-dashed curve represents shock-wave models of Shull and McKee (1979). Shock velocity varies along the curve from less than 80 to $130 \mathrm{~km} \mathrm{~s}^{-1}$. The solid curve divides AGNs from H II region-like objects.

decreases, the zone that contains singly ionized species as $\mathrm{N}^{+}$will became steadily larger and can eventually fill the whole volume of the ionized $\mathrm{H}^{+}$zone. The same argument can be made for the partially ionized zone where [O I] is emitted. The models that present the hottest stellar temperatures will have the largest fraction of high-energy photons, and consequently the largest transition regions and more intense [O I] emission (among star forming regions).

As for the AGN, since their photoionizing continuum extends into the X-ray domain, photons with high energy will penetrate deeply into the predominantly neutral region, producing a large partially ionized zone, where $\mathrm{H}^{0}, \mathrm{H}$, and free electrons coexist with neutral atoms of other chemical elements and ions with an ionization potential similar to H. The prevalent forms of oxygen, sulfur, and nitrogen in this partially ionized zone are $\mathrm{O}^{0}, \mathrm{~S}^{+}$, and $\mathrm{N}^{0}$, with $\mathrm{N}^{+}$and $\mathrm{O}^{+}$also present. Hot free electrons produced in such region by X-ray photoionization can affect lines produced by collisional excitation, making them 
more intense. All the lines considered in the abscissas of the proposed diagnostic diagrams ([O I],$[\mathrm{S} \mathrm{II}]$ and $[\mathrm{N} \mathrm{II}]$ ) are of this type, and since this extended zone of partially ionized $\mathrm{H}$ does not exist in H II regions photoionized by hot stars (Osterbrock and Ferland, 2006), these lines will be relatively more intense in AGN than in star forming regions.

Considering the comparison between the observational line ratios of star-forming regions and narrow-line AGNs, Veilleux and Osterbrock (1987) explains the theory that justifies the different positions in the diagnostic diagrams. The emission lines [O I], [S II], and [N II] will be stronger with respect to $\mathrm{H} \alpha$ in AGNs than in $\mathrm{H}$ II region-like galaxies because the collisional excitation of such lines is more important in objects with extended partially ionized zones. This effect is more significant for $[\mathrm{O} \mathrm{I}] / \mathrm{H} \alpha$ because the ionization potential of $\mathrm{O}^{0}$ matches the ionization potential of $\mathrm{H}$ very well, while for $[\mathrm{S} \mathrm{II}]$, though this effect is still important, $\mathrm{S}^{+}$can also exist within the $\mathrm{H}^{+}$zones of both kinds of nebula, attenuating the distinction between the two classes. As for $[\mathrm{N} \mathrm{II}]$, since $\mathrm{N}^{+}$is present in the outer partially ionized zone but is not the predominant stage, one would expect it to be strengthened (though not as greatly as [S II]) in the absence of collisional de-excitation. However, the high discrepancy observed might be related to AGNs presenting higher nitrogen abundances or higher equilibrium temperature than $\mathrm{H}$ II regions. Concerning the ordinate axis, $\mathrm{O}^{++}$is produced by UV photons with energies above $35 \mathrm{eV}$ well inside the ionized zone close to the ionizing source. Nevertheless, the relatively large number of photons produced will generally make $[\mathrm{O} \mathrm{III}] / \mathrm{H} \beta$ more intense in these objects, with exceptions regarding the highest excitation star forming nuclei.

Finally, Veilleux and Osterbrock (1987) suggest that, when trying to decide whether a galactic nuclei is associated with an HII region or an AGN, more weight should be given to the diagram of $[\mathrm{O} \mathrm{I}] / \mathrm{H} \alpha$, since the separation between the two classes of objects is more distinct. They also notice that a small fraction of objects are in one class in certain diagram(s) and in another in other diagram(s). This missclassification issue will be discussed in Section 3.1.3.

Veilleux and Osterbrock (1987) has shown that the reddening-insensitive line ratios $[\mathrm{O} \mathrm{III}] / \mathrm{H} \beta,[\mathrm{N} \mathrm{II}] / \mathrm{H} \alpha,[\mathrm{S} \mathrm{II}] / \mathrm{H} \alpha$, and $[\mathrm{O} \mathrm{I}] / \mathrm{H} \alpha$ are very useful in classifying emission-line galaxies into narrow-line active galaxies and $\mathrm{H}$ II region galaxies. 


\subsubsection{Other wavelengths}

Other useful diagnostic diagrams have been proposed in the literature, though they are less widely used than the standard diagnostic diagrams of Baldwin, Phillips \& Terlevich (1981) and Veilleux and Osterbrock (1987).

First, we will mention the diagrams that can differentiate HOLMES from other ionizing sources. That is usually done by considering not only emission-line ratios, but also equivalent widths $(\mathrm{EW})$ of emission lines and/or the break at $4000 \AA\left(\mathrm{D}_{\mathrm{n}}(4000)\right)^{5}$. The $\mathrm{EW}(\mathrm{H} \alpha)$ distribution presents a dichotomy between star-forming galaxies and AGNs, despite significant overlap (Cid Fernandes et al., 2010). The $\mathrm{D}_{n}(4000)$ parameter increases monotonically as the mean age of the stellar population increases (Kauffmann et al., 2003).

The DEW diagram proposed by Stasińska et al. (2006) considers $D_{n}(4000)$ versus the ratio of the maximum $\operatorname{EW}([\mathrm{Ne} I \mathrm{II}] \lambda 3869,[\mathrm{O} \mathrm{II}] \lambda 3727)$, and it can be used with the optical spectra for galaxies up to $z=1.3$ (the limit in which the lines are redshifted and thus unavailable). The spectral features also have the advantage of not requiring stellar synthesis analysis to subtract the starlight, being more inclusive with regard to passive galaxies. Kobulnicky and Phillips (2003) show that emission lines EW can be used to estimate the global metallicities of galaxies, and this is the main reason for why starforming nuclei can be separated from AGNs in the DEW diagram. However, a possible drawback for this classification scheme is that old galaxies with a recent starburst might be mistaken for AGN hosts.

Another important diagnostic diagram that considers equivalent widths is the WHAN diagram proposed by Cid Fernandes et al. (2010, 2011). The classification schemes of the standard diagnostic diagrams (Stasińska et al., 2006, Kewley et al., 2006) are translated to this more economic approach, where the horizontal axis is the same as in the BPT diagram $(\log [\mathrm{NII}] / \mathrm{H} \alpha)$ but the ordinate axis is $\mathrm{EW}(\mathrm{H} \alpha)$. The $\mathrm{EW}(\mathrm{H} \alpha)$ indicates the power of the ionizing source with respect to the optical output of the host galaxy stellar population by measuring the amount of ionizing photons absorbed by the gas relative to the stellar mass. The $[\mathrm{N}$ II $] / \mathrm{H} \alpha$ line ratio, as already mentioned, depends on nitrogen abundance, the ionization state, and temperature of the gas. The WHAN diagram can separate not only

\footnotetext{
${ }^{5} D_{n}(4000)$ is the ratio between the average value of $F_{\nu}$ in the $4050-4250$ and $3750-3950 \AA$ bands (Bruzual, 1983), using the narrower bands 3850-3950 and 4000-4100 $\AA$ introduced by Balogh et al. 1999) to reduce reddening effects.
} 
star-forming galaxies, strong and weak AGNs, but also "retired galaxies" that have stopped forming stars and are ionized by old stellar populations. One of the main drawbacks of this classification scheme is its aperture dependence, with possible dilution from stellar sources in the circumnuclear regions. Cid Fernandes et al. (2010) also proposes diagrams where [O III] is maintained in the ordinate axis, but substituting $\mathrm{H} \beta$ as a denominator for stronger lines such as $\mathrm{H} \alpha$ or [O II], though these line ratios are more sensitive to reddening.

Among diagnostic diagrams, the approaches as Baldwin, Phillips \& Terlevich (1981), Veilleux and Osterbrock (1987), and Cid Fernandes et al. (2011) separate the objects according to their dominant ionizing source. Other diagrams, however, are focused on distinguishing objects with respect to metallicity, density, pressure, temperature and ionization parameter separately (see the review from Kewley et al., 2019).

In the optical domain 6 many combinations of emission-line ratios have been introduced, and we will briefly mention some of the most relevant works that proposed these alternative diagnostic diagrams. Some alternative line ratios were suggested by the work from Baldwin, Phillips \& Terlevich (1981), such as [O II] $\lambda \lambda 3727,9 /[\mathrm{O}$ III $] \lambda 5007$ and [O I] $\lambda 6300 /[\mathrm{O}$ III $] \lambda 5007$. Several emission-line ratios were proposed by Michael Dopita, and the ones ialguém mais tentou? alguma dificuldade que a gente possa ajudar?n the optical regime are $\mathrm{R}_{23}=([\mathrm{O}$ II $] \lambda \lambda 3727,9+[\mathrm{O}$ III $] \lambda \lambda 4959,5007) / \mathrm{H} \beta,[\mathrm{O}$ III $] \lambda \lambda 4959,5007 /[\mathrm{N}$ II $]$ $\lambda \lambda 6548,84$ (Dopita and Evans, 1986), [Nev] $\lambda 3426 /[\mathrm{Ne}$ III] $\lambda 3869$ (Evans and Dopita, 1986), [O II] $\lambda \lambda 7318,24 / \mathrm{H} \alpha,[\mathrm{S} \mathrm{III}] \lambda 9069,9531 / \mathrm{H} \alpha$ (Dopita and Sutherland, 1995), [O III] $\lambda 5007 /[\mathrm{O}$ II] $\lambda \lambda 3727,9$, [O II] $\lambda \lambda 3727,9 /[\mathrm{S} \mathrm{II}] \lambda \lambda 6717,31$ (Dopita et al., 2000), [O I] $\lambda 6300 /[\mathrm{N}$ iI] $\lambda 6584,[\mathrm{~N}$ I] $\lambda 5200 /[\mathrm{O}$ II] $\lambda \lambda 7318,24$ (Groves, Dopita \& Sutherland, 2004), [O III] $\lambda 5007 /[\mathrm{S}$ II] $\lambda \lambda 6717,31$, [S III] $\lambda 9069 /[\mathrm{S} \mathrm{II}] \lambda \lambda 6717,31$ (Dopita et al., 2013), among others. Dopita and Sutherland (1995) proposes the emission-line ratio of He II $\lambda 4686 / \mathrm{H} \beta$, which is also used by Binette et al. (1996) and Shirazi and Brinchmann (2012). Binette et al. (1996) also proposes the line ratios of [N $\mathrm{II}] \lambda 6584 /[\mathrm{O}$ II $] \lambda \lambda 3727,9,[\mathrm{O}$ II] $\lambda \lambda 3727,9 /[\mathrm{Ne} \mathrm{V}] \lambda 3426$, $[\mathrm{NeV}] \lambda 3426 / \mathrm{H} \beta$, and [O III] $\lambda 4363 /[\mathrm{O}$ III] $\lambda 5007$. Other relevant emission-line ratios are [Ne III $] \lambda \lambda 3869,3968 / \mathrm{H} \beta($ Rola et al. 1997$), \mathrm{S}_{23}=([\mathrm{S} \mathrm{II}] \lambda \lambda 6717,31+[\mathrm{S}$ III $] \lambda \lambda 9069,9531) / \mathrm{H} \beta$

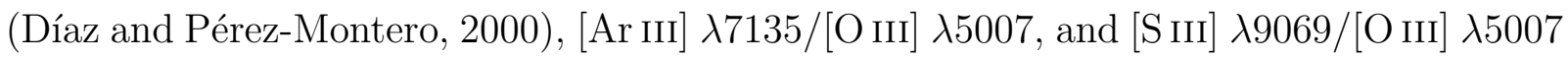

\footnotetext{
${ }^{6}$ We consider the optical domain referring to the wavelength range that can be obtained with typical groundbased optical instrumentation in the wavelength range from the atmospheric cutoff at $\sim 3300 \AA$ to $\sim 1 \mu \mathrm{m}$.
} 
(Stasińska, 2006). Several diagnostic diagrams combining the mentioned lines were proposed (see Nagao et al., 2006, Levesque et al., 2010; Dors et al., 2011; Dopita et al., 2013, and references therein).

With more recent techniques of integral field spectroscopy, diagnostic diagrams can be used to classify different regions according to the dominant ionizing source for each spaxel and combine the usual emission-line ratios with a kinematic information such as the velocity dispersion (D'Agostino et al., 2019).

Moreover, diagnostic diagrams have been proposed for wavelengths other than optical.

Ultraviolet diagnostic diagrams are useful to identify shocks, and also to classify galaxies at high redshift that will present such lines in the optical domain when observed with ground-based telescopes (Feltre et al., 2016, Inskip et al., 2002). Since shock heating usually achieves higher equilibrium electron temperatures than pure photoionization, highionization UV lines as He II $\lambda 1640,[\mathrm{~N}$ v] $\lambda 1240$ and $\mathrm{C}$ IV $\lambda \lambda 1548,51$ are advantageous $\mathrm{Ho}$ 2008). The line ratio C III] $\lambda \lambda 1906,9 /$ He II $\lambda 1640$ is very useful for separating star-forming regions from harder ionizing sources (Kewley et al., 2019). Other relevant UV emission lines that are often combined in line-ratios are C III $\lambda$ 977, C II] $\lambda 2326, \mathrm{~N}$ III $] \lambda 1750, \mathrm{~N}$ III $\lambda 991$ (Binette et al., 1996; Villar-Martín et al., 1996, 1997; Allen et al., 1998, 2008; Best et al., 2000; Moy and Rocca-Volmerange, 2002; Groves et al., 2004; Feltre et al., 2016 Matsuoka et al., 2018), C II $\lambda 1335$, S II $\lambda 1263$, S III $\lambda 1883$, O II $\lambda 2471$, O III $\lambda \lambda 1661,6$, O III $\lambda 2322$, and N III $\lambda 1752$ (Byler et al., 2018), for example. These lines are useful for estimating the temperature, the density, the metallicity, and the ionization parameter of the objects. The diagnostic diagrams with these emission lines can be used also to check the consistency of models that consider shocks, photoionization including dust, or matterversus ionization-bounded clouds (e.g., Binette et al., 1996; Allen et al., 1998; Groves et al. 2004 ; Feltre et al. 2016).

Diagnostic diagrams in the infrared are very important for the study of objects heavily obscured by dust, especially AGNs that would be classified as star-forming regions in the optical and UV diagrams (Kewley et al. 2019). The emission-lines in general have different critical densities and ionization potentials, in a way that precise characterization of the physical properties and excitation mechanisms can be revealed. Lowionization lines in the infrared such as [Ne II] $\lambda 12.8 \mu \mathrm{m}$, Fe II $\lambda 26.0 \mu \mathrm{m}$, and [S II] $\lambda 34.8 \mu \mathrm{m}$ are common in star-forming galaxies, while high-ionization lines such as [O IV] $\lambda 25.9 \mu \mathrm{m}$, 
$[\mathrm{NeV}] \lambda 14.3 \mu \mathrm{m} / \lambda 24.3 \mu \mathrm{m}$ and [NeIII] $\lambda 15.5 \mu \mathrm{m}$ are good indicators of AGN (Ho, 2008). Some diagrams can identify WR stars (Meléndez et al., 2014). Hence, some of the main infrared emission-line ratios used for diagnostic diagrams are [Ne III $] \lambda 15.5 \mu \mathrm{m} /[\mathrm{Ne} \mathrm{II}]$ $\lambda 12.8 \mu \mathrm{m},[\mathrm{Ne}$ IV] $\lambda \lambda 14.3,24.3 \mu \mathrm{m} /[\mathrm{Ne}$ II] $\lambda 12.8 \mu \mathrm{m},[\mathrm{O}$ IV $] \lambda 25.9 \mu \mathrm{m} /[\mathrm{Ne}$ II] $\lambda 12.8 \mu \mathrm{m},[\mathrm{O}$ IV] $\lambda 25.9 \mu \mathrm{m} /[\mathrm{O}$ III $] \lambda 88.3 \mu \mathrm{m},[\mathrm{O}$ III $] \lambda 51.8 \mu \mathrm{m} /[\mathrm{O}$ III $] \lambda 88.3 \mu \mathrm{m}$, and [S IV] $\lambda 10.5 \mu \mathrm{m} /[\mathrm{S} \mathrm{III}] \lambda 18.7 \mu \mathrm{m}$. Other relevant line ratios are Ar II $\lambda 6.98 \mu \mathrm{m}$, Ar III $\lambda 8.99 \mu \mathrm{m},[\mathrm{O}$ I] $\lambda 63 \mu \mathrm{m}$, [O I] $\lambda 14.5 \mu \mathrm{m}$, O III $\lambda 51.8 \mu \mathrm{m},[\mathrm{N} \mathrm{II}] \lambda 121.7 \mu \mathrm{m},[\mathrm{N} \mathrm{II}] \lambda 205.4 \mu \mathrm{m},[\mathrm{N} \mathrm{III}] \lambda 57.3 \mu \mathrm{m},[\mathrm{Fe} \mathrm{II}] \lambda 25.9 \mu \mathrm{m},[\mathrm{C}$ II] $\lambda 158 \mu \mathrm{m}$ (e.g., Voit, 1992; Spinoglio and Malkan, 1992; Groves et al., 2004; Snijders et al., 2007; Allen et al., 2008; Hao et al., 2009; Weaver et al., 2010; Nagao et al., 2011; Dopita et al., 2013; Fernández-Ontiveros et al., 2016). Some other approaches consider [Fe II] $\lambda 1.644 \mu \mathrm{m} / \mathrm{Br} \gamma$ or [Fe II] $\lambda 1.2567 \mu \mathrm{m} / \mathrm{Pa} \beta$ (Alonso-Herrero et al., 1997), or the IR continuum polycyclic aromatic hydrocarbon (PAH) features (e.g., Laurent et al., 2000; Spoon et al., 2007; Armus et al., 2007).

As for diagnostic diagrams for galaxies at higher redshift, it is important to consider that such objects trace earlier evolutionary phases of the Universe. Galactic nuclei at higher redshift will usually present higher densities, lower metallicities, and harder radiation fields (e.g., Strom et al., 2017, 2018). Therefore, they will occupy different positions on the diagnostic diagrams than local galaxies, and other classification schemes have been proposed (e.g., Kewley et al., 2013).

\subsection{Motivation}

The main goal of this thesis is to propose alternative diagnostic diagrams and classification schemes to classify galactic nuclei according to their main ionization source. To do so, we have revised the standard diagnostic diagrams (Baldwin et al., 1981; Veilleux and Osterbrock, 1987) and the main spectral classification schemes proposed (Ho et al., 1997; Kewley et al., 2001; Kauffmann et al., 2003; Kewley et al., 2006; Stasińska et al., 2006; Schawinski et al., 2007; Cid Fernandes et al., 2010; Meléndez et al., 2014).

The alternative diagnostic diagrams use the same emission-line ratios as their axes, in linear combinations $(\log [\mathrm{O} \mathrm{III}] / \mathrm{H} \beta \pm \log [\mathrm{O} \mathrm{I}] / \mathrm{H} \alpha$ versus $\log [\mathrm{N} \mathrm{II}] / \mathrm{H} \alpha \pm \log [\mathrm{S} \mathrm{II}] / \mathrm{H} \alpha$ and $\log [\mathrm{N} \mathrm{II}] / \mathrm{H} \alpha$ versus $\log [\mathrm{S} \mathrm{II}] / \mathrm{H} \alpha$ ), combining information that the three classical diagrams provide in a single graph while reducing issues of ambiguity and missclassification. We 
have chosen spectra with high $\mathrm{S} / \mathrm{N}$ to compose our sample in order to have more reliable classification schemes.

In order to examine how each parameter affects the position of an object in the alternative diagnostic diagrams being proposed, we have run theoretical models (using STARBurst99, Leitherer et al. 1999, and Cloudy, Ferland et al. 2017) that should reproduce the observed values of the emission lines. Therefore, is possible to better constrain the influence of physical parameters such as metallicity, ionization parameter, density, spectral index of the power-law radiation field, and age of the stellar population in altering the position of the models in the diagnostic diagrams. We can also notice that the simplistic single density models are not complex enough to properly describe the observed data.

Therefore, in this thesis we present a review of the diagnostic diagrams, with the classical classification schemes applied to our sample, and propose alternative diagnostic diagrams with new classification schemes based on emission-line ratios in the optical domain. We also propose a relation for the nitrogen abundance in star-forming regions, motivated by the photoionization models. 
Chapter 2

\section{Sample}

\subsection{Survey requirements}

When Veilleux and Osterbrock (1987) proposed the diagnostic diagrams that have been used ever since as the main tool to classify galactic nuclei in nearby objects, it was also suggested that the best way to test our understandings about the distinct mechanisms that are responsible for the ionization of the gas in emission-line galaxies would be to consider a large sample of these objects with well-measured spectra, thus requiring a $\mathrm{S} / \mathrm{N}$ criterion for obtaining accurate classification. With a reliable survey, one could further investigate which are the main spectral features that must be considered for differentiating between objects photoionized by hot $\mathrm{O}$ and $\mathrm{B}$ stars from those photoionized by non-thermal powerlaw continua.

There are several different methods for identifying AGNs in surveys. All of them consider particular features of AGN spectra that make these objects unique, such as the presence of strong or broad emission lines, an unusually blue continuum, or strong radio, infrared or high-energy emission. Even though these methods are effective, each also comes with their selection effects. In nearby galaxies, it is possible to detect weak AGNs if one takes care to correctly subtract the stellar features to isolate any non-stellar signal from the nucleus that could be weak in comparison to the host galaxy signal. Therefore, the most effective and least biased method to detect nearby AGNs, in the optical domain, is by conducting a spectroscopic survey of a complete, optical-flux limited sample of galaxies (Ho, 2008). This strategy is especially interesting because it does not solely detect AGNs, but simultaneously produces samples of non-AGN galaxies, including star-forming and quiescent galaxies. In order to obtain reliable measurements of the intensities of the most 
important lines, such a survey should be deep and cover the optical window from the [O II] doublet at $\sim 3727 \AA$ to the $[\mathrm{S} \mathrm{II}]$ doublet at $\sim 6730 \AA$ in the rest-frame with sufficient spectral resolution ( $R$ of at least several thousands).

In order to calculate the emission line ratios that are used to classify the objects, the data must have accurate relative flux calibration. In the case of integrated spectra of galactic nuclei, the information gathered in the slit includes the contribution from the starlight, the nebular line emission and any other emitting source that is inside the aperture. Therefore, the adequate measurement of emission-line flux intensities of any non-stellar components in galactic nuclei is only feasible if one applies a robust scheme to account for the stellar absorption-line spectrum to properly subtract the contamination by starlight (Kauffmann et al., 2003).

Given the recent advent of accurate and extensive surveys, the astronomical community has seen a rapid increase of large amounts of data that facilitates large-scale statistical studies of the Universe (Meléndez et al., 2014). The ideal sample for accomplishing the main goals of this work must fulfill the following criteria:

- Optical spectral range: adequate spectral range, in the optical domain, with the intensities of the emission lines measured and the respective errors of such measurements;

- Complete and uniform: the sample should be obtained with the same procedures and equipment to provide the data in order to make statistical analysis reliable;

- Local Universe: objects at low redshift to guarantee that the nuclei of the galaxies are observed with the highest possible resolution with the available equipment;

- Open source data: offers the opportunity to compare with other works in the literature that have done similar analysis considering the same data.

Based on these criteria, the Sloan Digital Sky Survey (SDSS; York et al., 2000) is the one that suits our purposes the best. The SDSS has been widely used for studies of emission-line galaxies (e.g., Kauffmann et al., 2003; Kewley et al., 2006; Stasińska et al. 2006; Schawinski et al., 2007; Cid Fernandes et al., 2010) and has proven to be a powerful resource for better understanding the physical properties and chemical composition of galaxies in the local Universe (Tremonti et al., 2004). The results of this survey are based 
on a large number of objects with a clear selection function, medium resolution spectra and elaborate subtraction of stellar features, resulting in a large amount of good quality, homogeneously obtained spectra (Stasińska et al., 2006). More details on this important survey are given in the next section.

\subsection{Sloan Digital Sky Survey}

The main goal of the Sloan Digital Sky Survey (SDSS; York et al., 2000) was to provide data that could support investigations of the distribution of luminous and nonluminous matter in the Universe by the production of imaging and spectroscopic surveys over a large area of the sky. To accomplish this task, the SDSS consisted of an imaging survey in five photometric bands of $\pi$ steradians in the North Galactic Cap (with $2 \%$ rms errors or better in photometric and astrometric calibration), and a follow-up spectroscopic survey of roughly $10^{6}$ galaxies and $10^{5}$ quasars, complete within precisely defined selection criteria. As further explained in more details, the spectroscopy was performed through 3-arcsec diameter fibres with a wavelength range of 3800 to $9200 \AA$ and a mean spectral resolution of $\sim 1800$. From the Seventh Data Release (DR7; Abazajian et al., 2009) onwards, the survey shifted its focus to observing stars and monitoring supernovae.

The SDSS imaging database contains photometric information of all detected objects (with positions, magnitudes, shape parameters, model fits and processing flags), atlas images of all detected objects and cross-matches with other catalogs (such as FIRST and ROSAT), a $4 \times 4$ binned image of the corrected frames with the objects removed, and a mask of the areas of non processed sky and of corrected pixels. The spectroscopic database contains all spectroscopic information, including the calibrated one-dimensional spectra, the derived redshift and the spectral type of the objects, and the detailed log for the spectroscopic observations.

In order to obtain these data, the survey used a dedicated telescope equipped with a large-format mosaic CCD camera to image the sky in five optical bands, and two digital spectrographs to obtain 640 spectra simultaneously for objects selected from the imaging data. The telescope is a $2.5 \mathrm{~m} \mathrm{f/5}$ modified Ritchey-Chrétien wide-field altitude-azimuth instrument (Gunn et al. 2006) located at the Apache Point Observatory in Sunspot, New Mexico. Its photometric/astrometric mosaic camera scans the sky along large circles and 
is mounted at the Cassegrain focus. It is a wide-field imager (Gunn et al., 1998) with $242048 \times 2048$ CCDs on the focal plane that cover the sky in five filters, in the order riuzg (Fukugita et al., 1996). The effective exposure time per filter is $54.1 \mathrm{~s}$, and $18.75 \mathrm{deg}^{2}$ were imaged per hour in each of the five filters, mostly taken under good seeing conditions on moonless photometric nights (Hogg et al., 2001).

The telescope also used two double fiber-fed multi-object spectrographs on the image rotator. Each fiber has entrance diameter of 3", and they must not flex nor be placed closer than 55" apart on any given plate. The mentioned characteristics were chosen in order to allow simultaneous spectroscopy of essentially all the galaxies in a given field of view to the faintest magnitude for which the used telescope could measure redshifts in a reasonable amount of time. The several spectroscopic exposures were 15 minutes long, and three or more were taken for a given plate to reach the predefined requirements of $(\mathrm{S} / \mathrm{N})^{2}>15$ per $1.5 \AA$ pixel for stellar objects of fiber magnitude $g=20.2, r=20.25$, and $i=19.9$ AB mag. The wavelength range of the spectra is 3800-9200 $\AA$ over 4098 pixels, and the resolution $\lambda / \Delta \lambda$ is between 1850 and 2200 .

Besides the $2.5 \mathrm{~m}$ telescope, the SDSS made use of three subsidiary instruments at the site, which are the photometric telescope (a $0.5 \mathrm{~m}$ telescope equipped with a CCD camera and the SDSS filter set) to calibrate the photometry, the seeing monitor and the $10 \mu \mathrm{m}$ cloud scanner to monitor the astronomical weather.

\subsection{Specifications of the sample used in this thesis}

This section will specify the criteria considered in this work in order to produce a sample of galaxies from the SDSS data that will be used to address the scientific questions related to the taxonomy of galactic nuclei studied in this thesis.

In order to carry out measurements of the distributions of galaxy properties with the highest possible precision, the sample of galaxies for which spectra are taken should be selected in a uniform and objective manner. The Main Galaxy Sample (Strauss et al., 2002) uses such selection with the cumulative results from the data releases of objects spectroscopically classified by SDSS as galaxies given by the Seventh Data Release Abazajian et al., 2009). This main spectroscopic galaxy sample is a reddening-corrected $r$-band magnitude-limited sample of galaxies brighter than $r_{P}=17.77$ mag, with an estimated 
surface density of 92 galaxies per square degree and a median redshift of 0.104 . The magnitude is measured within a Petrosian aperture, so as to provide a meaningful measure of a fraction of the total light of the galaxy that is independent of distance to the galaxy, reddening, and sky background. The SDSS spectra are of high enough signal-to-noise ratio $(\mathrm{S} / \mathrm{N}>4$ per pixel) that essentially all targeted galaxies $(99.9 \%)$ yield a reliable redshift (i.e., with statistical error less than $30 \mathrm{~km} \mathrm{~s}^{-1}$ ). Star-galaxy separation is based on the difference between the point spread function (PSF) and galaxy model magnitudes, which effectively quantifies the extensiveness of the source relative to the PSF.

The galaxies targeted spectroscopically by the SDSS constitute a uniform sample spanning a wide range of galaxy types, ideal for analyses of large-scale structure and galaxy properties. However, no sample is totally free of selection biases, as will be discussed further in the analysis of this work. It is worth mentioning that the Main Galaxy Sample does not include objects with (obvious) broad lines such as present in Seyfert 1 galaxies, which are considered "QSOs" in the spectroscopic SDSS classification nomenclature.

The data used in this thesis are the measurements from the Max Planck Institute for Astrophysics and the Johns Hopkins University (MPA-JHU) ${ }^{1}$ value-added catalog, based on the methods detailed in Brinchmann et al. (2004) and Tremonti et al. (2004). For the analysis of the objects in our sample, we considered the emission-line fluxes, equivalent widths and continuum flux densities with their respective errors, and basic information about the objects such as their position in the sky, plate, fiber and photo ID (to identify outliers and check their photometric information), the redshift, and the velocity dispersion.

The Seventh Data Release of SDSS with the selection of the Main Galaxy Sample is very useful to study local galactic nuclei. The sample is uniform and complete, even though the criteria for this selection (limiting magnitude and consequently a limited redshift range) should be kept in mind as its main limitations. The spectra have high quality with regard to $\mathrm{S} / \mathrm{N}$, focuses on local objects, and the wavelength coverage is sufficient to study the optical emission lines necessary to classify galactic nuclei, which are $\mathrm{H} \beta$, [O III] $]$ 5007, [O I] $\lambda 6300$, $\mathrm{H} \alpha,[\mathrm{N}$ II] $\lambda$ 6584, and [S II] $\lambda \lambda 6717,6731$ (Baldwin et al., 1981; Veilleux and Osterbrock, 1987).

From the thousands of galaxies with spectra from SDSS DR7, we defined our sample by applying redshift $(z)$ and error $(\epsilon)$ cutoffs to guarantee high quality data of the spectra

\footnotetext{
${ }^{1}$ Available at https://wwwpa.mpa-garching.mpg.de/SDSS/DR7/raw_data.html.
} 
being studied. The resulting sample consists of 4192 galaxies at redshift $z<0.016$ and with the errors of all the emission line ratios considered in the traditional diagnostic diagrams $\epsilon<0.1$ dex. These criteria minimize the scatter of the data in the diagnostic diagrams and the chances of objects being missclassified.

The redshift cut is necessary for ensuring that the properties derived from the fixedsize slit of SDSS spectra pertain to the nuclei, minimizing the possible contamination by H II regions nearby the nucleus or even in the outskirts of the host galaxy. Without this redshift cut, the SDSS spectra tend to represent much better the global galaxy properties than those of the nuclei (Kauffmann et al., 2003). This would result in data more contaminated by stellar and nebular light from outside the nuclear region, confusing the possible classification of the nuclei and thus leading to systematic effects that are hard to quantify (Ho, 2008). The redshift cut was further motivated by the redshift above which the $[\mathrm{S}$ II] $\lambda \lambda 6717,6731$ doublet shifts into the $6850 \AA$ atmospheric band. Considering the cosmological parameters of a flat Universe with a Hubble constant of $\mathrm{H}_{0}=69.6 \mathrm{~km} \mathrm{~s}^{-1}$ $\mathrm{Mpc}^{-1}$, a matter density $\Omega_{\mathrm{M}}=0.286$ and a vacuum energy density $\Omega_{\mathrm{VAC}}=0.714$, the maximum diameter of the nuclear area of a galaxy in our sample $(z=0.016)$ is $\sim 990 \mathrm{pc}$ (Wright, 2006). Hence, the maximum diameter of the observed regions is around $1 \mathrm{kpc}$.

The error criteria of $<0.1$ dex on all the line ratios were proposed in order to select only high quality data and guaranteeing that the results of this work are robust. However, as shown by Salim et al. (2014), the selection criteria based on these emission lines simultaneously lead to a preferential removal of galaxies with lower specific star formation rates and higher metallicities. Nevertheless, since the purpose of this thesis is not to make a demographic study, we maintain the error criteria with consideration of its caveats.

The diagnostic diagrams shown hereafter, both traditional and alternative, are presenting our data sample according to the different classification schemes that are discussed in Section 3.1.1. 
Chapter 3

\section{Analysis}

After having introduced the standard diagnostic diagrams (BPT, VO-S, and VO-O) in Section 1.2.1 and presented the data sample that will be considered in our analysis in Section 2.3, we will now study how these data behave in the diagnostic diagrams in Section 3.1 below. After introducing the data as a whole, we will discuss how our objects would be classified in terms of their dominant power source for some of the most popular classification schemes from the literature, in Section 3.1.1. Next, we will enter into further detail about the objects that lie in between the various main classes (known as transition objects) and about the misclassifications that occur when using only the three traditional diagnostic diagrams, in Sections 3.1.2 and 3.1.3, respectively.

Having so acquired a basic understanding of the standard diagnostic diagrams and their usage, we will then analyze a set of alternative diagnostic diagrams and associated classification borderlines, in Section 3.2 .

Last, we will discuss theoretical models for the spectra of galactic nuclei in Section 3.3 . starting with the stellar components in Section 3.3.1 and then the parameters to produce our photoionization models in Section 3.3.2. Section 3.3.3 will go into further details about the nitrogen abundance and its relevance for modeling adequately the positions of sources in the diagnostic diagrams. Then, in Section 3.3.4 we will analyze the behaviour of our models in the diagnostic diagrams, considering both the traditional and our alternative classification schemes. 


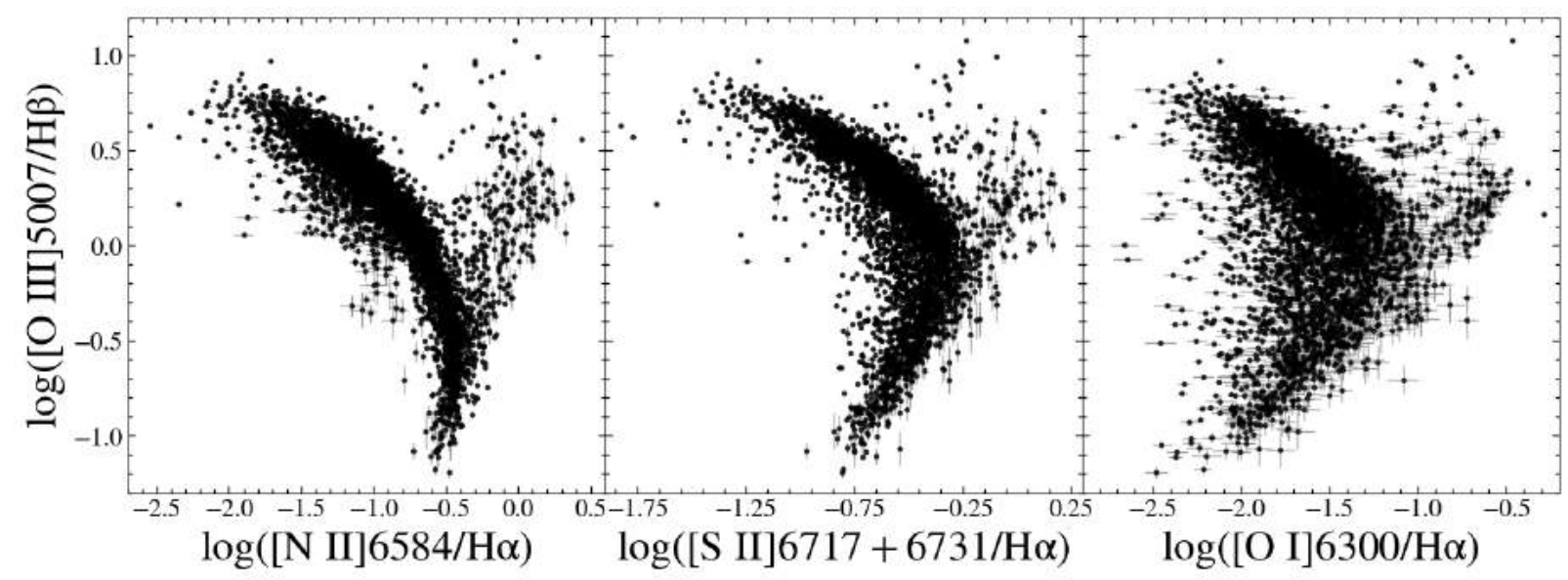

Figure 3.1: BPT (left), VO-S (middle), and VO-O (right) diagnostic diagrams for all of the objects from the SDSS DR7 Main Galaxy Sample with $z<0.016$ and $\epsilon<0.1$ dex.

\subsection{Traditional diagnostic diagrams}

As demonstrated in Section 1.2, two-dimensional classification schemes considering emission-line ratios as their axes are a powerful tool to probe the nature of the ionizing and excitation sources in galaxies. These diagnostic diagrams are able to separate the objects based on the hardness of their spectra from the intensity of their line ratios, placing nebular emission from gas photoionized by hot stars along a different locus of the diagram than gas photoionized by the activity of a SMBH. The diagrams are also able to separate the AGN that present high-ionization levels (such as Seyferts) from those that present relatively low levels of ionization (such as LINERs). Regions of the diagram that might contain objects with both photoionization from star formation and from non-stellar processes will be discussed in further detail in Section 3.1.2, after having presenting the main classification schemes in Section 3.1.1.

We will start our analysis by presenting the galactic nuclei selected for our sample (described in Section 2) in the traditional "BPT", "VO-S", and "VO-O" diagnostic diagrams, shown in Figure 3.1 in the left, middle and right panels, respectively. The objects in the diagnostic diagrams are distributed in two main branches as seen in Figure 3.1, and this shape is often referred to as a "seagull" (Stasińska et al., 2006). The left wing of the seagull is composed of star-forming objects, lying along a narrow sequence, while the right wing is wider and populated by objects that are photoionized primarily by non-stellar sources, reaching higher values of $[\mathrm{N} \mathrm{II}] / \mathrm{H} \alpha$. These features are a result of the gas-phase abundance, the physical conditions of the ISM, and the processes that drive the ionization of nebular 
regions in galactic nuclei (Agostino and Salim, 2019). The seagull shape is clearest in the BPT diagram of the left panel, but can also be seen in the VO-S and VO-O diagrams, as shown the middle and right panels of Figure 3.1.

The forbidden emission lines considered in the VO-S diagram in the middle panel of Figure 3.1 are sensitive to the electron temperature and the electron density of the nebula (Lequeux et al., 1979; Osterbrock and Ferland, 2006). As for the line ratios, both are sensitive to the ionization parameter. The line ratio of $[\mathrm{O} \mathrm{III}] / \mathrm{H} \beta$ can be considered an ionization parameter diagnostic by itself, since the presence of $\mathrm{O}^{++}$requires a high excitation source (Groves et al., 2004). The [S II] lines are mainly produced in the partially ionized/partially neutral zone located at the edge of the ionized nebula, and the length of this zone depends on the ionization parameter. Hence, the line ratio of $[\mathrm{S} \mathrm{II}] / \mathrm{H} \alpha$ is strongly dependent on the ionization parameter as well, besides its metallicity (Kewley et al., 2019).

The VO-O diagram shown in the right panel of Figure 3.1 presents the largest scatter, as expected from the difficulty in measuring the weak line [O I] confirmed by larger errors in the abscissa. Nevertheless, it presents a similar trend of AGNs showing stronger $[\mathrm{O} \mathrm{I}] / \mathrm{H} \alpha$ ratios (right branch) than star-forming regions (left branch).

In comparison with other works that used SDSS data in the traditional diagnostic diagrams (e.g., Kauffmann et al., 2003; Kewley et al., 2006; Stasińska et al., 2006; Schawinski et al., 2007; Cid Fernandes et al., 2010), the right-hand branches in Figure 3.1 have fewer objects, and the overall diagrams are less populated. This is a consequence of our redshift cut and the fact that this work considered an error threshold of $\epsilon<0.1$ on all of the line ratios. This criterion was adopted in order to guarantee that objects are not occupying a certain region of the diagrams because of large uncertainties, such that the alternative diagnostic diagrams that will be proposed later in Section 3.2 are based on data with high accuracy. Thus, we are aware of the selection effects affecting our sample and consequently the distribution of the objects in the diagnostic diagrams. However, we do not intend to give a representative picture of the Local Universe, and our main goal is to propose useful alternative diagnostic diagrams. In view of that, it is most important to have reliable data extracted from high quality spectra. For completeness, we mention that the WHAN diagram proposed by Cid Fernandes et al. (2011) and mentioned in Section 1.2.2 is suitable also for lower quality spectral data.

The left-hand branch of the diagnostic diagrams is populated by objects with ongoing 
star formation, where the nebular gas is primarily ionized by hot $\mathrm{O}$ and $\mathrm{B}$ stars. This has been confirmed by models (e.g., Kewley et al., 2001; Nagao et al., 2006, Stasińska et al. 2006; Levesque et al., 2010), and the clean sequence shown in the diagrams (denominated star-forming sequence) is mainly a sequence in metallicity of the ionized gas (Dopita et al. 2006; Vale Asari et al., 2007). Other parameters such as the ISM pressure, the hardness of the ionizing radiation field, and the ionization parameter also influence in the position of an object (Kewley et al., 2019). In the BPT diagram of Figure 3.1, the star-forming galaxies with higher mass and thus, on average, a higher metallicity are preferentially found in the lower part of the branch, showing low $[\mathrm{O}$ III $] / \mathrm{H} \beta$ and high $[\mathrm{N} \mathrm{II}] / \mathrm{H} \alpha$ Vale Asari et al., 2007; Stasińska et al., 2008). On the other hand, star-forming galaxies with high $[\mathrm{O} \mathrm{III}] / \mathrm{H} \beta$ and low $[\mathrm{N} \mathrm{II}] / \mathrm{H} \alpha$ ratios tend to have lower mass and lower metallicity, occupying the upper left part of the BPT diagram (Kewley et al., 2013).

The AGN branch is positioned in the upper right of the diagrams, as one would expect from their high ionization parameter and hard radiation field, that produce more intense emission lines of higher levels of ionization than the ones that can be achieved with pure stellar processes. This intense radiation field in AGNs is explained by the production of high energy photons associated with the accretion of matter. These highly energetic photons heat the NLR gas through photoionization. This releases hot electrons that raise the kinetic temperature of the gas through Coulomb interactions with thermal electrons, and consequently the collisionally excited lines will emit with higher intensities in relation to Balmer recombination lines (Stasińska et al., 2006; Agostino and Salim, 2019). The AGN branch rises from the bottom part of the star-forming branch because luminous AGNs tend to reside in the more massive and metal-rich galaxies (Stasińska, 1984; Storchi-Bergmann and Pastoriza, 1990; Storchi-Bergmann et al., 1998; Kauffmann et al., 2003; Schawinski, 2009; Feltre et al., 2016; Thomas et al., 2019).

It should be noted that while in the star-forming sequence we have only $\mathrm{H}$ II region-like objects, the right branch of the diagnostic diagrams includes also objects that are not photoionized by an AGN. The presence of shocks contributes to the high intensities of the emission line ratios, and the higher the shock velocity, the higher $[\mathrm{O}$ III] $] / \mathrm{H} \beta$ (Spence et al. 2016; Dors et al., 2021). The fastest shocks, however, are still linked to AGN activity, usually as a result of radio jets and/or outflows from the ISM. A more problematic type of contamination on or near the AGN branch of the standard diagnostic diagrams are galaxies 
whose emission lines are produced by hot post-AGB stars and white dwarfs. According to the models from Stasińska et al. (2008), galactic nuclei dominated by old stellar populations are found in the lower right part of the diagrams, overlapping with LINERs.

For both classes of objects, photoionization models have shown that local galaxies with higher $[\mathrm{O}$ III $] / \mathrm{H} \beta$ and higher $[\mathrm{N} \mathrm{II}] / \mathrm{H} \alpha$ ratios have a larger ionization parameter than the objects in other loci of the diagrams (Pérez-Montero et al., 2019; Kewley et al., 2013). This can be due to higher electron densities and larger escape fraction of photons with energies higher than $13.6 \mathrm{eV}$, capable of ionizing hydrogen (Brinchmann et al., 2008).

Considering a comparison between the three diagrams from Figure 3.1, only the BPT diagram on the left clearly shows the shape of the seagull with open wings. This is a consequence of the coupling between the chemical abundances $\mathrm{N} / \mathrm{O}$ with regard to $\mathrm{O} / \mathrm{H}$, the ionizing radiation field hardness, and the ionization parameter, that drive the intensities of [N II] and [O III] (McCall et al., 1985; Dopita and Evans, 1986; Stasińska et al., 2006; Cid Fernandes et al. 2010). The nitrogen abundance is not uniquely determined as a constant fraction of the total abundance, unlike most other elements. This is due to the fact that nitrogen has both primary and secondary productions (Alloin et al. 1979), that will be discussed in more detail in Section 3.3.3.

The objects that lie in between the two well defined loci (part of the body of the seagull) are often called composite or transition objects, and their sequence is referred to as a mixing sequence, with the mixture of star formation and AGN activity increasing with increasing line ratios. This subject will be further discussed in Section 3.1.2. However, we will first discuss the classification schemes that separate quantitatively the objects among each of the possible classes, in Section 3.1.1.

\subsubsection{Object classification}

The standard diagnostic diagrams are quite useful because of the visual distinction between each type of object based on their main ionization source. However, in order to use them as tools for galactic nuclei classification, it is interesting to define quantitative distinctions between the various loci.

Veilleux and Osterbrock (1987) already proposed a boundary based on theoretical models. However, the parametrization of their division line is not explicit in the paper. Moreover, the amount of available and reliable data of photoionized objects grew substantially 


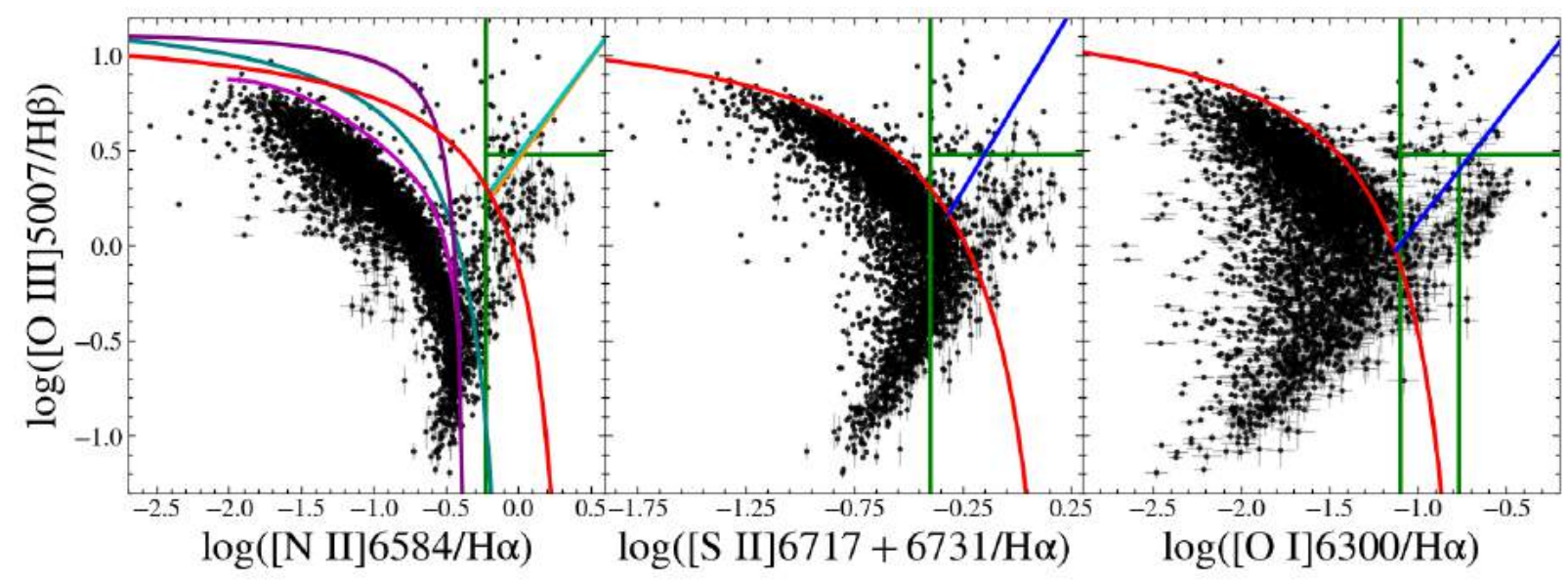

Figure 3.2: BPT (left), VO-S (middle), and VO-O (right) diagrams with all the proposed borderlines. Black dots indicate all the data from our sample. Magenta curve from Stasińska et al. (2006), teal curve from Kauffmann et al. (2003), purple curve from Meléndez et al. (2014), red curve from Kewley et al. (2001), green lines from Ho, Filippenko \& Sargent (1997), cyan line from Cid Fernandes et al. (2010), orange line from Schawinski et al. (2007), blue line from Kewley et al. (2006).

from the 1980s to the 2000s, especially after the release of SDSS.

In this Section, we will introduce the main classification schemes that have been used in the literature. Some are empirical (e.g, Ho et al., 1997; Kauffmann et al., 2003; Schawinski et al., 2007), some purely theoretical (e.g., Kewley et al., 2001; Meléndez et al., 2014), and some take into account both approaches (e.g., Kewley et al., 2006, Stasińska et al., 2006 Cid Fernandes et al., 2010). Other classification schemes that consider the [O II] line have been proposed, for example (Lamareille et al., 2004), but those will not be considered in this thesis.

A summary of the main characteristics of each of the classification schemes that are introduced in this Section is presented in Table 3.1.1. The Table shows which of the diagnostic diagrams were used for the classification (columns 2-4), which objects the classification is sensitive to (columns 5-6), and if it is based on empirical or theoretical approaches (columns 7-8). All these classifications systems are also highlighted in the diagrams of Figure 3.2 .

Below we will describe each of the methods listed in Table 3.1.1 one by one (Subsections 3.1.1.1 to 3.1.1.8), and illustrate these classification schemes using our own sample. In the final Subsection 3.1 .1 .9 we will discuss these results. 
Table 3.1 - Summary of the classification schemes discussed in Section 3.1.1. The first column gives the paper where the classification scheme was presented (Ho97 for Ho, Filippenko \& Sargent 1997, Ke01 for Kewley et al. 2001, Ka03 for Kauffmann et al. 2003, Ke06 for Kewley et al.|2006, St06 for Stasińska et al. 2006, Sc07 for Schawinski et al. 2007, CF10 for Cid Fernandes et al. 2010, and Me14 for Meléndez et al. 2014). The next three columns indicate to which diagnostic diagrams the classification schemes are related (BPT as the left, VO-S as the middle, and VO-O as the right panel of Figure 3.1). The fifth and sixth columns indicate if the classification scheme segregates star-forming nuclei and AGN (SF-AGN) or the two ionization states of AGNs (Seyfert-LINER). The last two columns indicate if the classification scheme has an empirical or theoretical basis (can be both).

\begin{tabular}{|l|l|l|l|l|l|l|l|}
\hline Paper & BPT & VO-S & VO-O & SF-AGN & Seyfert-LINER & Empirical & Theoretical \\
\hline Ho97 & Yes & Yes & Yes & Yes & Yes & Yes & No \\
\hline Ke01 & Yes & Yes & Yes & Yes & No & No & Yes \\
\hline Ka03 & Yes & No & No & Yes & No & Yes & No \\
\hline Ke06 & Yes & Yes & Yes & Yes & Yes & Yes & Yes \\
\hline St06 & Yes & No & No & Yes & No & Yes & Yes \\
\hline Sc07 & Yes & No & No & No & Yes & Yes & No \\
\hline CF10 & Yes & No & No & No & Yes & Yes & Yes \\
\hline Me14 & Yes & No & No & Yes & No & No & Yes \\
\hline
\end{tabular}

\subsubsection{Ho, Fillipenko \& Sargent 1997}

The work from Ho, Filippenko \& Sargent (1997) was among the first in proposing equations that distinguish the dominant power source in extragalactic objects based on the standard diagnostic diagrams. However, this paper contains much more relevant information to the study of nuclei of nearby objects, since it is related to the Palomar Survey.

The Palomar Survey was an optical spectroscopic survey of the nuclear regions of a sample of nearby galaxies from the Northern Hemisphere, providing a large number of uniform observations that allowed statistical inferences of these objects. The survey was based on long-slit observations with the Hale $5 \mathrm{~m}$ telescope in two optical ranges, $\sim 4230$ $5110 \AA$ (blue) and $\sim 6210-6860 \AA$ (red), with spectral resolutions of approximately $4 \AA$ and $2.5 \AA$, respectively. The survey observed almost every bright galaxy in the northern sky. The main focus was to search and study low-luminosity AGNs that were not in the Markarian catalog that was widely used until then. The scientific initiation project from which this master project originated was based on the Palomar Survey (Aydar and Steiner, 2016).

Ho, Filippenko \& Sargent (1997) describes the procedure for removing starlight contribution from the observed spectra and presents a catalog with the measurements of the 
spectra of 486 galaxies. The main spectroscopic parameters presented are related to the emission lines (intensity ratios, fluxes, profile widths, equivalent widths), the stellar absorption lines and the stellar continuum. Relevant information from other catalogs is also provided, such as morphological type, distance, magnitudes and colors, etc.. A discussion about the uncertainties and error estimates is conducted, for it is in general difficult to evaluate such values because of the starlight-subtraction process that is necessary for measuring the emission line properties, and that often dominates the systematic errors in the line measurements. For the forbidden lines of $[\mathrm{N} \mathrm{II}]$ and $[\mathrm{S} \mathrm{II}]$, that are relatively bright in the red wavelength range and not strongly affected by stellar absorption, their intensities could be determined to an accuracy of $\sim 10 \%$ in the best cases. As for the [O I] line, that is also in the red and not too affected by stellar absorption, this line is much weaker and it is difficult to achieve errors less than 20\%-30\%. With regard to [O III] line, though it is a bright line that is also not strongly affected by absorption, the blue spectra of the Palomar Survey have lower sensitivity than the red spectra, in a way that the accuracy is $10 \%-20 \%$. The recombination lines of the Balmer series, however, are more impaired by the uncertainties, which become progressively larger from $\mathrm{H} \alpha$ to $\mathrm{H} \gamma$. This is mainly due to the superposition of the emission and absorption components and the fact that the strength of the absorption component depends strongly on the age of the stellar population, in such a way that the intensities of the hydrogen emission lines can vary considerably depending on the chosen template model. Another source of uncertainties in all spectra is the consequence of using a long-slit to observe objects that are known to have spatially extended and inhomogeneous narrow-line emission.

The authors also stress the importance of considering a classification system that is based only on spectroscopic criteria, instead of a combination of spectroscopic, photometric, and morphological characteristics, as was customary in older works. Those previous studies often could not be extended to low-luminosity nuclei, and therefore did not offer a complete perspective of the galactic nuclei activity (e.g., de Vaucouleurs and de Vaucouleurs, 1968). Likewise, considering line widths to classify objects (e.g., Weedman, 1970 , 1977; Balzano and Weedman, 1981; Shuder and Osterbrock, 1981) is problematic because such parameter correlates strongly with luminosity in active nuclei (Phillips et al., 1983; Whittle, 1985, 1992; Ho, 1996), in such a way that line width-cutoffs select objects based on their luminosities. 
Ho, Filippenko \& Sargent (1997) determine the main sources of ionization based on the traditional diagnostic diagrams proposed by Baldwin, Phillips \& Terlevich (1981) and Veilleux and Osterbrock (1987). What makes this method of considering two-dimensional line-intensity ratio diagrams a useful tool to classify objects is that the Strömgren sphere of a classical, ionization-bounded H II region makes a reasonably sharp transition between the ionized interior and the surrounding neutral medium. As a consequence, the partially ionized transition zone (where most of the low-ionization forbidden lines such as [O I], [S II], and to some extent [N II] are emitted) is rather limited in size. On the other hand, an AGNlike ionizing continuum has sufficient high-energy photons to sustain an extensive partially ionized zone in clouds optically thick to the Lyman continuum, therefore enhancing the intensities of the low-ionization lines relative to those seen in star-forming regions.

With an appropriate choice of an excitation indicator, such as $[\mathrm{O} \mathrm{III}] / \mathrm{H} \beta$, one can delineate the excitation sequence in $\mathrm{H}$ II regions and also divide AGNs into a high-excitation class (Seyferts) and a low-excitation class (LINERs). However, the separation between the classes did not have sharp, rigorously-defined boundaries, given the available amount of data.

Table 3.2 - Classification scheme based on optical emission-line ratios proposed by Ho, Filippenko \& Sargent (1997).

\begin{tabular}{|l|cccc|} 
Class & {$[\mathrm{O}$ III $] / \mathrm{H} \beta$} & {$[\mathrm{O}$ I $] / \mathrm{H} \alpha$} & {$[\mathrm{N} \mathrm{II}] / \mathrm{H} \alpha$} & {$[\mathrm{S} \mathrm{II}] / \mathrm{H} \alpha$} \\
\hline H II nuclei & Any & $<0.08$ & $<0.6$ & $<0.4$ \\
Seyfert nuclei & $\geq 3$ & $\geq 0.08$ & $\geq 0.6$ & $\geq 0.4$ \\
LINERs & $<3$ & $\geq 0.17$ & $\geq 0.6$ & $\geq 0.4$ \\
Transition nuclei & $<3$ & $\geq 0.08,<0.17$ & $\geq 0.6$ & $\geq 0.4$ \\
\hline
\end{tabular}

Hence, we can see from Table 3.2 that Ho, Filippenko \& Sargent (1997) considers Seyfert galaxies as objects with high values both of $[\mathrm{OIII}] / \mathrm{H} \beta(>3)$ and of other ratios involving lower ionization lines, such as $[\mathrm{N} \mathrm{II}] / \mathrm{H} \alpha,[\mathrm{S} \mathrm{II}] / \mathrm{H} \alpha$ and $[\mathrm{O} \mathrm{I}] / \mathrm{H} \alpha$, while LINERs have lower values of $[\mathrm{O} \mathrm{III}] / \mathrm{H} \beta(<3)$ and high values of ratios involving the lower ionization lines. The H il nuclei are characterized by low values of ratios involving the lower ionization lines (as explained by the small layer of partially ionized transition zone) and can present any value for $[\mathrm{OIII}] / \mathrm{H} \beta$. The classification scheme proposed also considers a class of transition objects, that are defined by $[\mathrm{O} \mathrm{I}] / \mathrm{H} \alpha$ strengths between those of typical starforming regions and LINERs. Such objects will be further discussed in Section 3.1.2. Among the $86 \%$ objects of the sample that contain emission-line nuclei, $49 \%$ are classified 


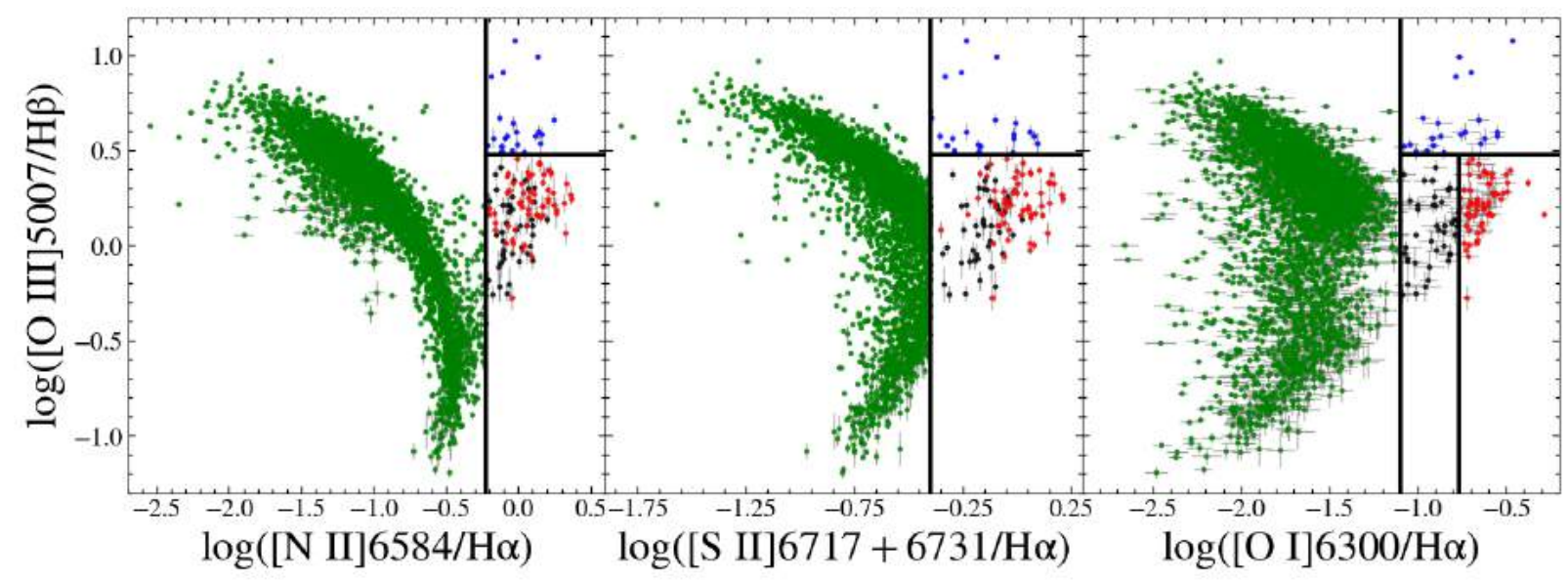

Figure 3.3: BPT (left), VO-S (middle), and VO-O (right) diagrams considering the classification scheme from Ho, Filippenko \& Sargent (1997) (see Table 3.2. Points in green are star-forming nuclei, in red are LINERs, in blue are Seyfert nuclei, and in black are transition objects.

as star-forming, 23\% as LINERs, $16 \%$ as transition objects, and $12 \%$ as Seyferts (compared to $96.52 \%, 1.7 \%, 1.19 \%$, and $0.59 \%$ in our sample that is not representative of a complete population of galactic nuclei; see Table 3.1.1.9 below). Ho, Filippenko \& Sargent (1997) already points out the main problem when considering three different diagrams to classify the objects, which is the possible ambiguity. This issue is will be discussed in more detail in Section 3.1.3.

Figure 3.3 shows the classification scheme from Table 3.2 applied to our sample. We can see that many of the objects are not classified due to the ambiguity issue. This effect can be seen more explicitly in the comparison of the VO-S diagram from the middle panels of Figure 3.1, with all the objects from the sample, and of Figure 3.3, where the distinction between the classes excludes a considerable part of $\mathrm{H}$ II nuclei with higher $[\mathrm{S} \mathrm{II}] / \mathrm{H} \alpha$, in a way that the seagull shape is interrupted. The transition nuclei (black points) are distinguished from the other classes considering only the VO-O diagram to the right, in a way that they overlap with LINERs in the other diagrams, but not with star-forming nuclei, which explains part of the missing data between the different classes. This effect of missing objects - probably as a consequence of the strong criteria regarding $[\mathrm{S} \mathrm{II}] / \mathrm{H} \alpha-$ is also seen in the right panel, as there seems to be a hole in the part that would be the body of the seagull, but that is still populated by star-forming regions.

It is worth recalling that this classification scheme was based on a sample with 486 galactic nuclei, by that time a considerable advance in big data. With this scheme, we were able to classify 3537 galactic nuclei from a total sample of 4192 objects ( $~ 84 \%)$. 
Ho, Filippenko \& Sargent (1997) emphasize that their classification system determines the probable ionization mechanism of the emission-line objects, and several caveats of the scheme are presented in the paper and pointed out in this Section.

\subsubsection{Kewley et al. 2001}

Kewley et al. (2001) presents models of star-forming regions to reproduce a large sample of infrared starburst galaxies, bringing a theoretical framework to the diagnostic diagrams. These models were then used to derive the first purely theoretical classification scheme to distinguish starburst and AGN galaxies based on optical diagnostic diagrams.

To make such models, as we will discuss further in Sections 3.3.1 and 3.3.2, SEDs from young star clusters were generated and used into photoionization codes to predict the emission lines of an ionized gaseous nebula. The contribution of shocks from supernovae was also modeled, but the conclusion is that their effects on the emission line ratios are negligible. The resulting models formed a grid with varying ionization parameter $(U$, see Equation 3.30 below) and metallicity $\left([Z]=Z / Z_{\odot}\right)$. However, modeled objects only populate a certain region of the standard diagnostic diagrams, even after accounting for variation in the gas-phase abundances and gas ionization state, in a way that only a harder ionizing radiation field could drive the objects to the region populated by AGNs.

In order to draw a theoretical upper limit for starbursts in the standard diagnostic diagrams, objects with the hardest EUV spectrum generated by the codes were chosen. Hence, Kewley et al. (2001) showed that, considering realistic ranges of metallicity $(0.1 \leq$ $[Z] \leq 3.0)$ and ionization parameter $(-3.5 \leq \log \mathrm{U} \leq-2.0)$, models with continuous star formation were always below and to the left of the theoretical boundary in all three traditional diagnostic diagrams. This is a consequence of the grid folding back upon itself, in a way that no combination of metallicity and ionization parameter could produce a synthetic object above this theoretical limit.

The lines proposed by Kewley et al. (2001) have the shape of rectangular hyperbolas, and present a 'maximum starburst line' for each standard diagnostic diagram. They are determined by the upper limit of the theoretical pure stellar photoionization models, hence, galaxies lying above these lines in each diagram are likely to have their power sources 


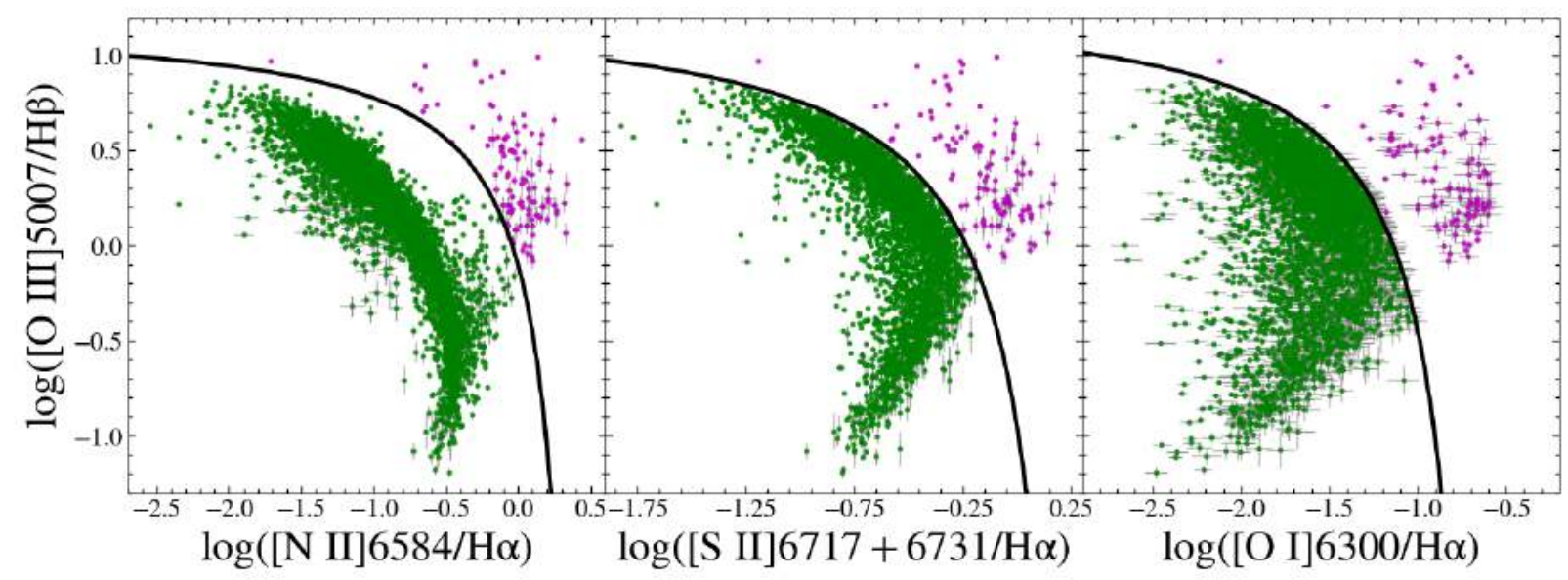

Figure 3.4: BPT (left), VO-S (middle), and VO-O (right) diagrams considering the classification scheme from Kewley et al. (2001) (see Equations 3.1 3.3). Points in green are star-forming nuclei and in magenta are AGNs (Seyferts and LINERs).

dominated by an AGN.

$$
\begin{aligned}
& \log \left(\frac{[\mathrm{O} \mathrm{III}]}{\mathrm{H} \beta}\right)=\left(\frac{0.61}{\log ([\mathrm{N} \mathrm{II}] / \mathrm{H} \alpha)-0.47}\right)+1.19, \\
& \log \left(\frac{[\mathrm{O} \mathrm{III}]}{\mathrm{H} \beta}\right)=\left(\frac{0.72}{\log ([\mathrm{S} \mathrm{II}] / \mathrm{H} \alpha)-0.32}\right)+1.30 \\
& \log \left(\frac{[\mathrm{O} \mathrm{III}]}{\mathrm{H} \beta}\right)=\left(\frac{0.73}{\log ([\mathrm{O} \mathrm{I}] / \mathrm{H} \alpha)+0.59}\right)+1.33 .
\end{aligned}
$$

According to these extreme starburst lines (Equations 3.1 3.3), 6\% of the sample considered by Kewley et al. (2001) with 285 infrared starburst galaxies showed ambiguous classifications, against $16 \%$ when considering the classification lines that where drawn by Veilleux and Osterbrock (1987). From an 'extreme mixing line' proposed to separate Seyferts, LINERs and H II region-like objects, Kewley et al. (2001) concluded that previous LINER classification schemes include not only bona fide LINERs, but also composite objects with both starburst and Seyfert characteristics.

Figure 3.4 shows this classification scheme applied to our data sample. The continuity in the seagull shape disappears, but more objects can be classified compared to Ho, Filippenko \& Sargent (1997). In the BPT diagram from the left panel, the borderline is more to the right than the seagull's shape, which reinforces the idea of an upper limit for the starforming cases. However, in both VO diagrams, from the middle and right panels, the lines closely follow the distribution of the data. This possible cut is more evident in the VO-O diagram to the right, where there seems to be a gap between the star-forming nuclei and 
the AGNs, in contrast with the more continuous shape observed with the whole sample in the right panel of Figure 3.1 .

Without considering the ambiguous cases, we were able to classify $\sim 93 \%$ of the total sample. In the cases where the classification schemes apply to all the diagnostic diagrams (as, for example Ho et al., 1997; Kewley et al., 2001, 2006), the price of having more reliable classification (since they depend simultaneously on all emission-line ratios) is that more objects that do not fit get excluded. Therefore, it is a choice of assuming the misclassification issue by considering the borderlines of only one diagram and checking the other diagrams (as we will see in the cases of Kauffmann et al., 2003, Stasińska et al., 2006; Schawinski et al., 2007; Cid Fernandes et al., 2010; Meléndez et al., 2014), or to only evaluate the objects that are not misclassified.

It is worth pointing out that the proposed maximum starburst line indicates that the SED responsible for producing the observed line ratios must be harder than the ones produced by stellar populations, due to activity associated with an accretion disk surrounding an active SMBH and/or shocks. It does not mean that the objects that lie below the line are necessarily purely star-forming galaxies, as will be further discussed in the next sections. Moreover, Kewley et al. (2001) suggests that the distance of an object from this maximum starburst line could be used to determine the fractional contribution of an AGN.

\subsubsection{Kauffmann et al. 2003}

Kauffmann et al. (2003) is the first paper to include a large number of galaxies from the SDSS in the standard diagnostic diagrams. Based on the empirical distribution, a borderline with the same rectangular hyperbole shape as in Kewley et al. (2001) is proposed.

The work from Kauffmann et al. (2003) examines the properties of 22623 galaxies that host a narrow-line AGN in the redshift range $0.02<z<0.3$ (entirely above the selection in our work). One important result from this analysis is that AGN in the whole range of luminosities tend to reside almost exclusively in massive galaxies having sizes, stellar surface mass densities, and concentrations similar to those of typical early-type galaxies. However, the most luminous AGNs showed younger mean stellar ages than both low-luminosity AGNs and early-type galaxies, plausibly explained as a consequence of feedback processes. Such young stellar populations are a general property of the AGNs with the strongest [O III] emission lines. 


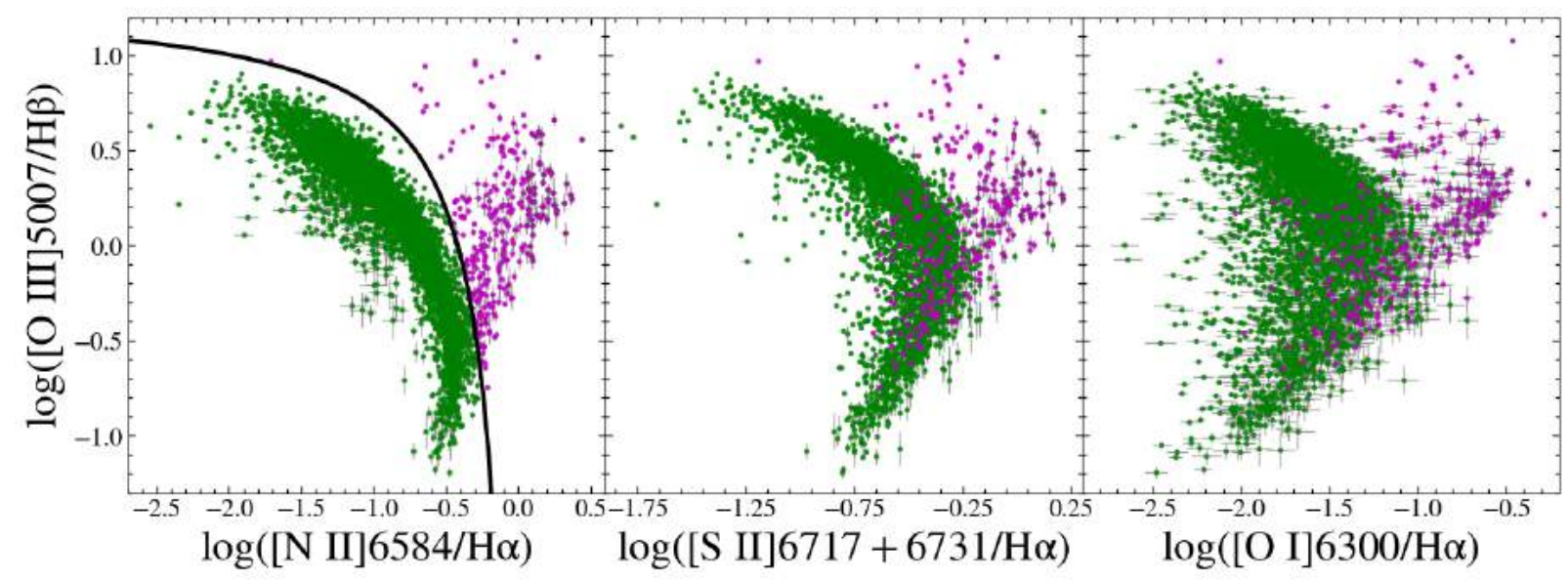

Figure 3.5: BPT (left), VO-S (middle), and VO-O (right) diagrams considering the classification scheme from Kauffmann et al. (2003) (see Equation 3.4). Points in green are star-forming nuclei and in magenta are AGNs (Seyferts and LINERs).

Based on the distribution of the data in the BPT diagram, Kauffmann et al. (2003) suggests a revision of the demarcation between starburst galaxies and AGNs that contours the left wing of the seagull with the following expression:

$$
\log \left(\frac{[\mathrm{O} \mathrm{III}]}{\mathrm{H} \beta}\right)=\left(\frac{0.61}{\log ([\mathrm{N} \mathrm{II}] / \mathrm{H} \alpha)-0.05}\right)+1.3
$$

By analyzing the position of thousands of objects in the diagnostic diagrams for the first time, it was possible to see a continuous behaviour between the objects photoionized by different sources. The region in between the extreme starburst line from Kewley et al. (2001) and the partition proposed by Kauffmann et al. (2003) is populated with composite objects, whose spectra contain significant contributions from both star formation and AGN activity. These transition objects (that will be further discussed in Section 3.1.2), that present line ratios intermediate between those of star-forming galaxies and LINERs or Seyferts, are probably an effect of the aperture size of the SDSS, since bona fide AGN spectra could be contaminated by surrounding star-forming regions. Though this combination with Kewley et al. (2001) is already proposed in Kauffmann et al. (2003), we have chosen to analyze only the classification scheme of Equation 3.4 in this Section and the transition object classification scheme will be analyzed in the following Sections.

Kauffmann et al. (2003) argues that the luminosity of [O III] could be a tracer of AGN activity, because though this line can be excited by massive stars as well, it will be typically weak in star-forming galaxies with high metallicities and high masses. In the star-forming sequence, as already discussed, as the metallicity of the objects increase, the $[\mathrm{N} \mathrm{II}] / \mathrm{H} \alpha$ 
ratio increases and the $[\mathrm{O} \mathrm{III}] / \mathrm{H} \beta$ decreases, so the green points in the bottom part of the left panel from Figure 3.5 represent the star-forming objects with higher metallicities. The AGN sequence "emerges as a plume" from the bottom of the locus of star-forming galaxies, and suggests that there is a mixing line in which the AGN contribution becomes stronger as we go to the upper right. As a consequence, the emission from gas ionized by star formation that contaminates bona fide AGNs classified as transition objects is related to metal-rich star-forming regions, in agreement with the fact that AGNs are mainly found in massive galaxies. Kauffmann et al. (2003) concludes that the $[\mathrm{O} \mathrm{III}] / \mathrm{H} \beta$ ratio is the best measure of AGN power in comparison to the other strong emission-line ratios of the traditional diagnostic diagrams, which justifies why this particular emission line ratio is used on the vertical axes in all three classical diagrams.

Kauffmann et al. (2003) proposed a borderline only for the BPT diagram (left), so we can analyze the quality of the classification method based on the misclassifications on VO-S and VO-O diagrams (middle and right, respectively), as discussed in the previous Section. Therefore, we are able to classify $100 \%$ of our data sample and analyze visually where the ambiguous objects lie in the remaining diagrams. This is useful to understand which classes can be confused, instead of simply not considering the ambiguous objects as was done in Ho, Filippenko \& Sargent (1997) and Kewley et al. (2001).

The magenta points (that represent AGNs) in the left branches of the middle and right panels from Figure 3.5 show that the borderline proposed by Kauffmann et al. (2003) in Equation 3.4 might be too strict, in a way that many of the objects that are classified as AGNs according to the BPT diagram to the left will actually lie in the star-forming branch of the VO-S and VO-O diagrams. The misclassified objects, however, are not in the loci of metal-poor star-forming galaxies, in agreement with Kauffmann et al. (2003).

This ambiguity emphasizes the need of considering all the different emission line ratios when proposing a classification scheme, since no single pair is able to completely distinguish between the power sources and all of them have their specific caveats. However, taking each of the classification schemes at a time might be interesting for not simply disregarding ambiguous objects.

\subsubsection{Kewley et al. 2006}

Kewley et al. (2001) and Kauffmann et al. (2003) led to a consensus presented in Kewley 
et al. (2006). In this work, a detailed study of sources in the SDSS along the right branch was developed, in a way that a partition line between high- (Seyferts) and low-ionization AGNs (LINERs) became possible in the VO-S and VO-O diagrams where their distinction is more evident. The resulting classification scheme separates the star-forming galaxies, composite objects (that present characteristics of both H II region and AGN), Seyferts and LINERs, and is widely used in the literature. The proposed borderlines are as follows.

Star-forming galaxies will be objects that have

$$
\begin{aligned}
\log \left(\frac{[\mathrm{O} \mathrm{III}]}{\mathrm{H} \beta}\right) & <\left(\frac{0.61}{\log ([\mathrm{N} \mathrm{II}] / \mathrm{H} \alpha)-0.05}\right)+1.3 \\
\log \left(\frac{[\mathrm{O} \mathrm{III}]}{\mathrm{H} \beta}\right) & <\left(\frac{0.72}{\log ([\mathrm{S} \mathrm{II}] / \mathrm{H} \alpha)-0.32}\right)+1.30 \\
\log \left(\frac{[\mathrm{O} \mathrm{III}]}{\mathrm{H} \beta}\right) & <\left(\frac{0.73}{\log ([\mathrm{O} \mathrm{I}] / \mathrm{H} \alpha)+0.59}\right)+1.33
\end{aligned}
$$

Composite galaxies (to be further discussed in Section 3.1.2) are defined based solely on the BPT diagram as

$$
\begin{gathered}
\log \left(\frac{[\mathrm{O} \mathrm{III}]}{\mathrm{H} \beta}\right)>\left(\frac{0.61}{\log ([\mathrm{N} \mathrm{II}] / \mathrm{H} \alpha)-0.05}\right)+1.3 \\
\log \left(\frac{[\mathrm{O} \mathrm{III}]}{\mathrm{H} \beta}\right)<\left(\frac{0.61}{\log ([\mathrm{N} \mathrm{II}] / \mathrm{H} \alpha)-0.47}\right)+1.19
\end{gathered}
$$

The Seyfert galaxies are those with

$$
\begin{aligned}
\log \left(\frac{[\mathrm{O} \mathrm{III}]}{\mathrm{H} \beta}\right) & >\left(\frac{0.61}{\log ([\mathrm{N} \mathrm{II}] / \mathrm{H} \alpha)-0.47}\right)+1.19 \\
\log \left(\frac{[\mathrm{O} \mathrm{III}]}{\mathrm{H} \beta}\right) & >\left(\frac{0.72}{\log ([\mathrm{S} \mathrm{II}] / \mathrm{H} \alpha)-0.32}\right)+1.30 \\
\log \left(\frac{[\mathrm{O} \mathrm{III}]}{\mathrm{H} \beta}\right) & >\left(\frac{0.73}{\log ([\mathrm{O} \mathrm{I}] / \mathrm{H} \alpha)+0.59}\right)+1.33
\end{aligned}
$$

or with

$$
\begin{array}{r}
\log \left(\frac{[\mathrm{O} \mathrm{I}]}{\mathrm{H} \alpha}\right)>-0.59, \\
\log \left(\frac{[\mathrm{O} \mathrm{III}]}{\mathrm{H} \beta}\right)>1.89 \log \left(\frac{[\mathrm{S} \mathrm{II}]}{\mathrm{H} \alpha}\right)+0.76, \\
\log \left(\frac{[\mathrm{O} \mathrm{III}]}{\mathrm{H} \beta}\right)>1.18 \log \left(\frac{[\mathrm{O} \mathrm{I}]}{\mathrm{H} \alpha}\right)+1.30 .
\end{array}
$$




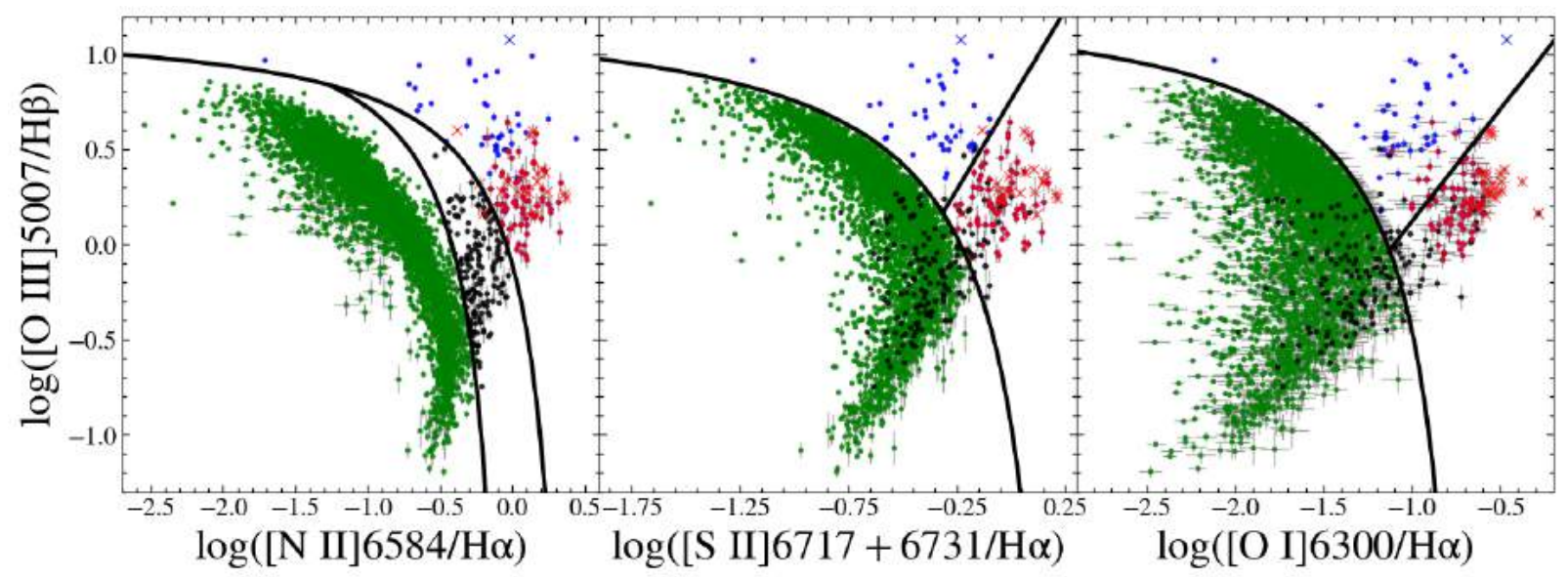

Figure 3.6: BPT (left), VO-S (middle), and VO-O (right) diagrams considering the classification scheme from Kewley et al. (2006) (see Equations 3.5 to 3.10). Points in green are star-forming nuclei, in blue are Seyferts (circles for Equations 3.7 and crosses for Equations 3.8), in red are LINERs (circles for Equations 3.9 and crosses for Equations 3.10, and in black are transition objects.

The low-ionization AGNs, LINERs, are determined by

$$
\begin{array}{r}
\log \left(\frac{[\mathrm{O} \mathrm{III}]}{\mathrm{H} \beta}\right)>\left(\frac{0.61}{\log ([\mathrm{N} \mathrm{II}] / \mathrm{H} \alpha)-0.47}\right)+1.19, \\
\log \left(\frac{[\mathrm{O} \mathrm{III}]}{\mathrm{H} \beta}\right)>\left(\frac{0.72}{\log ([\mathrm{S} \mathrm{II}] / \mathrm{H} \alpha)-0.32}\right)+1.30, \\
\log \left(\frac{[\mathrm{O} \mathrm{III}]}{\mathrm{H} \beta}\right)<1.89 \log \left(\frac{[\mathrm{S} \mathrm{II}]}{\mathrm{H} \alpha}\right)+0.76, \\
\log \left(\frac{[\mathrm{O} \mathrm{III}]}{\mathrm{H} \beta}\right)>\left(\frac{0.73}{\log ([\mathrm{O} \mathrm{I}] / \mathrm{H} \alpha)+0.59}\right)+1.33,
\end{array}
$$

or by

$$
\begin{array}{r}
\log \left(\frac{[\mathrm{O} \mathrm{I}]}{\mathrm{H} \alpha}\right)>-0.59 \\
\log \left(\frac{[\mathrm{O} \mathrm{III}]}{\mathrm{H} \beta}\right)<1.18 \log \left(\frac{[\mathrm{O} \mathrm{I}]}{\mathrm{H} \alpha}\right)+1.30
\end{array}
$$

Figure 3.6 presents the application of Equations 3.5 to 3.10 to the standard diagnostic diagrams. Since Seyferts and LINERs have two classification criteria, we have kept the same colors (Seyferts in blue and LINERs in red) and changed the marker symbols, with the objects classified by Equations 3.7 and 3.9 being presented as circles and those that considered Equations 3.8 and 3.10 as crosses.

Based solely on the BPT diagram to the left of Figure 3.6, we can see that the objects that lie below Kauffmann et al. (2003) line (Equation 3.4) are classified as star-forming galaxies, and those above Kewley et al. (2001) line (Equation 3.1) are classified as AGNs. Therefore, the galaxies that lie between the lines of Kewley et al. (2001) and Kauffmann 
et al. (2003) are denominated composite objects and belong to the 'AGN-H II mixing sequence', probably containing a metal-rich stellar population plus an AGN in their nuclei, that could be either a Seyfert-like or a LINER-like AGN. After the star-forming sequence from low metallicities (low $[\mathrm{N} \mathrm{II}] / \mathrm{H} \alpha$, high $[\mathrm{O} \mathrm{III}] / \mathrm{H} \beta$ ) to high metallicities (high $[\mathrm{N} \mathrm{II}] / \mathrm{H} \alpha$, low $[\mathrm{O} \mathrm{III}] / \mathrm{H} \beta)^{-}$, the 'AGN mixing sequence' begins at the high-metallicity end of the star-forming sequence and extends towards higher values of both line ratios. Seyferts have higher $[\mathrm{O}$ III $] / \mathrm{H} \beta$ line ratios than LINERs, but there is some overlap of LINERs showing high values of such line ratio (red data in the region of blue data) and fewer cases of the opposite misclassification.

As for the VO-S and VO-O diagrams from the middle and right panels of Figure 3.6, the lines proposed by Kewley et al. (2001) are valid for defining upper bounds to the starforming sequence. The objects that were classified as composite in the BPT diagram to the left are mainly in the star-forming loci of the VO-S diagram in the middle and more fairly distributed among star-forming galaxies and LINERs in the VO-O diagram to the right. There is no Seyfert galaxy in the loci of LINERs in either of the VO diagrams, though there are some LINERs in the Seyfert loci (red data in the region of blue data) in both, and to higher extent in the VO-O diagram. It is pointed out by Kewley et al. (2006) that the $[\mathrm{NII}] / \mathrm{H} \alpha$ line ratio is more sensitive to the presence of low-level AGN activity than the other low-ionization line ratios, due mainly to the metallicity sensitivity of the nitrogen line ratio.

The empirical division between Seyferts and LINERs is based on the two VO diagrams (see middle and right panels from Figure 3.6), where the two branches of AGNs are more clearly separated. However, because there are two classification criteria for those objects, we still see some overlap. Comparing the classification schemes that defined the colors of such objects with the BPT diagram we can see that the borderline is successful, with Seyferts in a top branch according to their higher ionization state than LINERs, which occupy a lower branch in the right wing of the seagull, as expected.

The work from Kewley et al. (2006) sums up the main conclusions from the previous works of the authors about this subject (Kewley et al., 2001; Kauffmann et al., 2003) and, based on the analysis of 85224 emission-line galaxies from SDSS, proposes an optical classification scheme that divides galactic nuclei into purely star-forming galaxies, Seyferts, LINERs, and composite AGN+star-forming galaxies. When we apply this scheme to our 
sample, we find that at least $\sim 94 \%$ is classified, with the remaining $6 \%$ having ambiguous classifications.

\subsubsection{Stasinska et al. 2006}

Stasińska et al. (2006) proposes a semi-empirical demarcation for normal star-forming galaxies and AGN hosts in the BPT diagram. It is semi-empirical because Stasińska et al. (2006) considers both observational data from SDSS and photoionization models. The work also proposes a method for classification of galactic nuclei based solely on the $[\mathrm{N} \mathrm{II}] / \mathrm{H} \alpha$ line ratio and an alternative diagram denoted DEW, which was mentioned in Section 1.2 .2 .

Based on photoionization models for star-forming regions and AGNs, Stasińska et al. (2006) derives an equation that correlates the ionization parameter and the metallicity and is able to reproduce the upper envelope of the left branch of the BPT diagram with the following expression

$$
\begin{array}{r}
\log \left(\frac{[\mathrm{O} \mathrm{III}]}{\mathrm{H} \beta}\right)=\left[-30.787+1.1358\left(\frac{[\mathrm{N} \mathrm{II}]}{\mathrm{H} \alpha}\right)+0.27297\left(\frac{[\mathrm{N} \mathrm{II}]}{\mathrm{H} \alpha}\right)^{2}\right] \times \\
\tanh \left[5.7409\left(\frac{[\mathrm{N} \mathrm{II}]}{\mathrm{H} \alpha}\right)\right]-31.093
\end{array}
$$

The expression above is valid only for $-2.0 \leq[\log (\mathrm{N} \mathrm{II}] / \mathrm{H} \alpha) \leq-0.4$ and shows a more restrictive separation between star-forming nuclei and AGNs when compared to Kauffmann et al. (2003), which itself was already more more restrictive than the theoretical maximum starburst limit proposed by Kewley et al. (2001). It is worth pointing out that this line was proposed before the classification scheme of Kewley et al. (2006).

Using these results, we could classify 4161 objects, equivalent to $\sim 99 \%$ of the whole sample. We maintained the same size for the axes as in the diagrams with the whole sample (see Figure 3.1) so the regions where there are missing objects (especially for the lowest values of $[\mathrm{N} \mathrm{II}] / \mathrm{H} \alpha$ in the left panel of Figure 3.7 and $[\mathrm{S} \mathrm{II}] / \mathrm{H} \alpha$ in the middle panel of Figure 3.7) are explicit.

Another behavior that was not seen in the classification schemes presented before is the misclassification of star-forming regions with low metallicity, in the upper part of the left branch, that are being misclassified as AGNs. Therefore, the magenta points that overlap the star-forming sequence on the VO diagrams are probably being misclassified by Equation 3.11, that refers solely to the BPT diagram. The restriction mainly excluded 


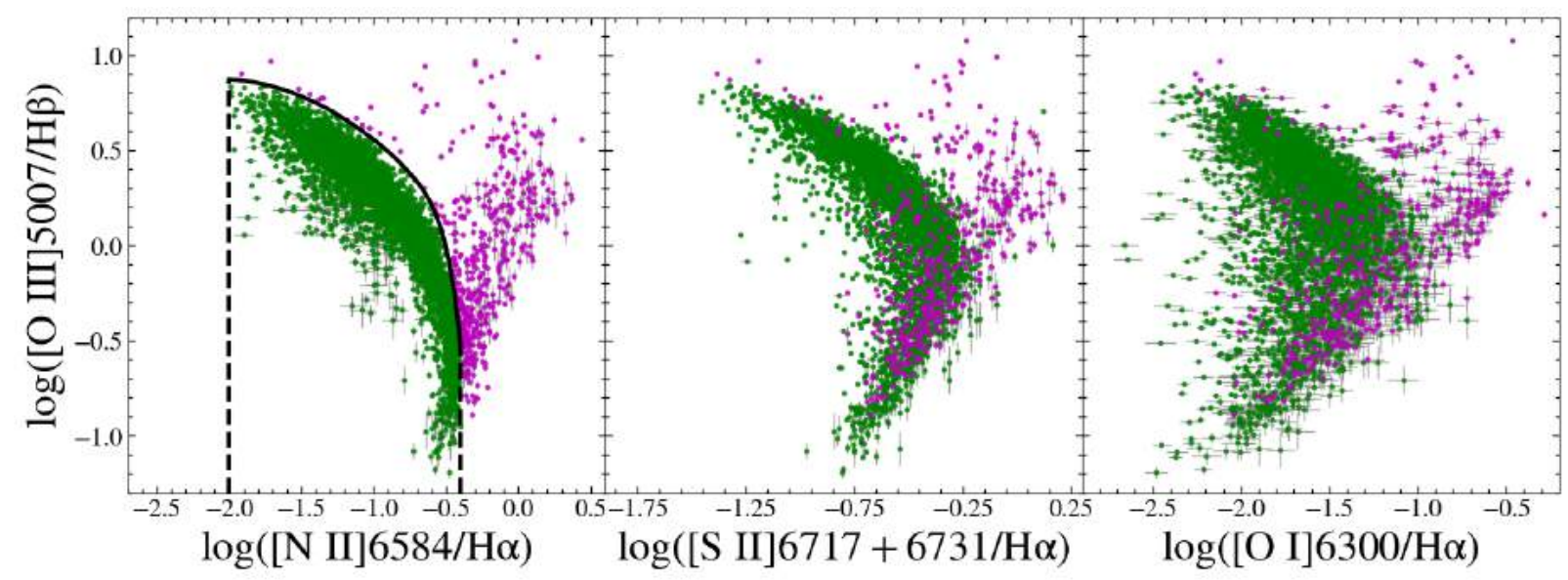

Figure 3.7: BPT (left), VO-S (middle), and VO-O (right) diagrams considering the classification scheme from Stasińska et al. (2006) in the filled line (see Equation 3.11). The dashed lines demarcate the limits where this classification is valid $(-2.0 \leq \log ([\mathrm{N} \mathrm{II}] / \mathrm{H} \alpha) \leq-0.4)$ in the left panel. Points in green are star-forming nuclei and in magenta are AGNs (Seyferts and LINERs).

star-forming nuclei with $\log ([\mathrm{N} \mathrm{II}] / \mathrm{H} \alpha)<-2.0$, and such low metallicity $\mathrm{H}$ II regions are not often confused in other classification schemes.

Hence, though the restriction of the borderline proposed by Stasińska et al. (2006) is based on theoretical models and also considers the SDSS data, in the comparison with the other standard diagnostic diagrams, it looks as if the line proposed earlier by Kauffmann et al. (2003) has fewer problems with ambiguous classifications of even misclassification. This kind of comparison between borderlines proposed based solely on the BPT being applied to VO-S and VO-O diagrams shows that such lines are usually too strict, as these latest diagrams do not account for a region of transition objects.

Stasińska et al. (2006) also proposes that, in the cases where $[\mathrm{O}$ III]/H $\beta$ cannot be measured, it is feasible to classify the nuclei solely based on $\log ([\mathrm{N} \mathrm{II}] / \mathrm{H} \alpha)$ :

$$
\begin{array}{r}
\log \left(\frac{[\mathrm{N} \mathrm{II}]}{\mathrm{H} \alpha}\right) \leq-0.4, \\
-0.4<\log \left(\frac{[\mathrm{N} \mathrm{II}]}{\mathrm{H} \alpha}\right) \leq-0.2, \\
\log \left(\frac{[\mathrm{N} \mathrm{II}]}{\mathrm{H} \alpha}\right)>-0.2 .
\end{array}
$$

In such a case, the left branch of BPT diagram in the left panel of Figure 3.8 would give information about the metallicity and the ionization parameter. Stasińska et al. (2006) finds that such parameters are strongly correlated, and that is what makes the left wing thin in comparison to the right wing. Therefore, $\log ([\mathrm{N} \mathrm{II}] / \mathrm{H} \alpha)$ could be used as an 


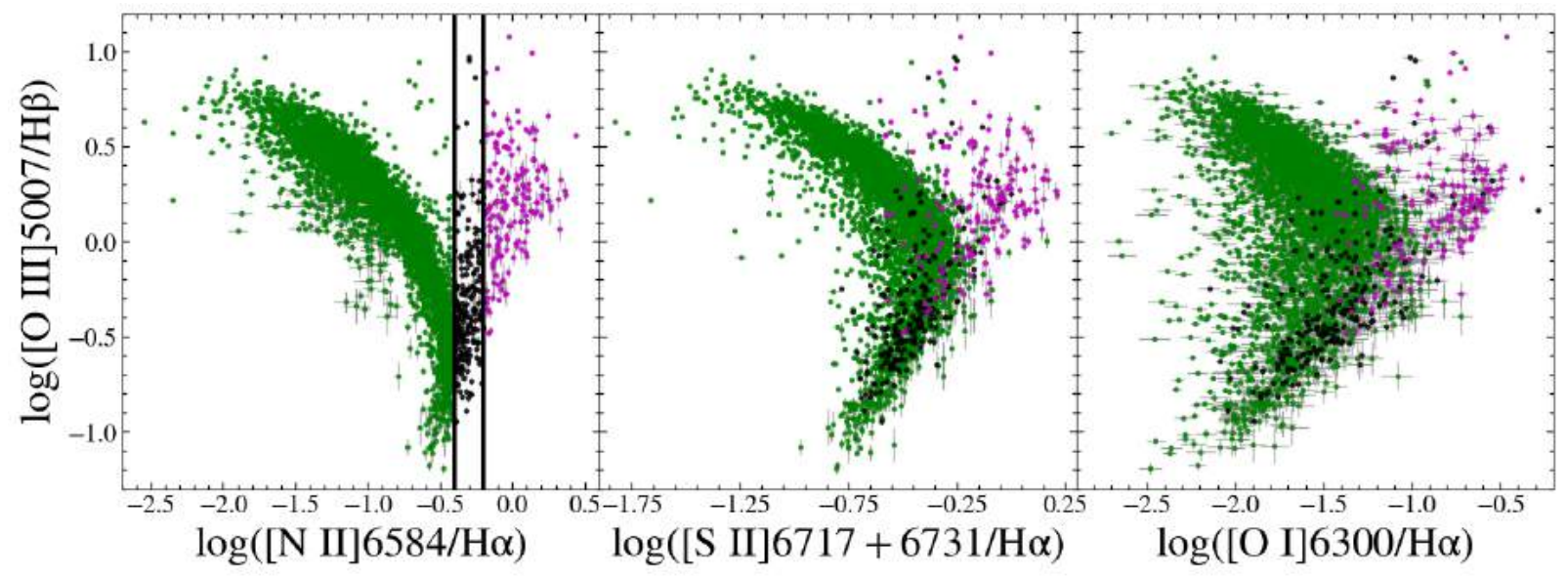

Figure 3.8: BPT (left), VO-S (middle), and VO-O (right) diagrams considering the classification scheme from Stasińska et al. (2006) (see Equation 3.12). Points in green are star-forming nuclei, in magenta are AGNs (Seyferts and LINERs), and in black are transition objects, denominated in the paper as 'hybrid'.

empirical measure of the gas metallicity, the main parameter that makes the distribution of star-forming regions a sequence. Larger values of $\log ([\mathrm{N} \mathrm{II}] / \mathrm{H} \alpha)$ would indicate that the galaxies host an AGN, with a hybrid group in the body of the seagull. As the values of $\log ([\mathrm{NII}] / \mathrm{H} \alpha)$ increase, the AGN contribution becomes larger, until the objects leave the hybrid group as they are dominated by AGN. The explanation for the right branch being fuzzier than the left one would be the dependence on other parameters that do not correlate with each other.

Due to the high level of misclassified objects seen in the middle and right panels of Figure 3.7, the classification purely based on $[\mathrm{N} \mathrm{II}] / \mathrm{H} \alpha$ is not enough to fully disentangle the body of the seagull, though it can be a solution when there are no other available line ratios to help the classification. However, some of the star-forming galaxies with low metallicity that were being misclassified as AGNs due to Equation 3.11 (positioned right at the top of the curve in the left panel of Figure 3.7) are now correctly classified, though some of the objects in this $[\mathrm{NII}] / \mathrm{H} \alpha$ range located in the upper right are probably AGNs with relatively low $[\mathrm{N} \mathrm{II}] / \mathrm{H} \alpha$ ratios.

The difficulty of finding a single parameter to classify objects according to their dominant power source was pointed out since Baldwin, Phillips \& Terlevich (1981). We are able to classify our whole sample with the criteria of Equations 3.12 , but the outliers with high values of $\log ([\mathrm{O} \mathrm{III}] / \mathrm{H} \beta)$ are misclassified as star-forming probably due to the lower metallicity of their host galaxies. The curved shape to distinguish the classes, that was a considerable gain from Kewley et al. (2001) in comparison with Ho, Filippenko \& Sargent 
(1997) and that follows the theoretical lines drawn by Veilleux and Osterbrock (1987), is clearly very important. In the cases where $\mathrm{H} \beta$ or [O III] are not available, the WHAN diagram (presented in Section 1.2.2) and one of our proposed alternative diagrams can still provide reliable classifications because it complements the $[\mathrm{N} \mathrm{II}] / \mathrm{H} \alpha$ axis with an equivalent widths or an emission-line ratio close in wavelength. Therefore, we emphasize the importance of having an axis that correlates strongly to the ionization parameter such as $\log ([\mathrm{OIII}] / \mathrm{H} \beta)$ or even $\log ([\mathrm{S} \mathrm{II}] / \mathrm{H} \alpha)$, because their combination with an axis that correlates with the metallicity such as $\log ([\mathrm{N} \mathrm{II}] / \mathrm{H} \alpha)$ gives more reliable results, even though the correlation between these two parameters is not entirely understood.

\subsubsection{Schawinski et al. 2007}

Schawinski et al. (2007) focuses on the search for signatures of AGN feedback, considering early-type galaxies from SDSS with a redshift range of $0.05<z<0.1$ (higher than that of our sample). With the aim of selecting the objects of interest, a classification scheme to distinguish Seyferts and LINERs on the BPT diagram was proposed.

Schawinski et al. (2007) finds that early-type galaxies with the higher masses present emission spectra that are mostly LINER-like. This drives the need of having a method to distinguish between bona fide LINER emission from SMBHs in a less active phase from old stellar populations with HOLMES that are also found in these old massive galaxies. For early-type galaxies with intermediate and low masses, the emission is roughly evenly distributed among star formation and AGN. Schawinski et al. (2007) also describes the behavior of these early-type galaxies in the color-mass diagram, where the star-forming galaxies are in the blue cloud, the composite objects (analogous to transition objects) in the green valley and the AGNs closer to, but not completely in, the red sequence, which is mainly composed by quiescent galaxies. By tracking the star formation history of the early-type galaxies, Schawinski et al. (2007) identifies an evolutionary sequence from star formation via nuclear activity to quiescence, plausibly related to the role of AGN feedback in quenching star formation.

Schawinski et al. (2007) applies the same S/N > 3 criteria of Kauffmann et al. (2003) to the emission lines of the BPT diagram, and considers the lines of [S II] and [O I] only when detected, since they tend to be weaker than [O III], [N II], $\mathrm{H} \alpha$, and $\mathrm{H} \beta$. The paper considers the borderlines proposed by Kewley et al. (2006) to separate star-forming objects, AGNs, 


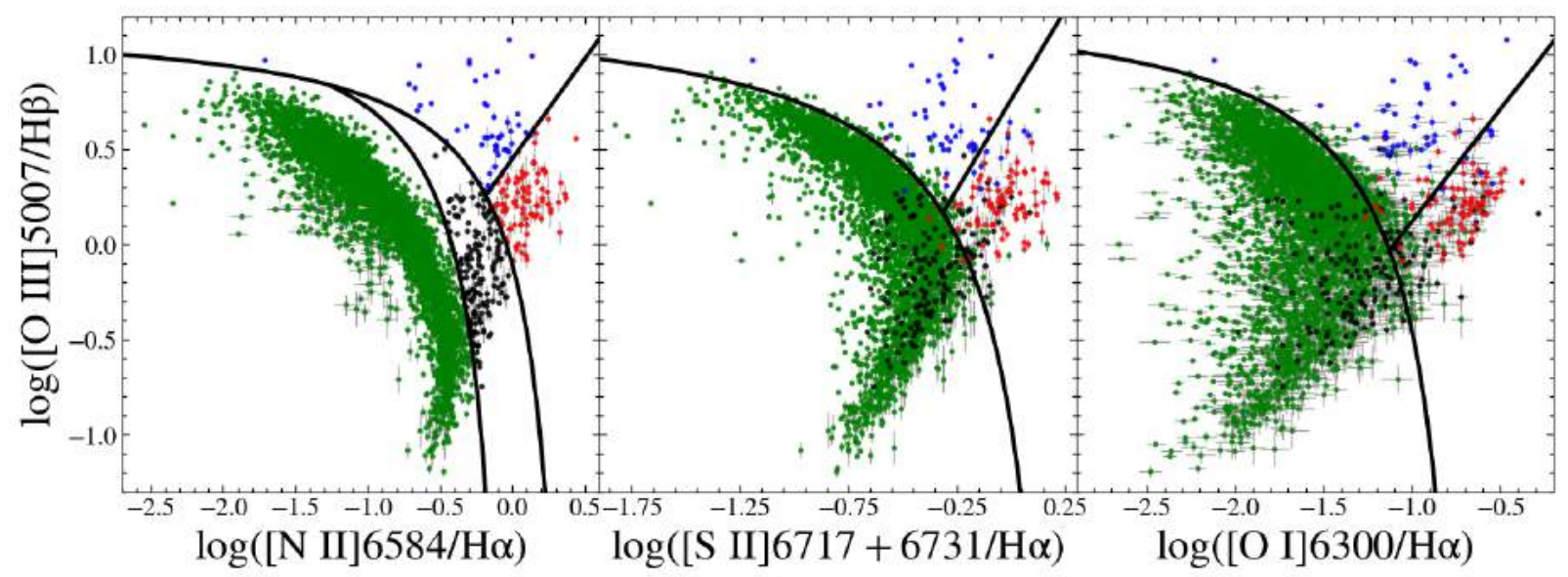

Figure 3.9: BPT (left), VO-S (middle), and VO-O (right) diagrams considering the classification scheme from Schawinski et al. (2007) and Kewley et al. (2006) (see Equations 3.1, 3.4 and 3.13). Points in green are star-forming nuclei, in red are LINERs, in blue are Seyfert nuclei, and in black are transition objects.

and composite objects.

Schawinski et al. (2007) recognizes that the best separation between the two types of AGN is achieved by using the VO-O diagrams (see the right panel of Figure 3.6), and when the $[\mathrm{OI}]$ line is too weak or unavailable, the second option should be using the VO-S diagrams (see the middle panel of Figure 3.6), considering the classification criteria of Kewley et al. (2006). However, to solve for the cases when neither [S II] nor [O I] are detected, Schawinski et al. (2007) proposes a division line for the BPT diagrams based on Kewley et al. (2006) for the VO diagrams. The Seyfert-LINER separation is proposed as a straight line (similar to Equations 3.7 to 3.10):

$$
\log \left(\frac{[\mathrm{O} \mathrm{III}]}{\mathrm{H} \beta}\right)=1.05 \log \left(\frac{[\mathrm{N} \mathrm{II}]}{\mathrm{H} \alpha}\right)+0.45 .
$$

Hence, since the new classification scheme proposed by Schawinski et al. (2007) is based on the borderlines from Kewley et al. (2006), we will consider both works to define the classes of the objects in our sample on Figure 3.9. In order to compare the ambiguous objects, we will consider the classification scheme of Kewley et al. (2006) that refers only to the BPT diagram (see the left panel of Figure 3.6), which means the lines from Kewley et al. (2001) (Equation 3.1) and Kauffmann et al. (2003) (Equation 3.4).

Though the VO borderlines will not play a role at classifying the objects in this Section, they are shown in order to help the analysis of the ambiguous objects. By considering a single diagram, we are able to classify $100 \%$ of our sample and analyze the misclassified objects in the VO diagram, stressing that in Schawinski et al. (2007) it was basically the 
other way around: the classification schemes of Kewley et al. (2006) for the VO diagrams were considered as a basis to derive the new line included in the BPT diagram.

By proposing the borderline that is able to distinguish Seyferts and LINERs on the BPT diagram on the left panel of Figure 3.9, this diagram becomes complete in separating the classes. Though Veilleux and Osterbrock (1987) and other works have pointed out that the other diagrams (VO-S and especially VO-O) are more efficient, the BPT is the most widely used diagnostic diagram because it classifies objects considering the strongest emission lines, and therefore it is the easiest to be used.

When considering the BPT borderlines of the left panel on the middle and right panels of Figure 3.9, we find a curious pattern for the AGNs. On the middle panel of VOS diagram, we see more Seyfert nuclei (blue points) in the LINER locus, while in the right panel of VO-O diagram we see more LINER-like objects (red points) in the region dominated by Seyferts. These misclassifications can arise from differences in abundance and density of objects in the same class, consistent with the fact that the AGN branch is more fuzzy suggesting that other parameters are involved besides just the metallicity and the ionization parameter.

As for the misclassified star-forming nuclei, the line proposed by Kauffmann et al. (2003), though being more restrictive than that by Kewley et al. (2001) for the maximum contribution of pure starbursts, embraces all objects clearly in the left branch. Though the lines proposed by Kewley et al. (2001) (and Kewley et al. 2006) for the VO diagrams should also represent a maximum starburst limit, there are several objects classified as starforming regions by the BPT diagram that fall just above the boundary line. Hence, maybe the borderlines from Kewley et al. (2001) (and consequently from Kewley et al. 2006) that differentiate star-forming nuclei from AGNs in the VO diagrams are too strict. Or perhaps this could indicate the need of another curve in the VO diagrams similar to the Kewley et al. (2001) and Kauffmann et al. (2003) curves in the BPT diagram, for distinguishing the composite objects in the body of the seagull. We notice that the transition objects from the BPT diagram of the left panel of Figure 3.9 are spread around in the VO diagrams of the middle and right panels, more commonly in the star-forming locus than in the LINER locus, and with some cases in the Seyfert locus. 


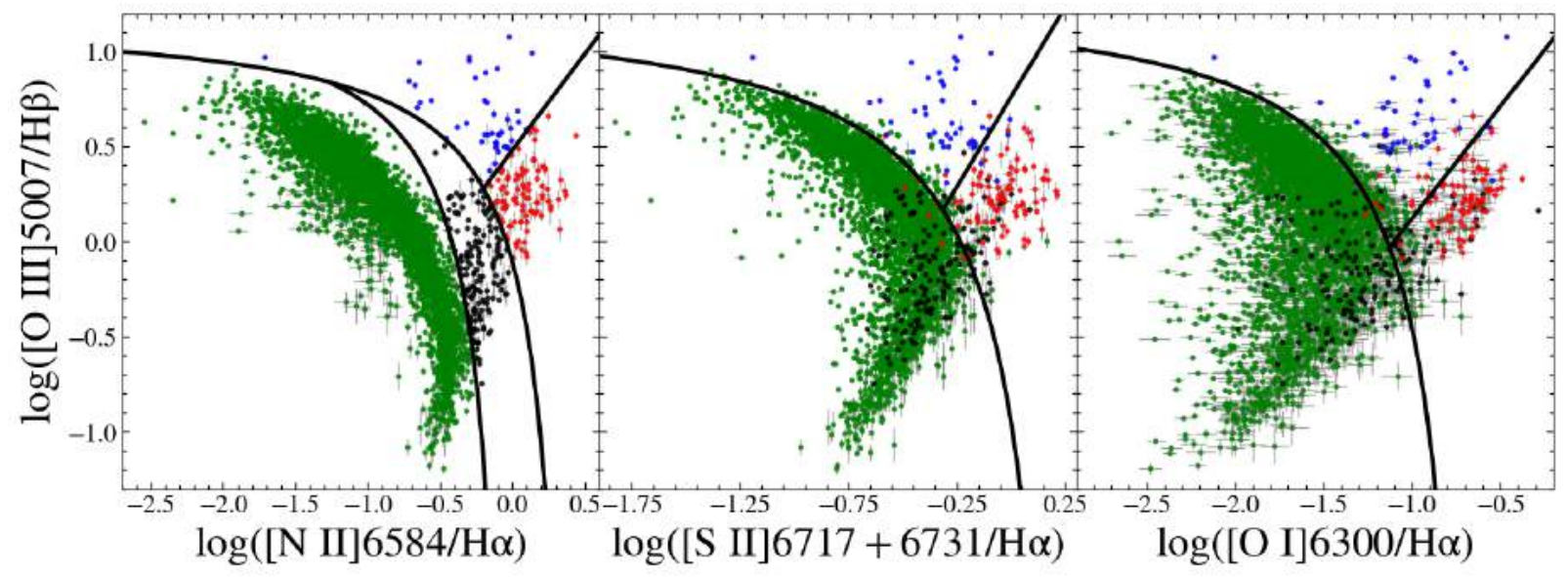

Figure 3.10: BPT (left), VO-S (middle), and VO-O (right) diagrams considering the classification scheme from Cid Fernandes et al. (2010) and Kewley et al. (2006) (see Equations 3.1, 3.4 and 3.14). Points in green are star-forming nuclei, in red are LINERs, in blue are Seyfert nuclei, and in black are transition objects.

\subsubsection{Cid Fernandes et al. 2010}

The main focus of the work of Cid Fernandes et al. (2010) is to include galaxies that show weak line ratios to have a more complete demographic census of galaxies, which is not possible with the cutoffs based on $\mathrm{S} / \mathrm{N}$ that are usually considered in the literature (see Kauffmann et al., 2003; Schawinski et al., 2007, for example). Therefore, Cid Fernandes et al. (2010) translates the Kewley et al. (2006) and Stasińska et al. (2006) lines to other diagnostic diagrams that are more economic, introduced in Section 1.2.2.

The classification scheme proposed by Cid Fernandes et al. (2010) does not bring new dividing lines, but only transpositions of the borderlines that were already proposed: the curves from Kewley et al. (2001), Kauffmann et al. (2003), and Stasińska et al. (2006) to distinguish star-forming galaxies from AGNs, and the straight lines from Kewley et al. (2006) to distinguish between Seyferts and LINERs. The method was adapted from the optimal class separation technique of Strateva et al. (2001) (see also Mateus et al., 2006). The borderline proposed to divide Seyferts and LINERs on the BPT diagram, based on the division proposed by Kewley et al. (2006), is

$$
\log \left(\frac{[\mathrm{O} \mathrm{III}]}{\mathrm{H} \beta}\right)=1.01 \log \left(\frac{[\mathrm{N} \mathrm{II}]}{\mathrm{H} \alpha}\right)+0.48 \text {. }
$$

Therefore, the classification scheme will consider the lines of Kewley et al. (2006) on the BPT diagram and the borderline proposed by Cid Fernandes et al. (2010).

Since we are considering classification based only on the BPT diagram, on the left panel 
of Figure 3.10, we are able to classify $100 \%$ of our sample and then analyze which cases are ambiguous using the VO diagrams. In the middle panel of Figure 3.10 (VO-S) there are a handful of Seyferts in the LINER region, and vice versa. The right panel of Figure 3.10 (VO-O) shows a substantial fraction of the LINERs in the Seyfert region. Hence, the overlap has a similar trend as in Schawinski et al. (2007), but with fewer ambiguous classifications. This is in agreement with Cid Fernandes et al. (2010), that mentions that the line in Equation 3.14 is similar to that of Equation 3.13, but the former is based on an optimal translation of the borderlines that were already known (from VO-S and VO-O in Equations 3.5 to 3.10 .

We again find H II region-like objects in the upper part of the star-forming sequence just above the locus of star formation. This was expected based on Kauffmann et al. (2003) and Kewley et al. (2001) because the choice of the Seyfert-LINER boundary proposed by Cid Fernandes et al. (2010) or Schawinski et al. (2007) does not affect this.

\subsubsection{Meléndez et al. 2014}

Meléndez et al. (2014) produces several detailed photoionization models of active and starburst galaxies in order to evaluate the distribution of emission-line galaxies in optical and mid-infrared diagnostic diagrams. The models are based on a two-zone approximation, which means a combination of matter- and radiation-bounded components, to optimally incorporate line emission produced under different conditions (see Appendix A for more information on this kind of model). Such models are made mainly to fit the observed midinfrared line ratios $[\mathrm{Ne} \mathrm{III}] 15.56 \mu \mathrm{m} /[\mathrm{Ne} \mathrm{II}] 12.81 \mu \mathrm{m}$ and $[\mathrm{O} \mathrm{IV}] 25.89 \mu \mathrm{m} /[\mathrm{Ne} \mathrm{III}] 15.56 \mu \mathrm{m}$, to then be compared to the line ratios of the BPT diagram and a sample of observational data from SDSS. The radiation-bounded component of the two-zone AGN approximation model with high density and low-ionization provides a good lower limit for the [N II] emission, and a theoretical demarcation line is proposed in order to determine a maximum and minimum AGN contribution.

Based on the complex models generated, Meléndez et al. (2014) proposes that an emission-line galaxy will have an AGN component if

$$
\log \left(\frac{[\mathrm{O} \mathrm{III}]}{\mathrm{H} \beta}\right)>\left(\frac{0.12}{\log ([\mathrm{N} \mathrm{II}] / \mathrm{H} \alpha)+0.34}\right)+1.15
$$

This borderline represents the lowest theoretical prediction for the AGN contribution to the 


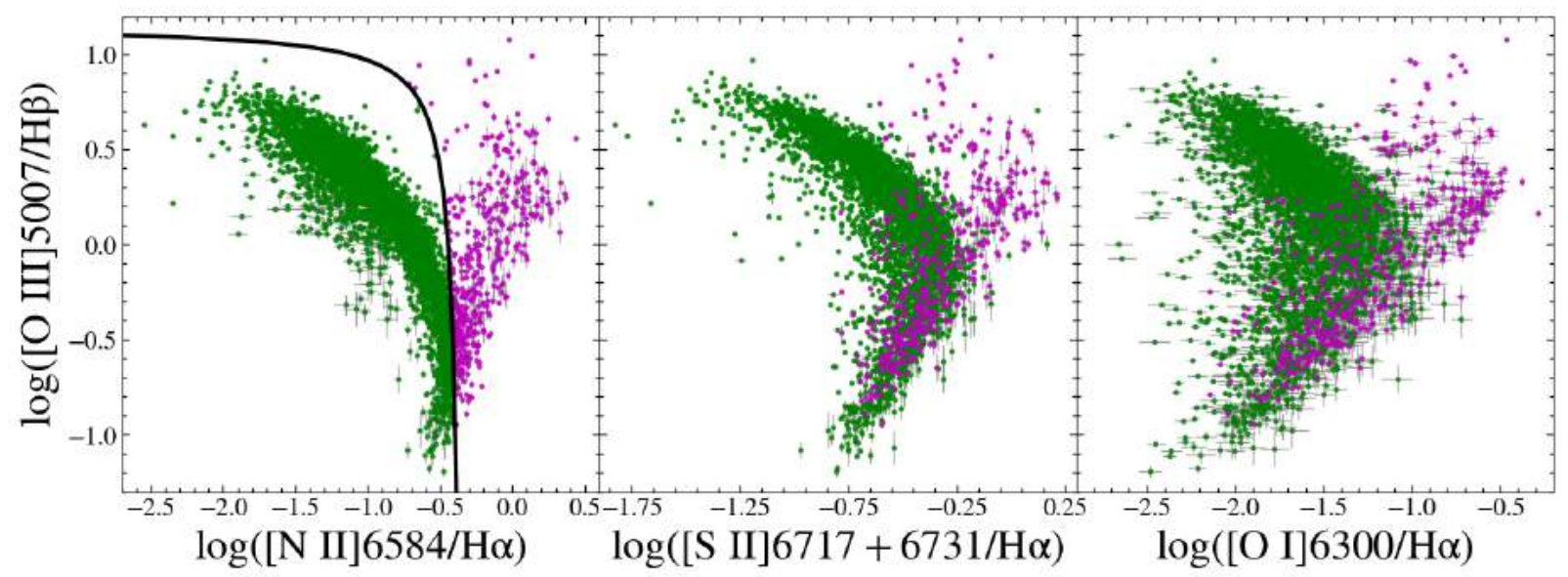

Figure 3.11: BPT (left), VO-S (middle), and VO-O (right) diagrams considering the classification scheme from Meléndez et al. (2014) (see Equation 3.15). Points in green are star-forming nuclei and in magenta are AGNs (Seyferts and LINERs).

optical spectra, and lies above the line proposed by Kauffmann et al. (2003) (see Equation 3.4. Hence, objects positioned above Equation 3.15 range from composite systems (analogous to the transition objects) to AGN dominated sources. The models from Meléndez et al. (2014) also provide a maximum AGN line, that represents the maximum contribution of AGN to a spectrum. However, it is clear from the paper that several AGNs lie above this line, so we will not consider this upper borderline in our work.

The classification scheme that divides pure star-forming sources from those that present some AGN component is applied to the diagnostic diagrams of Figure 3.11. From the large number of objects ambiguously classified in the comparison of the different panels of Figure 3.11, we can see that this classification scheme, with models based on the emission-line intensities in the mid-infrared rather than in the the optical, is not appropriate even though it is more recent than the lines proposed by Kewley et al. (2001), Kauffmann et al. (2003) and Stasińska et al. (2006). Therefore, the theoretical borderline of Equation 3.15 will not be further considered in the analysis of our sample.

\subsubsection{Discussion}

Now that the different classification schemes proposed in the literature have been presented, in this Section we will give a final discussion.

Making a chronological review, the borderlines on diagnostic diagrams were slightly altered over the years. Baldwin, Phillips \& Terlevich (1981) and Veilleux and Osterbrock (1987) were limited by the small amount of observational data and the available grids 
of photoionization models. Ho, Filippenko \& Sargent (1997) used a larger amount of uniform observational data from the Palomar Survey, establishing boundaries based on a combination of constant values for each of the axes in the traditional diagnostic diagrams. The first purely theoretical borderline was proposed by Kewley et al. (2001), which was defined by the upper envelope of the grid of photoionization models for simulated starforming regions based on ionizing sources of young star clusters. After the release of SDSS, it became feasible to propose empirical classification schemes, since that survey offered a large amount of uniform data on nearby galaxies and advanced starlight subtraction techniques to obtain the pure nebular spectra. Hence, with the shape of the seagull visible by the thousands of objects assembled, Kauffmann et al. (2003) proposed an empirical boundary to the BPT diagram significantly below the theoretical line proposed by Kewley et al. (2001). Then, Stasińska et al. (2006) proposed another boundary to divide starforming objects from AGN, based on both observational data from SDSS and theoretical photoionization models, and later that year Kewley et al. (2006) proposed a scheme that united Kewley et al. (2001) and Kauffmann et al. (2003) by proposing additional lines in the VO diagrams to separate Seyferts and LINERs. In order to define borderlines that distinguish the two types of AGN in the BPT diagram, Schawinski et al. (2007) and Cid Fernandes et al. (2010) proposed a straight line based on Kewley et al. (2006) for the VO diagrams, with an empirical and an optimal transposition approach, respectively. Finally, we mentioned the work of Meléndez et al. (2014) whose theoretical boundaries for AGN aimed to fit mid-infrared lines more than optical emission lines. These showed too many ambiguous classifications in comparison with the previous works.

Table 3.1.1.9 shows the percentages of the classes of galactic nuclei for the classification schemes that were analyzed. The second column, 'Total', indicates the amount of objects from our sample that were classified according to each of the schemes. The classification schemes proposed by Kauffmann et al. (2003), Schawinski et al. (2007), Cid Fernandes et al. (2010), and Meléndez et al. (2014) consider only the BPT diagram, and therefore can classify the whole sample. The two classification schemes proposed by Stasińska et al. (2006) also consider only the BPT diagram, but with restrictions regarding the values where their scheme is valid, in a way that not all the objects from our sample are being classified. The cases where more than one diagram is being considered for the classification of objects indicate the percentage of ambiguous classifications: $15.62 \%$ for the Ho, Filip- 
Table 3.3 - Results of the classification schemes of Section 3.1.1 applied to our sample. The first column gives the paper where the classification scheme was presented: Ho97 for Ho, Filippenko \& Sargent (1997) (Figure 3.3), Ke01 for Kewley et al. (2001) (Figure 3.4), Ka03 for Kauffmann et al. (2003) (Figure 3.5), Ke06 for Kewley et al. (2006) (Figure 3.6), St06 and St06-N for Stasińska et al. (2006) (Figures 3.7 and 3.8. respectively), Sc07 for Schawinski et al. (2007) (Figure 3.9), CF10 for Cid Fernandes et al. (2010) (Figure 3.10, and Me14 for Meléndez et al. (2014) (Figure 3.11). The column 'Total' indicates the percentage of our total sample that is being classified according to the scheme. The column 'SF' indicates the percentage of objects that are classified as star-forming nuclei. The column 'Transition' indicates the percentage of transition objects. The column 'AGN' indicates the percentage of AGNs. If the scheme classifies objects as Seyferts and LINERs, their percentages are shown in the last two columns, and the 'AGN' column is the sum of both the 'Seyfert' and 'LINER' columns.

\begin{tabular}{|l|l|l|l|l|l|l|}
\hline Classification & Total(\%) & SF $(\%)$ & Transition(\%) & AGN(\%) & Seyfert(\%) & LINER(\%) \\
\hline Ho97 & 84.38 & 96.52 & 1.19 & 2.29 & 0.59 & 1.7 \\
\hline Ke01 & 92.82 & 97.2 & - & 2.8 & - & - \\
\hline Ka03 & 100 & 93.2 & - & 6.8 & - & - \\
\hline Ke06 & 96.02 & 91.25 & 3.83 & 4.92 & 2.73 & 2.19 \\
\hline St06 & 99.26 & 89.33 & - & 10.67 & - & - \\
\hline St06-N & 100 & 91.03 & 5.13 & 3.84 & - & - \\
\hline Sc07 & 100 & 93.11 & 3.67 & 3.22 & 1.19 & 2.03 \\
\hline CF10 & 100 & 93.11 & 3.67 & 3.22 & 1.05 & 2.17 \\
\hline Me14 & 100 & 89.86 & - & 10.14 & - & - \\
\hline
\end{tabular}

penko \& Sargent (1997) scheme, 7.18\% for the Kewley et al. (2001) scheme, and 3.98\% for the Kewley et al. (2006) scheme (the inclusion of more objects in this case is probably associated to the transition objects, in comparison to Kewley et al. 2001). The sum of the 'SF', 'Transition', and 'AGN' columns is of 100\%, referring to the 'Total' that is being analyzed and not to the whole sample in the cases where such 'Total' is not of $100 \%$. The 'AGN' column indicates the sum of the columns 'Seyfert' and 'LINER' in the cases where such types of objects are considered. The two classification schemes where we have more than $10 \%$ of the objects being classified as 'AGN' are the ones proposed by Stasińska et al. (2006) and Meléndez et al. (2014), but we find such schemes too strict to the star-forming population that dominates the classification scheme with more than $90 \%$ of the objects according to all the remaining classification schemes. The schemes proposed by Schawinski et al. (2007) and Cid Fernandes et al. (2010) differ only in the distribution of Seyferts and LINERs, as expected, since both approaches consider the remaining boundary lines from the classification suggested by Kewley et al. (2006) for the BPT diagram only. We emphasize that these distributions must not be expanded to nuclei of galaxies in general, since 
our sample is biased for objects with strong emission and does not represent a demographic census of the whole population of galactic nuclei.

It is worth pointing out that not all the objects that lie below maximum starburst lines as proposed by Kewley et al. (2001) and Stasińska et al. (2006) or empirical starforming sequence line as proposed by Kauffmann et al. (2003) are necessarily photoionized by massive young stars. As shown by Espinosa-Ponce et al. (2020), the diffuse ionized gas produced by hot old stars can also populate this region. Also, objects with pure low-velocity shocks can fall below these borderlines, as shown by López-Cobá et al. (2019).

With the advent of large surveys and their contribution to the demographic study of galaxies, we were able to recognize the main patterns of emission-line objects in the diagnostic diagrams. In the VO-S and VO-O diagrams, it is easier to distinguish two regions on the right branch with Seyferts more to the top and LINERs more to the bottom. Though such objects overlap more in the BPT diagram, it is still possible to define a dividing line here. The fact that no set of boundary lines are devoid of ambiguous classifications reflects the intrinsic complexity of AGNs. Therefore, though the empirically motivated boundaries are necessarily subject to selection effects, once they have passed through consistency checks based on other observational studies, these borderlines show fewer misclassified objects than the purely theoretical lines. Though the synthetic models provide important insights into the ranges of the physical parameters that are relevant, the models also show several limitations, as discussed in more detail in Section 3.3. Hence, the best option would be to combine theoretical models and observational data to propose the classification scheme, considering the limitations and caveats of both approaches.

In our analysis we will consider all the objects in our sample and highlight any misclassification when plotted in alternative diagnostic diagrams. Therefore, we have chosen to maintain the criteria based solely on the most commonly adopted BPT diagram. In view of that, and also to evaluate the transition objects, we will adopt the lines from Kewley et al. (2001) and Kauffmann et al. (2003), highlighting the star-forming based on the latter and the discrepancy with the purely theoretical borderline of the former. To divide the AGN from our sample between Seyferts and LINERs, we have chosen the classification scheme proposed by Cid Fernandes et al. (2010), which has fewer cases of ambiguous classifications for our sample than Schawinski et al. (2007). Therefore, the main classification scheme adopted in this work is the one from Figure 3.10 unless otherwise stated. 
In Section 3.1.2, we will discuss the transition objects and the common misinterpretation of simplistic boundary lines that do not necessarily reflect the complexity of galactic nuclei. Last, we note that the ambiguous classification of some objects might be already explicit by the error bars at a single diagram. However, in our sample where the uncertainties are accounted for, the classification discrepancies may be more related to the true complexity of the objects or to the difficulties in matching different diagnostic diagrams. This issue will be discussed in Section 3.1.3.

\subsubsection{Transition objects}

The objects that do not show a typical spectra of a gaseous nebula that is photoionized either by hot stars nor by an AGN have been classified in several ways throughout the years: transition objects, composite objects, hybrid objects, and so on. The region they occupy in the BPT diagnostic diagrams, between the lines proposed by Kewley et al. (2001) and Kauffmann et al. (2003) (see Equations 3.1 and 3.4 and the transition region in Figure 3.6 is also denominated differently depending on the work that mentions it, being the transition region, the intermediate region, the mixing sequence, etc.. Such distinct denominations already point out to the polemics that such objects raise in the taxonomy of galactic nuclei.

These objects with features of both stellar and nuclear photoionization in their spectra have been associated with low resolution of the data since the standard diagnostic diagrams were proposed. Veilleux and Osterbrock (1987) argues that the difficulty in setting boundaries to divide narrow-line AGNs and H II region-like objects, considering the uncertainties of the emission-line intensity ratios and of the position of the borderlines, makes the nature of any object that falls within the transition zones to be very hard to determine. According to such work, these objects can be either pure star-forming regions, or pure narrow-line AGNs that have been shifted toward the boundaries due to errors in the line ratios, or objects with the presence of both types of excitation mechanism Binette, 1985), or even objects with a central power-law source but surrounded by stellar activity, in a way that the outskirts of the nuclei are dominated by ionization from hot stars. Ho et al. (1993) proposes that the transition objects with nuclear emission-line properties intermediate between those of AGNs and of star-forming regions would be explained by "normal" LINERs whose integrated spectra is being diluted or contaminated by neighboring H II re- 
gions, reinforcing the idea of composite systems with both kinds of activity. This is one of the arguments that lead Ho, Filippenko \& Sargent (1997) to set a transition region in the VO-O diagram, where the dependence on the ionization parameter is clearer and the LINERs can be more isolated from Seyfert galaxies (see the right panel from Figure 3.3).

Addressing this issue theoretically is not trivial, since photoionization models that consider a pure AGN (e.g., Ferland and Netzer, 1983; Halpern and Steiner, 1983; Stasińska, 1984), or a purely old stellar population (Stasińska et al., 2008), or even the ones that consider a young metal-rich stellar population with high density and low ionization parameter (Filippenko and Terlevich, 1992; Shields, 1992; Kewley et al., 2001) are all able to populate the transition region between the two branches of the BPT diagram. The situation gets even more confusing when shock models can be added to the photoionization models, recalling that shocks can derive from stellar winds (showing lower velocities) or from AGN outflows and jets (showing higher velocities). Kauffmann and Heckman (2009) proposed models with different percentages of the star-forming and the AGN contributions to show that these can also populate the transition region, with models that have more influence of the star-forming processes being positioned more to the left and the ones with higher fraction of AGN contribution positioned more to the right of the BPT diagram, as expected. One of the interpretations of such models is that there is no physical sharp boundary to divide AGN and star-forming galaxies, with a smooth variation between the classes, because galactic nuclei with lower black hole activity and ongoing star formation are observed, and hence this dubious dominance between the two ionizing mechanisms can indeed occur in nature. Kauffmann and Heckman (2009) define that the objects in the transition region between Kewley et al. (2001) and Kauffmann et al. (2003) lines present values for [O III] luminosity that would be explained by the contribution of $\sim 40-50 \%$ from star-forming processes, while the objects above the line proposed by Kewley et al. (2001) would be best modeled by objects with a contribution of $\sim 10-20 \%$ from star formation. However, though this models are useful to better understand the statistical distribution of the objects, it will not be accurate to describe individual objects in the transition region. Hence, it is important to search for observational information to help disentangling these issues.

The alternative diagnostic diagrams can help on the analysis of the different loci that the transition objects might occupy, stressing that probably not all the objects in the 
transition region will have the same ionization mechanisms. In the diagnostic diagram that considers $[\mathrm{O}$ II $] \lambda \lambda 3726,3729$ emission line, for example, the transition objects of the sample used in Kewley et al. (2006) lie within the star-forming sequence, and that also indicates that the emission of ionizing photons in such objects is likely to be dominated by their star formation. More examples considering the emission lines of the standard diagnostic diagrams will be discussed in Section 3.2 .

When Schawinski et al. (2007) analyzes early-type galaxies with regard to the evolution between star formation and quiescence, the transition objects overlap with the characteristics of Seyfert galaxies. According to such work, this suggests that the objects in this moment of the evolution time sequence might be switching back and forth between the transition phase (which would be a composite phase of AGN and star formation) and a Seyfert phase (pure AGN). Such effect would be associated also to the positive and negative AGN feedback cycles, that can alter the prevalence of star formation with time, depending on the AGN processes. The positive feedback is associated with the accumulation of gas that could form stars due to jets and outflows, while the negative feedback is associated with the expulsion of such gas in a later moment (Silk and Rees, 1998; Silk, 2005). If this is the case, then the Seyfert phase would represent an episode of enhanced accretion of the $\mathrm{SMBH}$, with low residual star formation, while the transition phase would be a moment in the nuclear evolution where both kinds of photoionization are intense. However, Schawinski et al. (2007) argues that this is not the explanation for all the transition objects in the considered early-type galaxies sample. Some transition objects are found in very massive galaxies with no evidence of star formation, in a way that they would not be part of the time sequence between stellar and nuclear activity explained above. Their properties are too similar to those of high-mass LINERs, which leads to the conclusion that the extreme starburst line proposed by Kewley et al. (2001) give onto the misclassification of some LINERs as transition objects, according to what Ho et al. (1993) pointed as an issue before the theoretical borderline was proposed.

There are several critics with regard to the borderline introduced by Kewley et al. (2001), mainly pointing to the naive usage of such theoretical boundary. Many works consider in a simplistic fashion that all the objects that lie below the borderline from Kauffmann et al. (2003) (Equation 3.4) are pure star-forming objects, with all the galaxies above the Kewley et al. (2001) line (Equation 3.1) being pure AGN and the nuclei that 
reside in between such division lines being classified as composite. Nevertheless, since the line from Kewley et al. (2001) is derived from stellar photoionization models, the objects that appear in the transition region can be explained theoretically without the presence of an AGN, and the objects above such line could represent objects with activity from both stellar and nuclear sources. The purpose of this line was never to delimit the region of pure AGN, as it is often naively used in the literature. On the other way around, the Kewley et al. (2001) line proposes a lower boundary to what could be composite objects (Cid Fernandes et al. 2010). Therefore, in order to understand where the points that shape the body of the seagull in the BPT diagram will be placed at the alternative diagnostic diagrams that will be proposed, we decided to maintain this region between Kewley et al. (2001) and Kauffmann et al. (2003) as a separate class, that we will refer to as transition objects, since their nature as composed objects is rather controversial. Another possible issue with regard to Kewley et al. (2001) division line is that the stellar population synthesis considered in the theoretical models that based such classification scheme considers planetary nebula nuclei atmosphere models to synthesize the Wolf-Rayet stars, and these atmospheres produce spectra that are harder than the typical Wolf-Rayet atmosphere models (D'Agostino et al., 2019). Therefore, these most extreme cases might not be realistic, and that emphasizes the fact that the upper limit of star formation can be overestimated by the Kewley et al. (2001) line.

Some authors argue that this issue in the classification of spectra that contain signatures of both types of ionization is due to the low spatial resolution of spectra observed with finite-size aperture slits, since they will sum the contributions of the areas of the observed object that fit inside the observed region. In that way, the blending of the emission lines produced by the AGN and by the H II regions would be more serious for more distant and thus angularly smaller galaxies (Veilleux and Osterbrock, 1987). However, more recent works have shown that the fixed aperture-size does not affect the parameters derived for the SDSS sample for redshift $z<0.4$ (Dors et al., 2020). We have checked the dependence of the emission line ratios of the BPT diagnostic diagram with regard to the redshift and also the distributions of the amount of objects of each class according to redshift, and we find no significant trend or correlation that could be associated with higher contamination for the higher redshift values in our range, for any of the classes (see Appendix B.1). However, we emphasize that this is probably associated to the small redshift cut we are 
considering, with nearby objects at $z<0.016$.

Nevertheless, the study of galactic nuclei with integral field units (IFU) is revealing that, when each spaxel of a single galaxy that is being observed as a data cube has its spectrum plotted in the BPT diagnostic diagram, for the objects that show both nuclear and stellar activities, there will be some spaxels that will fall in the transition region (D'Agostino et al., 2019: Sánchez, 2020). For this type of object, usually the spread of the spaxel's emissionline ratios is continuous from the star-forming region of the BPT diagram towards the AGN classification region, which suggests that this mixing between star-forming and AGN features happens even when spatially resolved spectra are provided. If the presence of shocks influences such continuous behavior, than the ratio of the intensities of emission lines should be reconsidered with regard to AGN contribution, because if the shocks are that relevant, then the AGN emission is likely being overestimated (D'Agostino et al. 2019).

On the work from da Silva (2020), IFU data of galaxies morphologically similar to the Milky Way at the Southern Hemisphere are analyzed in the BPT diagrams. The comparison is made between an analogous subsample from the Palomar Survey (Northern Hemisphere, using slits), the nuclear regions of the galaxies, and the circumnuclear regions of the galaxies. This separation between the regions is only feasible due to the spatial resolution of the data cubes. The results of such comparison indicate that the behaviour of the circumnuclear regions of the IFU galaxies is similar to the distribution of the Palomar galaxies, while in the plot with solely the nuclear regions none of the 10 analyzed galaxies reside in the transition region of the BPT diagram. Thus, this indicates that the integrated spectra obtained from slits (the case of SDSS would be analogous to the Palomar Survey, notwithstanding the fact that the Palomar Survey is focused on the nuclear regions of galaxies, while SDSS uses a fixed-size aperture to obtain the spectra of thousands of galaxies at different distances) is more similar to the information of the circumnuclear region of the galaxies than the nucleus itself, which means they are associated with larger areas of the galactic centers. In this work of da Silva (2020), some of the examples of galaxies in which the circumnuclear regions fall in the transition region, when observed in detail with spatial resolution, show that they host a LINER nuclei and some separate though very close H II regions. Further analysis are being provided by the DIVING3D Survey (associated with the work of da Silva 2020) to have more examples of the contamination 
by the HII regions in the outskirts of the nuclei being responsible for shifting the AGNs to the transition region in some of the cases. Anyway, data with higher spatial resolution seems to be the bet for disentangling the transition object issue (Menezes et al., 2020).

Overall, the physical explanation for transition nuclei remains an unresolved problem because basically all the solutions are applicable: pure star formation, old stellar populations, pure AGN, shock contributions, and any combination of the mentioned ionization mechanisms. We believe that the objects that lie in the transition region of the BPT diagram are not explained solely by one of the options above. We have chosen to maintain the objects between Kewley et al. (2001) and Kauffmann et al. (2003) borderlines in the BPT diagram being classified as transition objects in order to evaluate the behaviour of such controversial objects in the alternative diagnostic diagrams that will be proposed.

\subsubsection{Misclassification}

The main issue in the use of distinct diagnostic diagrams, which is the ambiguous classification of the same object, is also a source of important insights with regard to the nature of galactic nuclei. After all, when an object is classified differently depending on the considered axes, this indicates us that something relevant might be happening in that object, or that its spectrum might present limitations, which are both important concerns to be better understood.

Ho, Filippenko \& Sargent (1997) already pointed out to the possible ambiguity as the main problem of considering three different diagrams to classify objects. This issue can be related to the fact that the conditions that involve the low ionization forbidden lines of the different abscissas do not always hold simultaneously. There could be, for example, selective abundance variations that could cause a diagnostic to be different for the same object being considered in a distinct diagram, and that only emphasises the empirical origin of the standard diagnostic diagrams. Another possible explanation for the ambiguity is the substantial uncertainty that can be associated with any given lineintensity ratio. Ho, Filippenko \& Sargent (1997) suggests that, in order to give more weight to one or another diagram, the [O I] should be preferred if the line is available (it is the most sensitive diagram to the shape of the ionizing spectrum) and [N II] should be avoided (due to potential selective enhancement of nitrogen in galactic nuclei, see e.g., Storchi-Bergmann and Pastoriza, 1990). However, the most common use in the literature 
nowadays is to consider only the BPT diagram, since the [N II] tends to be stronger than [S II] and mainly [O I].

In this Section, we will discuss several possibilities that have been pointed out to justify the misclassification of star-forming regions as AGNs and vice versa. Here, we are going to introduce some new actors to the play which might be contributing to the intensity of the emission lines, such as shocks, diffuse ionized gas (DIG), and old stellar populations. Also, we will discuss some technical limitations that might reflect in the uncertainties of the emission-line ratios, like the aperture size of the telescopes being used and the difficulty in measuring faint lines.

First, we will refer to star-forming objects that can be classified in the AGN loci. Then, we will talk about AGNs that can be misclassified as star-forming regions and also about several AGNs that cannot be placed in the standard diagnostic diagrams. After that, we will discuss the difficulty in separating LINERs and extended LINER-like emission (which is often associated with old stellar populations) when using the line ratios of the traditional diagnostic diagrams. And at last, we will mention the DIG and the shocks, that might contribute to the emission-line intensities in all of the classes.

Though Kewley et al. (2001) proposed a theoretical maximum starburst line, there are some cases where the star-forming regions classified according to BPT diagrams fall in the AGN locus for the VO diagrams (see Figure 3.10 for examples) and also some few rare cases of star-forming galaxies that lie in the AGN locus on the BPT diagram. The later cases have been observed as very young starburst galaxies that probably do not host an AGN, and are associated with high ionization parameter and spectral hardness (e.g., Overzier et al., 2009; Loaiza-Agudelo et al., 2020). Nevertheless, star-forming galaxies above the Kewley et al. (2001) line can also be modeled if we consider high densities and metallicities (Feltre et al., 2016).

However, there are two parameters that help us to trace ongoing star formation, and though they are not in the diagnostic diagram's axes, they can be evaluated for the few cases of misclassification. Such parameters are the equivalent width of $\mathrm{H} \alpha(\mathrm{EW}(\mathrm{H} \alpha))$ and the break at $4000 \AA\left(\mathrm{D}_{\mathrm{N}}(4000)\right)$ Balogh et al., 1999), already explored by Stasińska et al. (2008) and Cid Fernandes et al. (2011) to propose alternative diagnostic diagrams that can distinguish young from old stellar populations (see Section 1.2.2). $\mathrm{D}_{\mathrm{N}}(4000)$ is a proxy for the light weighted mean stellar age, being sensitive to small levels of star formation 
(Yan and Blanton, 2012). The observed break is a consequence of the combination of the Balmer break with a number of metal lines in a narrow wavelength region. For hot stars, these metals are multiply ionized and the resulting break will be small, dominated by the Balmer break. Along the stellar cluster evolution, the stellar population becomes dominated by cooler stars, the break increases due to metal-line blanketing (Belfiore et al. 2016). $\mathrm{EW}(\mathrm{H} \alpha)$ shows a bimodal behavior with the empirical discrimination at $\mathrm{EW}(\mathrm{H} \alpha) \sim$ $3 \AA$, that is one of the classification schemes of the WHAN diagram Cid Fernandes et al. 2011). Despite the difficulty in modeling old stellar populations and the pAGB evolution, the threshold of $\mathrm{EW}(\mathrm{H} \alpha) \lesssim 3 \AA$ is in agreement with the expectations from reprocessing of the ionizing radiation from hot evolved stars (Binette et al., 1994b; Stasińska et al., 2008; Cid Fernandes et al., 2011), while star-forming objects and AGNs will both present higher values for $\mathrm{EW}(\mathrm{H} \alpha)$ (Belfiore et al., 2016). Other recombination lines of hydrogen can be considered in this comparison, depending on the available spectral coverage.

Belfiore et al. (2016) compared single fibre SDSS spectra with spatially resolved MaNGA spectra and found that young stellar populations (presenting low $\mathrm{D}_{\mathrm{N}}(4000)$ and high $\mathrm{EW}(\mathrm{H} \delta))$ of the data, with higher spatial resolution, reach higher values of the line ratios $[\mathrm{S} \mathrm{II}] / \mathrm{H} \alpha,[\mathrm{N} \mathrm{II}] / \mathrm{H} \alpha$, and $[\mathrm{O} \mathrm{III}] / \mathrm{H} \beta$, above the demarcation line set as a maximum starburst boundary by Kewley et al. (2001), particularly to the VO-S diagram (they do not consider the VO-O diagram), in agreement to what our sample shows even without the spatial resolution of integral field spectroscopic data. Belfiore et al. (2016) justifies that this effect is more severe to the $[\mathrm{S} \mathrm{II}] / \mathrm{H} \alpha$ line ratio because $[\mathrm{S} \mathrm{II}]$ is a low-ionization line emitted mainly in the partially ionized region of the nebula, in a way that it is more effective for distinguishing $\mathrm{H}$ II region-like and LINER-like ionization than the $[\mathrm{N} I \mathrm{I}] / \mathrm{H} \alpha$ line ratio, in agreement with other precursor works as Veilleux and Osterbrock (1987) and Ho et al. (1997).

As to misclassified AGNs, Agostino and Salim (2019) point out to the main causes of such occurrence being associated with: star-formation dilution, broad line contamination, AGNs without emission lines, and too low ionization level AGNs.

Regarding the star formation dilution, this problem with the contamination of starlight to the spectrum of an AGN occurs often when integrated spectra from slits are being used. The spectroscopic fibers that gather all the information of a limited-size region will include the emission from the nucleus and also from $\mathrm{H}$ II regions that might be neighbouring the 
nucleus, affecting the resulting line ratios in a way that a bona fide AGN can be classified as a transition object or a star-forming nuclei, though the central ionization source is associated with SMBH activity. However, though Moran et al. (2002) shows that narrowline AGNs may be misclassificed as star-forming nuclei, if the spectroscopic aperture is large enough and the starlight contamination occurs, only the aperture effect cannot explain the optical misclassification, since several correctly classified AGNs show high specific star formation rates and there is no correlation between the misclassification and the redshifts and sizes of the galaxies. Therefore, though the high star-formation rate is associated with the AGNs that are misclassified as star-forming regions, this issue might be more related to the dominance of the process, with the missclassified AGNs presenting lower luminosities or lower accretion rates, and thus being more easily impacted by the surrounding star formation (Agostino and Salim, 2019).

As for the broad-line contamination, the classifications might be incorrect if the AGN shows relatively broad Balmer lines, in a way that they are not large enough to be considered type 1 AGN, but they are broad enough to affect the measured fluxes of the hydrogen lines and consequently the emission-line ratios will shift the AGN to the star-forming region of the diagnostic diagrams. Specially for the MPA-JHU catalog, that is considered for our sample, it should be noted that their emission-line properties are derived using single Gaussian fits, which might underestimate the FWHM of certain lines that present weak broad wings (Agostino and Salim, 2019).

Another issue to be considered is the absence of emission lines in many detected AGNs. This can be due to dust obscuration of X-ray detected AGNs, in a way that they emit the lines but they are heavily attenuated inside the host galaxy (Rigby et al., 2006; Goulding and Alexander, 2009; Trump et al., 2009). Depending on the X-ray measured luminosity of the AGN, its expected optical emission will be insufficient to be detected (Yan et al. 2011). Another option is associated with the intrinsic absence of the emission lines, though the AGN is present. This can be due to the AGN being in a less active phase, and thus presenting a radiativelly inefficient accretion flow that is not strong enough to heat the NLR adequately (Yuan and Narayan, 2004; Trump et al., 2009). Other reason for this intrinsic absence would be the NRL not being sufficiently heated because of its complex structure, that could allow many of the ionizing photons to escape (Trouille and Barger, 2010). Finally, the AGN could have only recently been activated, in a way that there was 
not enough time for its activity to heat the nuclear region for the emission lines to be produced (Schawinski et al., 2015). In these cases where the lines are not being produced, if the host galaxies contain H II regions in their nuclei, they would be correctly classified as star-forming objects by the diagnostic diagrams, since this would be the dominant source of the detected emission lines, though there might be some low level AGN activity happening. Agostino and Salim (2019) analyzes that intrinsically weak and/or non-detectable NLR are the most likely explanations for the majority of cases of AGNs that are detected in X-Rays and do not reside in the AGN locus of the diagnostic diagrams, and once these objects would not be classifiable as AGN even if there was no ongoing star-formation, it is not appropriate to refer to them as misclassified.

Therefore, the absence of emission lines, due to obscuration by dust or to an environment that has not been heated enough to produce the lines, points out to intrinsic difficulties in placing the AGNs in the emission-line ratio diagnostic diagrams, independently on the data quality. That leaves a considerable amount of AGNs $(\sim 40 \%$ of the X-ray AGN sample of Agostino and Salim 2019) to be unclassifiable by the diagnostic diagrams - more than simply missclassified - emphasizing that only a part of the AGN population can be applied to such classification method. Nevertheless, it is important to consider such cases (NLRs heavily obscured by dust, low-ionization incapable of sufficiently heating the NLR to produce the emission lines, or early stages of the cycle) in the wide covering of the AGN population.

The final reason for missclassified AGNs can be related to a very low ionization level, in a way that the emission lines would be produced and associated with the AGNs, but they would be so dim that the object would be placed in the star-forming branch of the diagnostic diagrams (Agostino and Salim, 2019).

Now we will go into further details about the LINER-like emission that can be found outside the nuclear regions of galaxies and that consequently are not associated with the activity of a central SMBH, but for being observed, must be contributing to the intensity of the measured lines in several cases. LINERs are associated with a radially flat $\mathrm{EW}(\mathrm{H} \alpha)$ profile, with values of $\mathrm{EW}(\mathrm{H} \alpha<3 \AA)$, associated with the lack of young stellar populations as mentioned above, which implies that the line emission follows tightly the continuum due to the underlying old stellar population (Belfiore et al., 2016). These objects are identified by their strong low-ionization forbidden lines (as [N II], [S II], and [O I]) relative 
to recombination lines $(\mathrm{H} \alpha, \mathrm{H} \beta)$ and to high-ionization forbidden lines (as [O III]).

However, this particular pattern of the intensity of the emission lines is not exclusive of a single astrophysical event, in a way that the LINER-like emission can be associated with photoionization by a low-ionization AGN (e.g., Ferland and Netzer, 1983; Halpern and Steiner, 1983; Groves et al., 2004), with photoionization by HOLMES (e.g., Stasińska et al., 2008; Cid Fernandes et al., 2011), with fast radiative shocks (e.g., Dopita and Sutherland, 1995, 1996), with photoionization by the hot X-ray-emitting gas (e.g., Voit and Donahue, 1990; Donahue and Voit, 1991), or with thermal conduction from hot gas (e.g., Sparks et al., 1989). We recall here that LINER stands for 'low-ionization nuclear emission-line regions', as defined by Heckman (1980), and people suggested the term LIER to the warm ionized gas that emits similarly to LINERs without being in the nuclei of galaxies (e.g., Belfiore et al., 2016). We will not adopt such terminology because, though it is a nice pun about the misclassification going on in the diagnostic diagrams, we prefer to try to classify the objects by what they are, and not by what they are not. Therefore, we will refer to this emission as LINER-like and try to determine the cases when it is associated with HOLMES (see Section 1.1.3.3), to DIG, to shocks, or to other possible ionization mechanisms that reproduce a LINER-like spectrum.

Though Yan and Blanton (2012) work with low spatial resolution data, their main conclusion is that the line emission in most of the galaxies classified as LINERs through the standard diagnostic diagrams, considering data obtained by large aperture spectroscopy, is not primarily powered by AGN activity, and, therefore, the emission lines themselves do not trace the AGN bolometric luminosity. One of the results that lead to this conclusion is that most early-type galaxies that have nuclear emission line regions also have extended line-emitting regions, and hence, an integrated spectrum is considering the contribution of whatever is inside the aperture size. This consideration of the extended emitting regions affecting the measured intensity of the emission lines is made for apertures that gather an area larger than 100 pc. Even though we have tried to confine our sample to the nuclear regions of nearby galaxies, since the biggest areas considered in our sample (on the limit of $z=0.016$ ) integrate a circle with diameter of almost $1 \mathrm{kpc}$, the contamination from outer and extended regions might be relevant for our low-redshift sample, due to the fixed aperture size of SDSS. Based on the scales defined by Yan and Blanton (2012) to distinguish nuclear and extended emissions, a nuclear spectrum would be confined to 
around 200 pc, while an extended spectrum would consider scales around and above 1 kpc. Hence, considering the 3" aperture size of SDSS, the galaxies whose spectra would refer solely to the nuclear region would be those at $z \lesssim 0.003$ (considering the same cosmological parameters as mentioned at Section 21. We test the aperture effects of our sample in Appendix B.1.

This emission, that can extend to kpc scales, is not associated with low-luminosity AGNs, that bona fide LINERs are, and thus has been associated with the photoionization by HOLMES, which are the dominant source of ionizing photons in quiescent regions, after star formation has ceased (Belfiore et al., 2016). These stars can produce enough photoionizing photons to reproduce a LINER-like spectral profile (Binette et al., 1994b; Stasińska et al., 2008) through the interstellar medium in a more dispersed way, though the number density of such stars is still unclear. The spatial distribution of HOLMES follows the overall stellar distribution, and their coincidence to the observed surface brightness profiles in the radial extension of the galaxies depends also on how the gas clouds are distributed relative to such old stars (Yan and Blanton, 2012). Some of the results that favour the hypothesis that HOLMES represent the diffuse source of ionizing photons, observed in extended emitting regions, are the tight correlation between the observed continuum and the line emission in these LINER-like objects and the $\mathrm{EW}(\mathrm{H} \alpha)$ in the range predicted by models that consider those components (Belfiore et al., 2016).

There are other candidates considered in the explanation of this excitation that is not originated from an AGN, such as X-ray binaries, extreme horizontal branch stars, and ultraluminous X-ray sources (Yan and Blanton, 2012). However, Sarzi et al. (2010) argue that the first two objects would produce much fewer photoionizing photons than HOLMES, while the last is too rare to be abundant as it would be required to explain the observations. Hence, such alternative options are unlikely the objects responsible for the detection of LINER-like emission far from the nucleus of a galaxy, since their contribution is overall negligible unless the density number of HOLMES has been widely over-predicted in the models (Belfiore et al., 2016).

Therefore, the objects that lie in the LINER loci of the standard diagnostic diagrams might be ionized by low ionization AGN or by hot evolved stars, or even by both mechanisms that are often found in early-type galaxies (see the integral field spectra of both mechanisms at Ricci et al., 2015). Considering only the emission-line ratios of these dia- 
grams, it is not possible to differentiate between these two power sources, and such problem persists in the alternative diagnostic diagrams proposed in the next Section, which are based in the same axes of the traditional diagrams. However, there are diagnostic diagrams in the literature that intend to solve such problem by considering parameters such as $\mathrm{EW}(\mathrm{H} \alpha)$ (WHAN diagram by Cid Fernandes et al., 2010) and $\mathrm{D}_{\mathrm{N}}(4000)$ (DEW diagrams by Stasińska et al., 2008), both mentioned at Section 1.2.2.

Although the LINERs might be less luminous in line emission (since the contribution of old stellar populations is often not considered) and might dominate in scales smaller than 100 pc (Yan and Blanton, 2012), the results from Agostino and Salim (2019) indicate that many of the low ionization AGNs are indeed not emitting strong lines, in a way that they are not classified by the standard diagnostic diagrams. However, this same work finds that the distribution of the X-ray detected AGNs among Seyferts and LINERs is similar, which confirms the existence of LINERs in large quantities, associated with a phase of lower activity of the central SMBH.

More than solely the contamination of stellar populations (young or old), another reason to not associate the intensity of the emission lines directly to the power of the AGN is the presence of diffuse ionized gas or shocks, that would also contribute to the emission-line intensity in case they are present in the region being integrated to produce the observed spectrum. These two sources of photoionization would also contaminate a star-forming region object, making their lines seem stronger and, consequently, misclassifying such objects as AGNs. Nevertheless, though they affect the classification based on the standard diagnostic diagrams, they are never the dominant power source of galactic nuclei.

The DIG contribution is prevalent in the case of star-forming objects, and would probably come from extra-planar emission, which is more critical in the case of edge-on systems, or from the inter-arm regions, relevant to both face- and edge-on dispositions (Belfiore et al., 2016; Zhang et al., 2017).

As for the shocks, they can be associated with stellar processes (as a consequence of stellar winds and supernovae), to AGN processes (through radiative winds, magnetic winds, jets, and outflow consequences in general), or even to external processes (as interacting galaxies, ram stripping, mergers and so on). One way to detect the presence of shocks is by comparing the velocity width differences among distinct emission lines, which should be systematically similar if the shocks are affecting their kinematics, with a probable increase 
in the gas velocity dispersion (Yan and Blanton, 2012). Thus, a correlation between the line ratio and the velocity dispersion can be a useful test to search for shock ionization (Monreal-Ibero et al., 2006, Rich et al., 2011, 2014), though in some cases of early-type galaxies and spheroidal symmetries of the system, the velocity dispersion might have a radial dependence as a consequence of gravitational virial motion. Another option would be to look for a localized increase in the velocity dispersion of the gas in comparison to the one of the stars (Belfiore et al., 2016).

The last very likely cause of misclassification of objects when using the traditional diagnostic diagrams is the quality of the data. This issue has been discussed when we mention the contamination of other sources of ionization or the difficulty in positioning objects with weak emission lines. However, the main problem is that the consequences of such difficulties are not equally distributed among the objects, with LINER-like objects being the ones mostly affected by the non detection of their weak lines, as already discussed. Therefore, as pointed out by Cid Fernandes et al. (2011), the cuts with regards to S/N (not so different from the cut in the uncertainty of the emission-line ratio that we consider to our sample) might introduce several selection effects that bias the sample distribution. Hence, we emphasize that the main concern of our sample is to study the position of objects that we can have more reliability with regard to the quality of the information obtained from their spectra, and we do not intend to propose any demographic studies because we are aware that they would be incomplete and biased.

\subsection{Alternative diagnostic diagrams}

In the present Section, we will introduce the alternative diagnostic diagrams proposed by this work. These diagrams are based on the same optical emission line ratios that have been widely used in the literature with the standard diagnostic diagrams - $[\mathrm{O} \mathrm{III}] / \mathrm{H} \beta$, $[\mathrm{N} \mathrm{II}] / \mathrm{H} \alpha,[\mathrm{S} \mathrm{II}] / \mathrm{H} \alpha$, and $[\mathrm{O} \mathrm{I}] / \mathrm{H} \alpha$ - as these ratios are known to best separate the objects, while minimizing the uncertainties due to reddening and flux calibrations (see Section 1.2.1). We will show that linear combinations of these line ratios can help to better understand the objects and their distinct classes.

In this Section, we will discuss the advantages and caveats of the alternative diagnostic diagrams and borderlines proposed. First, we will introduce diagnostic diagrams that 
require all the line ratios from the traditional diagnostic diagrams. Then, we present more economic diagrams that have been proposed in the literature.

The color code for the different classes of objects will be as in the previous Section, with star-forming nuclei in green, transition objects in black, Seyferts in blue, and LINERs in red. When Seyferts and LINERs are not distinguished, AGNs will be color coded in magenta. These color codes will be assigned based on the outcome of each of the classifications proposed.

We will consider the following additive and subtractive combination of oxygen line ratios:

$$
\begin{gathered}
\log \left(\frac{[\mathrm{O} \mathrm{III}]}{\mathrm{H} \beta}\right)-\log \left(\frac{[\mathrm{O} \mathrm{I}]}{\mathrm{H} \alpha}\right)=\log \left(\frac{[\mathrm{O} \mathrm{III}]}{[\mathrm{O} \mathrm{I}]}\right)+\log \left(\frac{\mathrm{H} \alpha}{\mathrm{H} \beta}\right), \\
\log \left(\frac{[\mathrm{O} \mathrm{III}]}{\mathrm{H} \beta}\right)+\log \left(\frac{[\mathrm{O} \mathrm{I}]}{\mathrm{H} \alpha}\right)
\end{gathered}
$$

and the following additive and subtractive combinations of the nitrogen and sulfur line ratios:

$$
\begin{gathered}
\log \left(\frac{[\mathrm{N} \mathrm{II}]}{\mathrm{H} \alpha}\right)+\log \left(\frac{[\mathrm{S} \mathrm{II}]}{\mathrm{H} \alpha}\right) \\
\log \left(\frac{[\mathrm{N} \mathrm{II}]}{\mathrm{H} \alpha}\right)-\log \left(\frac{[\mathrm{S} \mathrm{II}]}{\mathrm{H} \alpha}\right)=\log \left(\frac{[\mathrm{N} \mathrm{II}]}{[\mathrm{S} \mathrm{II}]}\right) .
\end{gathered}
$$

Equation 3.16, relates the oxygen emission lines with regard to Balmer recombination lines similar to what Heckman (1980) proposed when defining LINERs (see Section 1.1.2). This is a good indicator of the ionization level, since it compares the intensities of neutral and twice ionized oxygen. The Balmer decrement $(\mathrm{H} \alpha / \mathrm{H} \beta)$ for the objects in our sample is shown in Figure 3.12 . We notice that the values are in a larger range than the expectation of $\mathrm{H} \alpha / \mathrm{H} \beta \sim 2-4$. This is probably due to the fact that the fluxes provided by the MPAJHU catalog where corrected for foreground galactic reddening according to O'Donnell (1994). Many authors use the estimate that $\mathrm{H} \alpha / \mathrm{H} \beta \sim 2.85$ for pure photoionization (as expected from $\mathrm{H}$ II regions) and $\mathrm{H} \alpha / \mathrm{H} \beta \sim 3.1$ for AGNs (photoionization and collisions being considered) to correct the fluxes of the observed extragalactic objects (Osterbrock and Ferland, 2006). However, O'Donnell (1994) uses a different extinction law, and that is the reason for the values of the Balmer decrement in our sample to be in a relatively wide range.

The second and third axes presented, from Equations 3.17 and 3.18, are related to the excitation temperature of the gas. Since they are the sum of the log of the emission 


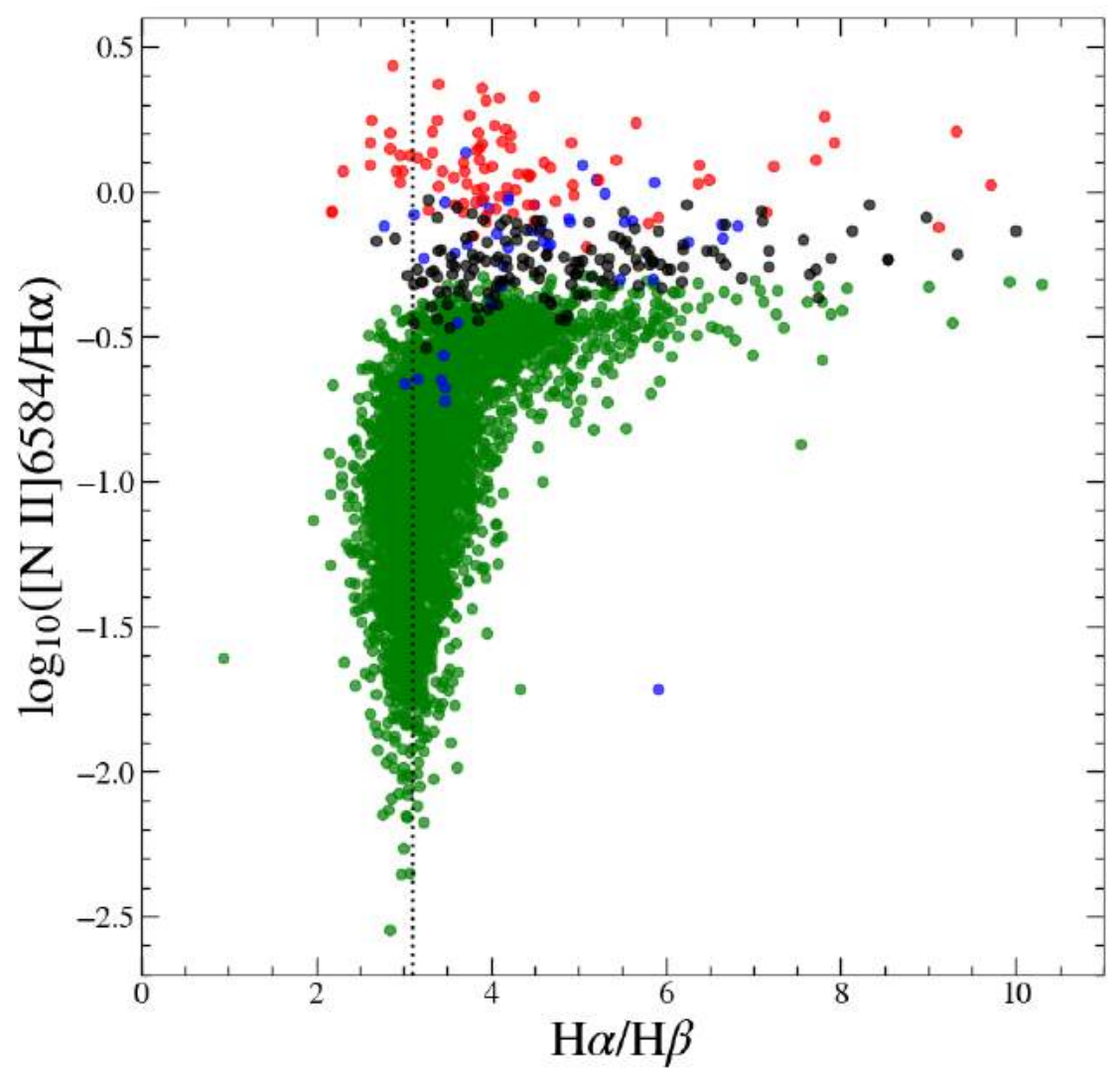

Figure 3.12: $[\mathrm{N} I \mathrm{II}] / \mathrm{H} \alpha$ line ratio versus the Balmer decrement $\mathrm{H} \alpha / \mathrm{H} \beta$ for our sample (see Section 2.3). Points in green are star-forming nuclei, in red are LINERs, in blue are Seyfert nuclei, and in black are transition objects, according to the lines proposed by Kewley et al. (2001) (see Equation 3.1), Kauffmann et al. (2003) (see Equation 3.4), and Cid Fernandes et al. (2010) (see Equation 3.14). Black dashed line shows $\mathrm{H} \alpha / \mathrm{H} \beta=3.1$.

line ratios, the greater the sum the harder the ionization field, resulting in higher nebular temperatures observed.

As for Equation 3.19, the ratio $[\mathrm{N}$ II $] /[\mathrm{S} \mathrm{II}]$ has been used as an abundance indicator, since nitrogen is mainly a secondary nuclear product and sulfur is a primary species, in a way that the ratio is an index that indicates the degree of secondary processing (Jensen et al. 1976). It is also sensitive to the ionization parameter, as a consequence of the mismatch between the ionization potentials of $[\mathrm{N} \mathrm{II}]$ and $[\mathrm{S} \mathrm{II}]$ (Dopita et al., 2013). It is worth recalling that the wavelength proximity of emission lines minimizes the effects of attenuation, in a way that it is somewhat easier to obtain accurate determinations of the line ratio $[\mathrm{N} \mathrm{II}] /[\mathrm{S} \mathrm{II}]$ than $[\mathrm{O} \mathrm{III}] /[\mathrm{O} \mathrm{I}]$.

We have tested the four combinations of Equations 3.16 and 3.17 on the ordinate axis and Equations 3.18 and 3.19 on the abscissas of our alternative diagrams. However, the combination of Equation 3.16 with Equation 3.19 does not show clearly the star- 
forming sequence evident in the standard diagrams and does not separate the objects in their respective classes. The other diagrams are more neat and we will evaluate empirical borderlines that best distinguish the classes.

The aim of combining the emission-line ratios from the three standard diagnostic diagrams is to synthesize all the information from the three individual diagrams while minimizing ambiguities. However, we emphasize that it is still useful to compare different types of diagrams to better understand the cases of ambiguous classifications or cases near the borderlines.

Using all the emission line ratios requires data in which all the lines can be measured, and this can bias the sample to only strong emitters, as discussed previously in Section 3.1.3. This means that we cannot provide a complete demographic study of all galactic nuclei, since objects with strong emission lines are prioritized and we have seen that many nuclei have weak emission lines, being present in the Universe but not well represented in our sample.

For the different classification schemes proposed in this Section, we first show the alternative diagnostic diagram with data color-coded according to Kewley et al. (2001), Kauffmann et al. (2003) (or Kewley et al. 2006) and Cid Fernandes et al. (2010), as in Figure 3.10, in order to evaluate if the proposed axes improve the distinction between the classes. Then, we propose new borderlines in these alternative diagnostic diagrams and check how the data are distributed when switching back to the standard diagrams.

To define new borderlines, we have considered all the other classification schemes presented in Section 3.1.1 and evaluated the number of objects that classified differently between the standard diagram borderlines and our proposed borderlines. Nevertheless, the classification scheme of Kewley et al. (2001), Kauffmann et al. (2003) (or Kewley et al. 2006) and Cid Fernandes et al. (2010) presents the fewest misclassification cases when comparing the BPT diagram with the VO-S and VO-O diagrams, and also classifies correctly all the objects in their desired classes. Hence, more weight was given to these borderlines to complement the study of the alternative classification schemes.

\subsection{1 $([\mathrm{O} \mathrm{III}] / \mathrm{H} \beta) \times([\mathrm{O} \mathrm{I}] / \mathrm{H} \alpha)$ vs. $([\mathrm{N} \mathrm{II}] / \mathrm{H} \alpha) \times([\mathrm{S} \mathrm{II}] / \mathrm{H} \alpha)$}

The first alternative diagnostic diagram has a similar distribution of objects as in the standard diagnostic diagrams, as can be seen in Figure 3.13 . Objects with harder 
ionization field will present stronger emission-line ratios, and so the AGNs are expected to be located on the upper right locus while star-forming galaxies will have lower values for the individual and summed line ratios, occupying the lower left locus. Therefore, we have chosen to maintain the shape of the traditional borderlines, by proposing a rectangular hyperbola to distinguish star-forming nuclei from AGNs, explicit in Equation 3.20, and a straight line with positive inclination to differentiate Seyferts from LINERs, shown in Equation 3.21. We chose to leave most of the transition objects in the star-forming locus in order to have fewer cases of misclassification on comparing with the standard diagnostic diagrams, thus, the curve that defines the boundary of star-forming regions does not follow the star-forming sequence so closely.

$$
\begin{gathered}
\log \left(\frac{[\mathrm{O} \mathrm{III}]}{\mathrm{H} \beta}\right)+\log \left(\frac{[\mathrm{O} \mathrm{I}]}{\mathrm{H} \alpha}\right)=\left(\frac{0.45}{[\log ([\mathrm{N} \mathrm{II}] / \mathrm{H} \alpha)+\log ([\mathrm{S} \mathrm{II}] / \mathrm{H} \alpha)]-0.2}\right)-0.1 \\
\log \left(\frac{[\mathrm{O} \mathrm{III}]}{\mathrm{H} \beta}\right)+\log \left(\frac{[\mathrm{O} \mathrm{I}]}{\mathrm{H} \alpha}\right)=1.5\left[\log \left(\frac{[\mathrm{N} \mathrm{II}]}{\mathrm{H} \alpha}\right)+\log \left(\frac{[\mathrm{S} \mathrm{II}]}{\mathrm{H} \alpha}\right)\right]-0.2 .
\end{gathered}
$$

Even though the star-forming sequence is present in Figure 3.13 , in this case the objects go from a lower part of the diagram to the upper right to then fall down again and maintaining approximately the same range of values for the abscissa. In the standard diagrams, the anti-correlation between metallicity and ionization gave a much stronger sequence from high $[\mathrm{O}$ III $] / \mathrm{H} \beta /$ low $[\mathrm{N} \mathrm{II}] / \mathrm{H} \alpha$ to low $[\mathrm{O}$ III $] / \mathrm{H} \beta /$ high $[\mathrm{N} \mathrm{II}] / \mathrm{H} \alpha$. The observed behaviour can be explained empirically by the analysis of the star-forming sequence in the VO-O diagram, which shows that there is no good correlation between $[\mathrm{O} \mathrm{III}] / \mathrm{H} \beta$ and $[\mathrm{O} \mathrm{I}] / \mathrm{H} \alpha$.

The boundaries were defined based on the comparison of how many objects were misclassified when we went back to inspect them in the traditional diagnostic diagrams. Though we have tested all the classification schemes presented in this work in our alternative diagrams, we have chosen to show only the comparison with the lines from Kewley et al. (2006) and Cid Fernandes et al. (2010).

Figure 3.14 shows the consequences of the classification scheme proposed in Figure 3.13 with Equations 3.20 and 3.21 for the standard diagnostic diagrams. Note that we are not considering a separate region of transition objects because this class overlaps significantly with star-forming galaxies and, to a lesser extent, with AGNs in Figure 3.13. Hence, the region of the BPT diagram (left panel) between the Kewley et al. (2001) and Kauffmann 


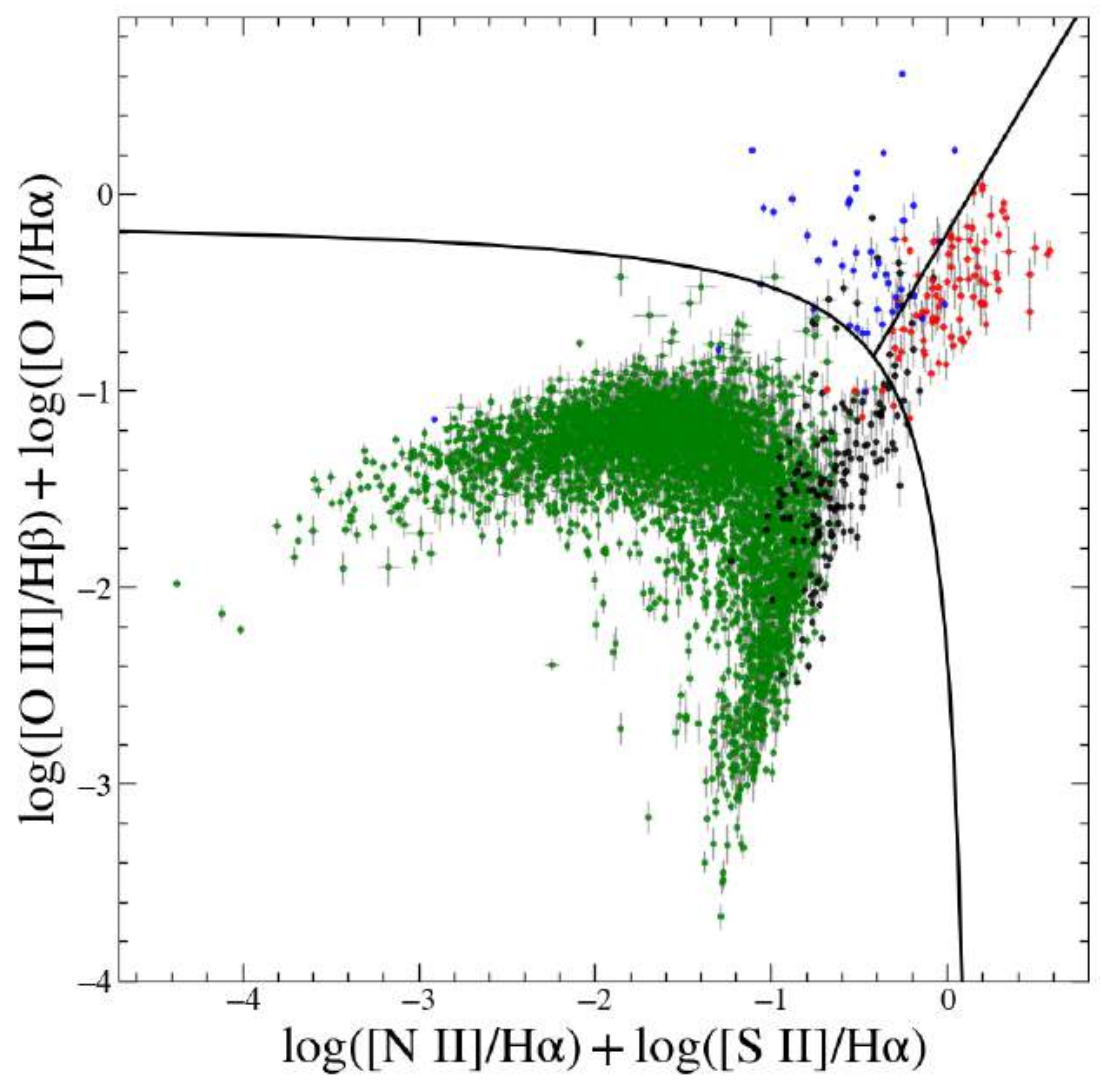

Figure 3.13: Alternative diagnostic diagram with the sum of the emission line ratios in the axes. Points in green are star-forming nuclei, in red are LINERs, in blue are Seyfert nuclei, and in black are transition objects, according to the lines proposed by Kewley et al. (2001) (see Equation 3.1), Kauffmann et al. (2003) (see Equation 3.4), and Cid Fernandes et al. (2010) (see Equation 3.14). Black lines are as in Equations 3.20 and 3.21 .

et al. (2003) lines now contains star-forming nuclei, Seyferts and LINERs, as expected, the majority being star-forming objects.

By comparing with the VO-S diagram of the middle panel of Figure 3.14, we notice that most of these objects in the transition region of the BPT diagram fall in the Seyfert and LINER region. In this case, because such objects are nearer the borderline and present high values of $[\mathrm{S} \mathrm{II}] / \mathrm{H} \alpha$, their misclassification seems to make more sense, in a way that though their $[\mathrm{N} \mathrm{II}] / \mathrm{H} \alpha$ ratio seems quite typical of star-forming objects, when their high ratio of sulfur is summed to the nitrogen ratio, it should result in a high value that has led them to be incorrectly classified as AGNs. Despite these two cases, the star-forming objects are reasonably classified, with a considerable part above the line from Kewley et al. (2001) as was already noticed in all of the cases where the classification scheme is based only on the BPT diagram and then applied to the VO-S diagram. It is also worth pointing out that some of the AGNs are mixed up, with several Seyfert nuclei in the LINERs cloud. 


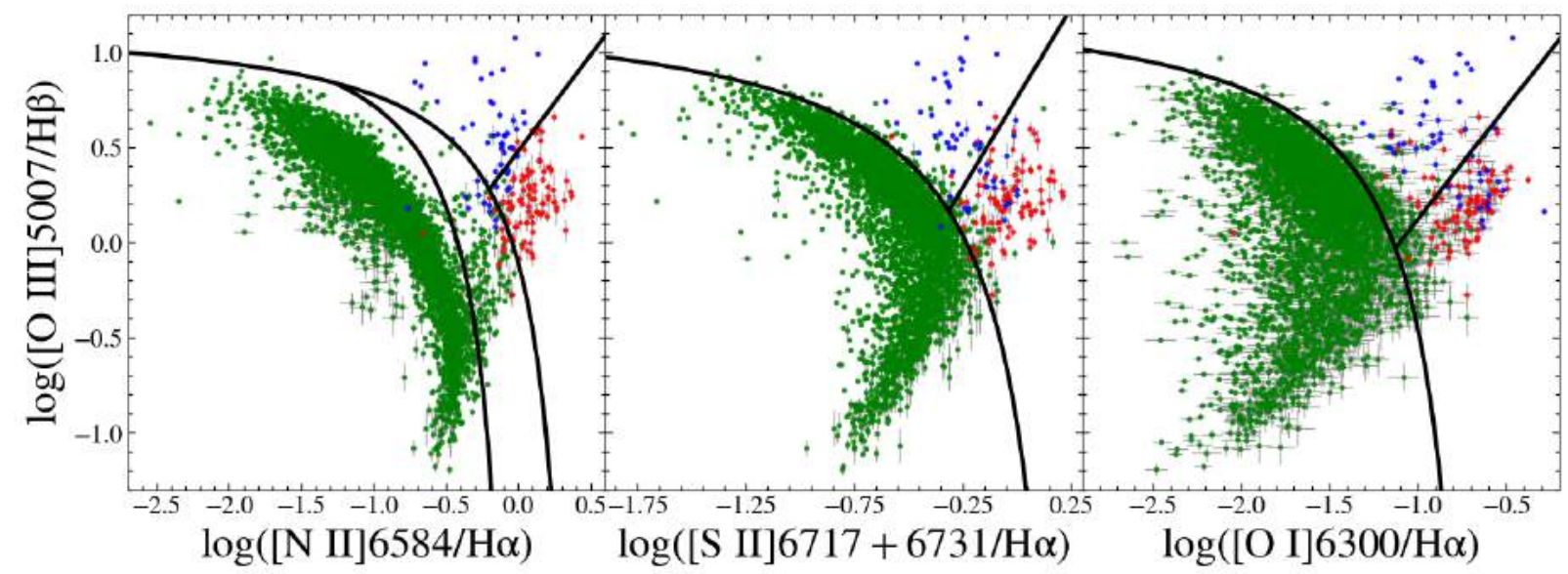

Figure 3.14: BPT (left), VO-S (middle), and VO-O (right) diagrams with the data color-coded according to Equations 3.20 and 3.21 (see Figure 3.13). Points in green are star-forming nuclei, in red are LINERs, and in blue are Seyferts.

These objects are the Seyferts that are occupying the transition region of the BPT diagram (left panel), and that therefore are represented as black points on Figure 3.13 .

In the right panel of Figure 3.14, we have the VO-O diagram for the data color-coded according to Figure 3.13, and the analysis follows our discussion for the middle panel. However, in this case the star-forming sequence is more extended outside the Kewley et al. (2001) boundary, and we do not see the misclassified Seyfert in the star-forming branch, only in the LINER region. Though there are some LINERs classified as Seyferts, we observe a larger number of Seyferts in the LINER locus.

This diagram is useful because it gather all the information from the three standard diagnostic diagrams into a single plot, with a classification scheme that is successful at classifying galactic nuclei considering all the traditional emission-line ratios avoiding the ambiguity issues of using all the lines in different classification schemes that might not agree. There is a neat gap between the star-forming sequence and the AGNs in Figure 3.13, and Seyferts and LINERs are also properly distinguished.

\section{$3.2 .2([\mathrm{O} \mathrm{III}] / \mathrm{H} \beta) \times([\mathrm{O} \mathrm{I}] / \mathrm{H} \alpha)$ vs. $([\mathrm{N} \mathrm{II}] / \mathrm{H} \alpha) /([\mathrm{S} \mathrm{II}] / \mathrm{H} \alpha)$}

The next alternative diagnostic diagram presented is useful for defining a region of transition objects and to distinguish star-forming nuclei from AGN. This graph has the sum of the oxygen line ratios on one axis as in Figure 3.13 , but the difference between $[\mathrm{N} \mathrm{II}] / \mathrm{H} \alpha$ and $[\mathrm{S} \mathrm{II}] / \mathrm{H} \alpha$ on the other. As shown in Figure 3.15, it is not possible to distinguish Seyferts and LINERs based on the $[\mathrm{N} \mathrm{II}] /[\mathrm{S}$ II $]$ ratios alone. 


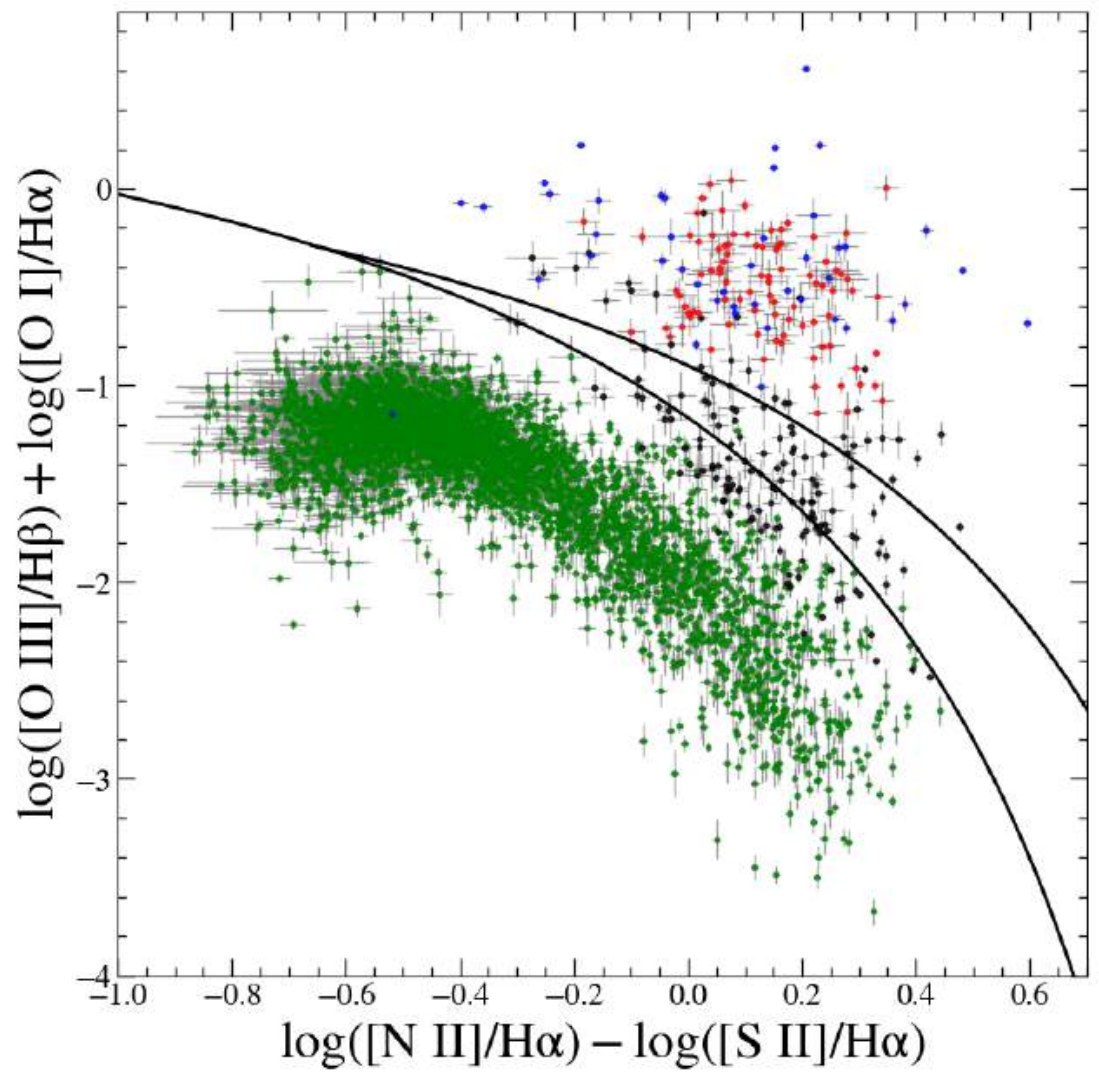

Figure 3.15: Alternative diagnostic diagram with the sum of the oxygen line ratios in the ordinate axis and the difference between nitrogen and sulfur line ratios in the abscissa. Points in green are star-forming nuclei, in red are LINERs, in blue are Seyfert nuclei, and in black are transition objects, according to the lines proposed by Kewley et al. (2001) (see Equation 3.1), Kauffmann et al. (2003) (see Equation 3.4), and Cid Fernandes et al. (2010) (see Equation 3.14). The black borderline that differentiates star-forming objects from transition objects is given by Equation 3.22 and the one that differentiates transition objects from AGNs is given by Equation 3.23 .

First, we define a region for transition objects, shown in Figure 3.15. Maintaining the shape of a rectangular hyperbola, the objects that are below the following expression will be classified as star-forming objects:

$$
\log \left(\frac{[\mathrm{O} \mathrm{III}]}{\mathrm{H} \beta}\right)+\log \left(\frac{[\mathrm{O} \mathrm{I}]}{\mathrm{H} \alpha}\right)=\left(\frac{3.5}{[\log ([\mathrm{N} \mathrm{II}] / \mathrm{H} \alpha)-\log ([\mathrm{S} \mathrm{II}] / \mathrm{H} \alpha)]-1.2}\right)+1.6
$$

Moreover, transition objects can be differentiated from AGN by using:

$$
\log \left(\frac{[\mathrm{O} \mathrm{III}]}{\mathrm{H} \beta}\right)+\log \left(\frac{[\mathrm{O} \mathrm{I}]}{\mathrm{H} \alpha}\right)=\left(\frac{3.0}{[\log ([\mathrm{N} \mathrm{II}] / \mathrm{H} \alpha)-\log ([\mathrm{S} \mathrm{II}] / \mathrm{H} \alpha)]-1.5}\right)+1.1 .
$$

This classification scheme was proposed in order to minimize the number of star-forming nuclei, Seyferts, and LINERs inside the transition region, though we recognize that if the proposed transition region was wider, it would gather more transition objects. We believe that this might indicate the dominant power source in the nuclei that were classified 


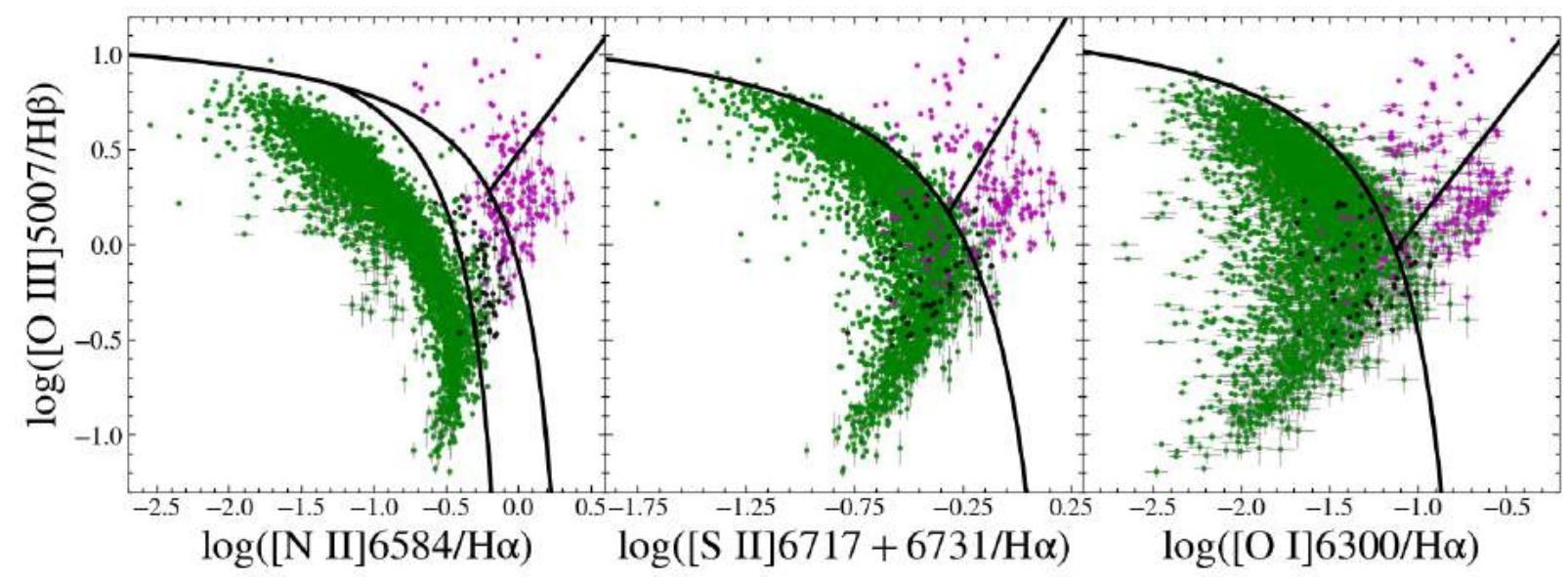

Figure 3.16: BPT (left), VO-S (middle), and VO-O (right) diagrams with the data color-coded according to Equations 3.22 and 3.23 Points in green are star-forming nuclei, in black are transition objects, and in magenta are AGNs.

as transition objects by the BPT diagram of Figure 3.10 but that do not reside in the transition region of our alternative diagnostic diagram.

Figure 3.15 thus distinguishes the classes according to their excitation temperature, with star-forming regions more to the bottom, associated with softer radiation fields, and the Seyferts and LINERs mixed above the transition objects, that lie in between H II regionlike objects and AGNs.

Figure 3.16 shows how the objects classified according to this new scheme are distributed in the standard diagnostic diagram. The BPT diagram from the left panel shows quite satisfactory results, with few misclassifications. We see some transition objects in both the star-forming and AGNs region, and we find cases of all three classes in the transition region, with $\mathrm{H}$ II region-like objects more to the left and AGNs more to the right, as expected. On the VO-S diagram of the middle panel, there are no misclassified objects in the AGN branch, but there are some transition objects in the star-forming branch. The VO-O diagram from the right panel of Figure 3.16 shows more scatter and misclassification, where the three different regions are populated by all the three classes that Equations 3.22 and 3.23 separate.

The diagram proposed in this Section is thus very important to make a clean selection of transition objects, helping to better understand the behavior of the different nuclei that occupy the transition region of the BPT diagram. Moreover, if the object one is analyzing resides the region of transition nuclei in Figure 3.15, it is crucial to use other diagnostic diagrams to determine in a more definite way which is the most relevant power source 
acting in it, as for example the diagram from Figure 3.13 that does not exhibit a separate transition region, but rather distributes such objects among star-forming nuclei or AGNs.

\section{$3.2 .3([\mathrm{O} \mathrm{III}] / \mathrm{H} \beta) /([\mathrm{O} \mathrm{I}] / \mathrm{H} \alpha)$ vs. $([\mathrm{N} \mathrm{II}] / \mathrm{H} \alpha) \times([\mathrm{S} \mathrm{II}] / \mathrm{H} \alpha)$}

The last alternative diagnostic diagram that combines all four emission-line ratios is based on the sum of the line ratios of nitrogen and sulfur (the same as Figure 3.13) and the difference between the oxygen emission line ratios.

In this first approach, we will consider the typical Balmer decrement that is found in extragalactic objects, instead of the range presented in Figure 3.12. The axis is then a comparison between twice ionized and neutral oxygen. This is a good indicator of the ionization parameter, and thus the main difference between Seyferts and LINERs, since [O III] tends to be strong in Seyferts (high-ionization AGNs) and weak in LINERs (lowionization AGNs), while the opposite occurs with the intensity of [O I], which is relatively strong in LINERs and weak in Seyferts. It was one of the criteria that Heckman (1980) used to define LINERs (see Section 1.1.2). The paper says that the [O I] line for LINERs "is at least one third as strong as" [O III]:

$$
3[\mathrm{O} \mathrm{I}] \geq[\mathrm{O} \mathrm{III}]
$$

Applying the logarithm scale on base 10 to the above sentence, we obtain

$$
\log [\mathrm{O} \text { III }]-\log [\mathrm{O} \text { I }] \leq 0.48
$$

Comparing to Equation 3.16, and considering that the Balmer decrement is about 3.1 for AGNs (Osterbrock and Ferland, 2006), we obtain that the theoretical limit between Seyferts and LINERs is approximately

$$
\log \left(\frac{[\mathrm{O} \mathrm{III}]}{\mathrm{H} \beta}\right)-\log \left(\frac{[\mathrm{O} \mathrm{I}]}{\mathrm{H} \alpha}\right) \approx 1.0 .
$$

Hence, Seyfert nuclei would be positioned above this division, and LINERs would be the objects below it.

As for the distinction between star-forming nuclei and AGNs, we are proposing a rectangular hyperbola as usual:

$$
\log \left(\frac{[\mathrm{O} \mathrm{III}]}{\mathrm{H} \beta}\right)-\log \left(\frac{[\mathrm{O} \mathrm{I}]}{\mathrm{H} \alpha}\right)=\left(\frac{5.55}{[\log ([\mathrm{N} \mathrm{II}] / \mathrm{H} \alpha)+\log ([\mathrm{S} \mathrm{II}] / \mathrm{H} \alpha)]-1.05}\right)+4.6
$$




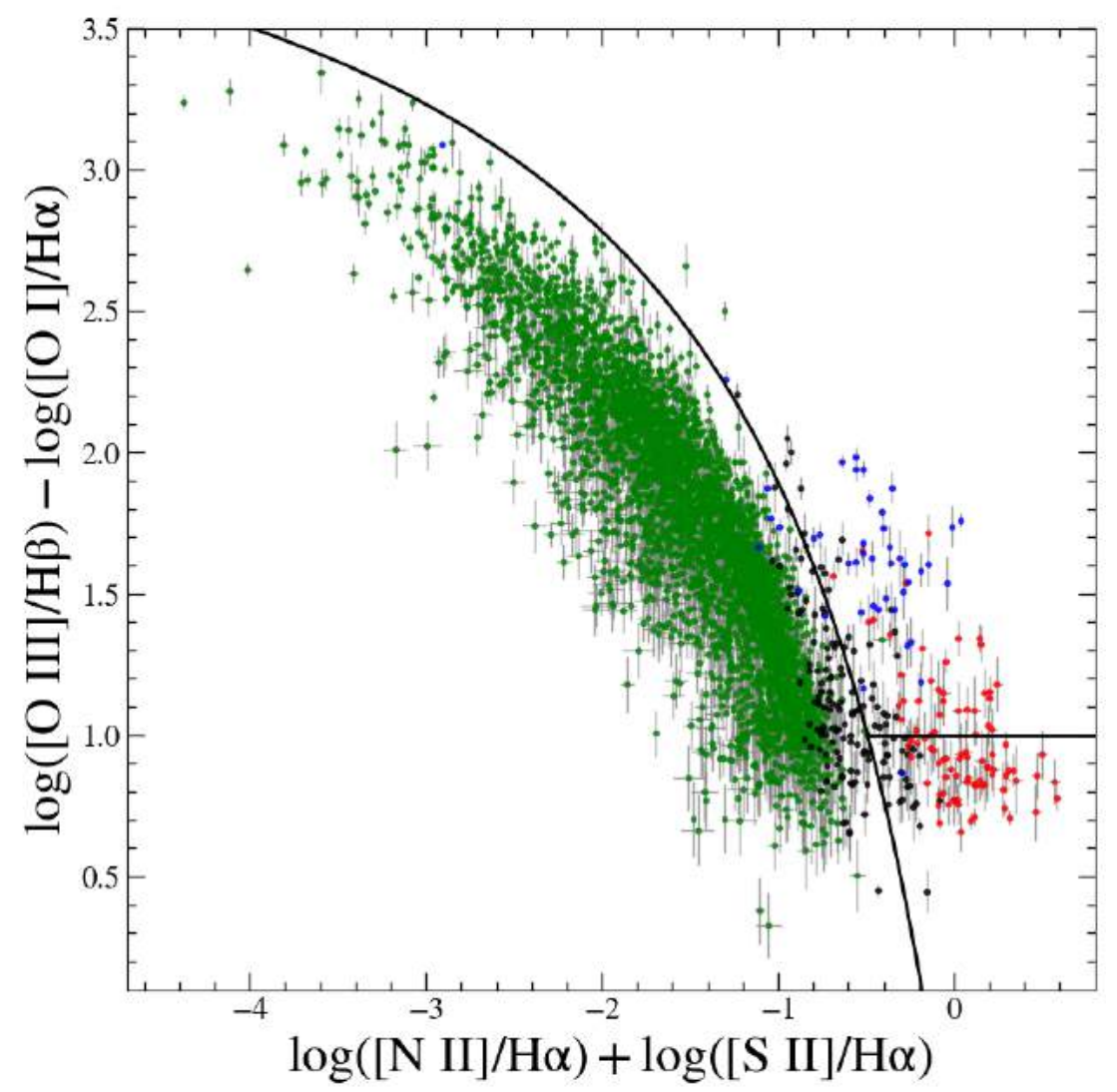

Figure 3.17: Alternative diagnostic diagram with the difference between the oxygen line ratios in the ordinate axis and the sum of nitrogen and sulfur line ratios in the abscissa. Points in green are star-forming nuclei, in red are LINERs, in blue are Seyfert nuclei, and in black are transition objects, according to the lines proposed by Kewley et al. (2001) (see Equation 3.1), Kauffmann et al. (2003) (see Equation 3.4), and Cid Fernandes et al. (2010) (see Equation 3.14). Black curve differentiates star-forming objects from AGNs as in Equation 3.25 proposed in this work, while the black straight line is a theoretical borderline between Seyferts and LINERs adapted from the proposal by Heckman $(1980)$, as in Equation 3.24

Figure 3.17 shows our alternative diagnostic diagram color coded according to Kewley et al. (2006) and Cid Fernandes et al. (2010), and we notice that the distinction between Seyferts and LINERs does not match that suggested by Equation 3.24, with several LINERs dominating the bottom part of the Seyfert locus. The diagram is mainly successful in separating the star-forming sequence from AGNs, though we see some Seyferts in the starforming locus, which are probably AGNs in lower metallicity environments. The diagram also separates the two classes of AGNs one from another, roughly parallel to the ordinate axis which traces ionization parameter.

We apply the classification scheme from Equations 3.24 and 3.25 to the traditional diagrams on Figure 3.18. The transition region in the BPT diagram of the left panel 


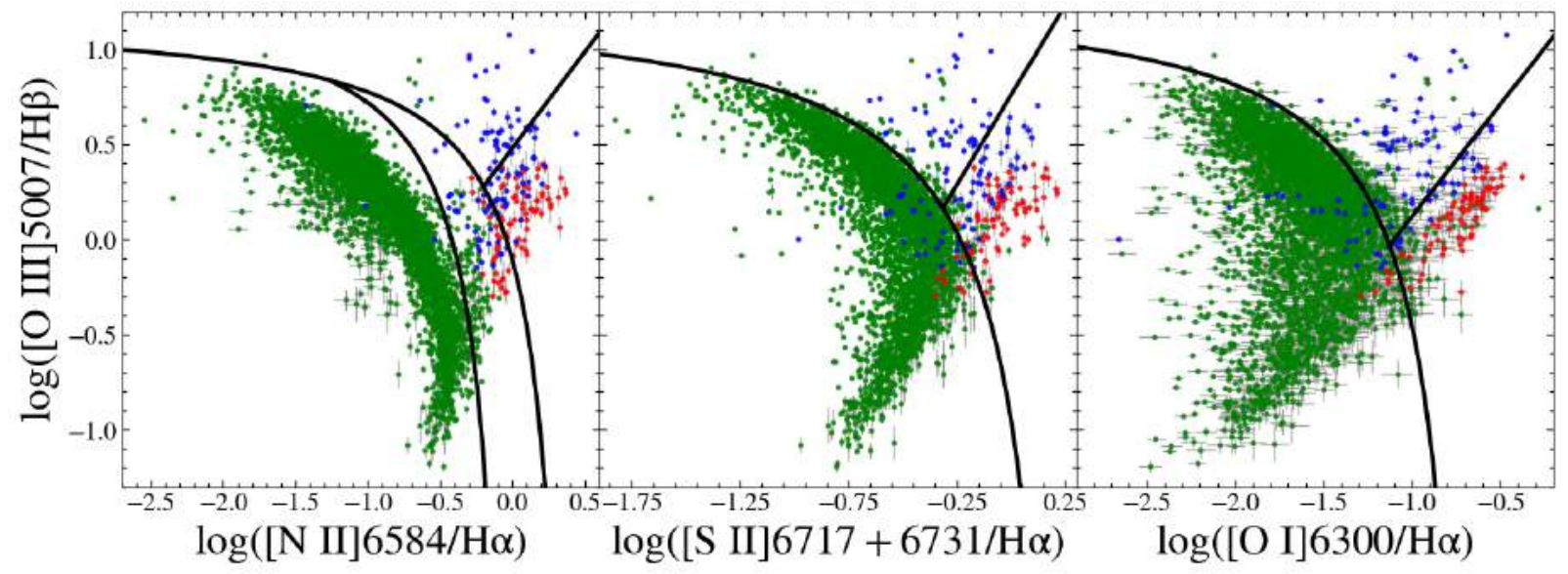

Figure 3.18: BPT (left), VO-S (middle), and VO-O (right) diagrams with the data color-coded according to Equations 3.24 and 3.25. Points in green are star-forming nuclei, in red are LINERs, and in blue are Seyferts.

is again populated by the three classes, with AGNs more to the right and H II regionlike objects closer to the star-forming sequence, as expected. There are three galaxies that were classified as Seyferts but lie in the star-forming sequence, and these objects are probably nearby the borderline of Equation 3.25. Figure 3.17 shows that the distinction between Seyferts and star-forming objects is fuzzy, with transition objects overlapping both classes, while for LINERs the transition objects fill a gap between star-forming objects and AGNs. Once again we notice some Seyferts with low $[\mathrm{N} \mathrm{II}] / \mathrm{H} \alpha$ being classified as star-forming objects, but other than that, the Seyfert loci seems to be in agreement with the classification scheme. The LINERs, however, have a significant population of Seyferts in the upper part of its locus, indicating that the borderline between these two types of AGN could be better constrained.

In the VO-S diagram from the middle panel of Figure 3.18, we observe more objects that were classified as AGNs in the star-forming branch, with the prevalence of Seyferts rather than LINERs that were mainly in the transition region of the left panel. Some objects classified as star-forming regions have high values of $[\mathrm{S} \mathrm{II}] / \mathrm{H} \alpha$, populating both Seyfert and LINER loci. Once again, there are no LINERs in the Seyfert locus, but there are several Seyferts in the upper part of the LINER locus.

The right panel of Figure 3.18 shows the VO-O diagram. Similarly to the middle panel, we have more Seyferts than LINERs in the star-forming sequence, and we have some objects classified as star-forming nuclei that lie far away from the star-forming branch, with high values of $[\mathrm{O}$ I $] / \mathrm{H} \alpha$ that can only be explained by an extended partially ionized zone typical 


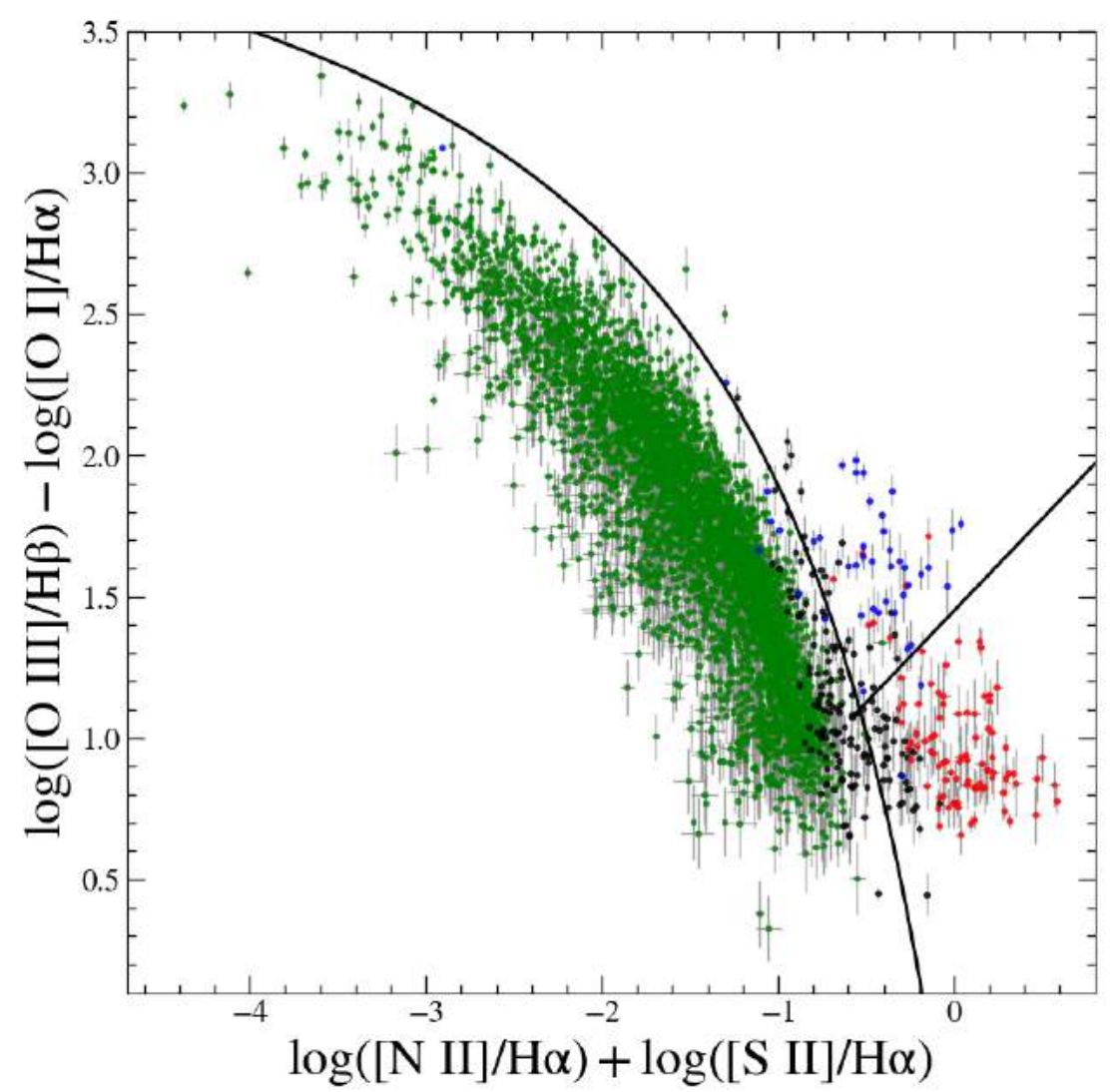

Figure 3.19: Alternative diagnostic diagram with the difference between the oxygen line ratios in the ordinate axis and the sum of nitrogen and sulfur line ratios in the abscissa. Points in green are star-forming nuclei, in red are LINERs, in blue are Seyfert nuclei, and in black are transition objects, according to the lines proposed by Kewley et al. (2001) (see Equation 3.1), Kauffmann et al. (2003) (see Equation 3.4), and Cid Fernandes et al. (2010) (see Equation 3.14). Black curve differentiates star-forming objects from AGNs as in Equation 3.25 and the black straight line differentiates Seyferts and LINERs as in Equation 3.26

for AGNs. This misclassification is probably due to the fact that the region near the borderline defined by Equation 3.25 is populated by scatter from all the different classes.

Moreover, objects with medium to high $[\mathrm{O}$ III $] / \mathrm{H} \beta$, low $[\mathrm{N} \mathrm{II}] / \mathrm{H} \alpha$ and high $[\mathrm{S} \mathrm{II}] / \mathrm{H} \alpha$ and $[\mathrm{O} \mathrm{I}] / \mathrm{H} \alpha$ will populate an intermediate region of the alternative diagnostic diagram of Figure 3.17, in a way that their differences are more evident when we consider each line ratio separately in the standard diagnostic diagrams. Thus, combining the line ratios is useful to avoid misclassification of objects that have a more typical behavior expected from star-forming nuclei and AGNs, but objects with more complex spectra might be misclassified. Hence, diagnostic diagrams in general are very useful for classifying large number of galaxies, but for specific cases it is more conclusive to analyze the whole spectrum besides other indicators. 


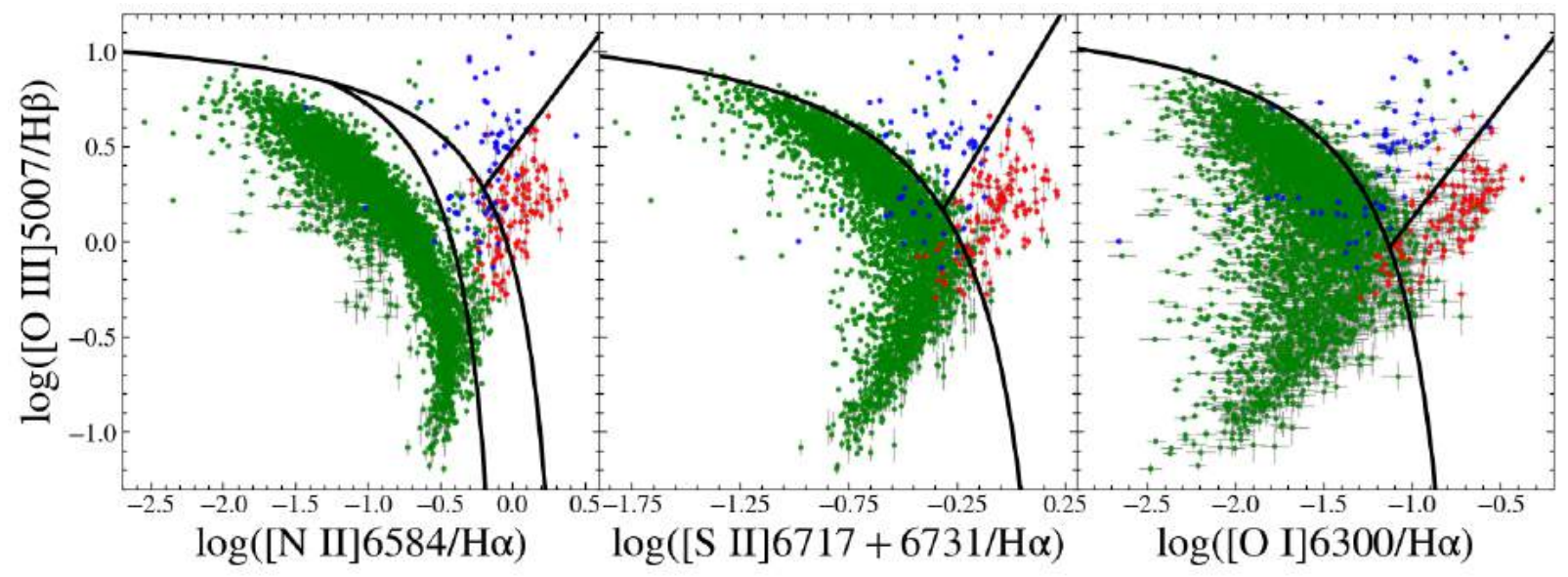

Figure 3.20: BPT (left), VO-S (middle), and VO-O (right) diagrams with the data color-coded according to Equations 3.25 and 3.26 . Points in green are star-forming nuclei, in red are LINERs, and in blue are Seyferts.

Equation 3.24 is based mainly on theoretical arguments. However, from Figure 3.12 we see that our sample does not show only objects with $\mathrm{H} \alpha / \mathrm{H} \beta \sim 3.1$, and from Figure 3.17 we see that the theoretical criterion does not completely distinguish Seyferts and LINERs for our sample. Moreover, there is a clean separation between these classes in our alternative diagram from Figure 3.17, and this separation is at a somewhat different location than suggested by Heckman (1980). We propose a straight line as follows:

$$
\log \left(\frac{[\mathrm{O} \mathrm{III}]}{\mathrm{H} \beta}\right)-\log \left(\frac{[\mathrm{O} \mathrm{I}]}{\mathrm{H} \alpha}\right)=0.65\left[\log \left(\frac{[\mathrm{N} \mathrm{II}]}{\mathrm{H} \alpha}\right)+\log \left(\frac{[\mathrm{S} \mathrm{II}]}{\mathrm{H} \alpha}\right)\right]+1.45
$$

This new division is shown in Figure 3.19 .

We applied the classification scheme of Figure 3.19 to the standard diagrams in Figure 3.20. Comparing the distinction between Seyferts and LINERs from the left panel, we see that we have less Seyferts in both the transition region and in the LINER locus. There is one LINER in the Seyferts locus, but from the large decrease of the Seyferts that were occupying the LINER locus in comparison to Figure 3.18 we can conclude that this empirical distinction is more reliable for AGN classification.

The VO-S diagram is shown in the middle panel of Figure 3.20. Some of the objects classified as Seyferts that lie in the star-forming sequence are now classified as LINERs, and though it is still a misclassification, at least it is in better agreement with the fact that both are AGNs. Again we have one LINER in the Seyfert locus, and several objects classified as Seyferts in the middle panel of Figure 3.18 while residing in the LINER region are now correctly classified as LINERs. 
The VO-O diagram is presented in the right panel of Figure 3.20, and the analysis is similar to what was described for the left and middle panels. In this case we have more LINERs in the Seyfert locus, which indicates a disagreement between the lines proposed by Kewley et al. (2006) for VO-S and VO-O. This is very interesting, since the $[\mathrm{O} \mathrm{I}] / \mathrm{H} \alpha$ ratio is the parameter most relevant in differentiating Seyferts and LINERs, as emphasized by Heckman (1980) that proposed the LINER classification. Therefore, when we consider the difference between the twice ionized and neutral oxygen, we are probably losing some important information about the intensity of the neutral oxygen with respect to other line ratios. Though the $[\mathrm{O}$ III $] /[\mathrm{O} \mathrm{I}]$ ratio is a good ionization parameter estimator, looking only at the intensity of $[\mathrm{O} \mathrm{I}] / \mathrm{H} \alpha$ has the benefit of assessing the presence and size of a partially ionized zone and consequently about the hardness of the radiation field of the central source. Also, since this diagram is the best discriminator among the standard diagnostic diagrams, the amount of star-forming objects being misclassified is rather troublesome.

It would be necessary to test the behaviour of other samples to check if this result is a consequence of some selection effects from our sample or if indeed the borderline from Equation 3.26 is more adequate than the theoretical boundary defined by Equation 3.24. One possible selection effect from our sample could be associated to the uncertainty requirement with regard to the neutral oxygen line, that might bias the sample to Seyferts with relatively strong $[\mathrm{O} \mathrm{I}]$.

\subsection{4 ([S II $] / \mathrm{H} \alpha)$ vs. $([\mathrm{N} \mathrm{II}] / \mathrm{H} \alpha)$}

The next alternative diagram we present has already been used in the literature by, e.g., Storchi-Bergmann and Pastoriza (1990), Dopita and Sutherland (1995), Groves et al. (2004), Dors et al. (2008), Pérez-Montero and Contini (2009), Dors et al. (2011), Dopita et al. (2013), and Richardson et al. (2014) with the main purpose of studying the abundance of sulfur and nitrogen or to test theoretical models. The axes of this diagram are the ratios of $[\mathrm{S} \mathrm{II}] / \mathrm{H} \alpha$ and $[\mathrm{N} \mathrm{II}] / \mathrm{H} \alpha$, and since both ratios depend on the metallicity and (to a lesser extent) on the ionization parameter (Storchi-Bergmann et al., 1994; Kewley and Dopita, 2002), it has been used as a tool to estimate the metallicity (Viironen et al. 2007).

This diagram is much more economical than the other alternative diagrams. Cid Fernandes et al. (2010) points out that many emission-line galaxies are often excluded from classification because of their weak [O III] and/or $\mathrm{H} \beta$ lines, leading to incompleteness in the 


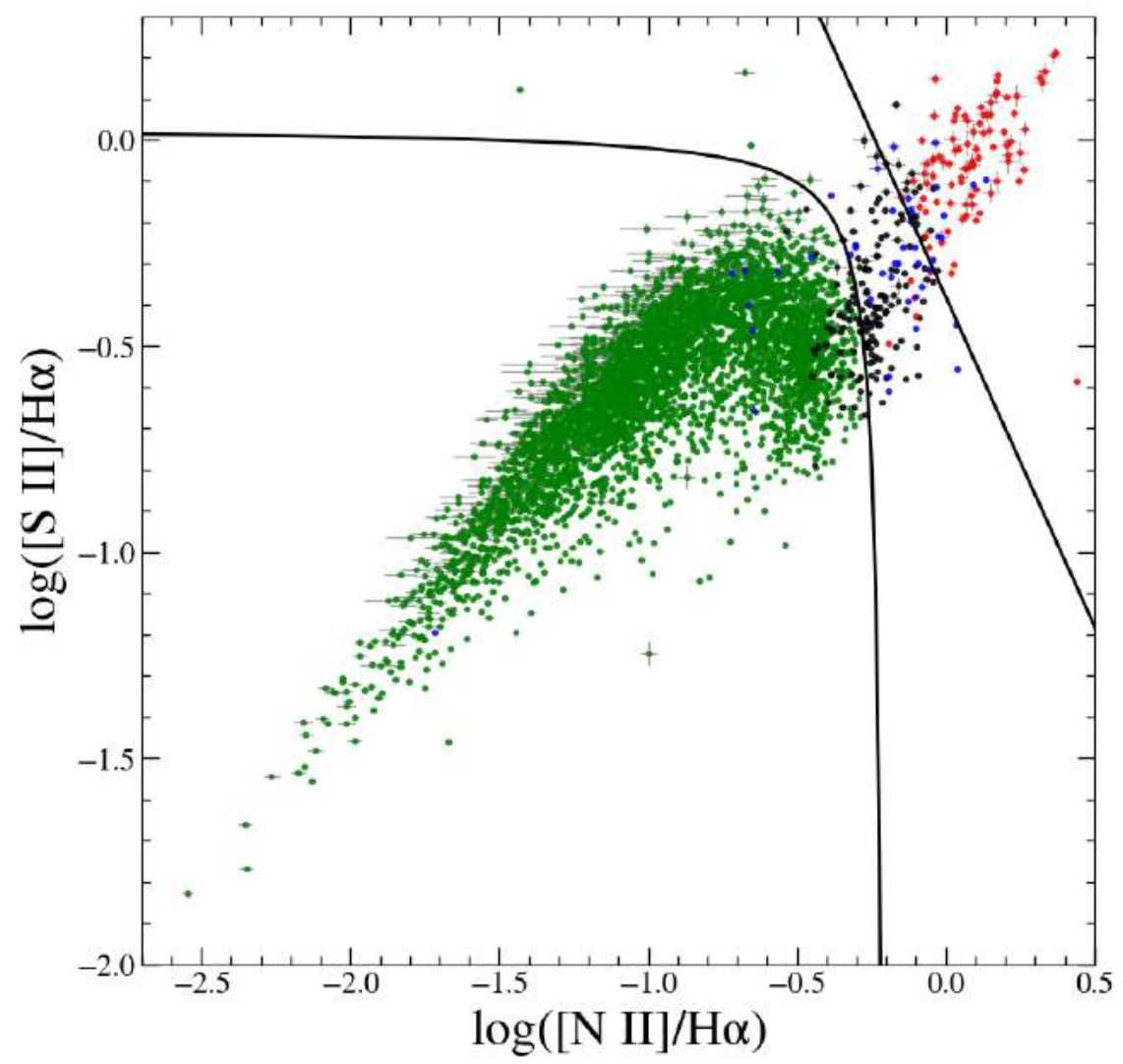

Figure 3.21: Alternative diagnostic diagram with the nitrogen and sulfur line ratios as axes. Points in green are star-forming nuclei, in red are LINERs, in blue are Seyfert nuclei, and in black are transition objects, according to the lines proposed by Kewley et al. (2001) (see Equation 3.1), Kauffmann et al. (2003) (see Equation 3.4), and Cid Fernandes et al. (2010) (see Equation 3.14). Black curve differentiates star-forming objects from Seyferts as in Equation 3.27 and the black straight line differentiates Seyferts from LINERs as in Equation 3.28

demographics of galactic nuclei. Hence, as in the case of the WHAN diagram, the $[\mathrm{N} \mathrm{II}] / \mathrm{H} \alpha$ versus $[\mathrm{S} \mathrm{II}] / \mathrm{H} \alpha$ diagram is more inclusive than those that require also the weaker emission lines. In cases where [O III] or $\mathrm{H} \beta$ are weak, absent or unavailable, this diagram is, thus, a good option for classifying a large fraction of galactic nuclei according to their dominant power source.

This diagram furthermore uses lines that are all very close in wavelength. Therefore, not only the effects of reddening and flux corrections will be negligible, but also this diagram can be used in case of limited spectral coverage.

The diagram is presented in Figure 3.21. A behavior that was not observed when considering the oxygen lines is that, in the case of Figure 3.21, the transition objects and Seyferts form a bridge between the star-forming sequence and the LINERs. Since the metallicity is the main parameter that determines the position of the data, in this diagram, 
this trend is probably related to the mass-metallicity relation (e.g. Tremonti et al., 2004; Matsuoka et al., 2018; Thomas et al., 2019). Objects toward the bottom left will be those with lower masses, and those toward the top right will be the more massive objects, associated with red-sequence elliptical galaxies that host LINERs (Schawinski et al., 2007; Ho, 2008; Heckman and Best, 2014). This explains the sequence from star-forming galaxies, transition objects, Seyferts and LINERs along the diagonal, as LINERs have more massive and more quiescent hosts compared to Seyferts.

Another important feature is the hook-like shape of the star-forming sequence as a consequence of the varying metallicity. This behavior was not observed by the previous works that considered this diagram because of differences in the sample selection, either due to a lower number of objects or objects that did not have enough spectral resolution for this trend to be evident.

The rectangular hyperbola drawn to separate H II region-like objects from Seyferts does not follow strictly the star-forming sequence, but follows the practice of rectangular hyperbolas proposed by Kewley et al. (2001) for the VO-S diagram and by Kauffmann et al. (2003) for the BPT diagram. We define star-forming regions as the objects that lie below:

$$
\log \left(\frac{[\mathrm{S} \mathrm{II}]}{\mathrm{H} \alpha}\right)=\left(\frac{0.07}{\log ([\mathrm{N} \mathrm{II}] / \mathrm{H} \alpha)+0.17}\right)+0.18,
$$

and we divide Seyferts and LINERs with a straight line defined by:

$$
\log \left(\frac{[\mathrm{S} \mathrm{II}]}{\mathrm{H} \alpha}\right)=-1.6 \log \left(\frac{[\mathrm{N} \mathrm{II}]}{\mathrm{H} \alpha}\right)-0.38 .
$$

The advantage of being economic comes at a cost of quality, which is obvious from the comparison with the standard diagrams in Figure 3.22. In general, we observe much ambiguously classified objects compared to when the oxygen lines were considered.

Figure 3.22 shows the classification scheme from Figure 3.21 as applied to the standard diagrams. In the BPT on the left panel, a few objects classified as Seyferts and one as LINER reside in the star-forming sequence. These Seyferts have relatively low $[\mathrm{N}$ II $] / \mathrm{H} \alpha$ and high $[\mathrm{S} \mathrm{II}] / \mathrm{H} \alpha$, and we chose to classify them as Seyferts because they were found in the top-right of the VO-S diagram shown in the middle panel of Figure 3.22. However, we emphasize that these particular objects should be analyzed individually to reach better conclusions about their nature. The transition region contains more Seyferts, though star-forming objects and LINERs are also present. There are only three Seyferts in the 


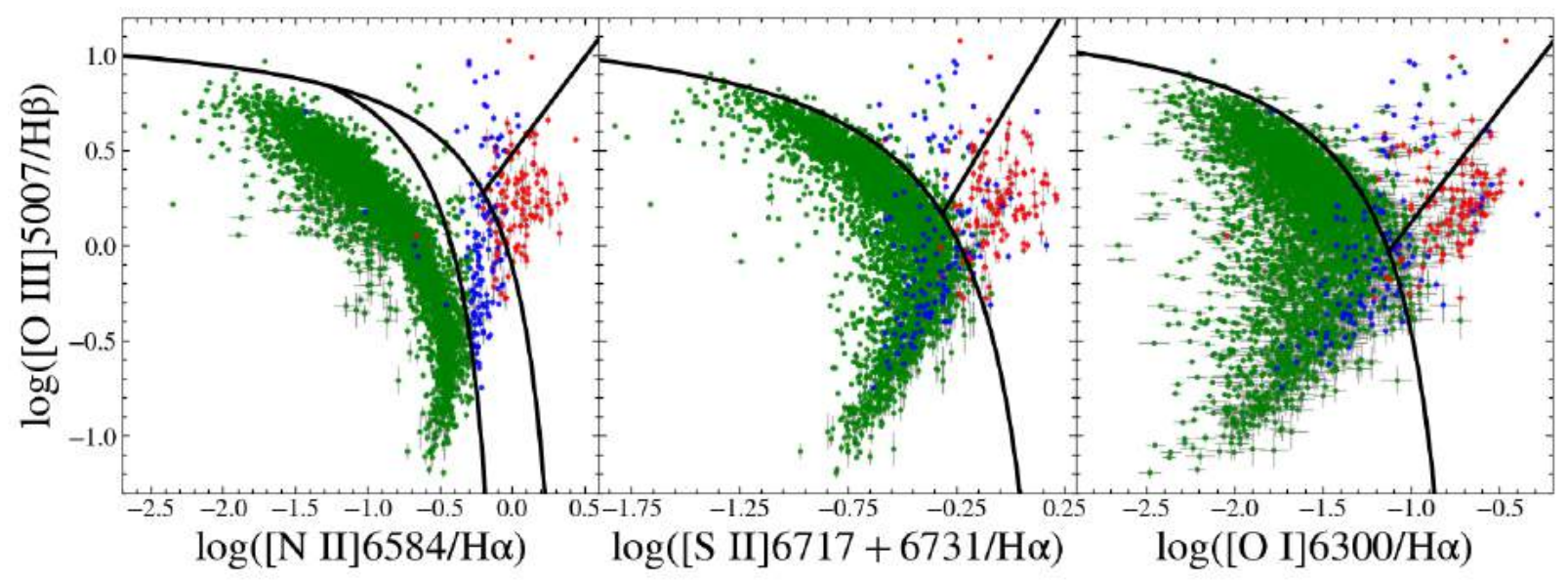

Figure 3.22: BPT (left), VO-S (middle), and VO-O (right) diagrams with the data color-coded according to Equations 3.28 and 3.27 . Points in green are star-forming nuclei, in blue are Seyferts, and in red are LINERs.

LINER locus but all other objects there are correctly classified. Some objects in the Seyfert locus are classified as star-forming regions with high values of $[\mathrm{N} \mathrm{II}] / \mathrm{H} \alpha$, though they also have high $[\mathrm{O} \mathrm{III}] / \mathrm{H} \beta$ ratios that are inconsistent with $\mathrm{H}$ II region-like objects. This issue is similar to what happened in the classification scheme of Stasińska et al. (2006) that was based only on the $[\mathrm{NII}] / \mathrm{H} \alpha$ ratio. We also find several LINERs in the Seyfert locus. Hence, though it is very interesting and unexpected that the transition objects overlap with the Seyfert nuclei in Figure 3.21, as a consequence Seyferts are difficult to classify in this diagram. In fact, no other BPT diagram that we analyzed showed this many Seyfert nuclei in the transition region.

The VO-S diagram is presented in the middle panel of Figure 3.22, and the starforming branch contains many more AGNs than in the left panel. We see several Seyferts that would probably be classified as transition objects by Kewley et al. (2006), and a few LINERs nearby the LINER locus. We also see many star-forming regions with high $[\mathrm{O}$ III $] / \mathrm{H} \beta$ in the Seyfert locus, with misclassified star-forming objects in the LINER locus closer to the star-forming sequence. There is also some mixture between Seyferts and LINERs along their loci.

From the VO-O diagram from the right panel of Figure 3.22, we see that this overlap is probably a consequence of distribution of the objects in the transition region of the BPT diagram. Although $[\mathrm{O} \mathrm{I}] / \mathrm{H} \alpha$ is usually the hardest ratio to measure, the VO-O diagram provides the most reliable diagnostic among the standard configurations (see Section 1.2.1). Not considering either of the oxygen line ratios in the classification scheme erases the 


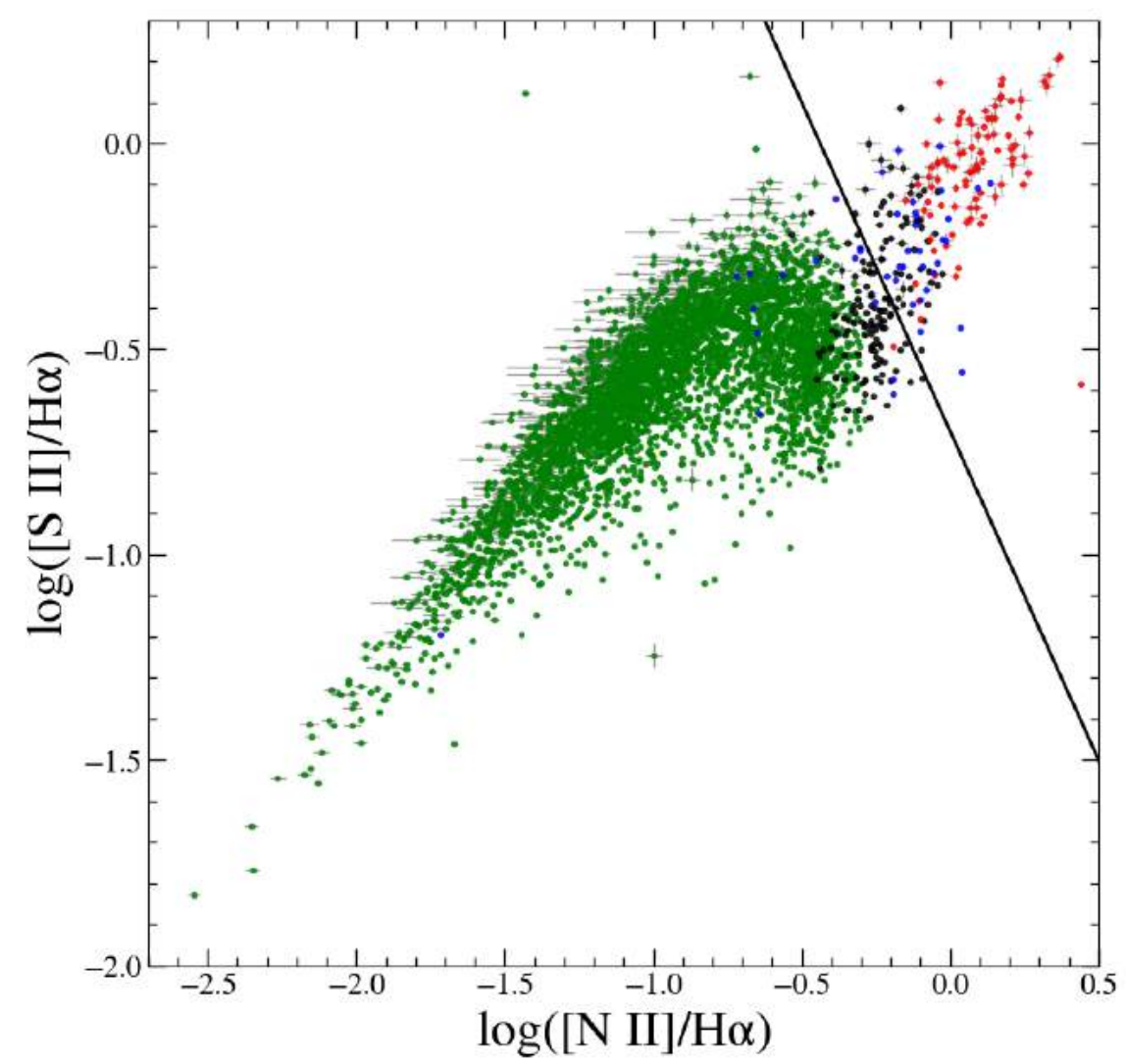

Figure 3.23: Alternative diagnostic diagram with the nitrogen and sulfur line ratios as axes. Points in green are star-forming nuclei, in red are LINERs, in blue are Seyfert nuclei, and in black are transition objects, according to the lines proposed by Kewley et al. (2001) (see Equation 3.1), Kauffmann et al. (2003) (see Equation 3.4), and Cid Fernandes et al. (2010) (see Equation 3.14). Black line separates star-forming nuclei from AGNs according to Equation 3.29 .

very sharp distinction between the loci, especially in comparison with the less economic alternative diagrams that consider all the emission lines. The LINERs and Seyferts are mixed and there are several objects color-coded as star-forming regions with too high $[\mathrm{O}$ I] $/ \mathrm{H} \alpha$ that are probably misclassified AGNs.

We can also propose a simpler separation between star-forming objects and AGNs, at the expense of separating Seyferts and LINERs by shifting the linear coefficient of Equation 3.28

$$
\log \left(\frac{[\mathrm{S} \mathrm{II}]}{\mathrm{H} \alpha}\right)=-1.6 \log \left(\frac{[\mathrm{N} \mathrm{II}]}{\mathrm{H} \alpha}\right)-0.7
$$

Figure 3.23 shows such classification scheme. According to this plot, all objects classified as star-forming nuclei with the criteria established by Kewley et al. (2006) and Cid Fernandes et al. (2010) are below Equation 3.29, but we find some Seyferts and one LINER in the star-forming sequence. No star-forming object is found above Equation 3.29, and 


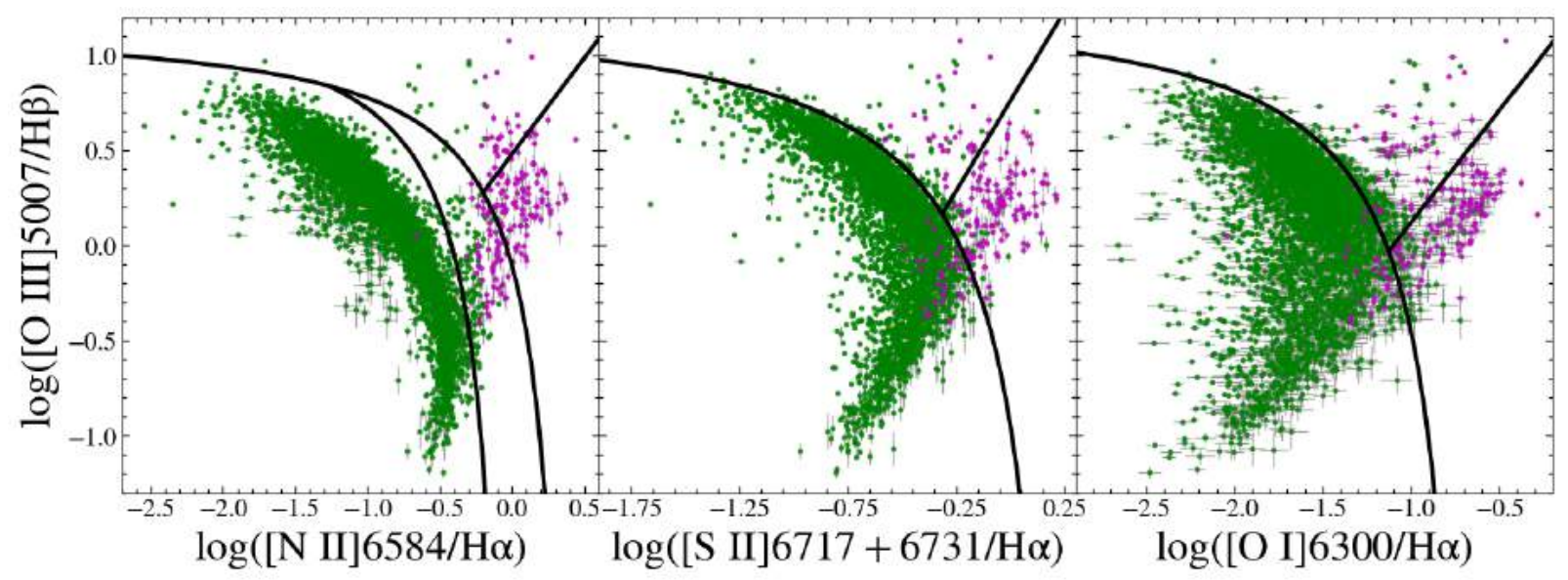

Figure 3.24: BPT (left), VO-S (middle), and VO-O (right) diagrams with the data color-coded according to Equation 3.29. Points in green are star-forming nuclei and in magenta are AGNs.

the transition objects are split among the star-forming and AGN loci.

It is worth calling attention to the particular objects classified by Kauffmann et al. (2003) as star-forming nuclei with low $[\mathrm{N} \mathrm{II}] / \mathrm{H} \alpha$ and high $[\mathrm{S} \mathrm{II}] / \mathrm{H} \alpha$. These are kept within the class of $\mathrm{H}$ II region-like objects in this simpler approach. Hence, in the BPT diagram in the left panel of Figure 3.24, they are correctly placed on the star-forming sequence, while in the VO-S and VO-O diagrams of the middle and right panels of Figure 3.24, respectively, they reside on the LINER region (in a way that they would be Seyferts according to the classification scheme of Equations 3.27 and 3.28.

Figure 3.24 shows the standard diagnostic diagrams according to the classification scheme of Equation 3.29, Therefore, in the BPT diagram of the left panel, the four starforming objects that were misclassified as Seyferts using the scheme proposed in Equations 3.27 and 3.28 (Figure 3.22) are now correctly classified as H II region-like objects, though one star-forming object that was misclassified as LINER remains an AGN in the starforming sequence. We have more star-forming objects in the transition region, where there were several Seyferts, and we have only AGNs in the LINER locus (as before, but with both Seyferts and LINERs). However, we have more Seyferts with low [N II]/H $\alpha$ erroneously classified as star-forming nuclei with high $[\mathrm{O}$ III $] / \mathrm{H} \beta$ line ratios.

As for the VO-S diagram from the middle panel of Figure 3.24, many nuclei that were classified in the middle panel of Figure 3.22 as Seyferts are now classified as star-forming objects. Such objects could be classified as transition objects, and we see much fewer AGNs in the star-forming sequence, though the misclassified LINERs with low $[\mathrm{S} \mathrm{II}] / \mathrm{H} \alpha$ 
and $[\mathrm{O} \mathrm{III}] / \mathrm{H} \beta$ still appear in the star formation branch. Many of these are bona fide Seyferts, and we see more misclassification cases in the Seyfert locus than in Figure 3.22 .

The right panel of Figure 3.24 shows the VO-O diagram for the classification scheme of Equation 3.29, As in the middle panel, there are much fewer misclassified Seyferts/AGNs in the star-forming branch when comparing to the right panel of Figure 3.22 , but more star-forming objects in the Seyfert locus. Thus, though the classification of star-forming objects is better with this approach and the classification of LINERs remains good, the classification of Seyferts, that lie in between in the alternative diagram (see Figures 3.21 or 3.23, is still troublesome.

We conclude that this economic diagram is useful, but as mentioned for the classification scheme of Stasińska et al. (2006) that considers only the $[\mathrm{N} \mathrm{II}] / \mathrm{H} \alpha$ line ratio, values from $\sim-0.5$ to $\sim-0.1$ lead to ambiguous classification, since both star-forming nuclei and AGNs have ratios in this range. This is one of the reasons for having an emission line sensitive to the ionization parameter, and $[\mathrm{OIII}] / \mathrm{H} \beta$ seems to give more precise results than $[\mathrm{S} \mathrm{II}] / \mathrm{H} \alpha$. Nevertheless, the proposed classification scheme is still useful as long as objects are not so close to this range of $[\mathrm{N} \mathrm{II}] / \mathrm{H} \alpha$ from about $\sim-0.5$ to $\sim-0.1$.

\section{$3.2 .5([\mathrm{O} \mathrm{III}] / \mathrm{H} \beta)$ vs. $([\mathrm{N} \mathrm{II}] /[\mathrm{S} \mathrm{II}])$}

The next alternative diagnostic diagram was proposed by Dopita et al. (2013). This diagram of Figure 3.25 compares the ratios of $[\mathrm{O} \mathrm{III}] / \mathrm{H} \beta$ and $[\mathrm{N} \mathrm{II}] /[\mathrm{S} \mathrm{II}]$. Hence, it combines information on the metallicity from the economic diagram of Figures 3.21 and 3.23 with the ionization parameter from the standard diagrams.

We will not propose any classification schemes for this diagram, because the different classes are not well separated as is immediately obvious from Figure 3.25. However, we present it here because it will be used to test the validity of our models in Section 3.3, as it is useful to discriminate between the influences of metallicity and ionization parameter separately. This is because models of H II region-like objects reach an upper envelope and then fold back on themselves in the standard diagnostic diagrams, but not in this alternative diagram. Hence, this diagram is useful for evaluating the values of certain parameters in the theoretical grids, even though it is not the best diagram for constraining the dominant power source.

We have shown that these alternative diagnostic diagrams cannot address issues that 


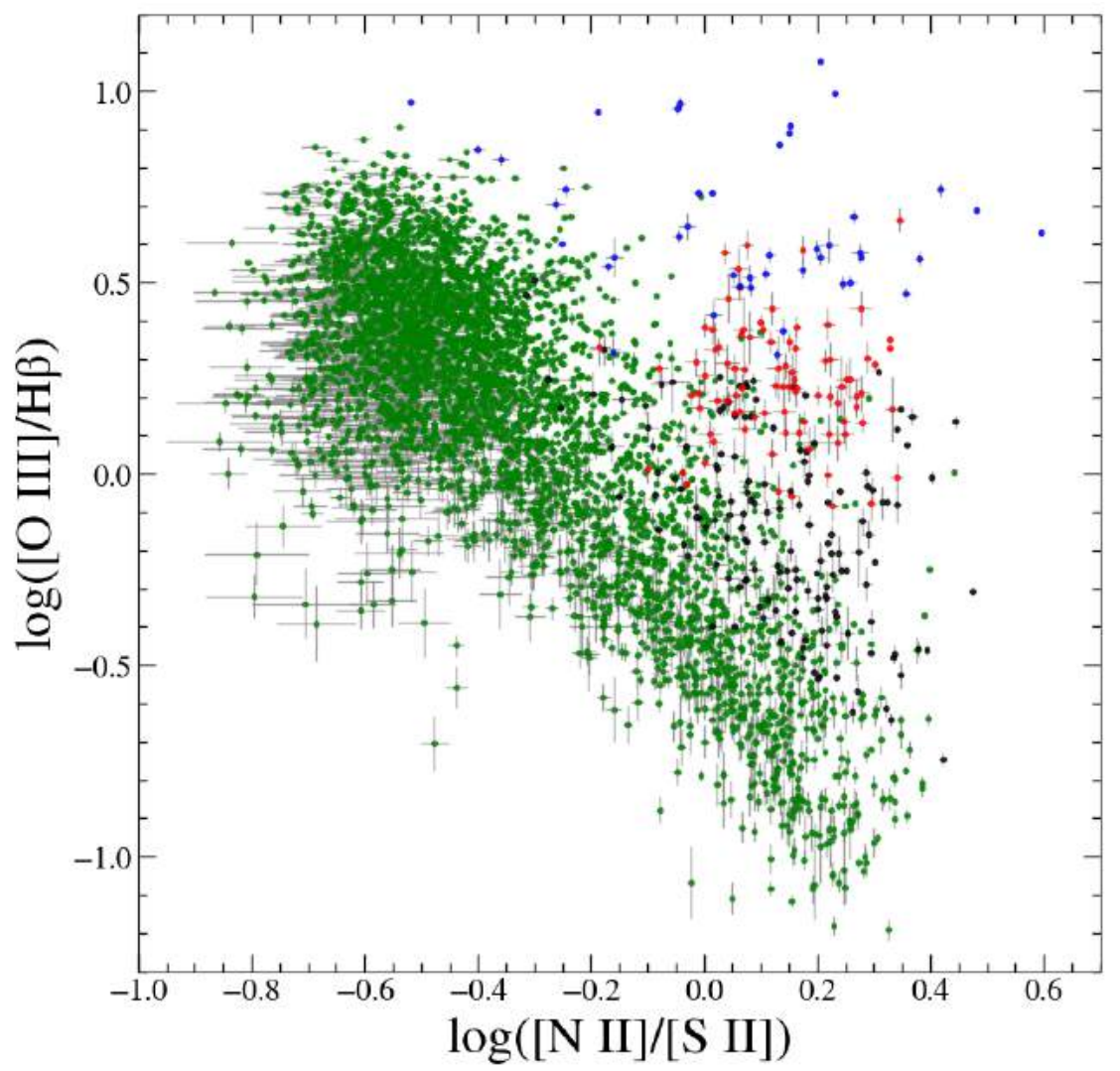

Figure 3.25: Alternative diagnostic diagram introduced by Dopita et al. (2013). Points in green are star-forming nuclei, in red are LINERs, in blue are Seyfert nuclei, and in black are transition objects, according to the lines proposed by Kewley et al. (2001) (see Equation 3.1), Kauffmann et al. (2003) (see Equation 3.4, and Cid Fernandes et al. (2010) (see Equation 3.14).

were not already addressed by the standard diagnostic diagrams, probably because they are based on the exact same line ratios. However, we have shown that they can be used to improve the way objects are classified depending on how the alternative diagrams are constructed.

For example, we tested if any of the alternative diagnostic diagrams could better identify the population associated with photoionization by hot old stars. Nevertheless, such population overlaps with LINERs just like in the standard diagrams. To solve this problem, it would be necessary to consider other features of the spectra, as already mentioned in the previous Sections. Some interesting parameters that aid the classification are the equivalent widths of emission lines (for example $\mathrm{H} \alpha$ as in the WHAN diagram of Cid Fernandes et al. 2010), the $4000 \AA$ break (as used in the DEW diagrams of Stasińska et al. 2006), the velocity dispersion (for example as in the 3D diagram for IFU data of D'Agostino et al. 2019 and in Aydar and Steiner 2016), and the radial profile of emission lines (e.g., 
Sarzi et al., 2010; Yan and Blanton, 2012, Singh et al., 2013; Belfiore et al., 2016). Other parameters can aid in the distinction between ionization by evolved stars and shocks, for example.

\subsubsection{Summary}

In summary, we have proposed four alternative diagnostic diagrams with their respective classification schemes. Three of these are new and combine all the emission-line ratios from the standard diagnostic diagrams, and one is more economic by considering just three lines in a narrow wavelength range from $\mathrm{H} \alpha$ to $[\mathrm{S} \mathrm{II}]$. The diagram based on the sum of all emission-line ratios can separate star-forming regions, Seyferts, and LINERs, as shown in Figure 3.13. The diagram based on the difference of the nitrogen and sulfur line ratios can separate star-forming nuclei and AGNs, and also to define a transition region, as in Figure 3.15. The diagram based on the difference between the oxygen line ratios can distinguish star-forming objects, Seyferts, and LINERs. We studied the theoretical separation between the two types of AGNs in Figure 3.17, but obtained better results using our own empirical classification scheme proposed in Figure 3.19. We presented a diagram that considers only the emission lines of [N II], [S II], and $\mathrm{H} \alpha$ to separate star-forming objects, Seyferts, and LINERS, according to Figure 3.21, but found it is safer to only separate objects into star-forming and AGNs as shown in Figure 3.23. Finally, in Figure 3.25 we presented an alternative diagram that is not used for diagnostics, but will be used in Section 3.3.4.

The diagnostic diagrams proposed here will be tested with theoretical models in the following Section in order for us to better understand how each parameter affects the position of an object.

\subsection{Theoretical modeling}

Theory and observations should be combined in order to produce more meaningful science. Since we are studying galactic nuclei with a phenomenological approach, we need theory in order to comprehend the physical mechanisms at play. In Section (3.2) we introduced new methods to analyze observational data based on empirical classification schemes to separate objects according to their main power source. Now, in this Section, in order to better understand the behavior of the distribution of the nuclei according to 
fundamental parameters in the alternative diagnostic diagrams, we will use theoretical tools to study the main physical parameters that determine the line ratios observed.

Several advances are being made in order to properly model the spectra of star-forming galaxies, AGNs, and shocks. However, since the main phenomena are not observed in isolation in nature, it is often necessary to model the spectra of galaxies with combinations of such excitation sources, though such models are still not totally self-consistent (Kewley et al., 2019).

The observations of emission lines produced in galactic nuclei have been frequently used in conjunction with theoretical models in order to constrain the physical structures of the ionized gas that produced such features. The use of the diagnostic diagrams, therefore, is not limited to the distinction among the possible excitation sources of these nuclei, but can also enhance the details of the physical processes that are happening by comparing the observations with the predictions of the theoretical models. In that sense, the models that best fit the observational data can indicate the physical parameters and excitation mechanisms that are most likely acting at each location of the diagrams (Groves et al. 2004). Since the alternative diagnostic diagrams were proposed based on observational constraints, it is interesting to use synthetic models to analyze which parameters are most relevant to describe our sample and how the variation of the values of each parameter can move the objects in the diagrams.

The models that describe HII region-like objects and AGNs must be different, since the sources of excitation are significantly distinct. Models have been proposed and refined since the 1960s, and they still present several caveats.

However, at least part of the basis of the modeling of gaseous nebulae is the same in all cases, as it depends on databases of atomic data that are produced considering sophisticated quantum mechanical modeling and laboratory measurements. Such databases include energy levels, collision and excitation rates, and collision cross sections, as well as data on the composition and effects of interstellar dust. Concomitantly, large scale highquality observations have provided a database of galactic spectra, and the combination of empirical and theoretical information advances our understanding of the processes that produce these spectra, including photoionization, collisional excitation, and shock physics (Kewley et al., 2019).

With regard to models of AGNs, we have models that consider purely the photoion- 
ization from the central sources (e.g., Halpern and Steiner, 1983; Stasińska et al., 2006; Feltre et al., 2016), models that consider shocks (e.g., Evans and Dopita, 1985; Dopita and Sutherland, 1995; Dors et al., 2021), and other complex models, as the combination of matter- and radiation-bounded clouds (e.g., Binette et al., 1996), locally emitting clouds (e.g., Ferguson et al., 1997), and dusty models dominated by radiation pressure (e.g., Dopita et al., 2002; Groves et al., 2004). Such complex models to describe AGNs are explained in more detail in Appendix A.

As for $\mathrm{H}$ II region-like objects, the models usually consider a combination of stellar evolutionary synthesis simulations and photoionization models. The choice of evolutionary track, stellar atmosphere, star formation rates and histories, initial mass function (IMF) 1) treatment of stellar winds, binary star evolution, and other parameters to define the SED that is ionizing the medium will drive significant differences in the modeled spectra (Kewley et al., 2001; Stasińska et al., 2006: D'Agostino et al., 2019), as further detailed in Section 3.3.1. Some relevant grids of theoretical star-forming regions have been presented by McCall et al. (1985), Leitherer and Heckman (1995), Kewley et al. (2001), Levesque et al. (2010), and many others, in order to provide calibrations and to set plausible ranges for the parameters that are relevant.

As for the parameters that apply to both AGN and H II region-like models, the most relevant are the gas phase metallicity $(Z)$, the ionization parameter $(U)$, the gas electron density $\left(\mathrm{n}_{\mathrm{e}}\right)$, and the hardness of the ionization field (Belfiore et al., 2016). This last parameter depends on the stellar SED in the case of star-forming regions, and is usually defined as a broken power-law in the case of AGNs, with specified spectral indexes $(\alpha)$. Hence, the models also need to assume an ionization structure, a temperature structure, and a density structure of the nebula (Kewley et al., 2019). All of these parameters will be further specified in Section 3.3.2, where we present the ranges of values we have chosen to represent our sample on the diagnostic diagrams.

It is important to emphasize that ionization lines of different species will probe different zones of a nebula, since they have distinct ionization potentials and critical densities. For example, lines as $[\mathrm{NeIV}],[\mathrm{OV}],[\mathrm{Ar} \mathrm{IV}],[\mathrm{NIV}]$, and $[\mathrm{Sv}]$ can indicate regions of high ionization, that will likely be closer to the ionizing source. The [O III] and C III] lines are

\footnotetext{
${ }^{1}$ Initial Mass Functions describe the number of stars per unit mass at the beginning of the formation of a stellar cluster.
} 
produced throughout the nebula, in a way that their derived properties can be considered as broadly representative of the overall nebula. Emission lines as $[\mathrm{N}$ II $]$ and $[\mathrm{O}$ II $]$ trace similar regions of the nebula, being good indicators of intermediate-zone pressures and metallicities. Species associated with lower-ionization level lines, such as [S II], [O I], and $[\mathrm{N}$ I] are tracers of the very outer edge of the nebula, indicating the partially ionized zone (Kewley et al., 2019). The many intrinsic complexities of the photoionized gaseous nebulae make these objects difficult to model with simple assumptions, and that is one of the reasons why the theoretical models are still being improved and refined to better represent them.

The models used in this work are rather simplistic, and they are important in order to give insights about the general effects of the relevant parameters to the proposed diagnostic diagrams and the limitations of assumptions when trying to model complex objects. The parameters considered in the models are described in the following Sections 3.3.1 to 3.3.3), with their application in the diagnostic diagrams in sequence (Section 3.3.4). If the models are adequate, they should be able to describe the same kind of objects in the different emission-line ratio combinations that are being proposed. We discuss the limitations of our models of photoionized nebulae in the end of this Section, and we present more complex approaches for modelling AGNs in Appendix A.

\subsubsection{Stellar spectral energy distribution}

The stellar evolutionary synthesis models were first developed by Tinsley (1968), by considering the calculation of a large stellar population with continuous star formation in bins of stellar mass that were placed in the Hertzsprung-Russell diagram and evolved according to evolutionary tracks, successfully reproducing the colors, mass-to-light ratios, relative gas masses and types of galaxies for which the rate of stellar birth and the age of the stellar population were set. These models have been developed further with the inclusion of calculations about chemical evolution, massive star evolution, stellar and gas-phase abundances, heavy element yields, changing star-formation histories, secondary nucleosynthesis, nebular emission and internal extinction, stellar mass loss, and the consideration of new observational data sets with giant branch luminosity functions, near-IR and UV stellar spectra, stars in the horizontal asymptotic giant branch and post-asymptotic giant branch, and stellar atmosphere opacities (see Kewley et al., 2019, and references therein). 
In that sense, we now have several theoretical tools that help us interpreting the spectra of galaxies. Some of the recent codes in the literature are STARBURST99 (Leitherer et al. 1999), Stochastically Lighting Up Galaxies (SLUG, Krumholz et al.2015), Projet d'Étude des GAlaxies pas Synthèse Évolutive (PÉGase, Fioc et al. 2011), Galaxev (Bruzual and Charlot, 2011), PopStar (García-Vargas et al., 2013), and Binary Population and Spectral Synthesis (BPASS, Stanway et al. 2016). Such projects are optimized to reproduce the observed properties of stellar populations and galaxies with active star formation, but given the wide choice in stellar mass loss formulations, evolutionary tracks, and stellar atmospheric transfer models, they provide different predictions of the shape and intensity of the EUV field as a function of the stellar age (Kewley et al., 2001).

In these codes, the inputs are usually related to the IMF, the SFR, the stellar atmosphere formulation, and the choice of the stellar population from an instantaneous or a continuous starburst. The main output is the synthetically generated spectrum. By combining the UV radiation field that is produced in these stellar population synthesis models with photoionization models (described in details in Section 3.3.2), we are able to generate models of $\mathrm{H}$ II regions or starbursts and therefore to simulate the spectra of galactic nuclei with ongoing star-formation. The work by D'Agostino et al. (2019) compares all the above mentioned codes except for GALAXEV and POPSTAR in order to reproduce the EUV and emission-line spectra predicted for extragalactic H II regions excited mainly by young clusters of OB stars, and finds that the main results are in agreement, with some recommendations with regard to the choice of the initial parameters that will be presented throughout this Section.

One of the conclusions from D'Agostino et al. (2019) is that Starburst99 is suitable for modelling single stellar populations (SSPs). This type of code computes the spectrum of a stellar population based on their SFH, age, and metallicity, by populating stellar evolutionary tracks with stars in agreement with the defined IMF, according to the chosen libraries of stellar atmosphere spectra. Hence, the output will be a synthetic spectrum based on the integral of these simple stellar populations with regard to the rate at which stars have formed over time.

The stellar evolutionary synthesis code used in this work is STARBURST99 Leitherer et al. 1999 2 . This code is based on an assumption of fixed metallicity to include so-

\footnotetext{
2 The models can be run at https://www.stsci.edu/science/starburst99/docs/parameters.html.
} 
phisticated treatment of stellar processes as stellar rotation, mixing metal opacities, and massive-star evolution, being very useful to model the global emission from starburst galaxies. The wavelength range covered by these models goes from $91 \AA$ to $160 \mu \mathrm{m}$.

We have chosen to use continuous star formation to represent the star-forming galactic nuclei, which means that we are considering that young stellar populations are continuously emerging and contributing to the overall spectrum. This is more appropriate to describe galaxies that contain a large number of $\mathrm{H}$ II regions of different ages, with new stellar populations emerging repeatedly (Stasińska et al., 2006; Levesque et al., 2010). The instantaneous star forming scenario is more adequate to model isolated $\mathrm{H}$ II regions, with a single stellar population dominating the ionizing spectrum (D'Agostino et al., 2019), which is not likely the case in our sample. The continuous scenario considers a balance between stellar birth and death for all the stellar masses that contribute significantly to the resulting EUV spectrum (Kewley et al., 2001). We maintained the default SFR of 1 $\mathrm{M}_{\odot} \mathrm{yr}^{-1}$, but note that our results are not dependent on the absolute SFR scaling.

With regard to the age, for the continuous star formation scenario, stellar populations older than $\sim 6.5$ Myr reach an equilibrium between birth and death of massive stars, and therefore their resulting EUV spectra become time independent (Meléndez et al., 2014). The EUV spectra will be harder for lower ages of the stellar population, since the highmass stars are mainly responsible for the EUV field in such regimes (Kewley et al., 2001). Beyond ages of $6 \mathrm{Myr}$, the fluxes of $\mathrm{H}^{0}-, \mathrm{He}^{0}$ - and $\mathrm{He}^{+}$-ionizing photons from a single stellar cluster decrease considerably (D'Agostino et al., 2019). Hence, in this work we have compared ages of $10^{6}$ and $10^{8}$ years.

Though many of the works that use synthetic stellar SEDs consider a Salpeter IMF, which is the default configuration for Starburst99 (e.g., Kewley et al., 2001; Stasińska et al., 2006), we decided to adopt the IMF of Equation 5 from Kroupa (2002), considered in more recent works as D'Agostino et al. (2019). Such IMF consists on a broken power law with three distinct segments, described by the indexes of $0.3,1.3$, and 2.3 with respect to the mass intervals of $0.01,0.08,0.5$, and $120.0 \mathrm{M}_{\odot}$. We have maintained the canonical supernova and black hole cut-off masses of 8 and $120 \mathrm{M}_{\odot}$, respectively.

The stellar evolutionary tracks consider the evolution of a star with certain initial mass from the main sequence until its end point, as a supernova or white dwarf. The interpolation of such tracks to isochrones will give the distribution of a stellar population 
with determined age and metallicity (D'Agostino et al., 2019). The chosen stellar track was Geneva standard, which is a selection of the 1994 Geneva tracks with "standard" mass-loss rates. It includes the early asymptotic-giant-branch evolution until the first thermal pulse for masses $>1.7 \mathrm{M}_{\odot}$, and the lowest mass included is equivalent to $0.8 \mathrm{M}_{\odot}$ (Leitherer et al. 1999). We have tested the Geneva tracks with high mass-loss and stellar rotation, but we decided to present only the standard models, in order to have simplistic models that are easy to interpret, though the former options provide harder ionizing spectra for the same initial parameters, with differences of $\sim 0.03$ dex in the comparison of the emission-line ratios of the mass-loss cases (Kewley et al., 2001).

We have chosen the atmosphere for the low-resolution spectrum to be according to Pauldrach/Hillier models, as recommended in D'Agostino et al. (2019). The stellar atmosphere models are important to the shape of the ionizing spectra, since the differences in the atmospheric opacities influence the intensity of the emission lines. The Hillier models (Hillier and Miller, 1998) consider WR atmospheres (including line blanketing), whereas the Pauldrach models (Pauldrach et al., 2001) account for OB atmospheres (including updated metal opacities, an improved atomic data archive, and revised EUV and X-ray radiation models from the shock cooling zones in the OB stellar winds).

The remaining configurations that were not specified in this Section were kept at the default values of Starburst99. In Figure 3.26 we show two Starburst99 models we generated, from $200 \AA$ to $1 \mu \mathrm{m}$ in wavelength. The blue spectrum represents our lowest metallicity model and the red spectrum refers to the highest considered metallicity.

We have run models with all the available metallicities, which are $0.2,0.4,0.7,1.0$, and $2.0 \mathrm{Z}_{\odot}$. These values are limited to the stellar tracks and opacity tables at hand. In Figure 3.27, we compare the spectra of each metallicity, by normalizing them with regard to the solar metallicity spectrum, and we can notice that the differences between them are up to several orders of magnitude for the lowest wavelengths. In this graph, we are showing the wavelength range from 100 to $1000 \AA$, which is the interval where the spectra are significantly distinct. We can see that the metallicities smaller than solar will present more energetic photons, while the metallicity that is twice the solar abundance will present mostly lower luminosities in this wavelength range, as already seen in Figure 3.26 .

We emphasize that we have not considered binary stellar populations. D'Agostino et al. (2019) shows that, though binary populations can produce spectra that are harder 


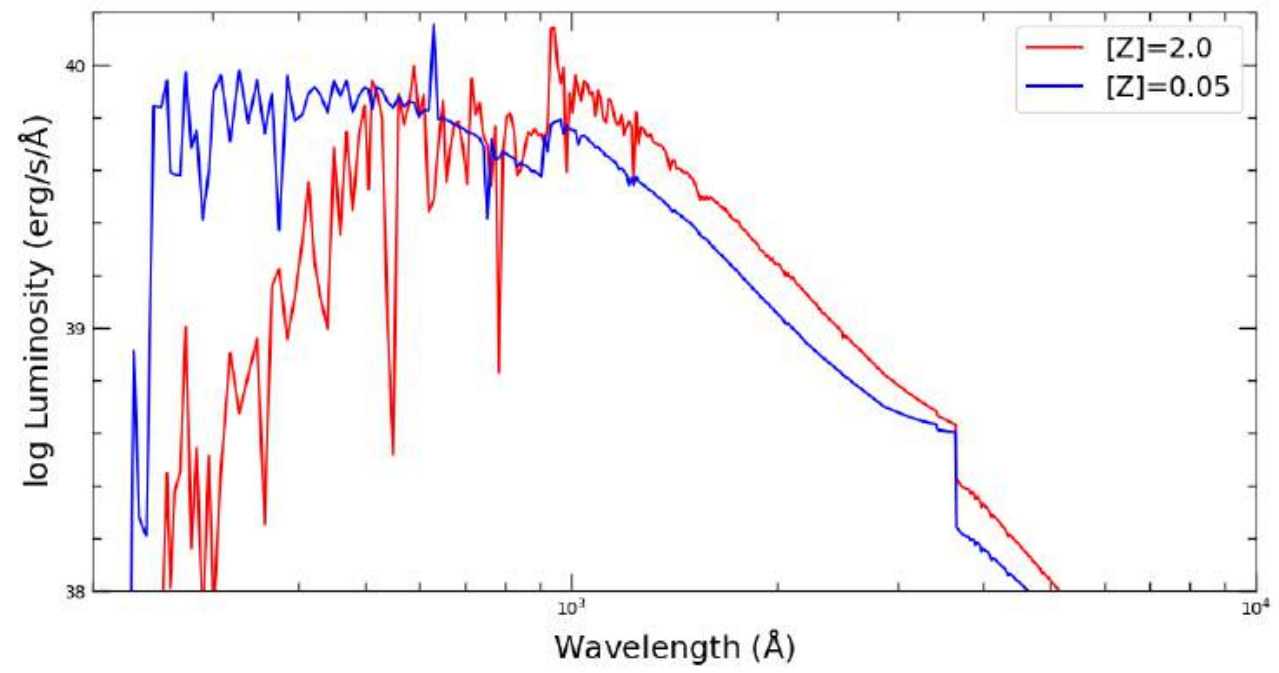

Figure 3.26: Two examples of synthetic spectra of the stellar populations produced with STARBurst99. The blue spectrum refers to the metallicity of $0.05 \mathrm{Z}_{\odot}$ and the red spectrum refers to $2.0 \mathrm{Z}_{\odot}$.

than SSPs, the differences in the emission-line ratios of the two procedures is rather small. STARBURST99 models do not to include binary evolution because, although $50 \%$ of all stars are formed in binary systems, it is not clear if a significant fraction on such binaries will present an evolutionary history that differs substantially from single-star evolution. Hence, facing the polemics about such topic, it was decided to implement the simpler approach.

Regarding WR stars, it is crucial to consider them in the H II region-like nuclei, since these objects play an important role in determining the intensity and the shape of the EUV spectrum. However, the modeling of such stars still presents uncertainties about their lifetimes, their wind mass-loss rates, the velocity law of their stellar winds, and their atmospheric opacities (Kewley et al., 2001). The presence of WR stars is more relevant at higher ages and metallicities. The relevance of WR stars affects our choice for the Geneva tracks instead of the Padova tracks that are also available at StARBURST99 code.

As for the remnants of supernovae, Kewley et al. (2019) concludes that their contribution to the emission-line ratios can be neglected, though they do affect the resulting spectra, similarly to the case of binary systems.

D'Agostino et al. (2019) finds that the variations in the parameters associated with the ionizing radiation field (such as SSP codes, stellar atmospheres, stellar evolutionary tracks, and IMF) are small, with an average systematic difference of $\sim 0.1$ dex in the optical emission-line ratios between the varying models. Hence, we have chosen simple models in 


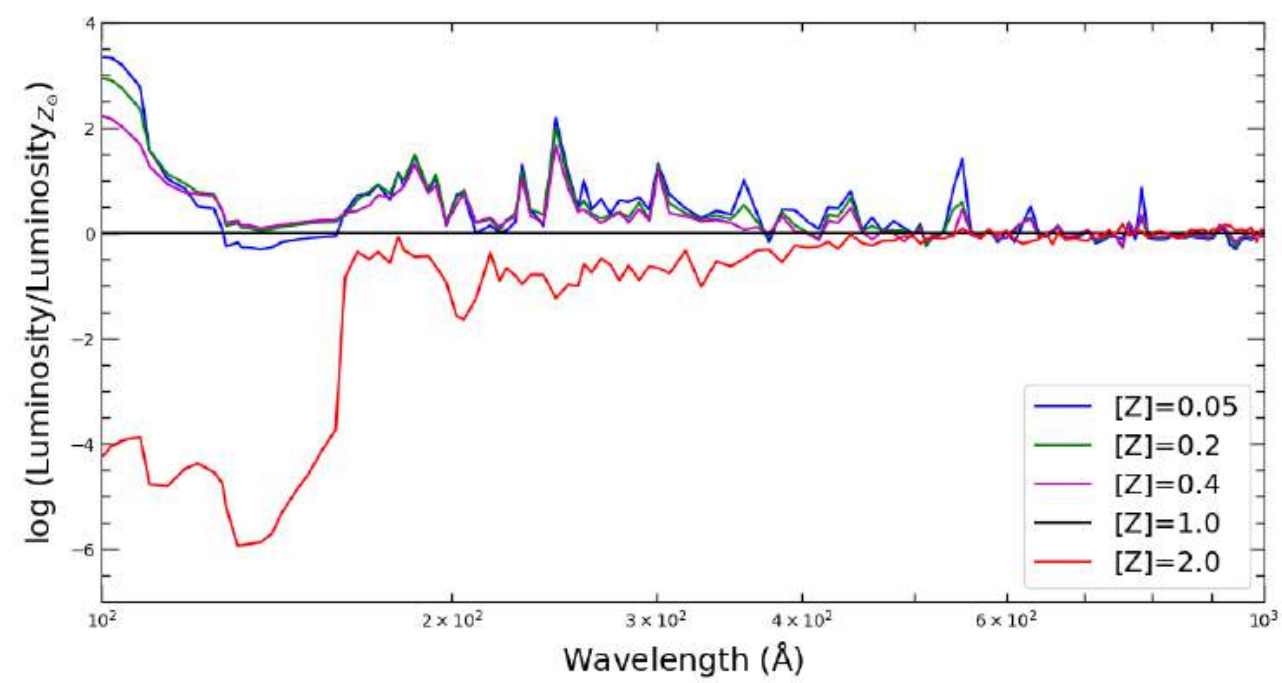

Figure 3.27: All synthetic spectra of the stellar populations produced with STARBURST99 for this work. The blue spectrum refers to the metallicity of $0.05\left(\mathrm{Z} / \mathrm{Z}_{\odot}\right)$, the green spectrum refers to $0.2\left(\mathrm{Z} / \mathrm{Z}_{\odot}\right)$, the magenta spectrum refers to $0.4\left(\mathrm{Z} / \mathrm{Z}_{\odot}\right)$, the black line is the reference of normalization, which is the solar metallicity, and the red spectrum refers to $2.0\left(\mathrm{Z} / \mathrm{Z}_{\odot}\right)$.

order to analyze our sample in relation to the standard and alternative diagnostic diagrams.

\subsubsection{Photoionization models}

The physical conditions of photoionized nebulae are extremely complex, in a way that it is not possible to use analytical solutions to describe them. Instead, we rely on photoionization models to better understand the mechanisms in such kind of objects. Historically, the $\mathrm{H}$ II region and the AGN photoionization models were developed separately (Kewley et al., 2019).

Regarding H II regions, Strömgren (1938) proposes to represent them with models of spheres of gas, that with some modifications are still in use today. Zanstra (1951) proposes the "on-the-spot" approximation, showing that the ionization state of the gas can be calculated if the gas is optically thick by considering that the recombination of ions with electrons to the ground state produces diffuse radiation that immediately causes reionization of the neutral gas. Williams (1967) considers the thermal balance and ionization within the gas that surrounds a diffuse ionizing radiation field, while Rubin (1968) considers thermal and ionization equilibrium with an arbitrary density distribution. The advances in the models of stellar tracks and atmospheres were presented in Section 3.3.1, and further details can be found in the review by Kewley et al. (2019). Grids of photoionization models that reproduce the star-forming sequence are presented in Dopita and 
Evans (1986), Dopita et al. (2000), Kewley et al. (2001), Dopita et al. (2006), Nagao et al. (2006), Stasińska et al. (2006, 2008), Gutkin et al. (2016), and many others.

As for the AGNs, the first models were used to calculate the temperatures and densities in quasars, as in Osterbrock and Parker (1966) and Mathez (1968). The inclusion of stratified models for different zones to contain ions at distinct stages of ionization is given in Burbidge et al. (1966) and Bahcall and Kozlovsky (1969ba). The effects of X-ray radiation in optically thick and thin clouds (Tarter et al., 1969; Tarter and Salpeter, 1969), and Auger ionization, Compton heating, and charge transfer collisions (Hatchett et al., 1976; Halpern and Grindlay, 1980) have since been included. Davidson (1972) proposes photoionization models with iterative calculation, where the gas has a spherical symmetry and a quasar is the central source. High-temperature shocks are introduced by MacAlpine (1974) as a possible cause of the observed forbidden lines, whereas Shields (1974) shows that a powerlaw radiation field is able to explain the emission from the Seyfert 1 galaxy 3C 120. The effects of resonance line-trapping and collisional de-excitation to the emission-line spectra of high density gas are included in models by Krolik and McKee (1978), Ferland and Netzer (1979), and Kallman and McCray (1982).

At present, there are two main photoionization codes that include all the topics mentioned above and that received regular updates. MAPPINGS was developed by Binette (1985) and Sutherland and Dopita (1993, 2017). It includes self-consistent treatment of nebular, dust, and shock physics in such a way to be applicable to H II regions, AGNs, and it can also separately simulate regions shocked by supernovae, galactic winds, and jets (Kewley et al., 2019). The other code is CLOudy, developed by Ferland et al. (1998, 2013, 2017). This code includes self-consistent treatment of nebular, dust, and molecular physics that can be applied to HII regions, AGNs, and photodissociation regions. If we set a stellar SED in CLOUdy, as detailed in Section 3.3.1, we can simulate an H II region or a starburst galaxy. The ideal models couple the stellar continuum and nebular gas to self-consistently reproduce the line intensities that scale with the stellar population with regard to age and metallicity (Byler et al., 2017). Last, if we set a power-law spectrum (whether simple or broken), we can reproduce an AGN, as shown for example by Ferland and Netzer (1983), Halpern and Steiner (1983), Stasińska (1984), Ferland and Osterbrock (1986), Storchi-Bergmann et al. (1998), Groves et al. (2006), Stasińska et al. (2006), Feltre et al. (2016), Castro et al. (2017), Pérez-Montero et al. (2019), Carvalho et al. (2020), and 
many others. Shocks might affect the intensity and shape of the emission lines, but they are usually not the main drivers of the ionization in AGNs (see Dors et al., 2021, for cases where shock dominates over photoionization), so photoionization from the central source has to be included in the models. It is interesting to consider both shocks and photoionization by a power-law simultaneously, as in Dors et al. (2021); we go into further details about shock models on Appendix A.3. The techniques for modeling AGN ionizing radiation fields are still being developed, with examples as in Groves et al. (2004) and Thomas et al. (2016). Therefore, spectroscopical analyzes of the intensity of emission-line ratios that rely on photoionization models have proven to be an optimal way of interpreting the features of different ionizing sources in the nuclei of galaxies (Feltre et al., 2016).

A photoionization model, whether describing the nebular emission of a star-forming region or an AGN, is parametrized mainly by the shape and luminosity of the ionizing continuum, the density profile of the gas, the chemical composition of the gas, and the ionization parameter (Ferland and Osterbrock, 1986; Stasińska et al., 2008). Since emission-line ratios can be used to obtain observational estimates of the parameters, it is evident that the choice of inputs will affect directly the theoretical prediction of the lines.

The main difference between the nebular emission of star-forming nuclei and AGN is due to the spectral energy distribution of the incident ionizing radiation, as can be seen in Figure 1 from Feltre, Charlot \& Gutkin (2016), reproduced here in Figure 3.28. Comparing the stellar SEDs, we note the effects of the metallicity, since the two stellar population spectra differ significantly for photon energies above $20 \mathrm{eV}$. The lowest-metallicity present a harder radiation field since low-metallicity stars evolve at higher temperatures and luminosities in comparison to high-metallicity populations (Chen et al., 2015). As for the comparison of the stellar and AGN SEDs, we see they are relatively similar until $\sim 30$ $\mathrm{eV}$, while for energies greater than $\sim 50 \mathrm{eV}$, the spectra associated with accretion disks of SMBHs have significant flux while the stellar spectra abruptly drop. Therefore, emission lines associated with ion species that require ionization energies above $50 \mathrm{eV}$ are promising indicators of the presence of an AGN, since they are absent in the stellar spectra.

Other differences to consider when modelling AGN and star-forming regions is that low-metallicity AGN are extremely rare in the Local Universe (Groves et al., 2006), but low-metallicity star-forming galaxies are common; and also that the observed densities in the gaseous nebulae, measured from optical line-doublets, are usually higher for AGNs 


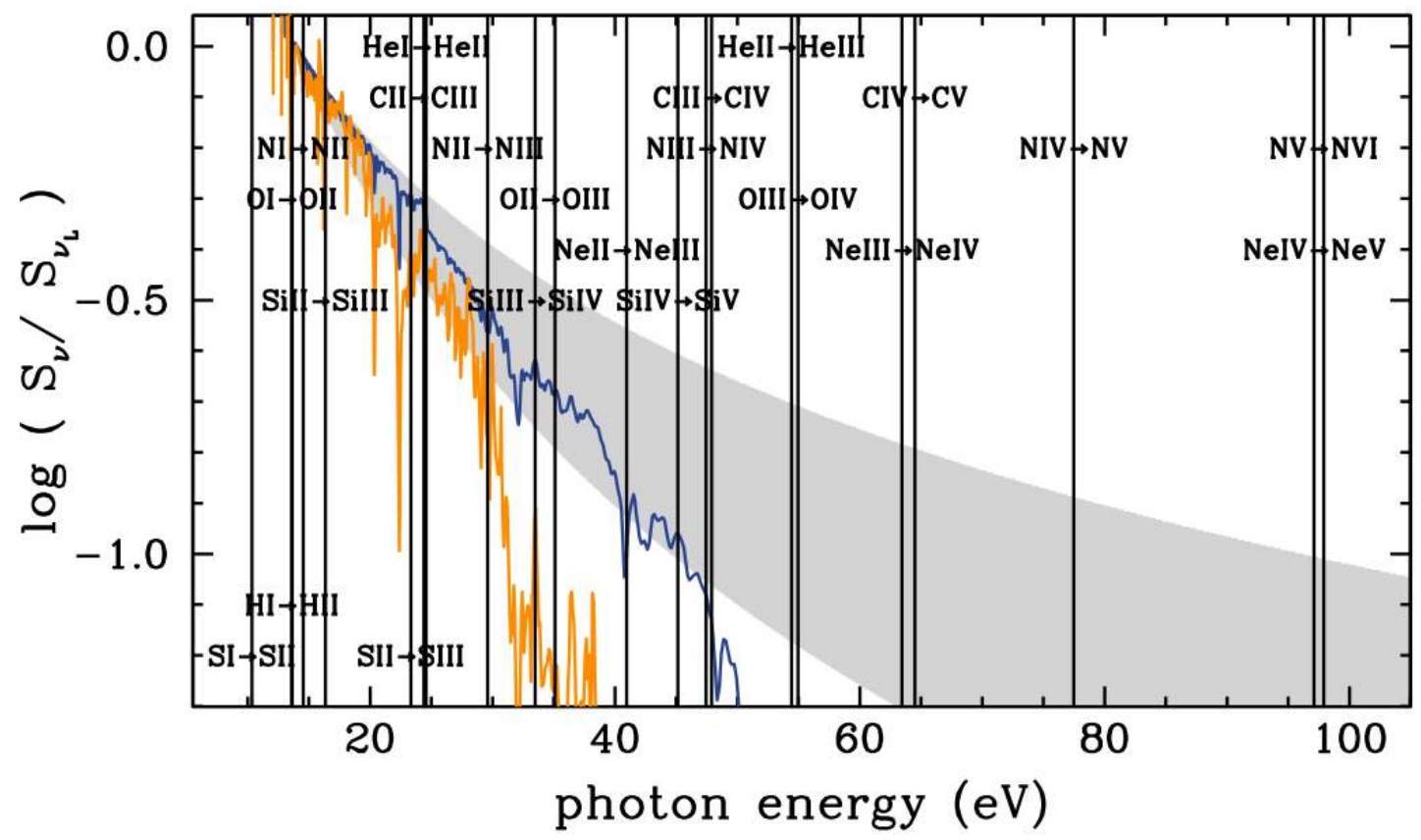

Figure 3.28: Figure 1 from Feltre, Charlot \& Gutkin 2016), showing examples of spectral energy distributions (in units of the luminosity per unit frequency at the Lyman limit) of the incident ionizing radiation in the AGN and star-forming models proposed in the work. The gray shaded area indicated the location of AGN ionizing spectra with power-law indices between $\alpha=-2.0$ (bottom edge) and -1.2 (top edge). The blue and orange lines show the ionizing spectra of stellar populations of metallicities $[Z]=0.05$ and 1.5, respectively. Vertical lines indicate the ionizing energies of ions of different species.

than for H II regions (Osterbrock and Ferland, 2006; Feltre et al., 2016).

The relation between the metallicity and the ionization parameter for star-forming regions is debated extensively, with several disagreements between different methods. Theoretically, the ionization parameter should be dependent on the metallicity because the stellar atmospheres of massive O stars become cooler for higher abundances, decreasing the ionization parameter. Moreover, the stellar atmospheres of objects with higher metallicity scatter photons more efficiently, causing a greater conversion of luminous to mechanical energy flux in the stellar wind base region, that would also affect the ionization parameter by decreasing it (Dopita et al., 2006; Dors et al., 2011). Some authors including Dopita and Evans (1986), Bresolin et al. (1999), Brinchmann et al. (2004), Maier et al. (2006), and Nagao et al. (2006) argue that there is a relation between these parameters for star-forming objects, while others including Garnett et al. (1997), Kennicutt and Garnett (1996), Dors and Copetti (2005), Dors et al. (2011), and Poetrodjojo et al. (2018) argue there is no relation at all or that this relation is too weak to be significant.

We have opted to use Cloudy photoionization code instead of MAPPINGS. These 
codes present significant disagreement between the general spectra generated based on the same metallicities and ionization parameters, mainly due to the intrinsic differences related to atomic data sets, input physics, and model assumptions. However, the overall difference in the resulting emission-line ratios is only $\sim 0.1$ dex on average (D'Agostino et al., 2019).

Cloudy ${ }^{3}$ is a code that simulates the ionization, chemical, and thermal states of a material exposed to an external radiation field or other source of heating, and it predicts observables such as emission and absorption spectra (Ferland et al., 2013). The input parameters are the geometry (spherical or plane-parallel), the composition (with regard to the ionization, the chemical abundance, and the dust), the density, the thickness of the cloud, and the radiation field that hits the nebula. The output is the spectrum, with thermal, ionization and chemical properties associated.

Considering the SEDs of Starburst99 and the photoionized nebula of Cloudy, we have run several models, in some cases drawing model grids over the data and in other cases simply choosing the models that showed the best mean fit. The next paragraphs of this section will describe each of the parameters and the justifications for the choice of the used values for density, ionization parameter, geometry, SED, metallicity, and temperature.

Density: There are two relevant densities to consider when treating a gaseous nebula, which are the electron and the hydrogen density. The electron density $\left(n_{e}\right)$ is given by the number of electrons per unit volume in a region, in number of electrons per cubic centimeter. The hydrogen gas density $\left(n_{\mathrm{H}}\right)$ is analogous, but with regard to the sum of ionized, neutral and molecular hydrogen. The electron and ionized hydrogen densities will be approximately the same in a fully ionized medium (Belfiore et al., 2016), but they might differ in regions such as the partially ionized zone, where we expect to have more neutral hydrogen atoms than free electrons.

Ions have specific energy levels and thus are sensitive to different density regimes, depending on the critical density of the considered transition. Lines like [S II] and [O II] that have low critical densities will be affected by collisional de-excitation, being weak in high-density regions. Ions like [Cl III] and [Ar IV] are good tracers of higher densities, giving information about the pressure conditions of the ISM. [O III] has a critical density between

\footnotetext{
3 The open source software is available at https://nublado.org/, and the founder of the code Gary Ferland is keen to answer questions and solve problems.
} 
the two types of ions mentioned, and it will trace regions with densities lower than the zones where [Cl III] and [Ar IV] are emitted (Kewley et al., 2019).

To determine the density of a region, we use doublets of emission lines from the same ion species in which the levels have different collisional strengths. In the optical domain, the most widely used doublets are [S II] $] \lambda$ 6716,31 and [O II] $] \lambda \lambda 3726,28$ Osterbrock and Ferland, 2006). It is worth pointing out that, if we are observing a clumpy region with integrated spectra, the line emission will effectively trace the density of the clumps instead of a global average density (Belfiore et al., 2016).

The density of a nebula is commonly described by four model spatial structures: a constant distribution, a radial profile, filaments, or clumps. The simplest consists of a single density. This approach is often considered because of its simplicity, but is not very accurate for the complex and inhomogeneous structures that we see at high spatial resolution (e.g., Kakkad et al., 2018; Kewley et al., 2019). Other issues with this toy model is that lines of different species usually present different widths and also that the density measured using different line doublets (for example [S II] $\lambda 6716 / \lambda 6731$ and [O II $] \lambda 3729 / \lambda 3726$ ) are incompatible with a constant density.

Several works have tried to characterize the density distribution of a gaseous photoionized nebula based on measurements along their radius. Some results present complex radial gradients (e.g., Franco et al., 2000; Pérez et al., 2001; Binette et al., 2002; Luridiana and Cerviño, 2003; McLeod et al., 2016), while others reveal relatively shallow gradients (e.g., Ramos-Larios et al., 2010; García-Benito et al., 2010). Some works measure a $r^{-1 / 2}$ profile for the gas distribution from the center of the galaxy (Gutiérrez and Beckman, 2010; Revalski et al., 2018), while other works have found that the presence of bars (Herrera-Camus et al. 2016) and galactic-scale winds (Ho et al., 2014; Rose et al., 2018) also affect the density distribution. Fortunately, Dors et al. (2019) shows that photoionization models with different radial density profiles will produce emission line intensities similar to the simplest models of constant density.

The third possible geometry considers gas condensed into small filaments. Such structures would be optically thin to the ionizing continuum, and would lie over a large range of radii (Ferland and Osterbrock, 1986).

The last option for the density configuration is the most accurate and also the hardest to model, which is the distribution of the gas in small optically thick clumps (see Figure 6 
of Osterbrock, 1984, for a schematic example). In this case, the relative line ratios will vary according to where they are produced, such that the density, the dimensions of each cloud, their distance to the central source, and possible shadowing effects influence the resulting emission lines (Ferland and Osterbrock, 1986). Lines with higher critical densities will be emitted mainly by high density clouds, that are more likely closer to the nucleus and present higher velocities as a consequence (Yan and Blanton, 2012).

If a flat density profile is assumed, equal weight will be assigned to all emitting regions, while, in reality, the emission is very localized in the nebula. In the cases where density gradients or clumpiness are expected, it is recommended to use models with pressure equilibrium in order to include complex density gradients and profiles (Gutiérrez and Beckman. 2010). The pressure distribution of an ISM depends on the density and temperature distributions, and it is determined by the the mechanical energy produced by the power source and the strength and shape of the radiation field (Kewley et al., 2019). Higher pressure will produce larger BPT line ratios, especially for objects with higher metallicity, where the nebula is more susceptible to the effects of cooling. According to D'Agostino et al. (2019), all galaxies in the SDSS star-forming sequence can be explained by a pressure range of $6 \lesssim \log (P / k) \lesssim 7$, where $k$ is the Boltzmann constant.

One way of incorporating clumps in the nebula is through the filling factor $(\epsilon)$, defined as the fraction of the total volume occupied by the condensations. The space in between would be vacuum, and thus does not make any contribution to the emission, mass or opacity of the nebula. The typical range of such factor is from $1 \%$ to $50 \%$ (Osterbrock and Ferland, 2006).

The density distribution of gaseous nebulae probably varies depending on the objects (Zhang et al., 2013; Dors et al., 2014). For simplicity, we adopt a constant density, and the consequences and limitations of such choice will be analyzed further in this Section. We have considered a density of $n_{\mathrm{H}}=200 \mathrm{~cm}^{-3}$ for $\mathrm{H}$ II regions (according to the values found by Copetti et al. 2000 and Kashino et al. (2017), and adopted in the models of Dors et al. 2011) and $n_{\mathrm{H}}=500 \mathrm{~cm}^{-3}$ for the NLRs of AGNs (according to the values found by Dors et al. 2014 and Nagao et al. 2006, and adopted in the models of Castro et al. 2017 and Dors et al. 2019). In order to consider the small clumps with constant density, we have chosen the filling factor to be of $1 \%$. 
Ionization parameter: The ionization parameter $(U)$ is an estimate of the amount of ionization that a radiation field can drive as it moves through the nebula (Kewley et al., 2006). It encodes information about the relative geometry of the gas and its ionizing source, and is defined as

$$
U(r)=\frac{Q(\mathrm{H})}{4 \pi r^{2} n_{\mathrm{H}} c}
$$

where $r$ is the distance from the central ionizing source, $Q(\mathrm{H})$ is the number of incident photons capable of ionizing hydrogen (energies above $13.6 \mathrm{eV}$ ) emitted per second, $n_{H}$ is the hydrogen density described above and $c$ is the speed of light (Osterbrock and Ferland, 2006). Note that the ionization parameter as in Equation 3.30 is dimensionless, as it is the ratio between the number density of hydrogen-ionizing photons and the number density of the total hydrogen gas. It is worth pointing out that the ionization parameter has a direct dependence on the density, such that, if the spatial distribution of the gas is not modeled correctly, then one should not expect the ionization parameter to be precise either ( $\mathrm{Ho}$, 2008).

For each atomic species, the number of ionizing photons at each of their ionization potentials will determine the ionization structure of the nebula. The ionization parameter is usually measured by the comparison of two emission lines from the same atomic species that are in different ionization states, which is one of the motivations behind the alternative diagnostic diagram based on the difference between the neutral and twice ionized oxygen line ratios (see Figure 3.19). Other species such as carbon, sulfur, silicon, neon, and nitrogen can also be used to determine the ionization parameter. The most sensitive diagnostics will be those that consider emission lines with the largest difference in ionization potentials, and ideally at a similar wavelength for accurate flux calibration and extinction correction (Kewley et al., 2019).

It is expected that Seyferts will have higher ionization parameters than LINERs, by definition, and also than star-forming galaxies (Kewley et al., 2006). However, in our models we did not consider this intrinsic difference between Seyferts and LINERs, but we ran a range of ionization parameters. The ionization parameter can be judged by the relative strengths of the oxygen lines or by the line ratio $[\mathrm{O}$ III $] / \mathrm{H} \beta$. We have seen in Section 3.1.1 that Ho, Filippenko \& Sargent (1997) proposes a borderline at [O III] $/ \mathrm{H} \beta \sim 3$ (see Figure 3.3), though stressing that such boundary line has no strict physical significance. As it can be inferred from large samples of NLRs of AGNs, the ionization parameter seems to 
span a wide and apparently continuous range (e.g., Veilleux and Osterbrock, 1987; Feltre et al., 2016), though it is possible to delineate the two classes of Seyferts and LINERs in distinct loci of the optical diagrams (Kewley et al., 2006), with the division in the ionization parameter set as $-1.0 \lesssim \log \mathrm{U} \lesssim-3.5$ for the Seyfert 2 galaxies and $\log \mathrm{U} \lesssim-3.5$ for LINERs (Veilleux and Osterbrock, 1987). Regarding H II nuclei, the ionization parameter can also span a wide range, depending on the metallicity of such objects (Ho, 2008), and it is typically in the range of $-3.2<\log \mathrm{U}<-2.9$ for local $\mathrm{H}$ II regions (Dopita et al., 2000) and star-forming galaxies (Moustakas et al., 2010; Poetrodjojo et al., 2018).

Based on the above results and on the grids proposed by Castro et al. (2017), we have run models with $-4.0<\log \mathrm{U}<-1.0$ in steps of 0.5 dex for both AGNs and star-forming nuclei, and removed the models that did not result in realistic positions of the diagnostic diagrams.

Geometry: The geometry of CLOUDY photoionization models can be either spherical or plane-parallel. The difference consists on a comparison between the inner radius $\left(r_{i n}\right)$ and the Strömgren radius $\left(R_{S}\right.$, see Equation 3 from Feltre, Charlot \& Gutkin 2016): if $r_{i n} \ll R_{S}$, the geometry is spherical and the thickness of the ionized region reaches the order of magnitude of $R_{S}$, while if $R_{\text {in }} \gtrsim R_{S}$, the geometry will be plane-parallel. In a more physical approach, the geometry of a photoionized gaseous nebula is determined by the position of the radiation source relative to the cloud that it ionizes. Hence, a spherical approximation can be made in cases where the ionizing source is found within the cloud, while a plane-parallel geometry is more adequate to describe cases when the ionizing source is external to the molecular cloud that it ionizes (D'Agostino et al., 2019).

In practice, maintaining the same distance from the central source for spherical and plane-parallel models will preserve the same ionization parameter, and thus the different models produce very similar spectra when all the other parameters are maintained equal (Dopita et al., 2000, Kewley et al., 2019). Also, based on the discussion of the complexity of the density distributions, the simplistic models are limited, though they are still useful for a first crude theoretical estimate.

Another important factor is the distinction between closed and open geometries. The first applies to cases where all the ionizing radiation is incident on the cloud, while the latter refers to cases where a fraction of the ionizing radiation is lost to the ISM (D'Agostino 
et al., 2019).

We have adopted the simplest option, which are plane-parallel open geometries for all cases.

SED: The main difference between the models of star-forming nuclei and AGNs is with regard to the Spectral Energy Distribution.

For H II region-like objects, the EUV radiation field is produced by the stellar evolution synthesis models described in Section 3.3.1.

Non obscured AGNs can be treated as pointlike sources represented by power-law spectra at optical and UV wavelengths (Koski, 1978; Ho, 2008), thus the spectral index $(\alpha)$ is an important parameter. This index controls the relative proportions of photons capable of ionizing different elements, as seen in the gray-shaded area in Figure 3.28 (Feltre et al. 2016$)$. We explicitly show the equation of the flux density $\left(F_{\nu}\right)$ in frequency units $(\nu)$ because $\alpha$ is sometimes defined as negative and sometimes as positive (with a minus appearing in front of the index):

$$
F_{\nu} \propto \nu^{\alpha}
$$

The hardness of the ionizing radiation field increases as a function of the distance from the star-forming sequence in the standard diagnostic diagrams (Kewley et al., 2006). That is expected because, as the spectral index becomes smaller, there will be an increase in photons that are able to ionize higher ionization species in comparison to hydrogen, as well as the ionization by X-ray photons becoming more relevant, and therefore all the emission line ratios used in the standard diagnostic diagrams will increase (Stasińska, 1984; Groves et al., 2004). The explanation for LINERs presenting harder ionizing spectra than Seyferts (since the low-ionization AGNs reach loci more to the right than the high-ionization AGNs) is based on the fact that the gas loses little energy through radiation in the case of RIAF models as in LINERs, and therefore the gas is extremely hot and the weak spectrum it produces will be harder than that produced in the high state, which produces a strong temperature gradient (Esin et al., 1997).

The power-law index of the ionizing spectra of AGNs is in the range $-2.0 \lesssim \alpha \lesssim-0.5$ (Groves et al., 2004; Ho, 2008; Miller et al., 2011), though the most extreme values are less typical (see Figure 1 from Dors et al. 2019) and a mean value is often set as $\alpha \sim-1.4$ (Zamorani et al., 1981; Ferland and Osterbrock, 1986; Miller et al., 2011). In our models, 
we considered the spectral index of the low-energy slope of the Big Bump continuum to be fixed as $\alpha(\mathrm{UV})=-1.0$. The parameter that we varied refers to the UV to X-ray spectral slope, and we used values of $\alpha(\mathrm{OX})=-1.4$ to -0.8 in steps of 0.2 .

Another important parameter that is considered within the SED specifications is the temperature. In photoionization models, the electron temperature depends on the distance to the power source. At metallicities $[Z] \gtrsim Z_{\odot}$, it is expected that the nebula will be hotter toward the outer edges, because the soft ionizing photons are absorbed by metals closer to the ionizing source. Meanwhile, hard ionizing photons reach the further regions, yielding more heat per ionization. Another relevant factor is that the ion [O III], which dominates the cooling effects in the inner nebula zone, is not very abundant in the outer nebula, where we find other ions and even neutral oxygen atoms in higher abundance since they are further away from the ionizing source (Kewley et al., 2019).

We have already explained the main effect that alters the temperature in a gaseous nebula, which is the cooling from metals, that can be altered by their depletion onto dust grains. The cooling mechanisms in AGNs and star-forming nuclei are collisions, recombination, and free-free interactions dissipating the kinetic energy of the electron gas in the form of electromagnetic radiation (Flury and Moran, 2020). The elements considered in the standard diagnostic diagrams (oxygen, nitrogen, and sulphur) are the main contributors to the cooling provided by metals in nebular gas (Dors et al., 2021). In AGNs, the high electron velocities reduce the rate of recombination, in such a way that the recombination rate is approximately proportional to the inverse of the electron temperature (Osterbrock and Ferland, 2006), and the gas is prevented from dissipating the increased thermal energy (Groves et al., 2004).

There are also many physical mechanisms that drive temperature gradients, such as hardening of the radiation field through radiative transfer effects and through the appearance or disappearance of important coolant ions, or through suppression of cooling by collisional de-excitation (Dopita et al., 2013). A harder ionizing radiation field will lead to a higher average of nebular temperature. Some of the consequences of increasing the temperature is the increase in the collisional rate of ions, which means an increase in the fluxes of collisionally excited lines such as [O III] and [N II] (D'Agostino et al., 2019). There can be some small-scale temperature fluctuations as well, that are explained by turbulent heating or shocks, and this microphysics is not currently included in the photoionization 
codes (Dopita et al. 2013).

Our considerations with regard to the temperature are implicit in the treatment of the metal abundance, the dust content, and the shape and intensity of the SEDs. The only explicit specification of the temperature is with regard to the power-law spectra of the AGNs, where we set a temperature for the big blue bump of $1.5 \times 10^{5} \mathrm{~K}$, according to the example in the CLOUDY documentation.

Metallicity: The chemical abundance is a crucial parameter for the positions these objects assume in the diagnostic diagrams. Here, we must distinguish between the stellar and nebular metallicities: the former refers to the average metallicity of the stellar population and depends on the metallicity of the ISM at the time those stars were born, while the latter is the present-day metallicity of the ISM. This property encodes information about the history with regard to star formation, mass infall, and radial mixing driven by viscous processes in galactic disks (Dopita et al. 2013$)$.

The gas-phase metallicity is often expressed in terms of the oxygen abundance relative to hydrogen, in units of $12+\log (\mathrm{O} / \mathrm{H})$. Oxygen is used as the chemical element that defines the overall metallicity mainly because it is readily observable in the optical spectrum, with their most abundant ions presenting strong emission lines (Kewley et al., 2019). We are converting the $[Z]=\left(Z / \mathrm{Z}_{\odot}\right)$ values in $\mathrm{O} / \mathrm{H}$ abundances assuming

$$
12+\log (\mathrm{O} / \mathrm{H})=12+\log \left[\left(Z / \mathrm{Z}_{\odot}\right) \times 10^{\log (\mathrm{O} / \mathrm{H}) \odot}\right]
$$

where $\log (\mathrm{O} / \mathrm{H})_{\odot}=-3.31$ or $12+\log (\mathrm{O} / \mathrm{H})_{\odot}=8.69$ (Allende Prieto et al., 2001), the same value adopted by CLOUDY 17 . However, the solar metallicity stellar populations generated with StARBURST99 for the Geneva evolutionary tracks consider an older solar oxygen abundance value of $\log (\mathrm{O} / \mathrm{H})_{\odot}=-3.13$ or $12+\log (\mathrm{O} / \mathrm{H})_{\odot}=8.87($ Grevesse and Sauval, 1998). As a consequence of this imperfect match between the gas and the stellar metallicity, the computed stellar UV photon field will be slightly softer (Dopita et al., 2006, 2013; Dors et al., 2011).

There are several methods for estimating the metallicity based on emission lines from the UV through the IR: using the direct method, the strong- and recombination-line method, empirical calibrations, or photoionization models (see Kewley et al. 2019, and references therein). The collisionally excited emission lines are sensitive to the abundance 
of their element and to the electron temperature of the gas. Since metals act as coolants in a nebula, the electron temperature is sensitive to the total gas-phase metal abundance. Therefore, the estimation of the electron temperature can provide a measurement of the metal abundance, and this is the so called $T_{\mathrm{e}}$-method or direct method, where usually the ratio of auroral oxygen lines $[\mathrm{O}$ III] $(\lambda 4959+\lambda 5007) / \lambda 4363$ is considered (Pagel et al., 1979; Alloin et al., 1979; Dors et al., 2011; Pérez-Montero, 2017). In cases of unobserved ions of the same species (that might reside in infrared or ultraviolet wavelengths that are not available for a certain object), an ionization correction factor (ICF) must be used (Dopita et al., 2013). Other strong auroral forbidden emission-lines can also be used, with recombination lines in their ratios to indicate the abundance of such element with regard to hydrogen.

The recombination-line method uses recombination lines mainly from hydrogen and helium, and in some cases where the lines are strong enough, oxygen and carbon recombination lines can also be considered. Such lines are proportional to the electron density and inversely proportional to the electron temperature, but they are not ideal in the extreme high- and low-abundances (Dopita et al., 2013). The most widely used combinations of strong emission-lines and recombination lines are $R_{2}=[\mathrm{O}$ II $] \lambda \lambda 3726,9 / \mathrm{H} \beta, R_{3}=[\mathrm{O}$ III $]$ $\lambda 5007 / \mathrm{H} \beta, R_{23}=R_{2}+R_{3}($ Pagel et al., 1979$)$, and $S_{23}=([\mathrm{S} \mathrm{II}] \lambda \lambda 6717,31+[\mathrm{S} \mathrm{III}]$ $\lambda \lambda 9069,9532) / \mathrm{H} \beta$ (Díaz and Pérez-Montero, 2000). The methods that consider strongand recombination-lines are more adequate to obtain the chemical abundances of AGNs than the direct method (Dors et al., 2015).

The empirical calibrations are based on the extrapolation of the relations found for the measurements cited above, in order to determine the abundance of objects that do not have prominent emission lines for empirical methods to be applied (see Dors et al., 2011, for more information on abundance estimators). For cases where simplified assumptions and corrections for unseen stages of ionization are not providing reliable estimates of the chemical abundance, one can rely on photoionization models, as the ones from Charlot and Longhetti (2001), Kewley and Dopita (2002), Dors et al. (2011), Nagao et al. (2011), Morisset et al. (2016), and Byler et al. (2018). However, it is important to emphasize that different methods or calibrations for estimating the metallicity show discrepancies of up to $0.3-0.5$ dex in the oxygen abundance estimates (e.g., Stasińska, 2005; Kewley and Ellison, 2008; Dopita et al., 2013; Peimbert et al., 2017). Such differences can be 
explained by the presence of temperature gradients in the high-ionization zones that might not be considered in the methods and calibrations (López-Sánchez et al., 2012), since the $[\mathrm{O} \mathrm{III}] \lambda 4363$ emissivity is biased towards regions of higher temperatures, and that would systematically underestimate the total chemical abundances in all the methods that consider this line explicitly or implicitly by the empirical calibrations (Dopita et al., 2013).

As time progresses, it is expected that the mean metallicity of galaxies will increase as they develop through merging events and accretion processes. The metallicity gradients vary depending on factors as the morphology, the mass and the environment of the galaxy. Gas infall and merger events drive pristine gas from the outer regions of a galaxy into its nucleus, so the central region of an evolved galaxy tends to be a little more metal rich than its outskirts, with a consequent flat gradient profile (see Kewley et al., 2019, and references therein).

With regard to the stellar populations that photoionize the gaseous nebula, the emitted EUV radiation field will be harder in low metallicity environments. That is expected to happen because there are fewer metals in the stellar atmosphere to absorb the radiation field, the massive stars at low metallicity present higher effective temperatures, and the main sequence lifetimes are longer at low metallicity, because of the lower mass-loss rates (Kewley et al., 2019). The effects of WR stars are larger in metal-rich environments, and lower masses are required to reach the WR state (López-Sánchez and Esteban, 2010). In AGNs, the metallicity from the gaseous nebula originates from the past and ongoing star formation near the central SMBH (Collin and Zahn, 1999, Wang et al., 2011).

Most of the elements have only a primary nucleosynthesis, except for nitrogen, carbon, and helium, which have origins that are not solely associated with the thermonuclear processes that happen inside a main-sequence star (Dors et al., 2019). The possible models for the nitrogen abundance will be further detailed in Section 3.3.3. As for the other elements, though many are not explicitly used in the emission-line ratios that we are interested in, it is still important to set their abundances because this will affect the temperature of the nebulae.

For AGNs, we have maintained the default metallicity distribution of CLOUDY, changing only the nitrogen abundance and maintaining the simplest assumptions as possible in the absence of more detailed understanding of the chemical evolution. For H II regions, however, there are several studies with regard to their metallicities, so we defined the abun- 
dances of some elements explicitly. We have adopted the helium and carbon abundances as in Dopita et al. (2006), which are based on empirical fits for $\mathrm{H}$ II regions:

$$
\begin{gathered}
\mathrm{He} / \mathrm{H}=0.0737+0.024 \times\left(Z / Z_{\odot}\right), \\
(\mathrm{C} / \mathrm{H})=6.0 \times 10^{-5} \times\left(Z / Z_{\odot}\right)+2.0 \times 10^{-4} \times\left(Z / Z_{\odot}\right)^{2} .
\end{gathered}
$$

As for the elements that are relevant to dust depletion, we have set an abundance scale of $10 \%$ for iron, magnesium, aluminium, calcium, sodium and nickel, and a scale of $50 \%$ for the silicon abundance (Dors et al., 2011). This is a very crude manner to model the spectra of galactic nuclei seen through the 3" fibre of SDSS, since it does not account for abundance gradients, diffuse emission, or the complex structure of more realistic gaseous nebulae (Stasińska et al., 2006), but can still provide useful guidelines for interpreting observations.

The ranges of metallicity observed for Seyfert galaxies are $0.3 \leq[Z] \leq 2.0$ (Castro et al. 2017), and many of the AGNs in the Local Universe are super-solar (Groves et al. 2004; Dopita et al. 2013; Dors et al., 2020), especially LINERs that are usually found in evolved and massive galaxies (Ho, 2008). The metallicities of star-forming nuclei available for our models are limited to the metallicities at hand for the stellar population synthesis models of Starburst99. Hence, in order to compare the models, we have chosen $[Z]=$ 0.05, 0.2, 0.4, 1.0, 2.0 for star-forming nuclei, and $[Z]=0.4,0.7,1.0,2.0$ for AGNs. In some cases, we have considered an interpolation of the stellar spectra of $[Z]=0.2$ and 0.4 to generate the spectrum for $[Z]=0.3$, a rough estimate valid for lower metallicity regimes.

Dust: It is important to consider dust because of the effects of the depletion of metals into dust grains, that include absorption and scattering of incident radiation, radiation pressure, collisional cooling and photoelectric heating of the gas (Dopita et al., 2002; Groves et al. 2004). The inclusion of dust affects the temperature distribution, since the cooling through infrared line-structure transitions will be less efficient, and that increases the electron temperature, that will cool through more energetic transitions (Spitzer, 1949, Kobulnicky et al., 1999; López-Sánchez et al., 2012; Feltre et al., 2016).

We have considered the presence of dust in the star-forming regions and not in the AGN models, since Castro et al. (2017) shows that models of AGN that assume dust in the gas phase do not reproduce the majority of the expected emission-line intensities 
(Nagao et al., 2006; Dors et al., 2014). The scenario might be different for more complex models of AGNs than considering a constant density, and we will discuss the importance of dust in radiation pressure-dominated models at Appendix A.

We have considered an amount of dust proportional to the metallicity in our models of H II regions, linearly scaling with the oxygen abundance. The abundances of the elements $\mathrm{Mg}, \mathrm{Al}, \mathrm{Ca}, \mathrm{Fe}, \mathrm{Ni}, \mathrm{Na}$, and $\mathrm{Si}$ explained in the above Section were specified in order to account for the depletion of refractary elements onto dust grains (Dors et al., 2011). We have considered the default dust configurations of CLOUDY, which consider a mixture of graphite and silicates with a standard Mathis et al. (1977) grain size distribution and optical properties from Martin and Rouleau (1991) (Feltre et al., 2016).

After explaining each of the parameters in our photoionization models, it is worth pointing out that these codes do not fully represent the complexity of the observed objects. Not all the relevant physical processes are treated correctly, with several simplifications in the temperature, density, ionization, and geometrical structures, and there are considerable uncertainties in the atomic data and stellar atmospheres used Kennicutt et al., 2003; Dors et al., 2011; Kewley et al., 2019). Therefore, the flaws regarding the the synthetic approach to diagnostic diagrams are more related to the simplistic models than to the reliability of the diagrams themselves.

Simplified models are important to better understand how each parameter influences the position of an object in the diagnostic diagrams, with rough quantitative estimates of important parameters. The main parameters that were set in order to build our photoionization models are summarized in Table 3.4 .

In the application of the models to the standard and alternative diagnostic diagrams, we sometimes provide a grid for a range of parameters, and sometimes we provide single points representing specific parameter combinations. This will be further explained below.

\subsubsection{Nitrogen abundance}

Nitrogen is an element with both primary and secondary origins, which makes the determination of its abundance with regard to oxygen more difficult (Vale Asari et al., 2016). Another element that has a secondary origin, carbon, is not used in the standard diagnostic diagrams, thus a detailed description of its abundance is not as crucial as in the 
Table 3.4 - The main parameters and values that are used in the inputs of the photoionization models generated using ClOUdy and Starburst99 in this work.

\begin{tabular}{|c|c|c|}
\hline & Star-forming nuclei & AGN \\
\hline Density $\left(\mathrm{cm}^{-3}\right)$ & 200 & 500 \\
\hline Ionization parameter & $-4.0 \geq \log \mathrm{U} \geq-1.0$ & $-4.0 \geq \log U \geq-1.0$ \\
\hline Geometry & Plane-parallel & Plane-parallel \\
\hline Filling factor & 0.01 & 0.01 \\
\hline SED & $\begin{array}{c}\text { Geneva standard track, } \\
\text { Pauldrach/Hillier atmosphere, } \\
\text { continuous star formation, } \\
10^{6} \text { and } 10^{8} \text { years }\end{array}$ & $\begin{array}{c}\text { BBB at 1.5e5 K } \\
\alpha(\mathrm{UV})=-1.0 \\
\alpha(\mathrm{OX})=-0.8,-1.0,-1.2,-1.4\end{array}$ \\
\hline Metallicity $\left(\mathrm{Z}_{\odot}\right)$ & $0.05,0.2,0.3,0.4,1.0,2.0$ & $0.4,0.7,1.0,2.0$ \\
\hline Dust & Proportional to oxygen abundance & Not included \\
\hline
\end{tabular}

case of nitrogen.

An element that has solely a primary origin will have its production independent of the chemical composition of the stars, that evolve to release it in supernova explosions VilaCostas and Edmunds, 1993), since it is created for the first time in the current generation of stars (Kewley et al., 2019). Hence, the element is independent of the detailed history of the galaxy, and depends on the total amount of matter processed into stars, the IMF, and the nucleosynthetic properties of stars (Lequeux et al., 1979).

The primary nitrogen is produced directly from the fusion of lighter elements, oxygen and carbon that are produced in situ (inside the star and prior to the CNO cycling) due to helium burning in a core, followed by CNO cycling of this material mixed into a hydrogenburning shell. The amount of nitrogen produced may be fairly independent of the initial heavy-element abundance of the star (Alloin et al., 1979, Andrews and Martini, 2013).

The secondary nitrogen is created by the fusion processes that use elements that existed prior to the stars. In this secondary production, the nitrogen is synthesized in the CNO processing of oxygen and carbon in hydrogen burning, and the amount of nitrogen produced is proportional to the initial heavy-element abundance of the star (Kewley et al., 2019).

Both kinds of synthesis can occur in intermediate and massive stars (Renzini and Voli, 1981; Luo et al., 2021). The secondary synthesis should be dominant at higher metallicities $(12+\log (\mathrm{O} / \mathrm{H})>8.0$, Vila-Costas and Edmunds, 1993), while the primary origin dominates for lower metallicity cases, that do not present have the enriched ISM from earlier stellar 
populations to enable the secondary production (Pagel, 2009).

The estimation of the chemical abundance of a certain element (in the example, denominated $X)$ is traditionally done in comparison with metallicity of the $\operatorname{Sun}\left(Z_{\odot}\right)$.

$$
\begin{gathered}
{[Z]=\frac{Z}{Z_{\odot}}} \\
\log \left(\frac{\mathrm{X}}{\mathrm{H}}\right)=\log \left[[\mathrm{Z}]\left(\frac{\mathrm{X}}{\mathrm{H}}\right)_{\odot}\right] .
\end{gathered}
$$

Therefore, it is convenient to provide some of the Solar values that will be considered

$$
\begin{aligned}
& \left(\frac{O}{H}\right)_{\odot}=10^{-3.31}, \\
& \left(\frac{N}{H}\right)_{\odot}=10^{-4.07 .}
\end{aligned}
$$

The value to apply in CLOUDY is

$$
y=\frac{\left(\frac{N}{H}\right)}{[Z]\left(\frac{N}{H}\right)_{\odot}}=\frac{\left(\frac{N}{H}\right)}{[Z] 10^{-4.07}} .
$$

There are several models for the nitrogen abundance in H II regions, and we want to test which is more adequate for describing the observational data.

The comparison between these models and the data could be made directly in the diagram, $\log (\mathrm{N} / \mathrm{O})$ versus $12+\log (\mathrm{O} / \mathrm{H})$, which we will refer to from now on as the $\mathrm{OH}$ NO diagram. However, as mentioned in the last Section 3.3 .2 the chemical abundances determinations often do not agree in their results. Reasons for the scatter in the $\mathrm{OH}$ NO diagram are discussed in Matteucci and Tosi (1985), Henry et al. (2000), Contini et al. (2002), Pilyugin et al. (2003), Pagel (2009), Pérez-Montero and Contini (2009), Dors et al. (2011), among others. The problems with the nitrogen abundance also appear in the diagnostic diagrams, so we will perform a check of the various nitrogen abundance relations proposed which is more adequate to describe our data.

It is also worth mentioning that the objects from our sample are nuclei of galaxies, which are expected to have higher metallicities than the outskirts of the galaxies. Several OH-NO diagrams and relations for the nitrogen abundance are based on $\mathrm{H}$ II regions located in the disks and spiral arms of galaxies, and thus the results could be biased for our particular sample selection (Dopita et al., 2013). 
Since the primary production of nitrogen is independent of the oxygen abundance and the secondary depends on the prior oxygen abundance generated via the CNO cycle, it is expected that the ratio $\mathrm{N} / \mathrm{O}$ will be constant for lower metallicities, where the primary synthesis dominates, and is a function of $\mathrm{O} / \mathrm{H}$ for higher metallicities, where the secondary synthesis becomes relevant (Pérez-Montero and Contini, 2009). However, the transition between these two domains is not clear, and though some authors find an abrupt transition, we will argue for a softer transition, as we have both primary and secondary nitrogen in between the two extreme regimes. Some of the relevant relations for the nitrogen abundance with regard to oxygen in $\mathrm{H}$ II regions are presented next.

The pioneering work of Vila-Costas and Edmunds (1993) considers abundances of nitrogen and oxygen deduced from the spectra of giant H II regions in external galaxies. They find that the $\mathrm{N} / \mathrm{O}$ ratio as a function of $\mathrm{O} / \mathrm{H}$ can be explained by models in which nitrogen has both a (delayed) primary and a secondary component relative to oxygen, with the secondary component dominating at high abundance. The simultaneous presence of both types of nitrogen was not consensus at the time. The proposed Equation is given by

$$
\log \left(\frac{\mathrm{N}}{\mathrm{H}}\right)=\log \left(\frac{\mathrm{O}}{\mathrm{H}}\right)+\log \left[0.034+120\left(\frac{\mathrm{O}}{\mathrm{H}}\right)\right] .
$$

Another relation is proposed by Storchi-Bergmann et al. (1994), where the spectral distributions from the UV to the near-IR of 44 star forming galaxies are considered. Their relation is:

$$
\log \left(\frac{\mathrm{N}}{\mathrm{H}}\right)=\log \left(\frac{\mathrm{O}}{\mathrm{H}}\right)+0.96\left[12+\log \left(\frac{\mathrm{O}}{\mathrm{H}}\right)\right]-9.29 .
$$

Dopita et al. (2000) is a theoretical paper about the recalibration of the extragalactic $\mathrm{H}$ II region sequence. It considers data from $\mathrm{H}$ II regions in 16 galaxies. The proposed nitrogen abundance relation is adapted from an empirical fit from van Zee et al. (1997), whose sample consists of 15 gas-rich, low surface brightness dwarf galaxies.

$$
\log \left(\frac{\mathrm{N}}{\mathrm{H}}\right)= \begin{cases}-4.57+\log [\mathrm{Z}], & \log [\mathrm{Z}] \leq-0.63 \\ -3.94+2 \log [\mathrm{Z}], & \text { otherwise }\end{cases}
$$

However, instead of having two distinct equations, the best relation is probably more continuous, since the primary and secondary processes must coexist at some stages.

Groves et al. (2004) is focused on modeling the NLRs of AGNs. We decided to include 
it in our comparison with the relations for $\mathrm{H}$ II region-like objects

$$
\log \left(\frac{\mathrm{N}}{\mathrm{H}}\right)=\log \left(\frac{\mathrm{O}}{\mathrm{H}}\right)+\log \left[10^{-1.6}+10^{(2.33+\log (\mathrm{O} / \mathrm{H}))}\right] .
$$

Gutkin, Charlot \& Bruzual (2016) model star-forming regions with a large range of metallicities and propose the following relation:

$$
\log \left(\frac{\mathrm{N}}{\mathrm{H}}\right)=\log \left(\frac{\mathrm{O}}{\mathrm{H}}\right)+\log (0.41)+\log \left[10^{-1.6}+10^{(2.33+\log (\mathrm{O} / \mathrm{H}))}\right] .
$$

The last relation for nitrogen abundance from previous works to be explored in this Section is from Nicholls et al. (2017), that propose scaling abundances with total metallicity in nebular photoionization models to replace the uniform scaling normally used in the analysis of $\mathrm{H}$ II regions, finding:

$$
\log \left(\frac{\mathrm{N}}{\mathrm{H}}\right)=\log \left(\frac{\mathrm{O}}{\mathrm{H}}\right)+\log \left[10^{-1.732}+10^{(2.19+\log (\mathrm{O} / \mathrm{H}))}\right]
$$

Besides these relations from the literature, we also attempted our own fit to the data from our sample. Our expression considers a second order term to reach the high values of nitrogen abundance expected for higher abundances. The use of this second order term was proposed by McCall et al. (1985) - see Figure 1.9 in Veilleux and Osterbrock (1987) and Vila-Costas and Edmunds (1993), though the last did not have a large enough sample to perform a fit beyond the first order and linear terms of Equation 3.35. Our best-fit is given by the following relation:

$$
\log \left(\frac{\mathrm{N}}{\mathrm{H}}\right)=\log \left(\frac{\mathrm{O}}{\mathrm{H}}\right)+\log \left[0.039+20\left(\frac{\mathrm{O}}{\mathrm{H}}\right)+1.8 \times 10^{5}\left(\frac{\mathrm{O}}{\mathrm{H}}\right)^{2}\right] .
$$

Figure 3.29 shows how the different relations of nitrogen abundance (dark blue for VilaCostas and Edmunds 1993, blue for Dopita et al. 2000, cyan for Groves et al. 2004, yellow for Gutkin et al.|2016, orange for Nicholls et al. 2017, and red for Equation 3.40) considering a range of metallicities in the BPT diagram. The star-forming sequence mainly depends on the metallicity, so we run only two values for the ionization parameter that cover most of the left branch, which are $\log (U)=-2.0$ in the left panel and $\log (U)=-3.0$ in the right panel. We use models with age of $10^{6}$ years, because these yield harder radiation fields and are more adequate to describe the observational data than older models of $10^{8}$ years, which were tested but are not shown. Here, we show only the BPT diagram and 

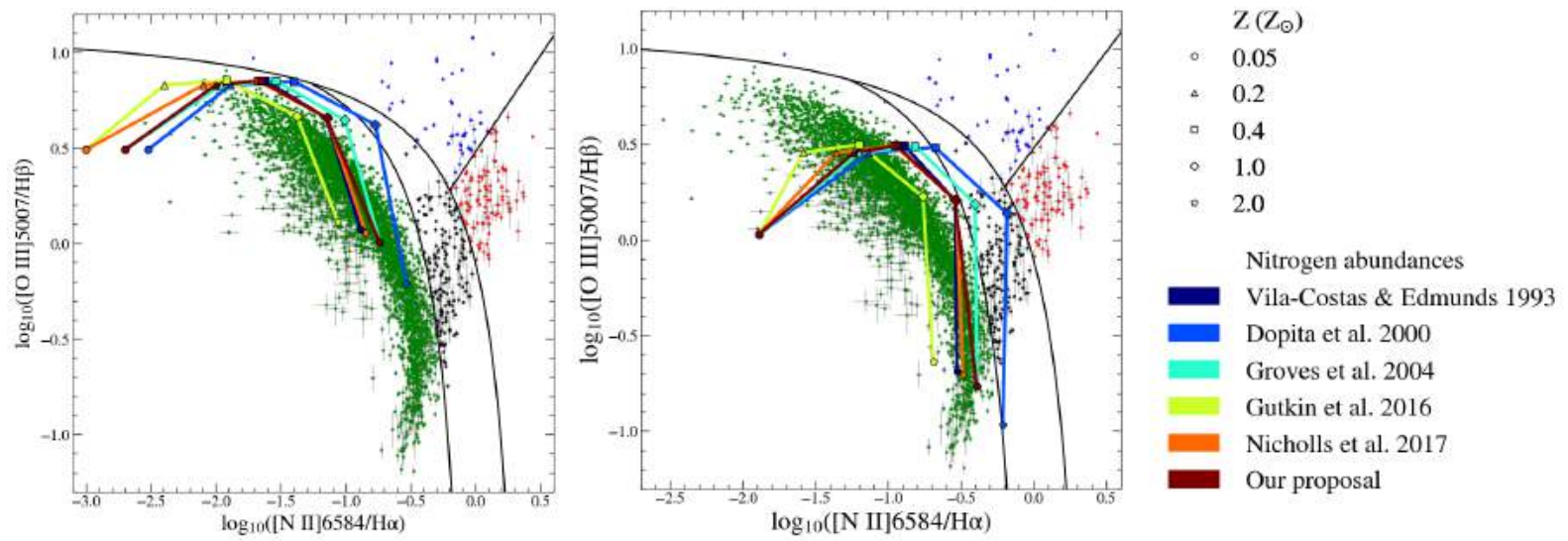

Figure 3.29: BPT diagrams with observational data color-coded according to Kewley et al. (2006) and Cid Fernandes et al. (2010). Points in green are star-forming nuclei, in red are LINERs, in blue are Seyferts, and in black are transition objects. The models of star-forming nuclei are color-coded according to their nitrogen abundance relation and the different markers indicate their metallicities. The left panel indicates models with $\log (U)=-2.0$ and the right panel indicates those with $\log (U)=-3.0$.

not the VO diagrams because we are interested in the behavior of the models with regard to $[\mathrm{N}$ II $]$.

The objects with lower $[\mathrm{O}$ III $] / \mathrm{H} \beta$ require lower values of the ionization parameter, as evident from the panel on the right for $\log (U)=-3.0$. We also notice that the models proposed by Gutkin et al. (2016) (Equation 3.38 represented in yellow) predict the lowest $[\mathrm{N} \mathrm{II}] / \mathrm{H} \alpha$ for the same abundances in comparison to the other models, while Dopita et al. (2000) (Equation 3.36 represented in blue) predicts the highest values for this line ratio. The models that use Equation 3.40 in dark red exhibit similar results than those that consider the nitrogen abundance proposed by Nicholls et al. (2017) in orange(Equation 3.39 .

Figure 3.30 shows two alternative diagnostic diagrams that consider [N II]. These are the same diagrams that were presented in Figures 3.21 (left panel) and 3.25 (right panel). These diagrams are less sensitive to the ionization parameter than the BPT diagram, and considering only the ionization parameter $\log (U)=-3.0$ was sufficient to describe most of the data. Once again, we are showing the models with $10^{6}$ years.

From the left panel of Figure 3.30, we can see that the chemical abundance tracks the star-forming sequence. The line ratio of $[\mathrm{N} \mathrm{II}] / \mathrm{H} \alpha$ is a linear function of the nebular metallicity until the high-metallicity regime, where such ratio saturates around $[\mathrm{N} \mathrm{II}] / \mathrm{H} \alpha \sim$ -0.5 (Kewley and Dopita, 2002; Kewley et al., 2006). That explains the shape of a hook that is observed, and it is one of the reasons why this diagram is so useful to check the 

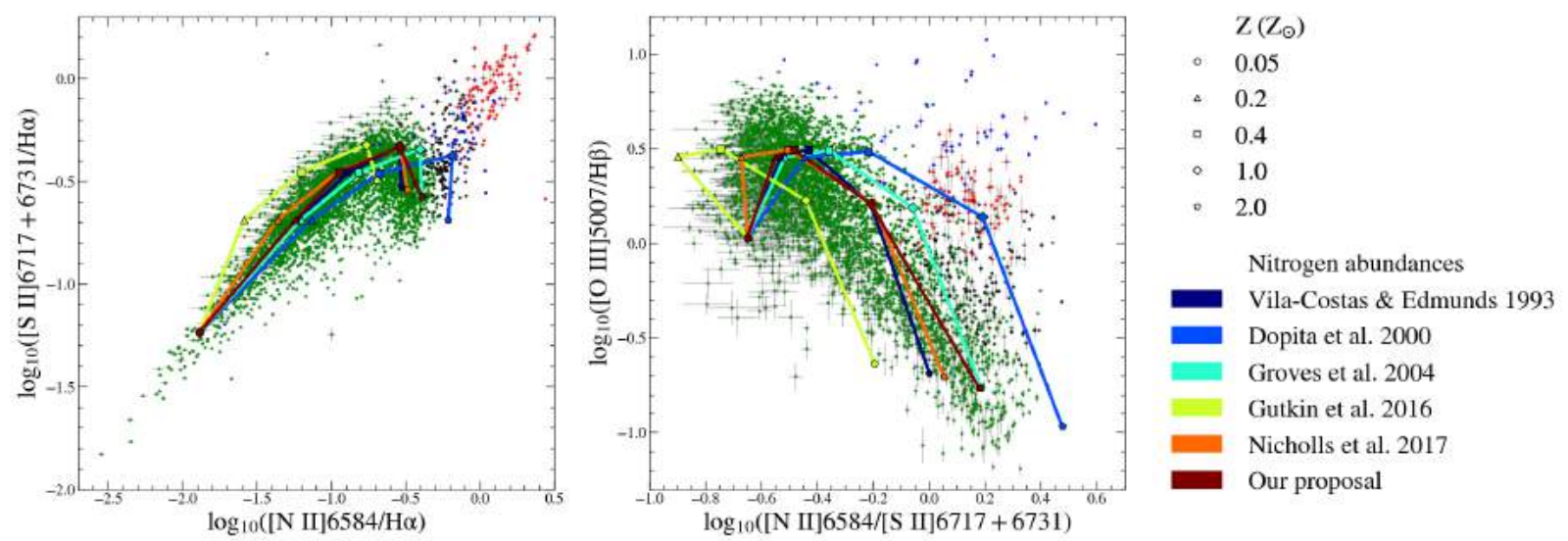

Figure 3.30: Alternative diagnostic diagrams with observational data color-coded according to Kewley et al. (2006) and Cid Fernandes et al. (2010). Points in green are star-forming nuclei, in red are LINERs, in blue are Seyferts, and in black are transition objects. The models of star-forming nuclei are colorcoded according to their nitrogen abundance relation and the different markers indicate their metallicities. All models are considering $\log (U)=-3.0$, since these diagrams are not very sensitive to the ionization parameter.

nitrogen abundance of the models. The panel to the right is useful because the models overlap less and it is easier to differentiate them.

Comparing the results from Figure 3.29 and 3.30 , we see that, once again, the models of the lowest metallicity are coincident in their positions, and the Gutkin et al. (2016) and Dopita et al. (2000) relations represent lower and higher line ratios in comparison with the other relations, respectively. These two models are less representative of the observational data. The main effect of considering different relations for the nitrogen abundance, as expected, is the displacement of the models horizontally, with regard to the intensity of the [N II] emission line. We can see mainly in the left panel of Figure 3.30 that the models that consider the nitrogen abundance proposed in this work (Equation 3.40, in dark red) are the ones that fit better to the observed data from our sample.

Therefore, based on the analysis of this Section, we conclude that the model proposed by Nicholls et al. (2017) (see Equation 3.39 and models color-coded as orange) is the most appropriate among the relations for the nitrogen abundance in $\mathrm{H}$ II regions available in the literature, being also the most similar to the models that consider the nitrogen abundance proposed in this work (Equation 3.40, shown in dark red). However, following the suggestions of McCall et al. (1985) and Vila-Costas and Edmunds (1993), we will continue our analysis with our proposed relation for the nitrogen abundance, considering the second order term (see Equation 3.40 and red lines), since these models are also successful at 
describing the data, as seen in Figures 3.29 and 3.30 .

\subsubsection{Application to diagnostic diagrams}

In this Section, we will apply the theoretical models described in the previous Sections 3.3.1 to 3.3.3 to the standard and alternative diagnostic diagrams, and analyze the reliability of the results.

First, we will present the models that consider Equation 3.40, proposed in this work, for nitrogen abundance relation with regard to oxygen, and the values mentioned in Table 3.4 for the other model parameters. The two main parameters that define the position of starforming nuclei in the diagnostic diagrams are the metallicity and the ionization parameter, so we have run the 5 possible metallicities available for Geneva standard tracks $([Z]=$ $0.05,0.2,0.4,1.0,2.0$, indicated by the circles, triangles, squares, diamonds and pentagons, respectively) and 4 values for the ionization parameter $(U=0.01,0.001,0.0005,0.0001$, indicated by the colors dark blue, light blue, yellow, and dark red, respectively).

We are showing the models with ages of $10^{6}$ years because aging stellar populations emit softer spectra and there is no significant difference in the models with $10^{7}$ and $10^{8}$ years we have tested (D'Agostino et al., 2019).

From Figure 3.31, we see that the proposed photoionization models are successful at describing most of the observational data. The models fold and overlap, in a way that it is not possible to define clearly which are the most adequate metallicity and ionization parameter ranges for each location along the star-forming sequence. This behavior was already expected and it was one of the motivations behind the extreme starburst line proposed by Kewley et al. (2001). However, some of the observed star-forming nuclei with higher values of $[\mathrm{N} \mathrm{II}] / \mathrm{H} \alpha$ from Figure 3.31 are not covered by the models. The main factors that can alter the intensity of $[\mathrm{N} \mathrm{II}] / \mathrm{H} \alpha$ line ratio are a harder ionizing spectrum, a higher density, a higher metallicity, or a combination of these (Yan and Blanton, 2012).

For harder stellar SEDs, some effects associated with the development of stellar atmospheres and tracks that could be included are stellar rotation, binary stellar populations, and WR stars. Other effects as supernovae shocks and diffuse ionized gas could also be included as extra sources that contribute to the ionizing photons in the nuclei of galaxies (Dors et al., 2011; D'Agostino et al., 2019; Kewley et al., 2019). Another relevant issue pointed out by Dopita et al. (2006) is that the STARBURTs99 atmospheres are too soft in 


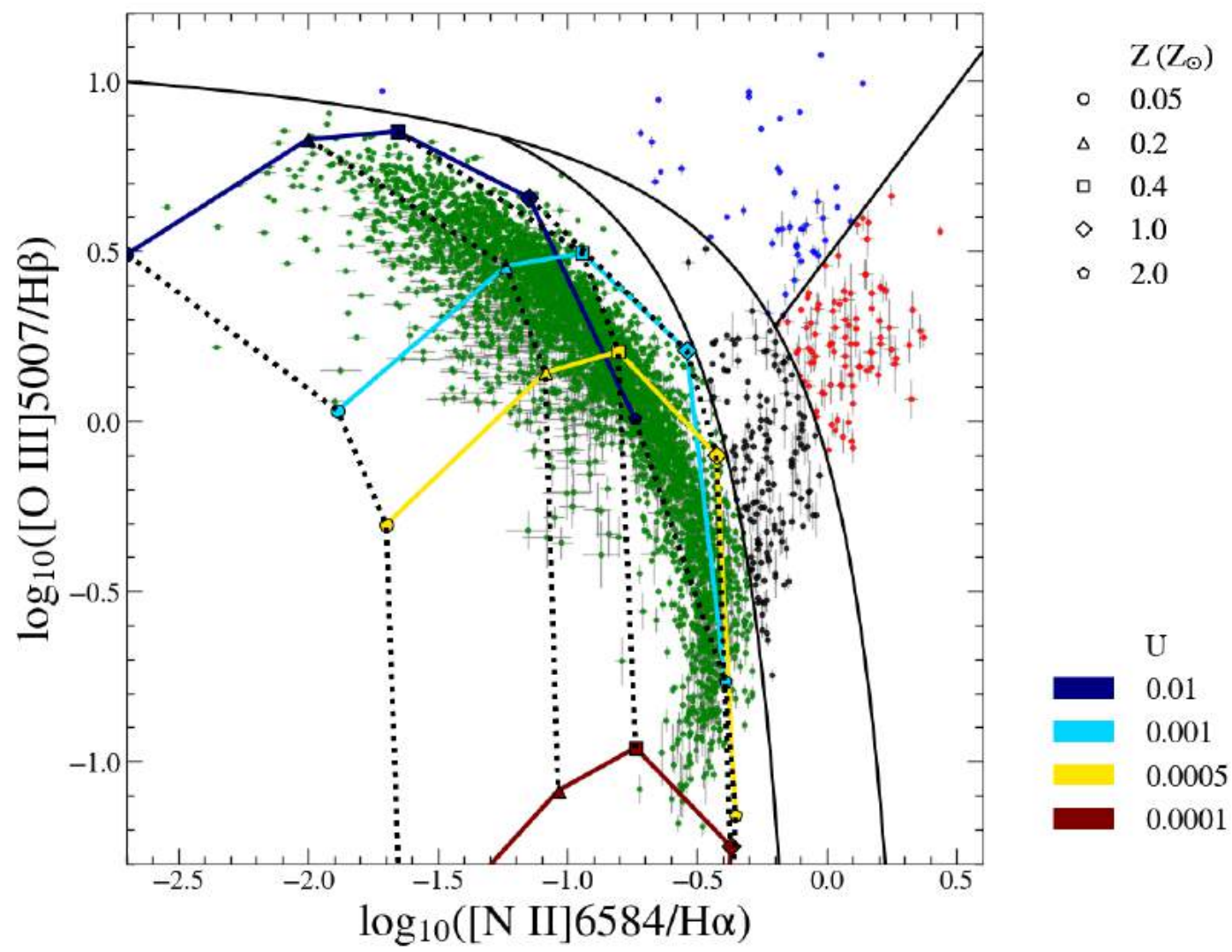

Figure 3.31: BPT diagram with observational data color-coded according to Kewley et al. (2006) and Cid Fernandes et al. (2010). Points in green are star-forming nuclei, in red are LINERs, in blue are Seyferts, and in black are transition objects. The models of star-forming nuclei are color-coded according to the ionization parameter and the different markers indicate their metallicities, as in the label to the right. The dotted lines connect the same metallicity values in order to compose a grid of varying metallicity and ionization parameter.

their EUV spectra, as a consequence of the use of non-clumpy stellar winds.

In Figure 3.32, we have plotted the VO-S diagram for the same models as Figure 3.31. However, this diagnostic diagram is more sensitive to the ionization parameter, and the star-forming nuclei that were in the body of the seagull and not covered by the models in the BPT diagram of Figure 3.31 can now be compared. Some outliers at the low mass and low metallicity end remain, as seen to the left of the star-forming branch.

Figures 3.31 and 3.32 indicate that the main properties that describe the star-forming sequence are the metallicity and the ionization parameter, though the SED could be important, too.

The alternative diagnostic diagram that considers $[\mathrm{N} \mathrm{II}] / \mathrm{H} \alpha$ and $[\mathrm{S} \mathrm{II}] / \mathrm{H} \alpha$ is shown in Figure 3.33. We have decided to not include the classification schemes due to the uncertainty related to the region where the models with the lowest ionization parameter 


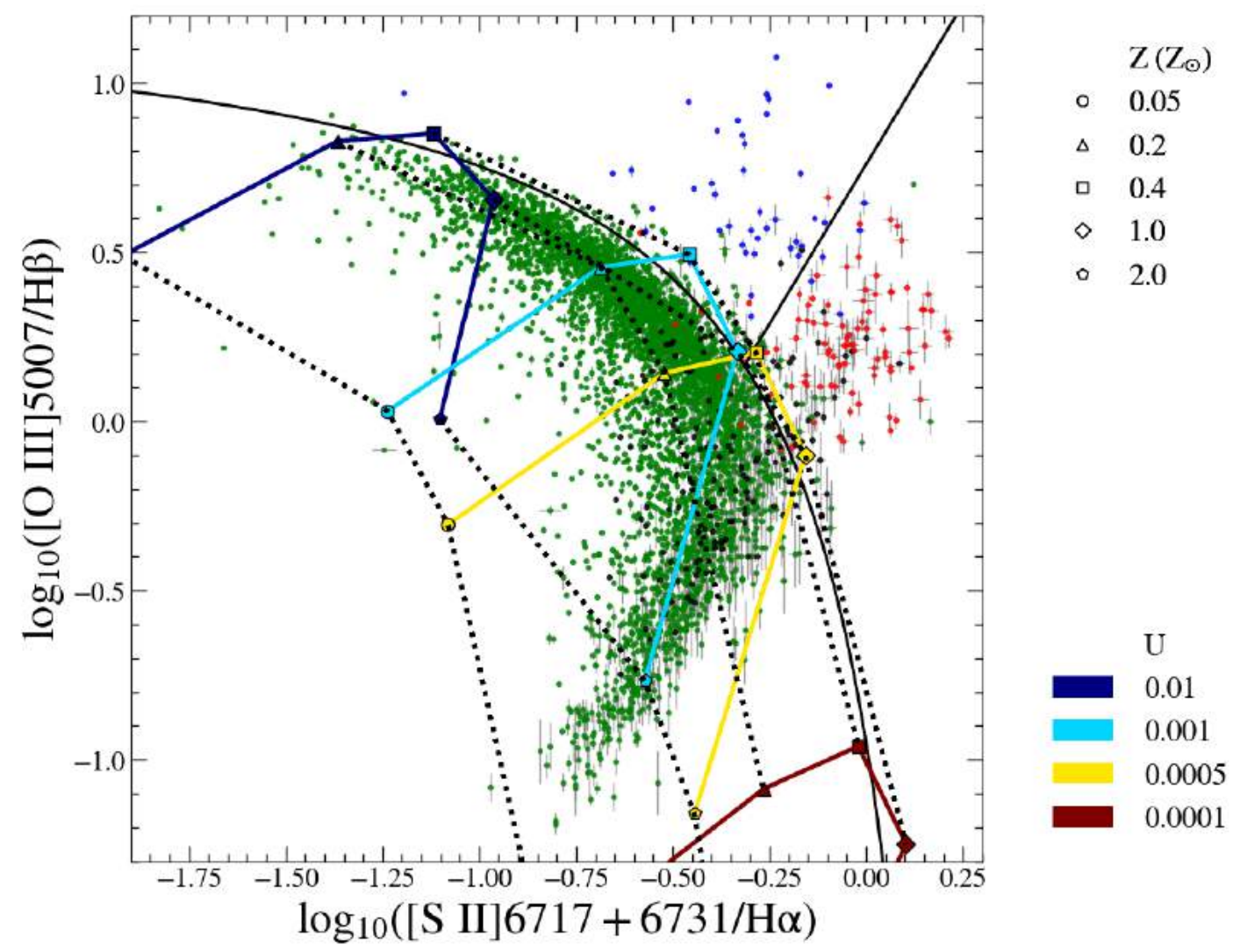

Figure 3.32: VO-S diagram with observational data color-coded according to Kewley et al. 2006) and Cid Fernandes et al. (2010). Points in green are star-forming nuclei, in red are LINERs, in blue are Seyferts, and in black are transition objects. The models of star-forming nuclei are color-coded according to the ionization parameter and the different markers indicate their metallicities, as in the label to the right. The dotted lines connect the same metallicity values in order to compose a grid of varying metallicity and ionization parameter.

(in wine-color) are, since we are not sure if the observational data at this position are H II region-like objects or Seyfert nuclei. As in Figure 3.31, we see that some of the starforming regions with higher values of $[\mathrm{N} \mathrm{II}] / \mathrm{H} \alpha$ are not contemplated by the models, and the probable solutions are the same as stated before (harder ionizing spectrum, higher density, higher metallicity, or a combination of these). We also note the presence of outliers for the cases of lower metallicity, in the tail of the hook-shape that the star-forming sequence delineates in this alternative diagram. However, the models are still adequate to describe the majority of the observed data.

The difficulty in modeling more extreme cases and outliers is more evident on the alternative diagnostic diagram of Figure 3.34. Different values for the density could be tested, but it seems like the main parameters that would solve this issue would be the metallicity and the SED, which are both limited to the models available in STARBURST99. 


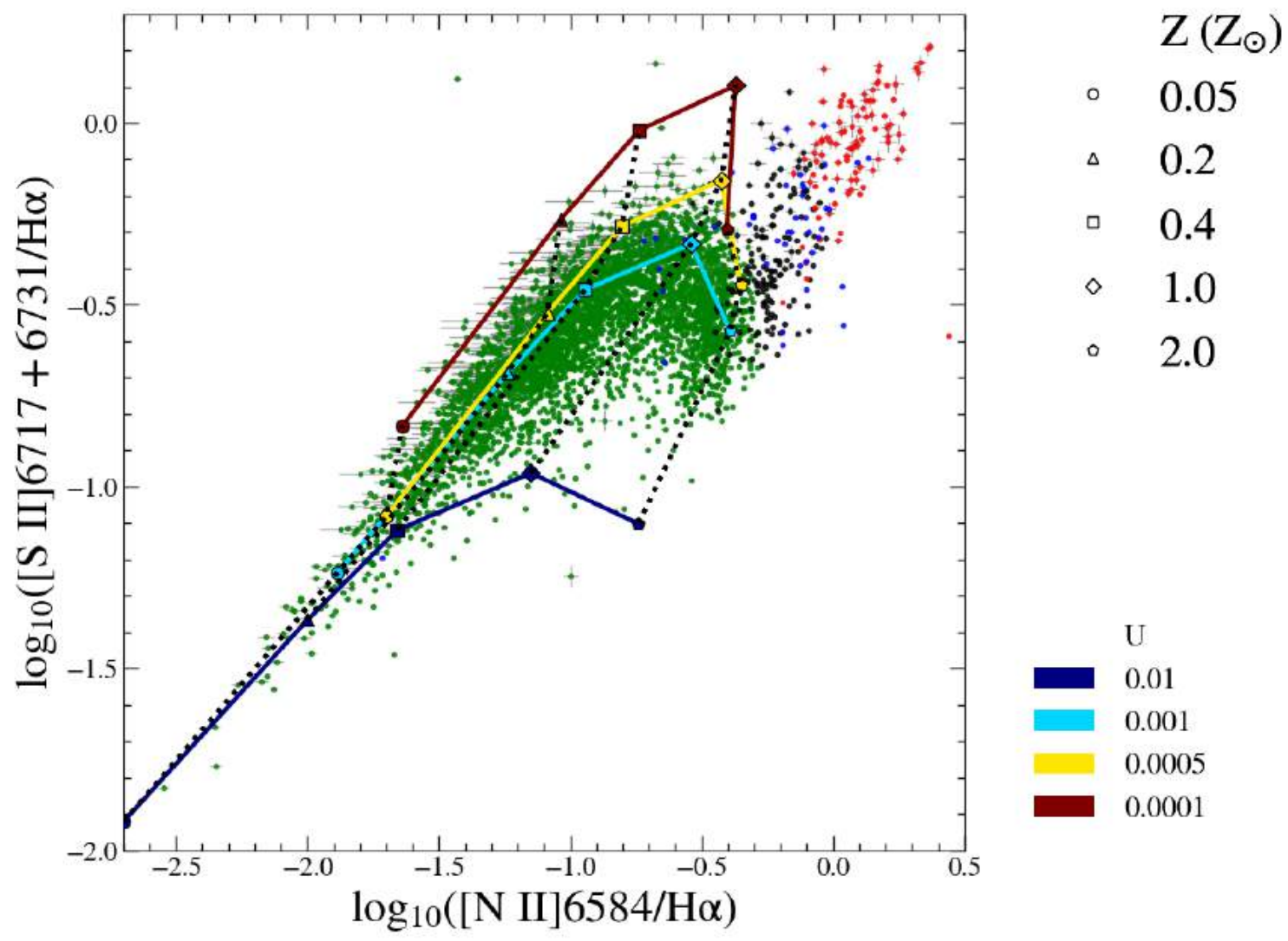

Figure 3.33: Alternative diagnostic diagram from Figure 3.21 with observational data color-coded according to Kewley et al. (2006) and Cid Fernandes et al. (2010). Points in green are star-forming nuclei, in red are LINERs, in blue are Seyferts, and in black are transition objects. The models of star-forming nuclei are color-coded according to the ionization parameter and the different markers indicate their metallicities, as in the label to the right. The dotted lines connect the same metallicity values in order to compose a grid of varying metallicity and ionization parameter.

The next models that will be presented are not in the shape of a grid as the ones that have been shown so far, but they aim to represent an average expectation for the values of the parameters in regions where our objects are found.

Considering fixed metallicities for the stellar SED and the gaseous nebula, we have run several values of the ionization parameter, and chose empirically the ones that represented the largest amount of observational data. These models of H II region-like objects are shown in pink diamonds, with different tones of pink for each set of parameters, as shown in the figure legends.

As for the AGN models, there are three main parameters that define the position of a theoretical model in the diagnostic diagram, which are the ionization parameter, the metallicity, and the spectral index of the power-law SED. We have run several values for the metallicities, and an interesting result, that coincides with the theory that AGN are 


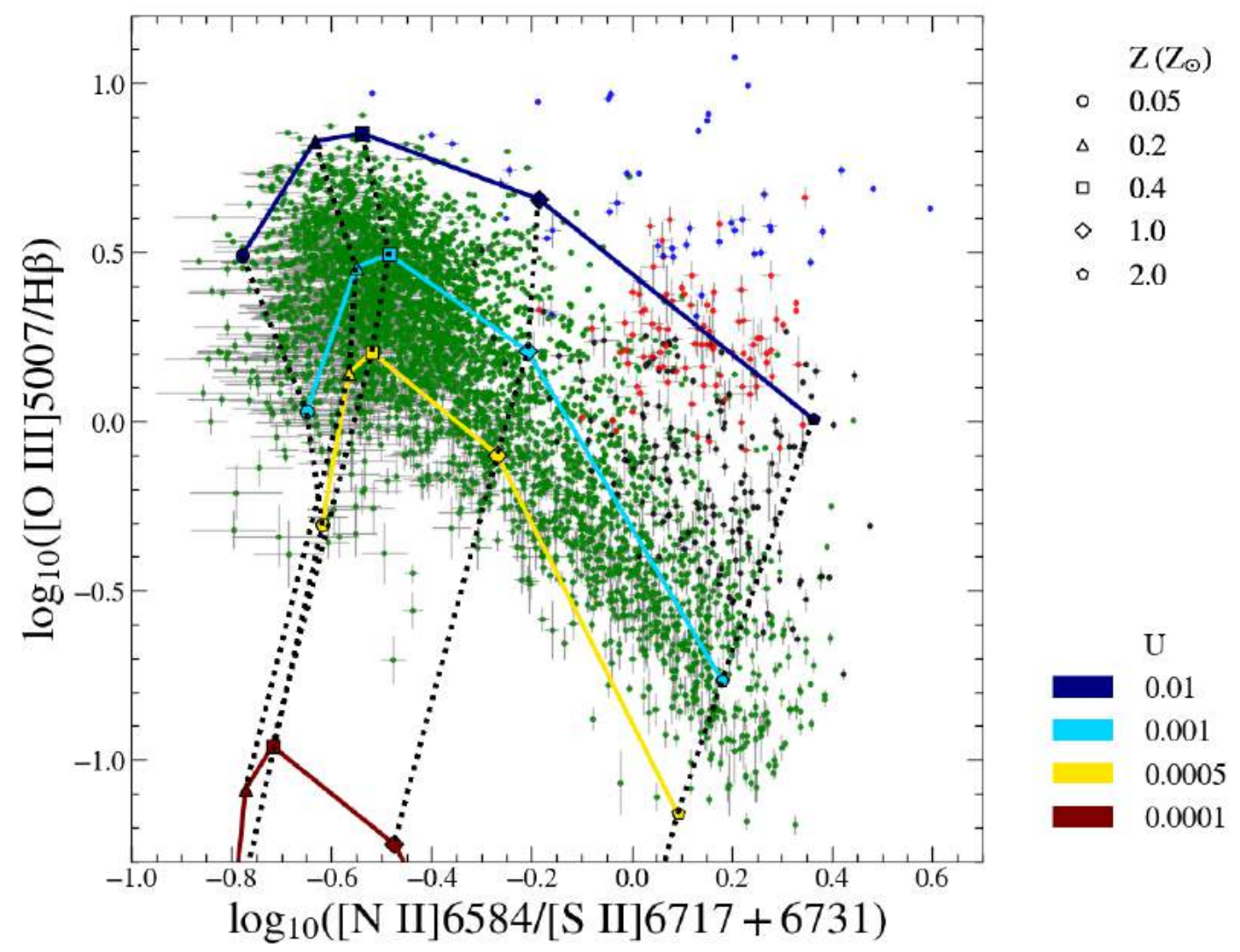

Figure 3.34: Alternative diagnostic diagram from Figure 3.25 with observational data color-coded according to Kewley et al. (2006) and Cid Fernandes et al. (2010). Points in green are star-forming nuclei, in red are LINERs, in blue are Seyferts, and in black are transition objects. The models of star-forming nuclei are color-coded according to the ionization parameter and the different markers indicate their metallicities, as in the label to the right. The dotted lines connect the same metallicity values in order to compose a grid of varying metallicity and ionization parameter.

more common in more massive galaxies, is that the metallicities of once and twice the solar chemical abundance are enough to describe most of the data (Kewley and Dopita, 2002 Groves et al., 2004). Thus, models with $[Z]=1.0$ are represented as triangles and models with $[Z]=2.0$ are represented by squares. The color-code encompasses both ionization parameter and spectral index. Purple models refer to a spectral index of $\alpha=-0.8$, brown models refer to models with $\alpha=-1.2$, and the aquamarine model refers to $\alpha=-1.4$. Once we have found satisfactory models with distinct ionization parameters that have $\alpha=-0.8$ and -1.2 , we have set the darker tones of each color to correspond to lower ionization parameters and the lighter colors for higher values of $\log (U)$. For the diagnostic diagrams shown in Figures 3.35 to 3.40 , the labels are indicated next to the figures.

Figure 3.35 shows the BPT diagnostic diagram for our models of star-forming nuclei and AGNs. With regard to the distinction between Seyferts and LINERs, we see that 


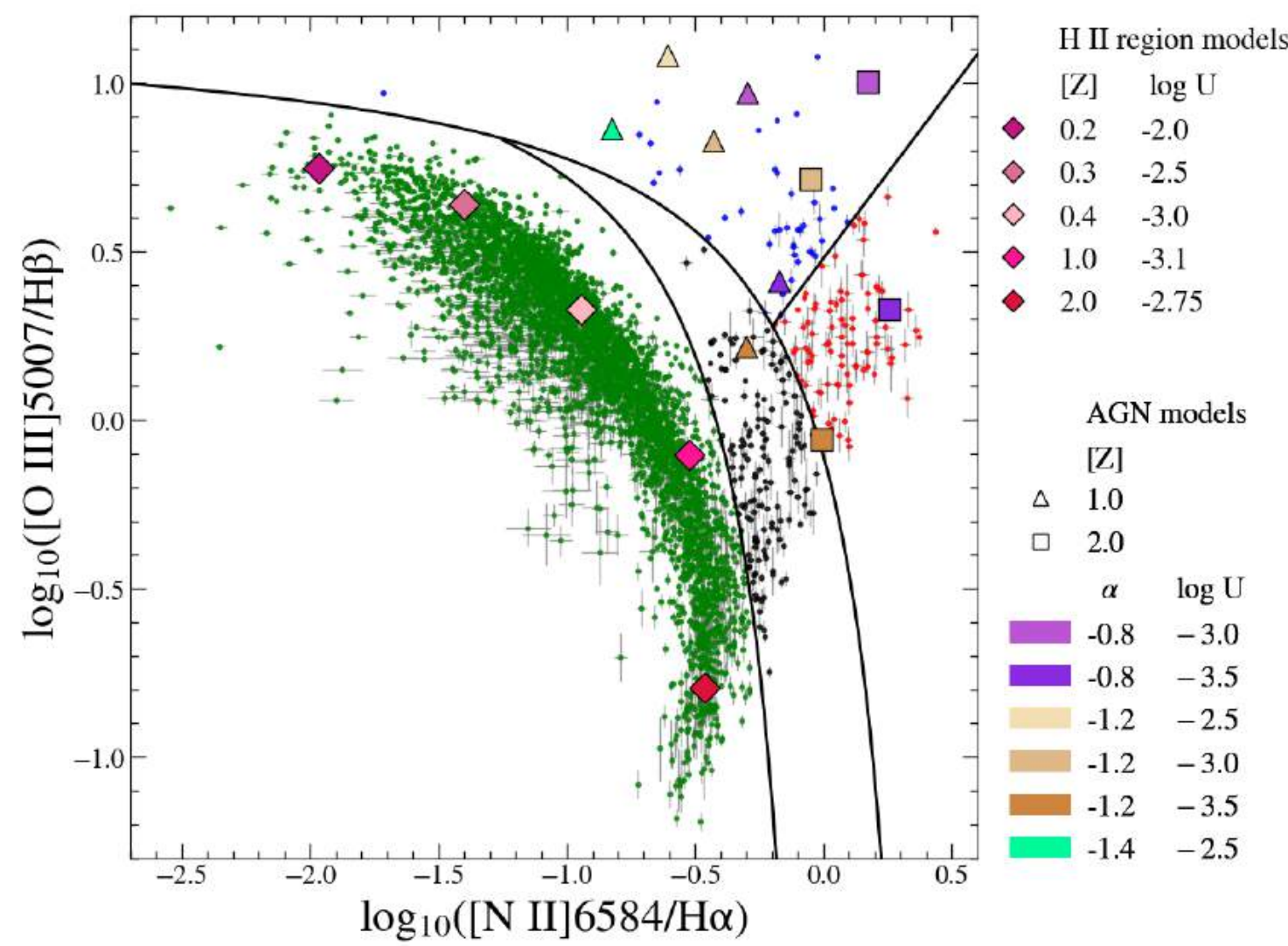

Figure 3.35: BPT diagram with observational data and theoretical models. Points in green are starforming nuclei, in red are LINERs, in blue are Seyferts, and in black are transition objects, according to Kewley et al. (2006) and Cid Fernandes et al. (2010). The models are coded with H II region-like objects (diamonds) having the metallicity and the ionization parameter as variables, and the AGN models (triangles and squares) having the metallicity, the ionization parameter and the power-law spectral index as variables.

the two models that are the most adequate to describe LINERs are the ones with higher metallicities (squares) and lower ionization parameters (darker colors), as expected (Kewley et al., 2006). One of the cases of low ionization parameter and solar metallicity is in the transition region. The other models lie in the Seyfert locus. With regard to the power-law index, models with lower values of $\alpha$ will have lower emission-line ratios for the same fixed parameters (we do not show other cases of $\alpha=-1.4$ because they were in the star-forming branch), while flatter ionizing spectra will have both $[\mathrm{O}$ III $] / \mathrm{H} \beta$ and $[\mathrm{N}$ II $] / \mathrm{H} \alpha$ enhanced (Feltre et al. 2016). The Hit region-like models delineate the star-forming sequence, as expected.

The VO-S diagram of Figure 3.36 also shows a good agreement between the theoretical models and the observational data. However, in the case where there is no transition region, we notice a gap between the models that consider star-formation and the models 


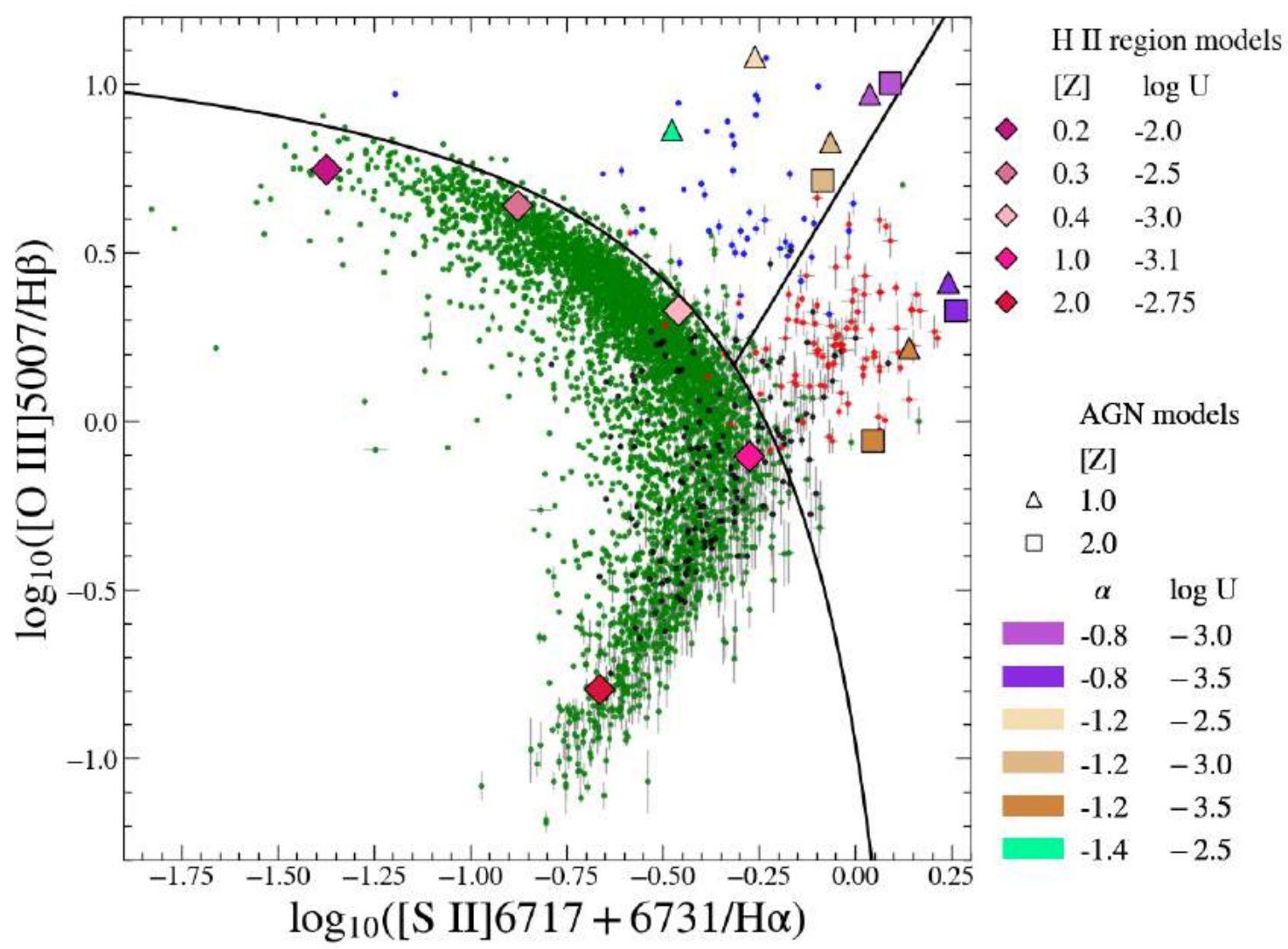

Figure 3.36: VO-S diagram with observational data and theoretical models. Points in green are starforming nuclei, in red are LINERs, in blue are Seyferts, and in black are transition objects, according to Kewley et al. (2006) and Cid Fernandes et al. (2010). The models are coded with H II region-like objects (diamonds) having the metallicity and the ionization parameter as variables, and the AGN models (triangles and squares) having the metallicity, the ionization parameter and the power-law spectral index as variables.

that consider a power-law spectrum. Once again, the star-forming models are adequate to describe the star-forming sequence, which was already shown for our grid in Figure 3.32. As for the AGNs, the separation between high- and low-ionization models among the Seyfert and LINER loci, respectively, is even clearer, since both models with solar and supersolar abundances for the low-ionization cases lie on the LINER locus, with the remaining models on the Seyfert locus.

However, in the VO-O diagram shown in Figure 3.37, the models do not succeed in describing most of the observational data, indicating a trend of lower $[\mathrm{O} \mathrm{I}] / \mathrm{H} \beta$ than expected from our sample. This problem is probably due to the fact that the conditions of the zone that emits the distinct forbidden lines are different. The neutral oxygen emission line is produced mainly in the partially ionized zone, while the once ionized sulfur is produced both in this region and in regions with higher ionization where the once ionized nitrogen 


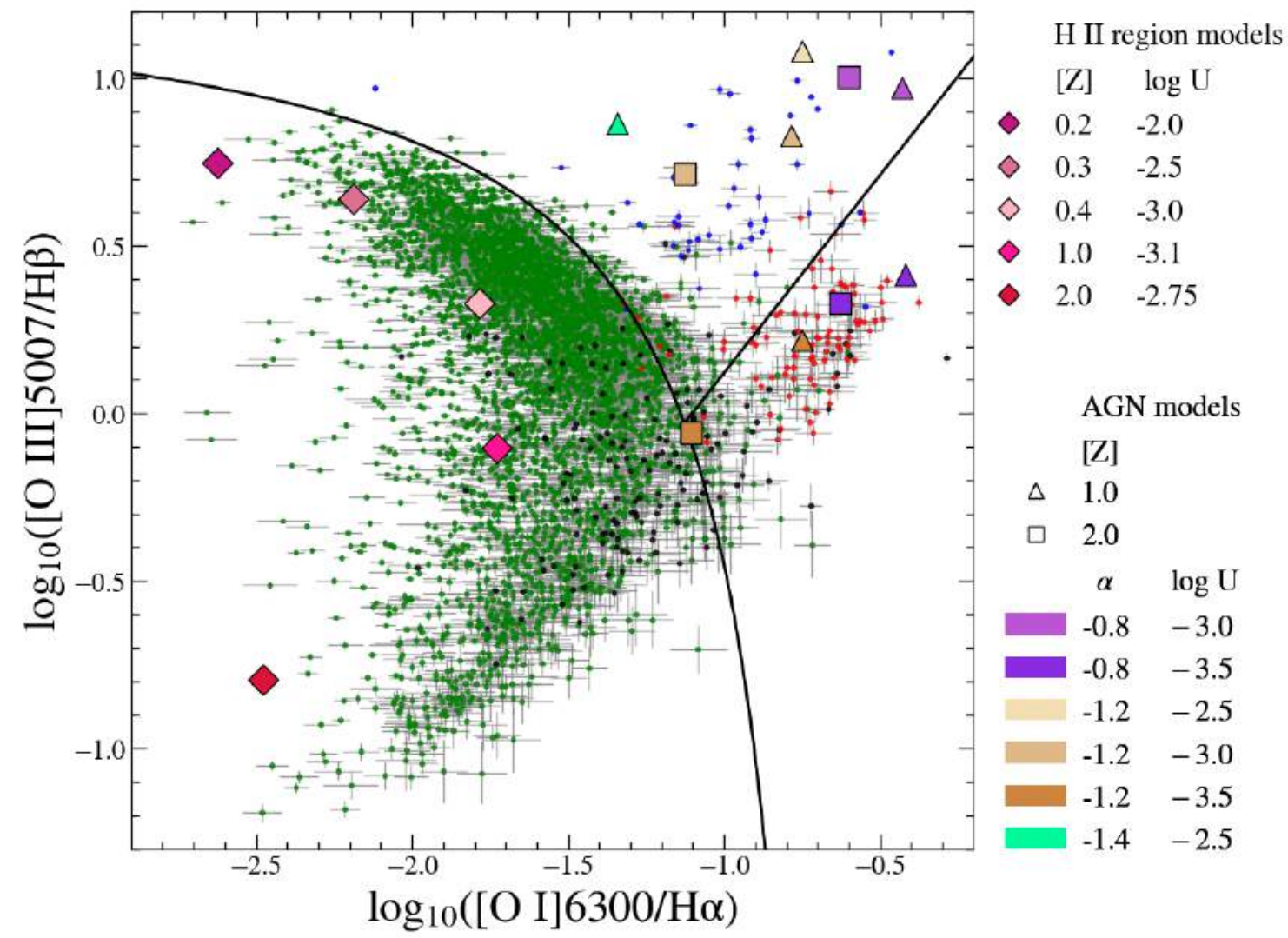

Figure 3.37: VO-O diagram with observational data and theoretical models. Points in green are starforming nuclei, in red are LINERs, in blue are Seyferts, and in black are transition objects, according to Kewley et al. (2006) and Cid Fernandes et al. (2010). The models are coded with H II region-like objects (diamonds) having the metallicity and the ionization parameter as variables, and the AGN models (triangles and squares) having the metallicity, the ionization parameter and the power-law spectral index as variables.

line is produced more efficiently. Hence, the density, ionization parameter and other physical conditions must be different in the regions where these distinct lines are produced. The problems of modeling the distinct emission lines simultaneously with simple models is not new (Stasińska et al., 2006), and a more complex modeling would be necessary to describe the role of each of the different zones that compose a photoionized cloud or region.

As already expected from Figure 3.33, the H II region-like synthetic models are able to reproduce the star-forming sequence in the alternative diagnostic diagram of Figure 3.38. With regard to the AGN models in Figure 3.38, we see more variation with respect to the metallicity, forming two linear trends for each of the fixed metallicities (triangles versus squares), and objects with lower ionization parameters (darker colors) tend to be more to the upper right of the diagram, in agreement with the LINER locus. However, we see that the star-forming and AGN models are closer than in the standard diagnostic 


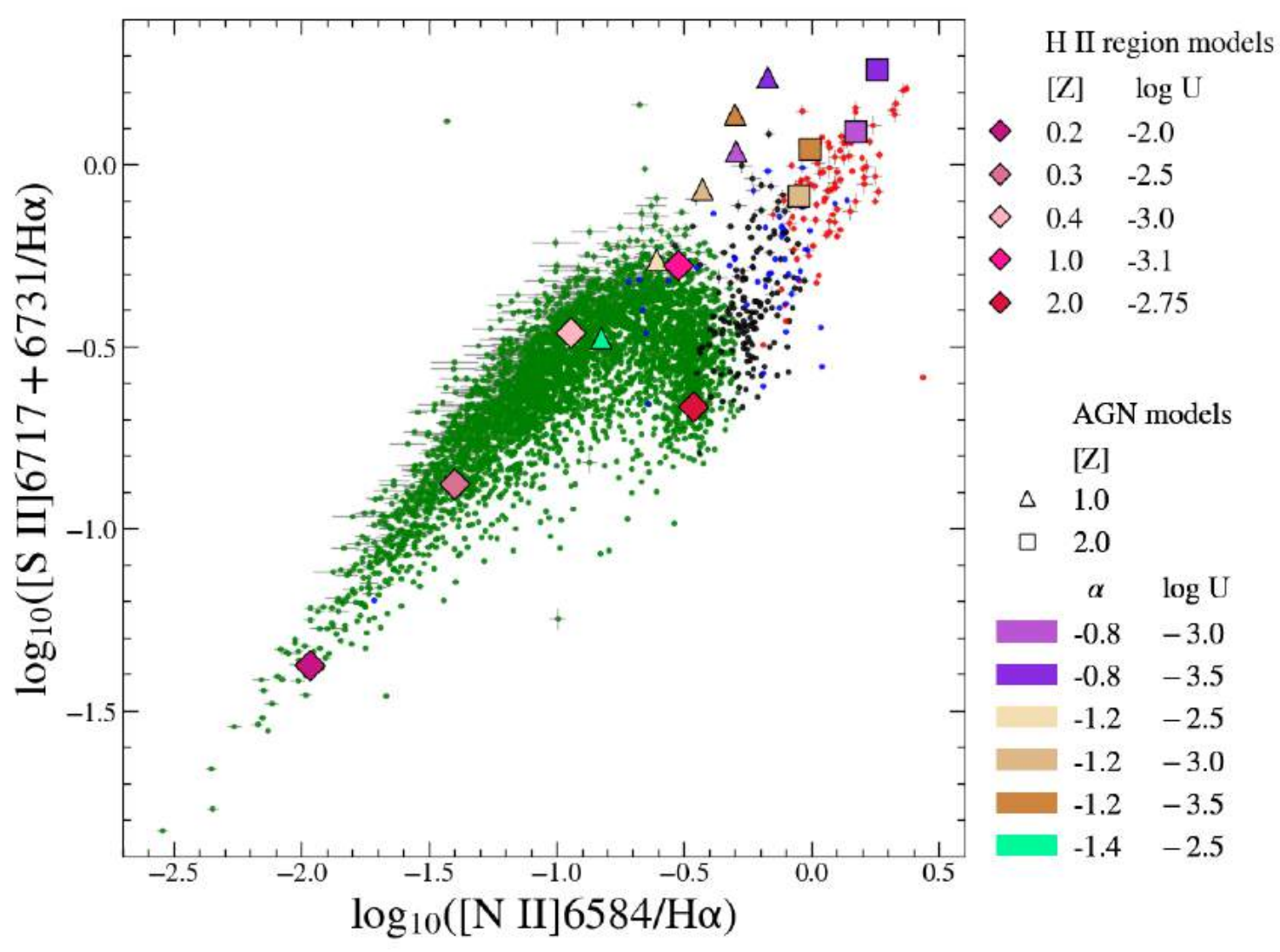

Figure 3.38: Alternative diagnostic diagram from Figure 3.21 with observational data and theoretical models. Points in green are star-forming nuclei, in red are LINERs, in blue are Seyferts, and in black are transition objects, according to Kewley et al. (2006) and Cid Fernandes et al. (2010). The models are coded with $\mathrm{H}$ II region-like objects (diamonds) having the metallicity and the ionization parameter as variables, and the AGN models (triangles and squares) having the metallicity, the ionization parameter and the power-law spectral index as variables.

diagram, which is probably due to the fact that no oxygen lines are being considered. Another relevant issue is that the model with the lowest spectral index (in aquamarine), that showed a low value of $[\mathrm{N} \mathrm{II}] / \mathrm{H} \alpha$ in the BPT diagram of Figure 3.35 is positioned in the star-forming sequence.

The alternative diagnostic diagram of Figure 3.39 shows a reasonable agreement between AGN data and models, but the $\mathrm{H}$ II region-like objects are evidently in disagreement. Such mismatch is due to the fact that the $[\mathrm{OI}] / \mathrm{H} \alpha$ line ratio is being underpredicted in the star-forming nuclei models, as shown in Figure 3.37, and that will have as a result the systematic lowering of the models in comparison to the observational data considering the ordinate axis of this alternative diagram. Therefore, the values that refer to the nitrogen and sulfur emission line ratios are satisfactory, but the values for the oxygen lines are problematic for star-forming objects. One again, we note that the model for $\alpha=-1.4$ lies 


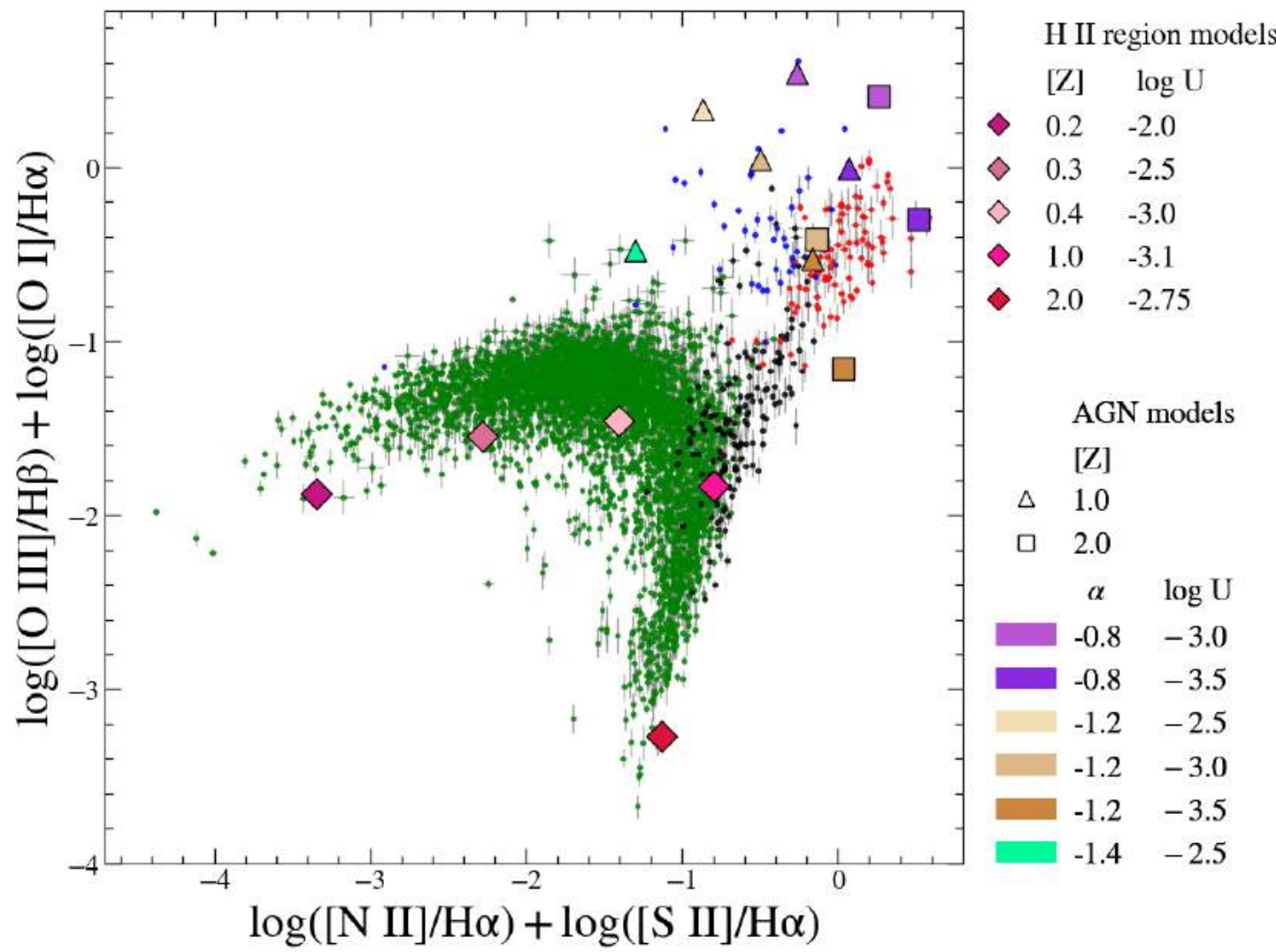

Figure 3.39: Alternative diagnostic diagram from Figure 3.13 with observational data and theoretical models. Points in green are star-forming nuclei, in red are LINERs, in blue are Seyferts, and in black are transition objects, according to Kewley et al. (2006) and Cid Fernandes et al. (2010). The models are coded with $\mathrm{H}$ II region-like objects (diamonds) having the metallicity and the ionization parameter as variables, and the AGN models (triangles and squares) having the metallicity, the ionization parameter and the power-law spectral index as variables.

closer to the star-forming sequence than to the AGN loci. There is a clear trend of higher metallicity AGN gathering in the LINER locus and solar metallicity AGN models being placed at the Seyfert locus. According to the expectation, the models with lower ionization parameter (darker colors) lie in the LINER locus and the remaining models in the Seyfert locus. Moreover, this alternative diagnostic diagram seems appropriate to constrain the spectral index, with the purple flat cases (higher values of $\alpha$ ) being positioned to the upper right and showing a trend with regard to the brown models (lower $\alpha$ ).

Figure 3.40 shows the models applied to the alternative diagnostic diagram of Figure 3.19. It is noticeable that all the models are more to the upper part of the diagram than the distribution of the observational data, again because of the discrepancy in the neutral oxygen emission line from Figure 3.37. The model for $\alpha=-1.4$ is once again closer to the star-forming sequence than the AGN loci. Despite being shifted upwards with regard 


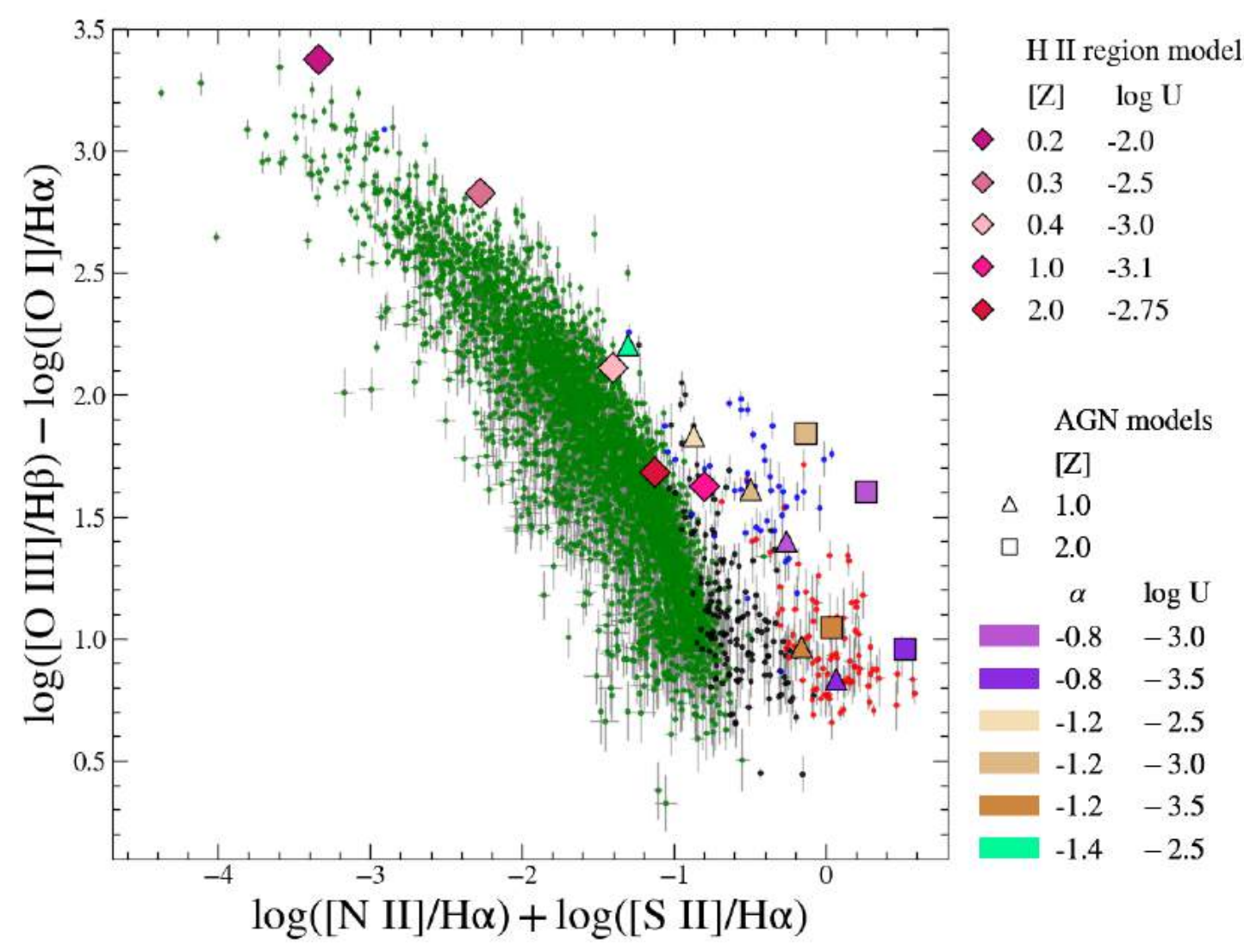

Figure 3.40: Alternative diagnostic diagram from Figure 3.19 with observational data and theoretical models. Points in green are star-forming nuclei, in red are LINERs, in blue are Seyferts, and in black are transition objects, according to Kewley et al. (2006) and Cid Fernandes et al. (2010). The models are coded with $\mathrm{H}$ II region-like objects (diamonds) having the metallicity and the ionization parameter as variables, and the AGN models (triangles and squares) having the metallicity, the ionization parameter and the power-law spectral index as variables.

to the region that is occupied by observational data of AGN, the models are consistent in their own distribution, with objects that have lower ionization parameters being more to the bottom, according to the LINER locus, and the cases of higher metallicity being more to the right in comparison to the models of the same color (same ionization parameter and spectral index), which is expected for the sum of the nitrogen and sulfur line ratios. We also see a trend with regard to the spectral index values, with flatter spectra located more to the lower right, but such behavior is not as explicit as in the case of Figure 3.39 .

Thereby, though the models are adequate to describe most of the considered line ratios $([\mathrm{O} \mathrm{III}] / \mathrm{H} \beta,[\mathrm{N} \mathrm{II}] / \mathrm{H} \alpha$, and $[\mathrm{S} \mathrm{II}] / \mathrm{H} \alpha)$, the fact that they underestimate the ratio of $[\mathrm{O}$ I] $/ \mathrm{H} \alpha$ (see Figure 3.37) makes the models inadequate to apply in the alternative diagnostic diagrams that consider all the standard optical emission line ratios, with systematic deviations in the direction where the neutral oxygen is being considered. 
The issue with modeling the [O I] line, however, is not something exceptional from this work, and has been faced in other works that relied on simplistic photoionization models (e.g., Veilleux and Osterbrock, 1987; Stasińska et al., 2006; Dopita et al., 2013; Dors et al., 2021). Some of the changes that can provide a stronger emission of the neutral oxygen optical emission line is by setting a harder ionization field (by setting higher temperatures, non-equilibrium heating or including shocks), higher densities, or other contributions from dust (Dopita, 1997; Kewley et al., 2006).

LINERs are poorly represented by the models of Figure 3.37. We have tested changing the temperature of the AGN models, by setting the position of the bump to other frequencies than the default, but no significant changes were observed. We have also run models with contributions from shocks and photoionization by a central source provided by professor Dr. Oli Dors in internal collaboration, but such models were still not able to describe the intensity of $[\mathrm{O} \mathrm{I}]$ that the observational data required. The dust, despite of having a role as coolant in several ionization zones, will actually heat the gas in the partially ionized zone. That happens because the grains of dust are being exposed to hard X-ray photons that reach such regions and release higher amounts of energy after photoionizing the gas, heating the medium with the exceeding energy.

By setting models with high densities (as $10^{4}$ and $10^{5} \mathrm{~cm}^{-3}$ ) and intensifying the amount of dust to 2 and 5 times the metallicity scale, we were able to move the AGN models to higher values of $[\mathrm{OI}] / \mathrm{H} \alpha$, as desired. However, though the result on the VO-O diagram is more adequate, the models did not represent LINERs in the BPT and VO-S diagrams well. The cause of the discrepancy is that both [N II] and [S II] lines have critical densities much lower than that of [O I] (Osterbrock and Ferland, 2006), such that the regions where such ions are found are different. In the case where a single high density is assumed, the elements with lower critical densities would have their emission suppressed. It is worth reminding that the emissivity of an emission line is proportional to the square of the density, so there is a trend of preferably detecting the densest regions.

Though the underprediction of the emission line ratio is evident by [O I], this discrepancy of the photoionization models is also seen in other commonly used emission lines such as [Ne III] $\lambda 3869$, and is known as the 'temperature problem' (e.g., Binette et al., 1996) or the '[NeIII] problem' (e.g., Kewley et al., 2019). The missing ionizing photons could also be explained by the contribution of extra sources of ionization rather than solely consid- 
ering the central source, which could be shocks, HOLMES, DIG, turbulent mixing layers, diffuse X-ray emitting plasma, X-ray bursts, cosmic ray heating, and mechanical heating from radio jets in the case of AGNs $(\mathrm{Ho}, 2008)$.

Another important issue to be kept in mind is that each ion observed in a photoionized gaseous nebula is produced in a different region, with different conditions of temperature, density, distance to the power source, etc.. Hence, this makes the interpretation of composite line ratios non-trivial, and different diagnostic diagrams might be tracing distinct regions of the galactic nuclei (Kewley et al., 2019). Therefore, in order to obtain models that satisfy simultaneously all the standard diagnostic diagrams, it is necessary to have more complex models, with a composition of different densities to describe the different regions where each line is preferentially emitted and thus simulate the observed spectra (Stasińska and Schaerer, 1999). A model with a single density is too simplistic to describe the compound photoionized nebulae, and it would be necessary to combine models of clouds with higher and lower densities in order to describe the more extreme cases. Some examples of more complex models of AGNs are presented in Appendix A. 
Chapter 4

\section{Conclusions and Perspectives}

\subsection{Conclusions}

In this work, we provide a review of the use of standard diagnostic diagrams to classify galactic nuclei according to their main ionization and excitation source. We also review some of the less traditional diagnostic diagrams, that have been proposed in the literature, for all the wavelength ranges.

We contextualize the diagrams proposed by Baldwin, Phillips \& Terlevich (1981) (BPT) and by Veilleux and Osterbrock (1987) (VO) based on emission-line ratios $\log ([\mathrm{O} \mathrm{III}] / \mathrm{H} \beta)$, $\log ([\mathrm{N} \mathrm{II}] / \mathrm{H} \alpha), \log ([\mathrm{S} \mathrm{II}] / \mathrm{H} \alpha)$, and $\log ([\mathrm{O} \mathrm{I}] / \mathrm{H} \alpha)$. Then, we analyze some of their classification schemes in our sample of galactic nuclei from the SDSS DR7 Main Galaxy Sample (MPA-JHU data) with $z<0.016$ and with uncertainties of the logarithms of the mentioned emission-line ratios $\epsilon<0.1$ dex. Based on our results and comparing the number of misclassified objects in each classification scheme, we conclude that the lines dividing objects in which the main power source is due to star-formation and due to nuclear activity proposed by Kewley et al. (2006) give the clearest results, though they might be to strict in the VO diagrams. Furthermore, among those object classified AGNs we can successfully

distinguish between Seyferts and LINERs according to the dividing line proposed by Cid Fernandes et al. (2010).

We propose new alternative diagnostic diagrams based on linear combinations of the traditional emission-line ratios on the main axes. We find that these diagrams are also successful at separating the objects according to their main ionization source (see Figures 3.13, 3.15, 3.19, and 3.23, and we propose new classification schemes based on these diagrams (see Equations 3.20 to 3.29 . However, since our alternative diagrams are based 
on the same limited set of spectral features that the standard diagrams also consider, we are not constraining any additional parameters beyond those already constrained by that the BPT and VO diagrams, which means that these diagrams are also not able to discern between the presence of shocks, HOLMES or DIG.

We adopted one of our alternative diagnostic diagrams based on all four emission-line ratios, because it avoids the ambiguity issues that affect the three traditional diagnostic diagrams. However, we note that the objects that are ambiguously classified in the traditional diagrams are often found near the borderlines in the alternative diagnostic diagrams. We conclude that it is important to give proper attention to such non-trivial objects by studying their spectra in more detail, and we emphasize that the equivalent width of $\mathrm{H} \alpha$ and $D_{n}(4000)$ are useful for making a reliable classification of their dominant excitation source.

Though the proposed techniques for separating galactic nuclei according to their dominant power source have been largely successful, it is important to keep in mind that these classification schemes are a rather simplistic approach to understanding the main ionizing mechanisms. Even though this taxonomy helps to broadly characterize galactic nuclei, it is important to note that, in most objects, there will be multiple sources of ionization, and classifying objects only by their main excitation source does not mean that other excitation mechanisms do not contribute to the observed spectra. However, the determination of how much of each ionization mechanism acts on each object is very complex to address directly, and would at least require spectral data at very high spatial resolution.

The main parameters that determine the position of an object in the diagnostic diagrams studied in this thesis are the ionization parameter, the metallicity, and the spectral hardness of the radiation field. Other parameters such as the density, the age in the case of the stellar populations, and the amount of dust are also relevant, but have less effect. Hence, diagrams that are more sensitive to the ionization parameter will clearly distinguish Seyferts (high-ionization AGNs) from LINERs (low-ionization AGNs), and the transition objects will form a bridge between the star-forming nuclei and the LINERs. Star-forming and Seyfert nuclei separate well due to the difference in ionization parameter (see Figures 3.13 and 3.19). In the cases of metallicity-sensitive diagrams, the transition objects form a bridge between star-forming objects and Seyferts because of the mass-metallicity relation. The Seyfert nuclei are more commonly found in the green valley, while LINERs are often 
present in massive early-type galaxies on the red sequence, such that the LINERs represent the most extreme cases comparison to star-forming nuclei from the blue cloud in terms of their metallicity (see Figure 3.23). The alternative diagnostic diagrams proposed are also sensitive to the hardness of the ionization field, as demonstrated with AGN models in which the spectral index was varied (see especially Figure 3.13 , in which the higher spectral indices are toward the top right).

The theoretical photoionization models are very important for constraining how each parameter affects the position of an object in the diagnostic diagrams. However, given the amount of freedom in the models considered, we emphasize that the exact values obtained for each parameter are more relevant for the comparison between the models than as an estimate of the absolute values of these parameters. Though many important insights can be obtained from simplistic models, we show that single-density models are insufficient to describe the complexity of photoionized nebulae, as these objects often have complex structures and show gradients in density, temperature, and ionization parameter. New approaches to deal with this complexity have been proposed in the literature, for example within the context of the composite regions that surround star-forming regions and active supermassive black holes.

One of the main issues that affects the output from the theoretical models is related to the nitrogen abundance. It is well known that the $\mathrm{N} / \mathrm{O}-\mathrm{O} / \mathrm{H}$ relation has a considerable scatter between galaxies. It is challenging to quantify the nitrogen abundance relative to oxygen, due to the complexity of the primary and secondary productions of nitrogen, the presence of both types of production at certain metallicities, and other chemical enrichment processes that occur in the evolution of a galaxy. In this thesis we, therefore, also propose a new expression for the nitrogen abundance in star-forming regions (Equation 3.40), which shows better agreement between the schematic models and the data in the diagrams that are more sensitive to metallicity.

In a summary, in this thesis we have provided an extensive review of the use of diagnostic diagrams to better understand galactic nuclei and their main power source, presented new alternative diagnostic diagrams and new classification schemes based on optical emissionline ratios (or combinations thereof), and a new calibration of the nitrogen abundance used in theoretical models. 


\subsection{Perspectives}

For future work, we intend to extend the expertise obtained in this thesis with regard to diagnostic diagrams of nearby galactic nuclei to those that are at intermediate and high redshift. These objects are known to exhibit higher densities, and AGNs with high accretion rates and/or lower metallicities are much more common. The properties of starforming nuclei at higher redshifts are also different, with the prevalence of more massive stars and binaries, and the expectation of an $\alpha$-enhanced environment, resulting in harder ionizing spectra. Therefore, plotting the data of intermediate- and high-redshift objects in diagnostic diagrams that were originally proposed for local objects can give us insights about the main differences between these galactic nuclei, and will also help us to better understand what are the most significant changes that galactic nuclei undergo as the Universe evolves.

This work will be particularly useful for the interpretation of observations with the Southern Photometric Local Universe Survey (S-PLUS), the James Webb Space Telescope (JWST), and the Subaru Hyper Suprime-Cam (HSC) and Prime Focus Spectrograph (PFS). Also, integral field unity (IFU) spectroscopic data from future telescopes such as the Giant Magellan Telescope (GMT) will guarantee high spacial and spectral resolution from nearby and distant galactic nuclei, so it is important to test our tools to be applied to these data when they are available.

S-PLUS is the largest astronomical survey led by the Brazilian community, and it uses a $0.8 \mathrm{~m}$ telescope in Chile to collect photometric data on a large field of the Southern Hemisphere. This data can be used to identify AGNs up to $z \sim 6$ using photometric techniques.

The Subaru telescope data of interest consist on two surveys: HSC for deep and wide imaging, and PFS for wide-field spectroscopy. Using both types of information, we are able to select and study large samples of intermediate redshift AGNs and obtain medium resolution spectra to construct similar diagnostic diagrams at these redshifts.

Last, the JWST will push the boundaries of knowledge further by its high quality data obtained directly from space, including imaging, spectroscopy, and IFU. With JWST we will be able to apply the standard and alternative rest-frame optical diagnostic diagrams for the first time at $z>4$, where all the important lines have shifted into the near infrared. 
The techniques used in the modeling and interpreting the rest-frame optical spectral diagnostics presented in this thesis will be crucial for analysing and understanding the progenitor objects from the early Universe. 


\section{Bibliography}

Abazajian K. N., Adelman-McCarthy J. K., Agüeros M. A., Allam S. S., et al. The Seventh Data Release of the Sloan Digital Sky Survey, ApJSS, 2009, vol. 182, p. 543

Agostino J. J., Salim S., Crossing the line: Active Galactic Nuclei in the star-forming region of the BPT Diagram, ApJ, 2019, vol. 876, p. 12

Allen M. G., Dopita M. A., Tsvetanov Z. I., Ultraviolet Diagnostics for the Emission-Line Gas in Active Galaxies, ApJ, 1998, vol. 493, p. 571

Allen M. G., Groves B. A., Dopita M. A., Sutherland R. S., Kewley L. J., The MAPPINGS III Library of Fast Radiative Shock Models, ApJS, 2008, vol. 178, p. 20

Allende Prieto C., Lambert D. L., Asplund M., The Forbidden Abundance of Oxygen in the Sun, ApJ, 2001, vol. 556, p. L63

Alloin D., Collin-Souffrin S., Joly M., Vigroux L., Nitrogen and oxygen abundances in galaxies, A\&A, 1979, vol. 78, p. 200

Alonso-Herrero A., Rieke M. J., Rieke G. H., Ruiz M., Using Near-Infrared [Fe II] Lines to Identify Active Galaxies, ApJ, 1997, vol. 482, p. 747

Andrews B. H., Martini P., The Mass-Metallicity Relation with the Direct Method on Stacked Spectra of SDSS Galaxies, ApJ, 2013, vol. 765, p. 140

Antonucci R., Unified models for active galactic nuclei and quasars, ARA\&A, 1993, vol. 31, p. 473 
Armus L., Charmandaris V., Bernard-Salas J., Spoon H. W. W., et al. Observations of Ultraluminous Infrared Galaxies with the Infrared Spectrograph on the Spitzer Space Telescope. II. The IRAS Bright Galaxy Sample, ApJ, 2007, vol. 656, p. 148

Aydar C. P., Steiner J. E., Dispersão de velocidade estelar como parâmetro fundamental na classificação de um núcleo ativo de galáxia, CNPq Report, 2016

Bahcall J. N., Kozlovsky B.-Z., Some Models for the Emission-Line Region of 3c 48, ApJ, 1969a, vol. 158, p. 529

Bahcall J. N., Kozlovsky B.-Z., Some Models of the Emission-Line Region of 3c 273, ApJ, 1969b, vol. 155 , p. 1077

Baldwin J., Ferland G., Korista K., Verner D., Locally Optimally Emitting Clouds and the Origin of Quasar Emission Lines, ApJ, 1995, vol. 455, p. L119

Baldwin J. A., Phillips M. M., Terlevich R., Classification parameters for the emission-line spectra of extragalactic objects, ASP, 1981, vol. 93, p. 5

Balogh M. L., Morris S. L., Yee H. K. C., Carlberg R. G., Ellingson E., Differential Galaxy Evolution in Cluster and Field Galaxies at z 0.3, ApJ, 1999, vol. 527, p. 54

Balzano V. A., Weedman D. W., The near-infrared properties of galactic nuclei, AJ, 1981, vol. 243 , p. 756

Barth A. J., Reichert G. A., Filippenko A. V., Ho L. C., Shields J. C., et al. The Ultraviolet Spectrum of the Liner NGC 4579, ApJ, 1996, vol. 112, p. 1829

Barth A. J., Reichert G. A., Ho L. C., Shields J. C., Filippenko A. V., Puchnarewicz E. M., Ultraviolet Emission from the Liner Nucleus of NGC 6500, ApJ, 1997, vol. 114, p. 2313

Baskin A., Laor A., What controls the [O III $] \lambda 5007$ line strength in active galactic nuclei?, MNRAS, 2005, vol. 358, p. 1043

Beckmann V., Shrader C., Active galactic nuclei. 1st ed. Weinheim: Wiley-VCH Verlag GmbH \& Co., 2012, 374 p.

Belfiore F., Maiolino R., et al. SDSS IV MaNGA - spatially resolved diagnostic diagrams: a proof that many galaxies are LIERs, MNRAS, 2016, vol. 461, p. 3111 
Best P. N., Röttgering H. J. A., Longair M. S., Ionization, shocks and evolution of the emission-line gas of distant 3CR radio galaxies, MNRAS, 2000, vol. 311, p. 23

Bianchi S., Guainazzi M., et al. Evidence for radiation pressure compression in the X-ray narrow-line region of Seyfert galaxies, MNRAS, 2019, vol. 485, p. 416

Binette L., Photoionization models for LINERs: gas distribution abundances, A\&A, 1985, vol. 143 , p. 334

Binette L., González-Gómez D. I., Mayya Y. D., Density Gradients and Internal Dust in the Orion Nebula, RMxAA, 2002, vol. 38, p. 279

Binette L., Magris C. G., Stasińska G., Bruzual A. G., Photoionization in elliptical galaxies by old stars, A\&A, 1994a, vol. 292, p. 13

Binette L., Magris C. G., Stasińska G., Bruzual A. G., Photoionization in elliptical galaxies by old stars, A\&A, 1994b, vol. 292, p. 13

Binette L., Wilson A. S., et al. Photoionization of very high excitation gas in the Circinus galaxy and other active galactic nuclei, A\&A, 1997, vol. 327, p. 909

Binette L., Wilson A. S., Storchi-Bergmann T., Excitation and temperature of extended gas in active galaxies. II. Photoionization models with matter-bounded clouds, A\&A, 1996, vol. 312 , p. 365

Bresolin F., Kennicutt R. C. J., Garnett D. R., The Ionizing Stars of Extragalactic H II Regions, ApJ, 1999, vol. 510, p. 104

Brinchmann J., Charlot S., Heckman T. M., et al. Stellar masses, star formation rates, metallicities and AGN properties for 200,000 galaxies in the SDSS Data Release Two (DR2), Announcement of data release, 2004

Brinchmann J., Charlot S., White S. D. M., Tremonti C., et al. The physical properties of star-forming galaxies in the low-redshift Universe, MNRAS, 2004, vol. 351, p. 1151

Brinchmann J., Pettini M., Charlot S., New insights into the stellar content and physical conditions of star-forming galaxies at $\mathrm{z}=2-3$ from spectral modelling, MNRAS, 2008, vol. 385 , p. 769 
Brown T. M., Bowers C. W., Kimble R. A., et al. Detection and Photometry of Hot Horizontal Branch Stars in the Core of M32, ApJ, 2000, vol. 532, p. 308

Bruzual G., Spectral evolution of galaxies. I. Early-type systems, ApJ, 1983, vol. 273, p. 105

Bruzual G., Charlot S., GALAXEV: Evolutionary Stellar Population Synthesis Models, ASCL, 2011

Burbidge G. R., Burbidge M., Hoyle F., Lynds C. R., On the Interpretation of the Line Spectra of Quasi-Stellar Objects, Natur, 1966, vol. 210, p. 774

Byler N., Dalcanton J. J., Conroy C., Johnson B. D., Nebular Continuum and Line Emission in Stellar Population Synthesis Models, ApJ, 2017, vol. 840, p. 44

Byler N., Dalcanton J. J., Conroy C., Johnson B. D., Choi J., et al. Self-consistent Predictions for LIER-like Emission Lines from Post-AGB Stars, AJ, 2019, vol. 158, p. 2

Byler N., Dalcanton J. J., Conroy C., Johnson B. D., et al. Stellar and Nebular Diagnostics in the Ultraviolet for Star-forming Galaxies, ApJ, 2018, vol. 863, p. 14

Carvalho S. P., Dors O. L., Cardaci M. V., et al. Chemical abundances of Seyfert 2 AGNs - II. Metallicity calibration based on SDSS, MNRAS, 2020, vol. 492, p. 5657

Castro C. S., Dors O. L., Cardaci M. V., Hägele G. F., New metallicity calibration for Seyfert 2 galaxies based on the N2O2 index, MNRAS, 2017, vol. 467, p. 1507

Cerqueira-Campos F. C., Ardila A. R., et al. Coronal-line forest active galactic nuclei - I. Physical properties of the emission-line regions, MNRAS, 2021, vol. 500, p. 2666

Charlot S., Longhetti M., Nebular emission from star-forming galaxies, MNRAS, 2001, vol. 323, p. 887

Chen Y., Bressan A., Girardi L., Marigo P., et al. PARSEC evolutionary tracks of massive stars up to $350 \mathrm{M}_{\odot}$ at metallicities $0.0001 \leq Z \leq 0.04$, MNRAS, 2015, vol. 452, p. 1068

Cid Fernandes R., Heckman T., Schmitt H., González Delgado R. M., Storchi-Bergmann T., Empirical Diagnostics of the Starburst-AGN Connection, ApJ, 2001, vol. 558, p. 81 
Cid Fernandes R., Stasińska G., et al. Alternative diagnostic diagrams and the "forgotten" population of weak line galaxies in the SDSS, MNRAS, 2010, vol. 403, p. 1036

Cid Fernandes R., Stasińska G., Mateus A., Vale Asari N., A comprehensive classification of galaxies in the Sloan Digital Sky Survey: how to tell true from fake AGN?, MNRAS, 2011, vol. 413, p. 1687

Collin S., Zahn J.-P., Star formation and evolution in accretion disks around massive black holes, A\&A, 1999, vol. 344, p. 433

Collins J. A., Rand R. J., Ionization Sources and Physical Conditions in the Diffuse Ionized Gas Halos of Four Edge-On Galaxies, ApJ, 2001, vol. 551, p. 57

Congiu E., Contini M., Ciroi S., Cracco V., et al. High-resolution spectroscopy of the extended narrow-line region of IC 5063 and NGC 7212, MNRAS, 2017, vol. 471, p. 562

Contini M., Line spectra of Liners. The contribution of the different ionization mechanisms, A\&A, 1997, vol. 323, p. 71

Contini M., Shock fronts in the long GRB031203 host galaxy, MNRAS, 2019, vol. 488, p. 4487

Contini M., Viegas-Aldrovandi S. M., Narrow Emission-Line Profiles of Active Galactic Nuclei, ApJ, 1989, vol. 343, p. 78

Contini T., Treyer M. A., Sullivan M., Ellis R. S., Chemical abundances in a UV-selected sample of galaxies, MNRAS, 2002, vol. 330, p. 75

Copetti M. V. F., Mallmann J. A. H., Schmidt A. A., Castañeda H. O., Internal variation of electron density in galactic H II regions, A\&A, 2000, vol. 357, p. 621

Cresci G., Mainieri V., Brusa M., Marconi A., et al. Blowin' in the Wind: Both "Negative" and "Positive" Feedback in an Obscured High-z Quasar, ApJ, 2015, vol. 799, p. 82

da Silva P., Núcleos de galáxias gêmeas morfológicas da Via Láctea: uma amostra completa de 10 objetos, Universidade de São Paulo, 2020, Ph.D. Thesis, 326 p.

da Silva P., Menezes R. B., Steiner J. E., The nuclear region of NGC 613 - I. Multiwavelength analysis, MNRAS, 2020, vol. 492, p. 5121 
D’Agostino J. J., Kewley L. J., Groves B. A., Byler N., et al. Comparison of theoretical starburst photoionization models for optical disgnostics, ApJ, 2019, vol. 878, p. 2

D’Agostino J. J., Kewley L. J., Groves B. A., Medling A., Dopita M. A., Thomas A. D., A new diagnostic to separate line emission from star formation, shocks, and AGNs simultaneously in IFU data, MNRAS, 2019, vol. 485, p. L38

Davidson K., Photoionization and the Emission-Line Spectra of Quasi-Stellar Objects, ApJ, 1972, vol. 171, p. 213

Davies R. L., Dopita M. A., Kewley L., Groves B., et al. The Role of Radiation Pressure in the Narrow Line Regions of Seyfert Host Galaxies, ApJ, 2016, vol. 824, p. 50

de Bruyn A. G., Wilson A. S., The radio properties of Seyfert galaxies, A\&A, 1964, vol. 64, p. 433

de Vaucouleurs G., Color Classification of Galaxies, AJ, 1960, vol. 65, p. 51

de Vaucouleurs G., de Vaucouleurs A., Photographic, Photometric, and Spectroscopic Observations of Seyfert Galaxies, AJ, 1968, vol. 73, p. 858

Díaz A. I., Pérez-Montero E., An empirical calibration of nebular abundances based on the sulphur emission lines, MNRAS, 2000, vol. 312, p. 130

Donahue M., Voit G. M., A Photoionization Model for the Optical Line Emission from Cooling Flows, ApJ, 1991, vol. 381, p. 361

Done C., Gierliński M., Kubota A., Modelling the behaviour of accretion flows in X-ray binaries. Everything you always wanted to know about accretion but were afraid to ask, A\&AR, 2007, vol. 15, p. 1

Dopita M. A., Optical emission from shock waves. I. Abundances in N49, ApJ, 1976, vol. 209, p. 395

Dopita M. A., Optical Emission from Shock Waves. II. Diagnostic Diagrams, ApJS, 1977, vol. 33 , p. 437

Dopita M. A., What Excites FLIERS?, ApJ, 1997, vol. 485, p. L41 
Dopita M. A., Evans I. N., Theoretical Models for H II Regions. II. The Extragalactic H II Region Abundance Sequence, ApJ, 1986, vol. 307, p. 431

Dopita M. A., Fischera J., Sutherland R. S., Kewley L. J., Leitherer C., et al. Modeling the pan-spectral energy distribution of starburst galaxies. III. Emission line diagnostics of ensembles of evolving H II regions, ApJS, 2006, vol. 167, p. 177

Dopita M. A., Groves B. A., Sutherland R. S., et al. Are the Narrow-Line Regions in Active Galaxies Dusty and Radiation Pressure Dominated?, ApJ, 2002, vol. 572, p. 753

Dopita M. A., Kewley L. J., Heisler C. A., Sutherland R. S., A theoretical recalibration of the extragalactic H II region sequence, ApJ, 2000, vol. 542, p. 224

Dopita M. A., Scharwächter J., Shastri P., et al. Probing the physics of narrow-line regions of Seyfert galaxies. I. The case of NGC 5427, A\&A, 2014, vol. 566, p. A41

Dopita M. A., Sutherland R. S., Spectral Signatures of Fast Shocks. II. Optical Diagnostic Diagrams, ApJ, 1995, vol. 455, p. 468

Dopita M. A., Sutherland R. S., Spectral Signatures of Fast Shocks. I. Low-Density Model Grid, ApJS, 1996, vol. 102, p. 161

Dopita M. A., Sutherland R. S., Nicholls D. C., Kewley L. J., Vogt F. P. A., New Strongline Abundance Diagnostics for H II Regions: Effects of $\kappa$-distributed Electron Energies and New Atomic Data, ApJS, 2013, vol. 208, p. 10

Dors O. L., Cardaci M. V., Hägele G. F., et al. On the central abundances of active galactic nuclei and star-forming galaxies, MNRAS, 2015, vol. 453, p. 4102

Dors O. L., Cardaci M. V., Hägele G. F., Krabbe A. C., Metallicity evolution of AGNs from UV emission lines based on a new index, MNRAS, 2014, vol. 443, p. 1291

Dors O. L., Contini et al. Chemical abundances of Seyfert 2 AGNs - IV. Composite models calculated by photoionization + shocks, MNRAS, 2021, vol. 501, p. 1370

Dors O. L., Freitas-Lemes P., et al. Chemical abundances of Seyfert 2 AGNs - I. Comparing oxygen abundances from distinct methods using SDSS, MNRAS, 2020, vol. 492, p. 468 
Dors O. L., Krabbe A., Hägele G. F., Pérez-Montero E., Analysing derived metallicities and ionization parameters from model-based determinations in ionized gaseous nebulae, MNRAS, 2011, vol. 415, p. 3616

Dors O. L., Monteiro A. F., Cardaci M. V., et al. Semi-empirical metallicity calibrations based on ultraviolet emission lines of type-2 AGNs, MNRAS, 2019, vol. 486, p. 5833

Dors O. L., Storchi-Bergmann T., et al. Ages and metallicities of circumnuclear star formation regions from Gemini IFU observations, A\&A, 2008, vol. 482, p. 59

Dors O. L. J., Copetti M. V. F., Abundance gradients in a sample of barred spiral galaxies, A\&A, 2005, vol. 437, p. 837

Elitzur M., Ferland G. J., Radiation Pressure and Emission Clouds around Active Galactic Nuclei, ApJ, 1986, vol. 305, p. 35

Esin A. A., McClintock J. E., Narayan R., Advection-Dominated Accretion and the Spectral States of Black Hole X-Ray Binaries: Application to Nova Muscae 1991, ApJ, 1997, vol. 489 , p. 865

Espinosa-Ponce C., Sánchez S. F., Morisset C., Barrera-Ballesteros J. K., et al. H II regions in the CALIFA survey: I. catalogue presentation, MNRAS, 2020, vol. 494, p. 1622

Evans I. N., Dopita M. A., Theoretical models for H II regions. I. Diagnostic diagrams, AJS, 1985, vol. 58, p. 125

Evans I. N., Dopita M. A., High-Excitation Extranuclear Gas in the Seyfert Galaxy NGC 1068, ApJ, 1986, vol. 310, p. L15

Event Horizon Telescope Collaboration First M87 Event Horizon Telescope Results. VI. The Shadow and Mass of the Central Black Hole, ApJ, 2019, vol. 875, p. L6

Feltre A., Charlot S., Gutkin J., Nuclear activity versus star formation: emission-line diagnostics at ultraviolet and optical wavelengths, MNRAS, 2016, vol. 456, p. 3354

Ferguson J. W., Korista K., Baldwin J., Ferland G., Locally Optimally Emitting Clouds and the Narrow Emission Lines in Seyfert Galaxies, ApJ, 1997, vol. 487, p. 122 
Ferland G., Netzer H., Application of line transfer calculations to active nuclei and novae, ApJ, 1979, vol. 229, p. 274

Ferland G. J., Chatzikos M., Guzmán F., Lykins M. L., van Hoof P. A. M., Williams R. J. R., et al. The 2017 Release CLOUDY, RMxAA, 2017, vol. 53, p. 385

Ferland G. J., Korista K. T., Verner D. A., Ferguson J. W., et al. CLOUDY 90: Numerical Simulation of Plasmas and Their Spectra, PASP, 1998, vol. 110, p. 761

Ferland G. J., Netzer H., Are there any shock-heated galaxies?, ApJ, 1983, vol. 264, p. 105

Ferland G. J., Osterbrock D. E., IUE Spectra and a Resulting Model of Seyfert 2 Galaxies, ApJ, 1986, vol. 300, p. 658

Ferland G. J., Porter R. L., van Hoof P. A. M., Williams R. J. R., Abel N. P., Lykins M. L., et al. The 2013 release of CLOUDY, RMxAA, 2013, vol. 49, p. 137

Fernández-Ontiveros J. A., Spinoglio L., Pereira-Santaella M., Malkan M. A., Andreani P., Dasyra K. M., Far-infrared Line Spectra of Active Galaxies from the Herschel/PACS Spectrometer: The Complete Database, ApJS, 2016, vol. 226, p. 19

Ferrarese L., Merritt D., A Fundamental Relation between Supermassive Black Holes and Their Host Galaxies, ApJ, 2000, vol. 539, p. L9

Filippenko A. V., Halpern J. P., NGC 7213: a key to the nature of LINERs?, ApJ, 1984, vol. 285 , p. 458

Filippenko A. V., Terlevich R., O-Star Photoionization Models of Liners with Weak [O I] lambda 6300 Emission, ApJ, 1992, vol. 397, p. L79

Fioc M., Le Borgne D., Rocca-Volmerange B., PÉGASE: Metallicity-consistent Spectral Evolution Model of Galaxies, ASCL, 2011

Flores-Fajardo N., Morisset C., Stasińska G., Binette L., Ionization of the diffuse gas in galaxies: hot low-mass evolved stars at work, MNRAS, 2011, vol. 415, p. 2182

Flury S. R., Moran E. C., Chemical abundances in active galaxies, MNRAS, 2020, vol. 496, p. 2191 
Franco J., Kurtz S., Hofner P., Testi L., García-Segura G., Martos M., The Density Structure of Highly Compact H II Regions, ApJ, 2000, vol. 542, p. L143

Fukugita M., Ichikawa T., Gunn J. E., Doi M., Shimasaku K., Schneider D. P., The Sloan Digital Sky Survey Photometric System, AJ, 1996, vol. 111, p. 1748

Gabor J. M., Davé R., Finlator K., Oppenheimer B. D., How is star formation quenched in massive galaxies?, MNRAS, 2010, vol. 407, p. 749

García-Benito R., Díaz A., Hägele G. F., et al. Integral field spectroscopy of HII region complexes: the outer disc of NGC6946, MNRAS, 2010, vol. 408, p. 2234

García-Vargas M. L., Mollá M., Martín-Manjón M. L., POPSTAR evolutionary synthesis models - III. Photometric properties of young star clusters and mixed populations, MNRAS, 2013, vol. 432, p. 2746

Garnett D. R., Shields G. A., Skillman E. D., Sagan S. P., Dufour R. J., Interstellar Abundance Gradients in NGC 2403: Comparison to M33, ApJ, 1997, vol. 489, p. 63

Gebhardt K., Bender R., Bower G., Dressler A., Faber S. M., et al. A Relationship between Nuclear Black Hole Mass and Galaxy Velocity Dispersion, ApJ, 2000, vol. 539, p. L13

Genzel R., Eisenhauer F., Gillessen S., The Galactic Center massive black hole and nuclear star cluster, RvMP, 2010, vol. 82, p. 3121

Ghez A. M., Klein B. L., Morris M., Becklin E. E., High Proper-Motion Stars in the Vicinity of Sagittarius A*: Evidence for a Supermassive Black Hole at the Center of Our Galaxy, ApJ, 1998, vol. 509, p. 678

Ghez A. M., Salim S., et al. Measuring Distance and Properties of the Milky Way's Central Supermassive Black Hole with Stellar Orbits, ApJ, 2008, vol. 689, p. 1044

Gillessen S., Eisenhauer F., Trippe S., Alexander T., et al. Monitoring Stellar Orbits Around the Massive Black Hole in the Galactic Center, ApJ, 2009, vol. 692, p. 1075

Goudfrooij P., Hansen L., et al. Interstellar matter in Shapley-Ames elliptical galaxies. II. The distribution of dust and ionized gas, A\&AS, 1994, vol. 105, p. 341 
Goulding A. D., Alexander D. M., Towards a complete census of AGN in nearby Galaxies: a large population of optically unidentified AGN, MNRAS, 2009, vol. 398, p. 1165

Gravity Collaboration Detection of the gravitational redshift in the orbit of the star S2 near the Galactic centre massive black hole, A\&A, 2018, vol. 615, p. L15

Grevesse N., Sauval A. J., Standard Solar Composition, SSR, 1998, vol. 85, p. 161

Groves B. A., Dopita M. A., Sutherland R. S., Dusty, Radiation Pressure-Dominated Photoionization. II. Multiwavelength Emission Line Diagnostics for Narrow-Line Regions, ApJS, 2004, vol. 153, p. 75

Groves B. A., Heckman T. M., Kauffmann G., Emission-line diagnostics of low-metallicity active galactic nuclei, MNRAS, 2006, vol. 371, p. 1559

Gunn J. E., Carr M., Rockosi C., Sekiguchi M., Berry K., Elms B., de Haas E., The Sloan Digital Sky Survey Photometric Camera, AJ, 1998, vol. 116, p. 3040

Gunn J. E., Slegmund W. A., Mannery E. J., Owen R. E., Hull C. L., Leger R. F., The 2.5 m Telescope of the Sloan Digital Sky Survey, AJ, 2006, vol. 131, p. 2332

Gutiérrez L., Beckman J. E., The Galaxy-wide Distributions of Mean Electron Density in the H II Regions of M51 and NGC 4449, ApJ, 2010, vol. 710, p. L44

Gutkin J., Charlot S., Bruzual G., Modelling the nebular emission from primeval to present-day star-forming galaxies, MNRAS, 2016, vol. 462, p. 1757

Halpern J. P., Grindlay J. E., X-ray photoionized nebulae, ApJ, 1980, vol. 242, p. 1041

Halpern J. P., Steiner J. E., Low ionization active galactic nuclei : X-ray or shock heated?, ApJ, 1983, vol. 269, p. L37

Hao L., Wu Y., et al. Probing the Excitation of Extreme Starbursts: High-Resolution Mid-Infrared Spectroscopy of Blue Compact Dwarfs, ApJ, 2009, vol. 704, p. 1159

Hatchett S., Buff J., McCray R., Transfer of X-rays through a spherically symmetric gas cloud, ApJ, 1976, vol. 206, p. 847 
Heckman T., Borthakur S., Wild V., Schiminovich D., Bordoloi R., COS-burst: Observations of the Impact of Starburst-driven Winds on the Properties of the Circum-galactic Medium, ApJ, 2017, vol. 846, p. 151

Heckman T. M., An optical and radio survey of the nuclei of bright galaxies. Activity in normal galactic nuclei., A\&A, 1980, vol. 87, p. 152

Heckman T. M., Starburst galaxies from Encyclopedia of Astronomy \& Astrophysics of P. Murdin, IOPP, 2006

Heckman T. M., Best P. N., The coevolution of galaxies and supermassive black holes: Insights from surveys of the contemporary Universe, ARA\&A, 2014, vol. 52, p. 589

Henry R. B. C., Edmunds M. G., Köppen J., On the Cosmic Origins of Carbon and Nitrogen, ApJ, 2000, vol. 541, p. 660

Herrera-Camus R., Bolatto A., et al. The Ionized Gas in Nearby Galaxies as Traced by the [N II] 122 and $205 \mu \mathrm{m}$ Transitions, ApJ, 2016, vol. 826, p. 175

Hillier D. J., Miller D. L., The Treatment of Non-LTE Line Blanketing in Spherically Expanding Outflows, ApJ, 1998, vol. 496, p. 407

Ho I.-T., Kewley L. J., Dopita M. A., Medling A. M., et al. The SAMI Galaxy Survey: shocks and outflows in a normal star-forming galaxy, MNRAS, 2014, vol. 444, p. 3894

Ho L. C., Optical Spectroscopy of LINERs and Low-Luminosity Seyfert Nuclei, ASPC, 1996, vol. 103, p. 103

Ho L. C., Nuclear activity in nearby galaxies, ARA\&A, 2008, vol. 46, p. 475

Ho L. C., Filippenko A. V., Sargent W. L. W., A Reevaluation of the Excitation Mechanism of LINERs, ApJ, 1993, vol. 417, p. 63

Ho L. C., Filippenko A. V., Sargent W. L. W., A search for "dwarf" Seyfert nuclei. III. Spectroscopic parameters and properties of the host galaxies, ApJSS, 1997, vol. 112, p. 315

Hogg D. W., Finkbeiner D. P., Schlegel D. J., Gunn J. E., A Photometricity and Extinction Monitor at the Apache Point Observatory, AJ, 2001, vol. 122, p. 2129 
Hsieh B. C., Lin L., Lin J. H., Pan H. A., et al. SDSS-IV MaNGA: Spatially Resolved Star Formation Main Sequence and LI(N)ER Sequence, ApJL, 2017, vol. 851, p. L24

Hubble E. P., Realm of the Nebulae. New Haven: Yale University Press, 1936, 288 p.

Inskip K. J., Best P. N., Rawlings S., Longair M. S., Cotter G., Röttgering H. J. A., Eales S., Deep spectroscopy of $\mathrm{z} \sim 16 \mathrm{C}$ radio galaxies - I. The effects of radio power and size on the properties of the emission-line gas, MNRAS, 2002, vol. 337, p. 1381

Jensen E. B., Strom K. M., Strom S. E., Composition Gradients in Spiral Galaxies: a Consistency Check on the - Wave Theory, ApJ, 1976, vol. 209, p. 748

Kakkad D., Groves B., Dopita M., Thomas A. D., et al. Spatially resolved electron density in the narrow line region of $\mathrm{z}<0.02$ radio AGNs, A\&A, 2018, vol. 618, p. A6

Kallman T., McCray R., The Bowen Resonance Fluorescence Mechanism in Cosmic X-ray sources, BAAS, 1982, vol. 11, p. 720

Kashino D., Silverman J. D., Sanders D., Kartaltepe J. S., Daddi E., Renzini A., Valentino F., et al. The FMOS-COSMOS Survey of Star-forming Galaxies at z $\approx 1.6$. IV. Excitation State and Chemical Enrichment of the Interstellar Medium, ApJ, 2017, vol. 835, p. 88

Kauffmann G., Heckman T. M., Feast and Famine: regulation of black hole growth in low-redshift galaxies, MNRAS, 2009, vol. 397, p. 135

Kauffmann G., Heckman T. M., Tremonti C., Brinchmann J., Charlot S., et al. The host galaxies of active galactic nuclei, MNRAS, 2003, vol. 336, p. 1055

Keel W. C., Spectroscopic evidence for activity in the nuclei of normal spiral galaxies, ApJ, 1983, vol. 269, p. 466

Kennicutt R. C. J., Bresolin F., Garnett D. R., The Composition Gradient in M101 Revisited. II. Electron Temperatures and Implications for the Nebular Abundance Scale, ApJ, 2003, vol. 591, p. 801

Kennicutt R. C. J., Garnett D. R., The Composition Gradient in M101 Revisited. I. H II Region Spectra and Excitation Properties, ApJ, 1996, vol. 456, p. 504 
Kewley L. J., Dopita M. A., Using strong lines to estimate abundances in extragalactic H II regions and starburst galaxies, ApJS, 2002, vol. 142, p. 35

Kewley L. J., Dopita M. A., Sutherland R. S., Heisler C. A., J. T., The host galaxies of active galactic nuclei, ApJ, 2001, vol. 556, p. 221

Kewley L. J., Ellison S. L., Metallicity Calibrations and the Mass-Metallicity Relation for Star-forming Galaxies, ApJ, 2008, vol. 681, p. 1183

Kewley L. J., Groves B., Heckman T. M., The host galaxies and classification of active galactic nuclei, MNRAS, 2006, vol. 372, p. 961

Kewley L. J., Maier C., Yabe K., Ohta K., Akiyama M., Dopita M. A., Yuan T., The cosmic BPT diagram: confronting theory with observations, ApJ, 2013, vol. 774, p. L10

Kewley L. J., Nicholls D. C., Sutherland R. S., Understanding Galaxy Evolution Through Emission Lines, ARA\&A, 2019, vol. 57, p. 511

Kobulnicky H. A., Kennicutt R. C. J., Pizagno J. L., On Measuring Nebular Chemical Abundances in Distant Galaxies Using Global Emission-Line Spectra, ApJ, 1999, vol. 514, p. 544

Kobulnicky H. A., Phillips A. C., Measuring Global Galaxy Metallicities Using EmissionLine Equivalent Widths, ApJ, 2003, vol. 599, p. 1031

Komossa S., Schulz H., Interpretation of the emission line spectra of Seyfert 2 galaxies by multi-component photoionization models, A\&A, 1997, vol. 323, p. 31

Kormendy J., Ho L. C., Coevolution (Or Not) of Supermassive Black Holes and Host Galaxies, ARA\&A, 2013, vol. 51, p. 511

Koski A. T., Spectrophotometry of Seyfert 2 galaxies and narrow-line radio galaxies, ApJ, 1978, vol. 223, p. 56

Krolik J. H., McKee C. F., Hydrogen emission-line spectra in quasars and active galactic nuclei, ApJS, 1978, vol. 37, p. 459

Kroupa P., The Initial Mass Function of Stars: Evidence for Uniformity in Variable Systems, Sci, 2002, vol. 295, p. 82 
Krumholz M. R., Fumagalli M., da Silva R. L., Rendahl T., Parra J., SLUG - stochastically lighting up galaxies - III. A suite of tools for simulated photometry, spectroscopy, and Bayesian inference with stochastic stellar populations, MNRAS, 2015, vol. 452, p. 1447

Lamareille F., Mouhcine M., Contini T., et al. The luminosity-metallicity relation in the local Universe from the 2dF Galaxy Redshift Survey, MNRAS, 2004, vol. 350, p. 396

Lamers H., Maeder A., Schmutz W., Cassinelli J. P., Wolf-Rayet Stars as Starting Points or as Endpoints of the Evolution of Massive Stars?, ApJ, 1991, vol. 368, p. 538

Laor A., On the Nature of Low-Luminosity Narrow-Line Active Galactic Nuclei, ApJ, 2003, vol. 590, p. 86

Laor A., Draine B. T., Spectroscopic Constraints on the Properties of Dust in Active Galactic Nuclei, ApJ, 1993, vol. 402, p. 441

Laurent O., Mirabel I. F., Charmandaris V., Gallais P., et al. Mid-infrared diagnostics to distinguish AGNs from starbursts, A\&A, 2000, vol. 359, p. 887

Leitherer C., Heckman T. M., Synthetic Properties of Starburst Galaxies, ApJS, 1995, vol. 96 , p. 9

Leitherer C., Schaerer D., Goldader J. D., Delgado R. M. G., et al. Starburst99: synthesis models for galaxies with active star formation, ApJS, 1999, vol. 123, p. 3

Lequeux J., Peimbert M., Rayo J. F., et al. Chemical composition and evolution of irregular and blue compact galaxies, A\&A, 1979, vol. 80, p. 155

Levesque E. M., Kewley L. J., Larson K. L., Theoretical Modeling of Star-Forming Galaxies. I. Emission-Line Diagnostic Grids for Local and Low-Metallicity Galaxies, AJ, 2010, vol. 139 , p. 712

Loaiza-Agudelo M., Overzier R. A., Heckman T. M., VLT/X-Shooter Spectroscopy of Lyman Break Analogs: Direct-method O/H Abundances and Nitrogen Enhancements, ApJ, 2020, vol. 891, p. 19

López-Cobá C., Sánchez S. F., et al. Systematic study of outflows in the Local Universe using CALIFA: I. Sample selection and main properties, MNRAS, 2019, vol. 482, p. 4032 
López-Sánchez A. R., Dopita M. A., Kewley L. J., et al. Eliminating error in the chemical abundance scale for extragalactic H II regions, MNRAS, 2012, vol. 426, p. 2630

López-Sánchez a. R., Esteban C., Massive star formation in Wolf-Rayet galaxies. IVb. Using empirical calibrations to compute the oxygen abundance, arXiv, 2010

Luo Y., Heckman T., Hwang H.-C., Rowlands K., Sánchez-Menguiano L., Riffel R., et al. Evidence for the Accretion of Gas in Star-forming Galaxies: High N/O Abundances in Regions of Anomalously Low Metallicity, ApJ, 2021, vol. 908, p. 183

Luridiana V., Cerviño M., The Effect of Density Structure and Aperture on Nebular Diagnostics, ASPC, 2003, vol. 297, p. 49

Lynden-Bell D., Galactic Nuclei as Collapsed Old Quasars, Natur, 1969, vol. 223, p. 690

MacAlpine G. M., On QSO and Seyfert galaxy line-emission models, ApJ, 1974, vol. 193, p. 37

Magorrian J., Tremaine S., Richstone D., Bender R., Bower G., Dressler A., et al. The Demography of Massive Dark Objects in Galaxy Centers, ApJ, 1998, vol. 115, p. 2285

Maier C., Lilly S. J., Carollo C. M., et al. Oxygen Gas Abundances at z 1.4: Implications for the Chemical Evolution History of Galaxies, ApJ, 2006, vol. 639, p. 858

Maoz D., Koratkar A., Shields J. C., Ho L. C., Filippenko A. V., Sternberg A., The Ultraviolet Spectra of LINERs: A Comparative Study, ApJ, 1998, vol. 116, p. 55

Martin P. G., Rouleau F., Extreme Ultraviolet Opacity with Interstellar Dust, EUA, 1991, p. 341

Mateus A., Sodré L., et al. Semi-empirical analysis of Sloan Digital Sky Survey galaxies II. The bimodality of the galaxy population revisited, MNRAS, 2006, vol. 370, p. 721

Mathez G., On the Forbidden Line Spectra of QSS, A\&A, 1968, vol. 3, p. 127

Mathis J. S., Rumpl W., Nordsieck K. H., The size distribution of interstellar grains, ApJ, 1977, vol. 217 , p. 425

Matsuoka K., Nagao T., Marconi A., Maiolino R., Mannucci F., et al. The mass-metallicity relation of high-z type-2 active galactic nuclei, A\&A, 2018, vol. 616, p. L4 
Matteucci F., Tosi M., Nitrogen and oxygen evolution in dwarf irregular galaxies, MNRAS, 1985, vol. 217 , p. 391

McCall M. L., Rybski P. M., Shields G. A., The chemistry of galaxies. I. The nature of giant extragalactic H II regions, AJS, 1985, vol. 57, p. 1

McLeod A. F., Gritschneder M., Dale J. E., Ginsburg A., Klaassen P. D., Mottram J. C., et al. Connecting the dots: a correlation between ionizing radiation and cloud mass-loss rate traced by optical integral field spectroscopy, MNRAS, 2016, vol. 462, p. 3537

Meléndez M., Heckman T. M., Martínez-Paredes M., Kraemer S. B., Mendoza C., Theoretical modelling of emission-line galaxies: new classification parameters for mid-infrared and optical spectroscopy, MNRAS, 2014, vol. 443, p. 1358

Meléndez M., Kraemer S. B., et al. Uncovering the Spectral Energy Distribution in Active Galaxies Using High-ionization Mid-infrared Emission Lines, ApJ, 2011, vol. 738, p. 6

Menezes R. B., Steiner J. E., Ricci T. V., da Silva P., Nicolazzi D. M., First results of the DIVING $^{3 D}$ survey of bright galaxies in the local universe: The mini-DIVING ${ }^{3 D}$ sample, IAUS, 2020, vol. 359, p. 450

Miller B. P., Brandt W. N., Schneider D. P., et al. X-ray Emission from Optically Selected Radio-intermediate and Radio-loud Quasars, ApJ, 2011, vol. 726, p. 20

Miniutti G., Ponti G., Greene J. E., Ho L. C., et al. The XMM -Newton view of AGN with intermediate-mass black holes, MNRAS, 2009, vol. 394, p. 443

Monreal-Ibero A., Arribas S., Colina L., LINER-like Extended Nebulae in ULIRGs: Shocks Generated by Merger-Induced Flows, ApJ, 2006, vol. 637, p. 138

Moran E. C., Filippenko A. V., Chornock R., "Hidden" Seyfert 2 Galaxies and the X-Ray Background, ApJ, 2002, vol. 579, p. L71

Morgan W. W., Mayall N. U., A Spectral Classification of Galaxies, PASP, 1957, vol. 69, p. 291

Morisset C., Delgado-Inglada G., Sánchez S. F., Galbany L., et al. Photoionization models of the CALIFA H II regions. I. Hybrid models, A\&A, 2016, vol. 594, p. 37 
Moustakas J., Kennicutt R. C. J., Tremonti C. A., et al. Optical Spectroscopy and Nebular Oxygen Abundances of the Spitzer/SINGS Galaxies, ApJS, 2010, vol. 190, p. 233

Moy E., Rocca-Volmerange B., The balance between shocks and AGN photoionization in radio sources and its relation to the radio size, A\&A, 2002, vol. 383, p. 46

Nagao T., Maiolino R., Marconi A., Gas metallicity diagnostics in star-forming galaxies, A\&A, 2006, vol. 459, p. 85

Nagao T., Maiolino R., Marconi A., Matsuhara H., Metallicity diagnostics with infrared fine-structure lines, A\&A, 2011, vol. 526, p. 149

Netzer H., Radiation Pressure Force and Black Hole Mass Determination in Low-Redshift Type-I and Type-II Active Galactic Nuclei, ApJ, 2009, vol. 695, p. 793

Netzer H., The physics and evolution of active galactic nuclei. 1st ed. New York: Cambridge University Press, 2013, 353 p.

Netzer H., Revisiting the Unified Model of Active Galactic Nuclei, ARA\&A, 2015, vol. 53, p. 365

Nicholls D. C., Sutherland R. S., Dopita M. A., Kewley L. J., Groves B. A., Abundance scaling in stars, nebulae and galaxies, MNRAS, 2017, vol. 466, p. 4403

Noeske K. G., Weiner B. J., Faber S. M., Papovich C., et al. Star Formation in AEGIS Field Galaxies since $\mathrm{z}=1.1$ : The Dominance of Gradually Declining Star Formation, and the Main Sequence of Star-forming Galaxies, ApJ, 2007, vol. 660, p. L43

O'Donnell J. E., R v-dependent Optical and Near-Ultraviolet Extinction, AJ, 1994, vol. 422 , p. 158

Osterbrock D. E., Active galactic nuclei, QJRAS, 1984, vol. 25, p. 1

Osterbrock D. E., Ferland G. J., Astrophysics of gaseous nebulae and active galactic nuclei. 2nd ed. Sausalito: University Science Books, 2006, 461 p.

Osterbrock D. E., Parker R. A. R., Excitation of the Optical Emission Lines in QuasiStellar Radio Sources, ApJ, 1966, vol. 143, p. 268 
Overzier R. A., Heckman T. M., Tremonti C., Armus L., et al. Local Lyman Break Galaxy Analogs: The Impact of Massive Star-Forming Clumps on the Interstellar Medium and the Global Structure of Young, Forming Galaxies, ApJ, 2009, vol. 706, p. 203

Pagel B. E. J., Nucleosynthesis and chemical evolution of galaxies. 2nd ed. New York: Cambridge University Press, 2009, 466 p.

Pagel B. E. J., Edmunds M. G., Blackwell D. E., et al. On the composition of H II regions in southern galaxies - I. NGC 300 and 1365, MNRAS, 1979, vol. 189, p. 95

Pauldrach A. W. A., Hoffmann T. L., Lennon M., Radiation-driven winds of hot luminous stars. XIII. A description of NLTE line blocking and blanketing towards realistic models for expanding atmospheres, A\&A, 2001, vol. 375, p. 161

Peimbert M., Peimbert A., Delgado-Inglada G., Nebular Spectroscopy: A Guide on Hii Regions and Planetary Nebulae, PASP, 2017, vol. 129, p. 2001

Pérez E., González Delgado R., Vílchez J. M., Density structure of the giant HII region NGC 2363, ApSSS, 2001, vol. 277, p. 83

Pérez-Montero E., Ionized Gaseous Nebulae Abundance Determination from the Direct Method, PASP, 2017, vol. 129, p. 3001

Pérez-Montero E., Contini T., The impact of the nitrogen-to-oxygen ratio on ionized nebula diagnostics based on [NII] emission lines, MNRAS, 2009, vol. 398, p. 949

Pérez-Montero E., Dors O. L., Vílchez J. M., García-Benito R., Cardaci M. V., Hägele G. F., A bayesian-like approach to derive chemical abundances in type-2 active galactic nuclei based on photoionization models, MNRAS, 2019, vol. 489, p. 2652

Phillips M. M., Charles P. A., Baldwin J. A., Nearby galaxies with Seyfert-like nuclei, AJ, 1983, vol. 266, p. 485

Phillips M. M., Jenkins C. R., Dopita M. A., et al. Ionized gas in elliptical and SO galaxies. I. A survey for H alpha and N II emission, ApJ, 1986, vol. 91, p. 1062

Pilyugin L. S., Thuan T. X., Vílchez J. M., On the origin of nitrogen, A\&A, 2003, vol. 397, p. 487 
Poetrodjojo H., Groves B., Kewley L. J., et al. The SAMI Galaxy Survey: Spatially resolved metallicity and ionization mapping, MNRAS, 2018, vol. 479, p. 5235

Ramos-Larios G., Phillips J. P., Pérez-Grana J. A., The nature of the compact HII region Sh 2-89 and its stellar content, MNRAS, 2010, vol. 405, p. 245

Raymond J. C., Shock waves in the interstellar medium, ApJS, 1979, vol. 39, p. 1

Renzini A., Voli M., Advanced evolutionary stages of intermediate-mass stars, A\&A, 1981, vol. 94, p. 175

Revalski M., Crenshaw D. M., Kraemer S. B., Fischer T. C., et al. Quantifying Feedback from Narrow Line Region Outflows in Nearby Active Galaxies. I. Spatially Resolved Mass Outflow Rates for the Seyfert 2 Galaxy Markarian 573, ApJ, 2018, vol. 856, p. 46

Ricci T. V., Steiner J. E., Menezes R. B., IFU spectroscopy of 10 early-type galactic nuclei - II. Nuclear emission line properties, MNRAS, 2014, vol. 440, p. 2442

Ricci T. V., Steiner J. E., Menezes R. B., IFU spectroscopy of 10 early-type galactic nuclei - III. Properties of the circumnuclear gas emission, MNRAS, 2015, vol. 451, p. 3728

Rich J. A., Kewley L. J., Dopita M. A., Galaxy-wide Shocks in Late-merger Stage Luminous Infrared Galaxies, ApJ, 2011, vol. 734, p. 87

Rich J. A., Kewley L. J., Dopita M. A., Composite Spectra in Merging U/LIRGs Caused by Shocks, ApJ, 2014, vol. 781, p. L12

Rich J. A., Kewley L. J., Dopita M. A., Galaxy Mergers Drive Shocks: An Integral Field Study of GOALS Galaxies, ApJS, 2015, vol. 221, p. 28

Richardson C. T., Allen J. T., Baldwin J. A., Hewett P. C., Ferland G. J., Interpreting the ionization sequence in AGN emission-line spectra, MNRAS, 2014, vol. 437, p. 2376

Rigby J. R., Rieke G. H., Donley J. L., et al. Why X-Ray-selected Active Galactic Nuclei Appear Optically Dull, ApJ, 2006, vol. 645, p. 115

Rola C. S., Terlevich E., Terlevich R. J., New diagnostic methods for emission-line galaxies in deep surveys, MNRAS, 1997, vol. 289, p. 419 
Rose M., Tadhunter C., Ramos Almeida C., Rodríguez Zaurín J., Santoro F., Spence R., Quantifying the AGN-driven outflows in ULIRGs (QUADROS) - I: VLT/Xshooter observations of nine nearby objects, MNRAS, 2018, vol. 474, p. 128

Rubin R. H., The Structure and Properties of H II Regions, ApJ, 1968, vol. 153, p. 761

Salim S., Lee J. C., Ly C., Brinchmann J., Davé R., et al. A Critical Look at the MassMetallicity-Star Formation Rate Relation in the Local Universe. I. An Improved Analysis Framework and Confounding Systematics, ApJ, 2014, vol. 797, p. 129

Sánchez S. F., Spatially Resolved Spectroscopic Properties of Low-Redshift Star-Forming Galaxies, ARA\&A, 2020, vol. 58, p. 99

Sarzi M., Shields J. C., et al. The SAURON project - XVI. On the sources of ionization for the gas in elliptical and lenticular galaxies, MNRAS, 2010, vol. 402, p. 2187

Schawinski K., The role of AGN in the migration of early-type galaxies from the blue cloud to the red sequence, AIPC, 2009, vol. 1201, p. 17

Schawinski K., Koss M., et al. Active galactic nuclei flicker: an observational estimate of the duration of black hole growth phases of $\sim 10^{5}$ yr, MNRAS, 2015, vol. 451, p. 2517

Schawinski K., Thomas D., Sarzi M., Maraston C., Kaviraj S., et al. Observational evidence for AGN feedback in early-type galaxies, MNRAS, 2007, vol. 382, p. 1415

Schawinski K., Urry C. M., Virani S., Coppi P., Bamford S. P., Treister E., Lintott C. J., et al. Galaxy Zoo: The Fundamentally Different Co-Evolution of Supermassive Black Holes and Their Early- and Late-Type Host Galaxies, ApJ, 2010, vol. 711, p. 284

Shields G. A., X-ray ionization and the helium abundance in 3C 120, ApJ, 1974, vol. 191, p. 309

Shields J. C., Normal O Stars in Dense Media Generate LINERs, ApJ, 1992, vol. 399, p. L27

Shirazi M., Brinchmann J., Strongly star forming galaxies in the local Universe with nebular He II $\lambda 4686$ emission, MNRAS, 2012, vol. 421, p. 1043 
Shuder J. M., Osterbrock D. E., Empirical results from a study of active galactic nuclei, AJ, 1981, vol. 250, p. 55

Shull J. M., McKee C. F., Theoretical models of interstellar shocks, ApJ, 1979, vol. 227, p. 131

Silk J., Ultraluminous starbursts from supermassive black hole-induced outflows, MNRAS, 2005, vol. 364, p. 1337

Silk J., Rees M. J., Quasars and galaxy formation, A\&A, 1998, vol. 338, p. L1

Simpson J. P., Colgan S. W. J., Cotera A. S., et al. Spitzer IRS Observations of the Galactic Center: Shocked Gas in the Radio Arc Bubble, ApJ, 2007, vol. 670, p. 1115

Singh R., van de Ven G., Jahnke K., Lyubenova M., et al. The nature of LINER galaxies:. Ubiquitous hot old stars and rare accreting black holes, A\&A, 2013, vol. 558, p. 43

Snijders L., Kewley L. J., van der Werf P. P., Mid-Infrared Diagnostics of Starburst Galaxies: Clumpy, Dense Structures in Star-Forming Regions in the Antennae (NGC 4038/4039), ApJ, 2007, vol. 669, p. 269

Sparks W. B., Macchetto F., Golombek D., Imaging Observations of Gas and Dust in NGC 4696 and Implications for Cooling Flow Models, ApJ, 1989, vol. 345, p. 153

Spence R. A. W., Zaurín J. R., Tadhunter C. N., et al. No evidence for large-scale outflows in the extended ionized halo of ULIRG Mrk273, MNRAS, 2016, vol. 459, p. L16

Spinoglio L., Malkan M. A., Infrared Line Diagnostics of Active Galactic Nuclei, ApJ, 1992, vol. 399, p. 504

Spitzer L. J., The Temperature of Interstellar Matter. II, ApJ, 1949, vol. 109, p. 337

Spoon H. W. W., Marshall J. A., Houck J. R., et al. Mid-Infrared Galaxy Classification Based on Silicate Obscuration and PAH Equivalent Width, ApJ, 2007, vol. 654, p. L49

Stanway E. R., Eldridge J. J., Becker G. D., Stellar population effects on the inferred photon density at reionization, MNRAS, 2016, vol. 456, p. 485

Stasińska G., A grid of model nebulae photoionized by a power-law continuum, A\&AS, 1984, vol. 55, p. 15 
Stasińska G., Biases in abundance derivations for metal-rich nebulae, A\&A, 2005, vol. 434, p. 507

Stasińska G., [Ar III]/[O III] and [S III]/[O III]: well-behaved oxygen abundance indicators for HII regions and star forming galaxies, A\&A, 2006, vol. 454, p. L127

Stasińska G., Cid Fernandes R., et al. Semi-empirical analysis of Sloan Digital Sky Survey galaxies - III. How to distinguish AGN hosts, MNRAS, 2006, vol. 371, p. 972

Stasińska G., Schaerer D., What heats the bright H II regions in I ZW 18?, A\&A, 1999, vol. 351 , p. 72

Stasińska G., Vale Asari N., Cid Fernandes R., et al. Can retired galaxies mimic active galaxies? Clues from the Sloan Digital Sky Survey, MNRAS, 2008, vol. 391, p. L29

Storchi-Bergmann T., On the ratio N II/H-alpha in the nucleus of Seyfert 2 and LINER galaxies, MNRAS, 1991, vol. 249, p. 404

Storchi-Bergmann T., Calzetti D., Kinney A. L., Ultraviolet to near-infrared spectral distributions of star-forming galaxies: metallicity and age effects, ApJ, 1994, vol. 429, p. 572

Storchi-Bergmann T., Pastoriza M. G., On the Sulfur and Nitrogen Abundances in the Nucleus of Seyfert 2 and Liner Galaxies, PASP, 1990, vol. 102, p. 1359

Storchi-Bergmann T., Schmitt H. R., Calzetti D., Kinney A. L., Chemical abundance calibrations for the narrow-line region of active galaxies, ApJ, 1998, vol. 115, p. 909

Strateva I., Ivezic Z., Knapp G. R., Narayanan V. K., et al. Color Separation of Galaxy Types in the Sloan Digital Sky Survey Imaging Data, AJ, 2001, vol. 122, p. 1861

Strauss M. A., Weinberg D. H., Lupton R. H., et al. Spectroscopic Target Selection in the Sloan Digital Sky Survey: The Main Galaxy Sample, ApJ, 2002, vol. 124, p. 1810

Strickland D. K., Heckman T. M., Supernova Feedback Efficiency and Mass Loading in the Starburst and Galactic Superwind Exemplar M82, ApJ, 2009, vol. 697, p. 2030

Strom A. L., Steidel C. C., et al. Measuring the Physical Conditions in High-redshift Star-forming Galaxies: Insights from KBSS-MOSFIRE, ApJ, 2018, vol. 868, p. 117 
Strom A. L., Steidel C. C., Rudie G. C., Trainor R. F., et al. Nebular Emission Line Ratios in $\mathrm{z} \cong 2-3$ Star-forming Galaxies with KBSS-MOSFIRE: Exploring the Impact of Ionization, Excitation, and Nitrogen-to-Oxygen Ratio, ApJ, 2017, vol. 836, p. 164

Strömgren B., On the Helium and Hydrogen Content of the Interior of the Stars, ApJ, 1938, vol. 87 , p. 520

Sutherland R. S., Dopita M. A., Cooling Functions for Low-Density Astrophysical Plasmas, ApJS, 1993, vol. 88, p. 253

Sutherland R. S., Dopita M. A., Effects of Preionization in Radiative Shocks. I. Selfconsistent Models, ApJS, 2017, vol. 229, p. 34

Tarter C. B., Salpeter E. E., The Interaction of X-Ray Sources with Optically Thick Environments, ApJ, 1969, vol. 156, p. 953

Tarter C. B., Tucker W. H., Salpeter E. E., The Interaction of X-Ray Sources with Optically Thin Environments, ApJ, 1969, vol. 156, p. 943

Thomas A. D., Groves B. A., et al. A Physically based Model of the Ionizing Radiation from Active Galaxies for Photoionization Modeling, ApJ, 2016, vol. 833, p. 266

Thomas A. D., Kewley L. J., Dopita M. A., Groves B. A., Hopkins A. M., Sutherland R. S., The mass-metallicity relation of local active galaxies, ApJ, 2019, vol. 874, p. 100

Tinsley B. M., Evolution of the Stars and Gas in Galaxies, ApJ, 1968, vol. 151, p. 547

Tremonti C. A., Heckman T. M., Kauffmann G., Brinchmann J., Charlot S., White S. D. M., et al. The Origin of the Mass-Metallicity Relation: Insights from 53,000 Starforming Galaxies in the Sloan Digital Sky Survey, ApJ, 2004, vol. 613, p. 898

Trouille L., Barger A. J., The OPTX Project. IV. How Reliable is [O III] as a Measure of AGN Activity?, ApJ, 2010, vol. 722, p. 212

Trump J. R., Impey C. D., Taniguchi Y., Brusa M., Civano F., et al. The Nature of Optically Dull Active Galactic Nuclei in COSMOS, ApJ, 2009, vol. 706, p. 797

Vale Asari N., Cid Fernandes R., Stasińska G., et al. The history of star-forming galaxies in the Sloan Digital Sky Survey, MNRAS, 2007, vol. 381, p. 263 
Vale Asari N., Couto G. S., Cid Fernandes R., et al. Diffuse ionized gas and its effects on nebular metallicity estimates of star-forming galaxies, MNRAS, 2019, vol. 489, p. 4721

Vale Asari N., Stasińska G., Morisset C., Cid Fernandes R., BOND: Bayesian Oxygen and Nitrogen abundance Determinations in giant H II regions using strong and semistrong lines, MNRAS, 2016, vol. 460, p. 1739

van Zee L., Haynes M. P., Salzer J. J., Element enrichment and stellar populations of gas-rich low surface brightness dwarf galaxies, AJ, 1997, vol. 114, p. 6

Veilleux S., Cecil G., Bland-Hawthorn J., Galactic Winds, ARA\&A, 2005, vol. 43, p. 769

Veilleux S., Osterbrock D. E., Spectral classification of emission-line galaxies, ApJS, 1987, vol. 63 , p. 295

Veilleux S., Shopbell P. L., Rupke D. S., et al. A Search for Very Extended Ionized Gas in Nearby Starburst and Active Galaxies, AJ, 2003, vol. 126, p. 2185

Viegas S. M., Prieto M. A., Probing photoionization models in two well-studied extended emission-line regions : Centaurus A and 3C 227, MNRAS, 1992, vol. 258, p. 483

Viegas-Aldrovandi S. M., Gruenwald R. B., The LINERs Revisited, ApJ, 1990, vol. 360, p. 474

Viironen K., Delgado-Inglada G., Mampaso A., et al. The S2N2 metallicity calibrator and the abundance gradient of M33, MNRAS, 2007, vol. 381, p. 1719

Vila-Costas M. B., Edmunds M. G., The nitrogen-to-oxygen ratio in galaxies, and its implications for the origin of nitrogen, MNRAS, 1993, vol. 265, p. 199

Villar-Martín M., Binette L., Fosbury R. A. E., The effects of resonance scattering and dust on the UV line spectrum of radio galaxies, A\&A, 1996, vol. 312, p. 751

Villar-Martín M., Tadhunter C., Clark N., The ionization mechanism of the extended gas in high redshift radio galaxies: shocks or AGN photoionization?, A\&A, 1997, vol. 323, p. 21

Voit G. M., Infrared Fine-Structure Line Diagnostics of Shrouded Active Galactic Nuclei, ApJ, 1992, vol. 399, p. 495 
Voit G. M., Donahue M., Self-irradiated Cooling Condensations: The Source of the Optical Line Emission from Cooling Flows, ApJ, 1990, vol. 360, p. L15

Wang J.-M., Ge J.-Q., et al. Star Formation in Self-gravitating Disks in Active Galactic Nuclei. I. Metallicity Gradients in Broad-line Regions, ApJ, 2011, vol. 739, p. 3

Weaver K. A., Meléndez M., Mushotzky R. F., Kraemer S., Engle K., Malumuth E., et al. Mid-infrared Properties of the Swift Burst Alert Telescope Active Galactic Nuclei Sample of the Local Universe. I. Emission-line Diagnostics, ApJ, 2010, vol. 716, p. 1151

Weedman D. W., High-Velocity Gas Motions in Galactic Nuclei, AJ, 1970, vol. 159, p. 405

Weedman D. W., Seyfert galaxies, ARA\&A, 1977, vol. 15, p. 69

Weedman D. W., Feldman F. R., Balzano V. A., Ramsey L. W., Sramek R. A., Wuu C.-C., NGC 7714 - The prototype star-burst galactic nucleus, ApJ, 1981, vol. 248, p. 105

Whittle M., The narrow line region of active galaxies - II. Relations between O III profile shape and other properties, MNRAS, 1985, vol. 213, p. 33

Whittle M., Virial and Jet-induced Velocities in Seyfert Galaxies. II. Galaxy Rotation as Virial Parameter, ApJ, 1992, vol. 387, p. 109

Wild V., Heckman T., Charlot S., Timing the starburst-AGN connection, MNRAS, 2010, vol. 405 , p. 933

Williams R. E., The Ionization and Thermal Equilibrium of a Gas Excited by Ultraviolet Synchrotron Radiation, ApJ, 1967, vol. 147, p. 556

Wilson A. S., Willis A. G., Radio structures of Seyfert galaxies. I., ApJ, 1980, vol. 240, p. 429

Wright E. L., A Cosmology Calculator for the World Wide Web, PASP, 2006, vol. 118, p. 1711

Yan R., Blanton M. R., The Nature of LINER-like Emission in Red Galaxies, ApJ, 2012, vol. 747 , p. 61

Yan R., Ho L. C., Newman J. A., Coil A. L., et al. AEGIS: Demographics of X-ray and Optically Selected Active Galactic Nuclei, ApJ, 2011, vol. 728, p. 38 
York D. G., Adelman J., Anderson J. E., Scott F. A., Annis J., et al. The Sloan Digital Sky Survey: technical summary, ApJ, 2000, vol. 120, p. 1579

Yuan F., Narayan R., On the Nature of X-Ray-Bright, Optically Normal Galaxies, ApJ, 2004, vol. 612 , p. 724

Zamorani G., Henry J. P., Maccacaro T., Tananbaum H., Soltan A., Avni Y., et al. X-ray studies of quasars with the Einstein Observatory II, ApJ, 1981, vol. 245, p. 357

Zanstra H., On radiative equilibrium and radiation pressure in a stationary nebula with an appendix by J. H. de Jong, BAN, 1951, vol. 11, p. 359

Zhang K., Yan R., Bundy K., Bershady M., Haffner L. M., Walterbos R., et al. SDSSIV MaNGA: the impact of diffuse ionized gas on emission-line ratios, interpretation of diagnostic diagrams and gas metallicity measurements, MNRAS, 2017, vol. 466, p. 3217

Zhang Z. T., Liang Y. C., Hammer F., Modelling the narrow-line regions of active galaxies in the Sloan Digital Sky Survey - I. Sample selection and physical conditions, MNRAS, 2013, vol. 430, p. 2605 
Appendix 

Appendix A

\section{More complex models for AGN}

In Section 3.3.4, we have seen that schematic photoionization models are not sufficient to represent the observational data in the standard diagnostic diagrams. The physical explanation for that is that gaseous nebulae are very complex environments $\mathrm{Ho}, 2008$; Kakkad et al., 2018), and each line depends on the density, temperature, incident radiation field, ionization parameter, etc. (Meléndez et al., 2014, Kewley et al., 2019). Hence, if the diff lines are not produced cospatially, single-zone photoionization models will not be able to match many of the lines simultaneously, and multicomponent models that incorporate a range of densities are required ( $\mathrm{Ho}, 2008)$.

The model H II regions are being refined, as discussed in Section 3.3.1, by the development of the stellar atmospheres, stellar tracks, and the consequent stellar SEDs generated with stellar evolutionary synthesis codes. A better definition of atomic data is also in course in laboratories (Kewley et al., 2019). We also emphasize here the need of addressing pAGB stellar populations, since the modelling of such objects with evolutionary population synthesis codes is still uncertain (Stasińska et al., 2008).

As for AGN models, approaches beyond simple photoionization models with power-law SEDs have been proposed for the last 40 years. The main issues are: (i) the inability to provide the uniformity in emission line ratios that is usually observed without making arbitrary and possibly unphysical assumptions (Groves et al., 2004), (ii) the inability in reproducing weak high excitation lines such as [Nev] $\lambda 3426, \mathrm{C}$ IV $\lambda \lambda 1549, \mathrm{C}$ III $] \lambda \lambda 1909$, [Fe VII] $]$ 6087, and [Fe x] 66375 (see Stasińska, 1984, Ferland and Osterbrock, 1986), (iii) the temperature problem, with the prediction of too low electronic temperatures (see $\mathrm{Ho}$, 2008: Dopita et al., 2013), (iv) the prediction of too small ranges in the $\mathrm{He} \mathrm{II} / \mathrm{H} \beta$ line ratio (see Binette et al. 1996, 1997), (v) the absence of a description of structures that 
are identified by data with high spatial resolution, as ionization cones, knots, filaments, and strands of ionized gas (Ferguson et al. 1997), and (vi) the inability to describe the correlation between the line widths of optical forbidden emission lines with the critical density and the ionization potential of such lines (Filippenko and Halpern, 1984; Ferguson et al., 1997).

We will present the most relevant proposals of AGN models in this Section, which are the matter- and ionization-bounded clouds (Binette et al., 1996, 1997), the Local Optimally emitting Clouds (LOC, Baldwin et al., 1995; Ferguson et al., 1997), shock models Viegas-Aldrovandi and Gruenwald, 1990; Dopita and Sutherland, 1995, 1996; Contini, 1997; Dors et al., 2021), and dusty, radiation pressure-dominated photoionization models (Dopita et al., 2002; Groves et al., 2004). Composite photoionization models have also been proposed, considering the combination of more than one density in the photoionization models and giving different weights to each of the components Stasińska, 1984; Komossa and Schulz, 1997). However, we will treat such kind of approach by describing the matter- and ionization-bounded clouds, which is a two zone approximation, and LOC, which considers a whole family of photoionized clouds of different densities and distances from the central AGN (Dopita et al., 2002; Meléndez et al., 2011). Though such approaches solve some problems, they maintain the simplistic perspective of the simple photoionization models, without physical details on the distribution and behavior of the proposed components (Veilleux and Osterbrock, 1987). In this Section, we will not consider the effects of cosmic rays and DIG, though they might be relevant, they have not yet been robustly included in theoretical models (Kewley et al., 2019). It is also worth pointing out that the strategies adopted by the different models are not necessarily contradictory, and might be combined in some of the cases.

\section{A.1 Matter- and ionization-bounded clouds}

The combination of matter- and ionization-bounded (or radiation-bounded) clouds to model AGNs was proposed by Viegas and Prieto (1992) and improved by Binette et al. (1996, 1997) in order to solve the issues that photoionization models did not address correctly.

The matter-bounded component is responsible for most of the high excitation lines and 
by the coronal lines, and it is geometrically smaller than the depth needed to absorb all incident ionizing photons. In the models of Binette et al. (1996, 1997), it was considered that the matter-bounded clouds had to be sufficiently thick to reprocess $\sim 40 \%$ of the ionizing photons to which they were exposed. On the other hand, the ionization-bounded component would be a region much more opaque to the ionizing radiation, where low-tointermediate excitation lines are produced. Such clouds would present a low ionization parameter, and they are mainly exposed to the radiation spectrum that exits the matterbounded clouds, more than directly receiving the radiation field produced by the central power source. Hence, the geometrical thickness of the clouds is the parameter that determines whether the photoionization model considered is matter- or ionization-bounded (Dors et al., 2021), but they can also be determined by a combination of high density and low ionization for radiation-bounded clouds and low density and high ionization for matter-bounded components (Meléndez et al., 2011).

Binette et al. (1996) proposes a sequence of photoionization calculations where, instead of varying the ionization parameter as it is often done, the varying parameter is the solid angle ratio occupied by matter-bounded clouds relative to the ionization-bounded component. The ionization parameter sequence implies that the [O III] emitting zone becomes progressively more important relative to the $[\mathrm{O} \mathrm{I}]$ emitting region as $U$ increases. By successfully describing the observed trends in a similar way to what the ionization parameter sequence proposes, it is argued that the apparent variations in excitation represent different proportions of two different populations of line emitting clouds, with the high excitation matter-bounded clouds becoming more dominant (in number or solid angle) over the ionization-bounded clouds as the ratio increases.

There are mainly two physical distributions of the components proposed by this model, as shown in Figure A.1. The top panel shows a perspective where the matter- and the ionization-bounded components are distinguished by the direct incidence of the radiation field emitted by the central source, while the bottom panel indicates a model where these would be different parts intrinsic of the same clouds, where the matter-bounded component would be turned towards the central source, in such a way that the matter-bounded component would be the low density 'halo' of a cloud and the ionization-bounded component would be its high density core.

In Binette et al. (1997), the effects of radiation pressure are considered in order to 


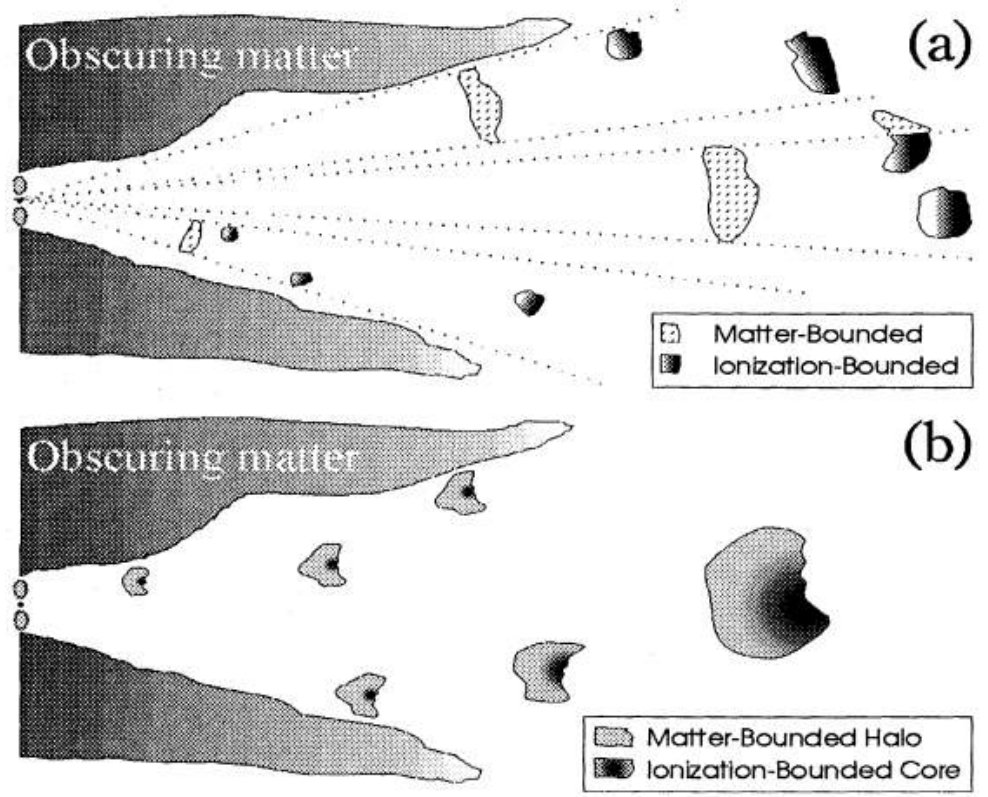

Figure A.1: Figure 4 from Binette et al. (1996), showing the cartoons of two possible geometrical distributions of matter-bounded and ionization-bounded components. The luminosity of any component is essentially governed by the solid angle it covers at the central UV source. The inner clouds (NLR) are denser and smaller than those at larger radii, where the cloud sizes become sufficiently large that individual components become resolvable (ENLR). In the models presented on the paper, the ionization-bounded clouds are of lower excitation mostly because they see an ionizing continuum filtered by the matterbounded component. (a) The matter-bounded clouds are closer to the ionizing source and shadow more distant ionization-bounded clouds which see a more dilute radiation field and hence have lower ionization parameter. Supposing a planar or cylindrical distribution of obscuring material, the relative numbers of visible ionization- and matter-bounded clouds can vary considerably according to the perspective of the observer. (b) The ionization-bounded component is associated with the dense core (higher pressure) of a gas cloud surrounded by a tenuous matter-bounded evaporating halo.

explain the systematic blueshift that is often observed in coronal lines, by the strong density gradient that it generates in the photoionized structure and that is responsible for the acceleration of the matter-bounded gas. The radiation pressure inclusion in the one dimensional photoionization code is described within the paper. It relies on the idea that, if a cloud of neutral gas is suddenly irradiated by an external ionizing radiation field, the cloud surface would be ionized and an ionization front would travel into the cloud. The photoionized layer would be heated to $\sim 10^{4} \mathrm{~K}$, resulting in a substantial overpressure which would drive an outward flow of the gas whose density decreases with time. The radiation pressure of the photon field on the gas will tend to stop this outward flow. Hence, the gas would be maintained in its position by a balance between the gas pressure gradient (directed outward) and the force due to the gradual absorption of the incident ionizing photon field (directed inward). 
This model of two zone approximation, with matter- and ionized-bounded clouds that have different properties, is considered in some more recent models to explain the behavior of lines from the same species that have different ionization levels and other issues, as seen in Baskin and Laor (2005), Meléndez et al. (2011), and others. Cerqueira-Campos et al. (2021) uses this approach to justify the variety of coronal lines observed in Coronal Line Forest AGNs, arguing that such objects are dominated by matter-bounded clouds. However, one of the issues is the scarce definition of the geometry in such models, that becomes a free parameter, and another problematic point is the assumed difference in the pressure between the two types of clouds (or the cloud and inter-cloud components), which is not physically explained (Dopita et al., 2002).

\section{A.2 Local optimally emitting clouds}

The "Locally Optimally emitting Clouds" (LOC) model considers a combination of photoionized clouds of different densities at different distances from the central ionizing source (Feltre et al. 2016). The intention of such approach is to provide more accurate models to describe quasar (Baldwin et al., 1995) and AGN spectra Ferguson et al. (1997).

The NLR models should be in agreement with the observed correlation between the line width of optical forbidden lines and the critical density and ionization potential of those lines, which indicates that the photoionized region must be stratified and the lines are most efficiently emitted near their critical densities (Filippenko and Halpern, 1984). Thus, the LOC models are a quantitative formulation of such hypothesis.

This model is proposed by Baldwin et al. (1995) and Ferguson et al. (1997), where a very large grid of photoionization models is run considering different densities and distances from the central continuum source. They notice that, for each analyzed line, there was a narrow range of such variables that would result in efficient emission, corresponding to optimally selected clouds. Hence, the spectra of different types of AGNs can be reproduced by adding together the full family of clouds, with an appropriate covering fraction distribution.

Therefore, this general approach shows that there is a powerful selection effect operating in the NLR environment, in such a way that a random mix of cloud properties is able to reproduce the spectra of typical AGNs and quasars. This selection effect consists in the fact that we observe the lines that are emitted in the clouds that are best able to produce 
such lines. Hence, as long as there are enough clouds at the correct radius and with the correct gas density to efficiently form a given line, the line will be emitted with a relative strength very similar to what is usually observed. This would indicate that the AGN spectrum is a natural consequence of the ability of several different clouds to reprocess the underlying continuum, favoring a chaotic environment with no preferred values for the pressure, the gas density, or the ionization parameter. The parameters inferred from the spectra of AGN are thus associated with averages of the global properties, rather than about the details of individual clouds.

By coupling the LOC scenario with a Keplerian gravitational velocity field, Ferguson et al. (1997) explains the correlation between the observed line widths and the critical density of the forbidden lines. Hence, the dominant velocity field associated with the line widths diminishes with increasing distance from the nucleus, and the expectation is that the lines that are emitted optimally at smaller distances and with higher densities will present broader line widths in comparison to the lines that are optimally emitted at larger distances and with lower densities.

As for the presence of dust, the results of Ferguson et al. (1997) indicate that there might be a region closer to the central source with high ionization where the dust grains would not survive the intense radiation field (see the sublimation processes discussed by Laor and Draine, 1993), but the further regions where neutral gas dominates are more likely to be dusty. This result comes from the comparison of dusty and dust-free photoionization models with observational spectra, and once again favours the hypothesis that the NLR consists in a variety of clouds at different conditions. Such hypothesis is in agreement with the observational facts that low ionization lines such as the UV line of Mg II, the optical line of [CaII], and the IR line of [Fe II] indicate the presence of dust, while strong coronal lines suggest a dust-free environment. These regions with and without dust thus coexist at different distances from the central source.

Therefore, the LOC Models are successful to reproduce quasar and AGN NLR spectra by assuming that the emitting region consists of clouds with a wide range of gas densities and distances from the central ionizing source. The models can reproduce the expected line-ratio range from standard diagnostic diagrams and the predicted $\mathrm{H} \beta$ equivalent width. They also address the temperature problem by reproducing both the low density expected from the $[\mathrm{S} \mathrm{II}]$ line ratio and the high temperature from the [O III] line ratio. The issues 
with regard to the relation between the observed line widths and the critical densities of each line and with the presence of lines that indicate both the presence and absence of dust are also disentangled. However, though the models fit the observed spectral quite well, the geometry of the clouds and their relative contributions to the expected emission remain free parameters and are poorly constrained (Dopita et al., 2002).

\section{A.3 Shocks}

The presence of shock waves in many galactic nuclei is undeniable. Shocks are prevalent in several astronomical phenomena, as for example AGN jets and outflows, supernova explosions, stellar winds, cloud collisions (from galaxy interactions, mergers, and other phenomena), ram-pressure stripping, etc. (Yan and Blanton, 2012, Kewley et al., 2019). They can provide collisional ionization or photoionization by the shock itself (Binette et al., 1996), and they also interfere on several properties that the emission lines depend on, such as the gas density, the temperature downstream, and the cooling rates throughout the clouds (Dors et al. 2021). In the case of star-forming and interacting galaxies, the stratification structures that strong winds produce can be visible, and in the case of AGNs we also have signatures that shocks are occurring when ionization cones or spherically symmetric bubbles of ionized gas are seen in the vicinity of the nucleus (Dopita and Sutherland, 1995).

The main parameters of shock models are the shock velocity, the magnetic field strength, the density, and the metallicity. Shock excitation can interfere on both low- and highionization lines, depending on the shock velocity. Fast radiative shock models can reproduce strong high-ionization lines (Allen et al., 1998, 2008) and models considering slow shocks can reproduce the relatively weak high-ionization lines and the strong low-ionization species (Rich et al., 2011, 2015). Such difference in the preferred emitted lines is due to the photoionizing precursor produced by fast shocks, that generates significant ionization in the front of the shock (Kewley et al., 2019). The presence of a wide range of shock velocities in a galactic nucleus would explain the observed differences on the widths of distinct lines (Yan and Blanton, 2012).

Interstellar shock models have been developed since the 1970s, with relevant works as Dopita (1976, 1977), Raymond (1979), and Shull and McKee (1979). These have offered 
much of the theoretical basis that provided further shock models to synthesize NLR AGN spectra.

Models that consider pure shock heating and their ionization effects have been conducted by Sutherland and Dopita (1993) and Dopita and Sutherland (1995, 1996), with the argument that the NLRs of AGNs would be predominantly shock excited by flows of mechanical energy. They propose radiative steady-flow shock models supported by magnetic pressure in the photoionization/recombination zone of the shock (Dopita and Sutherland, 1996) to reproduce the line ratios of some diagnostic diagrams (Dopita and Sutherland, 1995). The models are able to reproduce the optical narrow emission lines typical from Seyferts and LINERs by setting conditions of high and low densities, respectively. Seyfert 1.5 and 2 nuclei would be associated with jet-powered outflow, while LINERs would be linked to accretion in the case of cooling flows, and some combination of these two processes could also be taking place in AGNs. The main difference between LINER-like and Seyfert-like objects would be the amount of available gas: gas-rich environments associated with Seyferts would have material beyond the shock to absorb the EUV photons produced in the cooling zone of the shock in an extended preshock H II region, while objects producing LINER-like spectra are associated with the absence of the precursor H II region of the shock. Dopita and Sutherland (1995) argues that the line ratio profile of (some) LINERs can be explained solely by the collisional excitation of the post-shock medium, without the need to consider a central ionizing source. The shock velocity, for producing a stronger and harder EUV photon field, plays the role of both the ionization parameter and the spectral index that are considered in photoionization models of AGNs, while the magnetic parameter increases the ionization parameter in the cooled shock plasma. With the proposed models, the problem of the electron temperatures observed being systematically higher than predicted in photoionization models is solved. The proposed models are able to reproduce the observed line widths of Seyferts and LINERs and also to address some issues that the photoionization models could not simulate, such as the correlations between radio power and luminosity (de Bruyn and Wilson, 1964) or line width (Wilson and Willis, 1980) that are found in some objects (though it was still not clear if such properties were global to all AGN NLRs). However, the fast shock models have their limitations with regard to the difficulty in reproducing strong coronal lines (such as [Fe VII], [Fex], [S VIII], [S XII], $[\mathrm{Si} \mathrm{VI}]$ and [Si VII]), the large outflow velocities inferred in the [O III] line in some objects, 
and the narrow lines of most of the Seyferts, which are consistent with line broadening associated with galactic rotation more than to shocked outflows (Dopita et al., 2002).

Models that consider the contribution of both collisional effects from shocks and photoionization from an active central source are proposed by Contini and Viegas-Aldrovandi (1989), Viegas-Aldrovandi and Gruenwald (1990), Contini (1997), Inskip et al. (2002), and other works, with the argument of being more realistic than simple models that assume only one of the two types of ionization that are observed in galactic nuclei. Such composite models are used both to simulate AGNs or starburst galaxies (see Congiu et al. 2017; Contini, 2019). Dors et al. (2021) applies composite models to describe a sample of Seyfert 2 nuclei, and finds that the FWHM of optical emission lines is a good tracer of shock velocities. The photoionization source is treated as a power-law, as usual, and the shocks contribute to the collisional ionization and heating of the gaseous nebulae; dust is included in such models. One of the results is that the range of $\mathrm{O} / \mathrm{H}$ abundances found for composite models is much smaller than for pure photoionization models, which indicates the strong dependence of oxygen emission lines on shock parameters as the shock velocity, rather than on the $\mathrm{O} / \mathrm{H}$ abundance.

As we have seen in Section 1.2.2, the standard optical diagnostic diagrams are not ideal for separating the excitation sources in the cases where shock emission is suspected (Kewley et al., 2019), and parameters such as the velocity dispersion (D'Agostino et al., 2019) and UV emission lines (Dopita and Sutherland, 1995; Allen et al., 1998, 2008) can be useful. The shock models, when applied to the traditional diagnostic diagrams, cover large areas and encompass all distinct loci (e.g., Allen et al., 2008; Ho et al., 2014).

Another important constraint is that, mainly for weak AGN, the presence of shocks does not imply that such mechanism is the prevalent source of ionizing photons in the observed region (Belfiore et al., 2016). Though Dopita and Sutherland (1995) argue that the advantage of the shock model is that it provides a natural link between the spectra characteristics, the surface brightness, and the dynamics of the NLRs, several important parameters and effects remain without further explanation, as for example the finite shock age and the limited gas supply through the shock.

Moreover, the postshock cooling zone of shock models attains a much higher equilibrium electron temperature than what is predicted for a photoionized plasma. As a consequence, it is expected that shocked gas produces a higher excitation spectrum (which is most 
readily discernible in the UV) than photoionized gas. However, this fast-shock scenario is inconsistent with several observations of high excitation lines such as CIV $\lambda 1549$ and He $\lambda 1640$ (e.g., Barth et al., 1996, 1997; Maoz et al., 1998).

Therefore, though it is certain that shocks are present in most galaxies and very important in cases where jet-driven outflows can lead to observed high velocities, they are not likely the dominant power source to produce emission lines in galactic nuclei (Yan and Blanton, 2012). In most of the cases, the contribution from shock excited clouds is either indistinguishable or negligible compared the contribution from photoionized clouds (Groves et al., 2004). Sarzi et al. (2010) supports this idea after analyzing low circular velocities and low velocity dispersions in AGNs, with a lack of morphological correlation between line emission structure and line ratio structure, and also a flat line width distribution. Hence, some of the arguments that Dopita and Sutherland (1995, 1996) based on are probably local, and not global properties of NLRs, and more robust correlation between line ratios and velocities would be necessary to make such relations generalizable. Shocks definitely exist in several situations, but presenting proper conditions for shocks to occur does not mean that shocks are directly responsible for the ionization of the gas (Yan and Blanton, 2012). The remaining issue relies on the quantification of the effects of such shocks and their balance with regard to photoionization to better understand the activity on galactic nuclei $(\mathrm{Ho}, 2008)$.

\section{A.4 Dusty, radiation pressure-dominated photoionization}

The last type of complex model that intends to reproduce AGN spectra presented in this Section is the dusty, radiation pressure-dominated photoionization model. These models were proposed by Dopita et al. (2002) and Groves et al. (2004), and they consist on clouds of a single type, but including dust and being dominated by radiation pressure. Such approach provides good agreement over a wide range of ionization parameters Richardson et al. 2014).

The inclusion of radiation pressure to photoionization models of the emission line regions of AGNs was first proposed by Elitzur and Ferland (1986). This affects the internal density structure, mainly at high ionization parameter conditions, which means that this model is more adequate to describe Seyferts than LINERs. The radiation pressure acting 
on dust can produce fast outflows that are observed in many AGNs.

The presence of dust affects the temperature structure of the photoionized plasma via photoelectric heating, and it can compete with the gas in the absorption of ionizing photons. Dopita et al. (2002) gives details about the several ways that dust grains can be destroyed (such as high temperatures and other effects relevant at smaller scales) and also about the elements that can be depleted into dust and that are observed in coronal lines (such as iron and silicon); the coronal gas, thus, must be a dust-free environment to explain its observed emission lines. Hence, the proposed models consist of a dusty, radiationpressure dominated region surrounding a photoevaporating molecular cloud, which in turn is surrounded by a coronal halo within which the dust has been largely destroyed. With this distribution of different gaseous conditions, the models can explain the coexistence of low-, intermediate-, and coronal ionization zones within a single self-consistent physical structure. Figure A.2 is the same as Figure 1 from Dopita et al. (2002), where the model is schematized.

The introduction of such models by Dopita et al. (2002) compares the distribution of different photoionization models (constant pressure versus constant density and dust-free versus dusty) on diagnostic diagrams to constrain their viability. The favoured models in the comparison with observational data are the ones that contain a strong density gradient increasing towards the ionization front (isobaric) and are characterized by strong photoelectric heating by the dust, with the dominant absorption of the ionizing continuum due to the dust. In such models, the gas pressure close to the ionization front is determined by the radiation field. Under the mentioned conditions, the models can reproduce line spectra consistent with observations (with the co-existence of low-ionization zones and a coronal region), solve the temperature problem by providing a higher electron temperature than standard photoionization models, and propose a mechanism to explain the observed outflows and the broad blueshifted [O III] that are observed in some AGNs.

The work from Groves et al. (2004) focuses on the reproduction of a spectral uniformity that is observed for several Seyfert nuclei. The effects of the radiation pressure acting upon dust provides a controlling factor in moderating the density, the excitation, and the surface brightness of photoionized emission line structures so they are confided in the specific range in which they are often observed. Hence, the proposed models not only reproduce the observed spectra, but also reproduce the clustering of Seyfert NLRs with similar parameters 


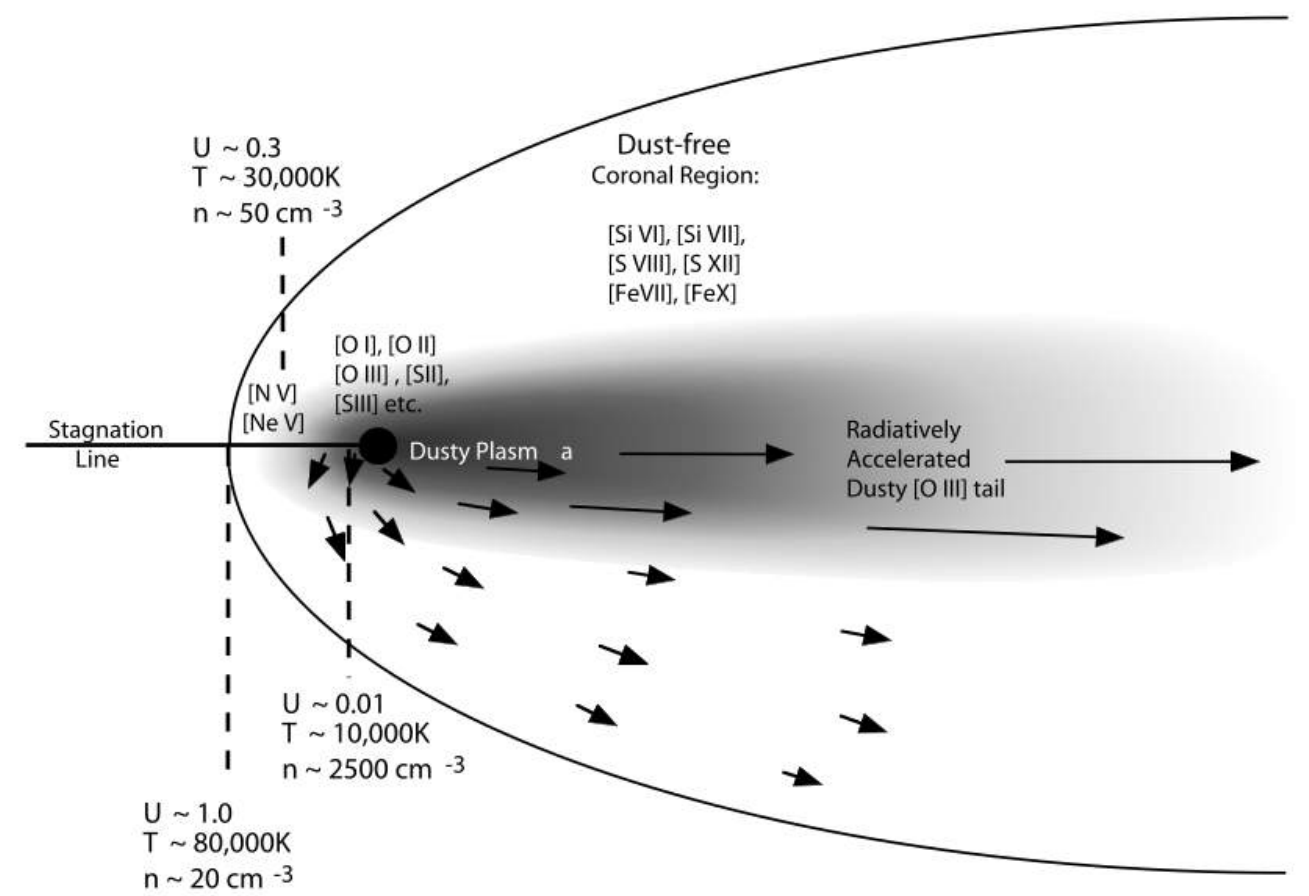

Figure A.2: Figure 1 from Dopita et al. (2002), showing the cartoon of the dusty, radiation pressuredominated photoionization models. The filled circle at the center represents the photoevaporating, dusty molecular cloud, that is surrounded by the dusty, photoevaporated plasma, from which both the low- (close to the cloud) and intermediate-ionization (close to the coronal region) lines are emitted. This plasma is itself surrounded by the dust-free coronal region. The stagnation line indicates the region which is modeled in the paper, where the radiation pressure gradient is matched by the gas pressure gradient, in such a way that the photoionization models can be considered as static. The parameters marked by the dashed lines indicate typical values for each region of a NLR cloud found in NGC 1068.

in their locus at the diagnostic diagrams, as also expected from observations. This provides a physical model that explains the small range in the values of the variables without denying the work based on standard photoionization models, matter- and ionization-bounded clouds, or LOC, but actually improves the usage of photoionization models by providing high- and low-ionization lines without requiring the combination of clouds with different densities and ionization states. Groves et al. (2004) argues that the dusty, radiation pressure-dominated models can explain the assumptions from the other models, with a match in the geometrical distribution proposed in Figures A.2 and the second panel of Figure A.1. The dusty model provides both the low-ionization component dominated by ionization and the high-ionization matter-bounded component within a single model by the self-shielding, so that the high-ionization component sees the direct spectrum while the low-ionization component near the ionization front sees the self absorbed spectrum, and 
they also explain the differences in pressure between the two components by the effects of the radiation pressure on dust. Moreover, the dusty, radiation pressure-dominated models also do not prove the shock excitation models incorrect, since they can be noticeable and relevant in some cases where jets or strong outflows are present in AGNs.

However, though this theoretical approach seems to solve several issues, it is valid only for the NLR of Seyfert galaxies. Netzer (2009) shows that radiation pressure force is not important in the BLR of $0.1 \leq z \leq 0.2$ AGNs with $L_{5100}=10^{42.8-44.8} \mathrm{erg} \mathrm{s}^{-1}$, by the comparison of the supermassive black hole mass estimated via two independent methods. Belfiore et al. (2016) find that, from the observed range of ionization parameter, radiation pressure is probably negligible for LINERs.

Some observational works show that radiation pressure is relevant to the ENLR of Seyfert galaxies. Dopita et al. (2014) finds that the models that include radiation pressure more adequately describe the observed spectrum of the nucleus of NGC 5427. Considering a sample of Seyfert galaxies, Davies et al. (2016) finds that the mixing sequence between star-forming regions and AGN reflects the balance between gas pressure and radiation pressure in the ENLR. In the sample, radiation pressure is relevant, but not throughout the whole observed region. As for Bianchi et al. (2019), the density and ionization distribution of obscured AGNs is explained by the radiation pressure compression scenario, justifying that this mechanism is dominant in X-ray NLRs of Seyfert galaxies. Therefore, radiation pressure is dominant in several cases, but a statistical study is necessary in order to determine if it is ubiquitous in Seyfert NLRs. 


\section{Appendix B}

\section{Aperture effect}

Storchi-Bergmann (1991) shows that the [N II]/H $\alpha$ ratio of Seyfert 2s ans LINERs depends systematically on the aperture size or the source distance. Larger apertures include more extranuclear emission. This will be observed as a contamination of the spectrum of nuclear sources with lower values for $[\mathrm{N}$ II $] / \mathrm{H} \alpha$. Such effects might interfere in the classification of a galactic nuclei according to the diagnostic diagrams, with AGN emission being diluted by nearby H II regions.

If this contamination affects our sample, since SDSS considers a fixed aperture size, the distance to the sources should influence the classification. In Figure B.1, we plot the $[\mathrm{N} \mathrm{II}] / \mathrm{H} \alpha$ ratio versus their redshift (similar to Figure 15 from Ho et al. 1997).

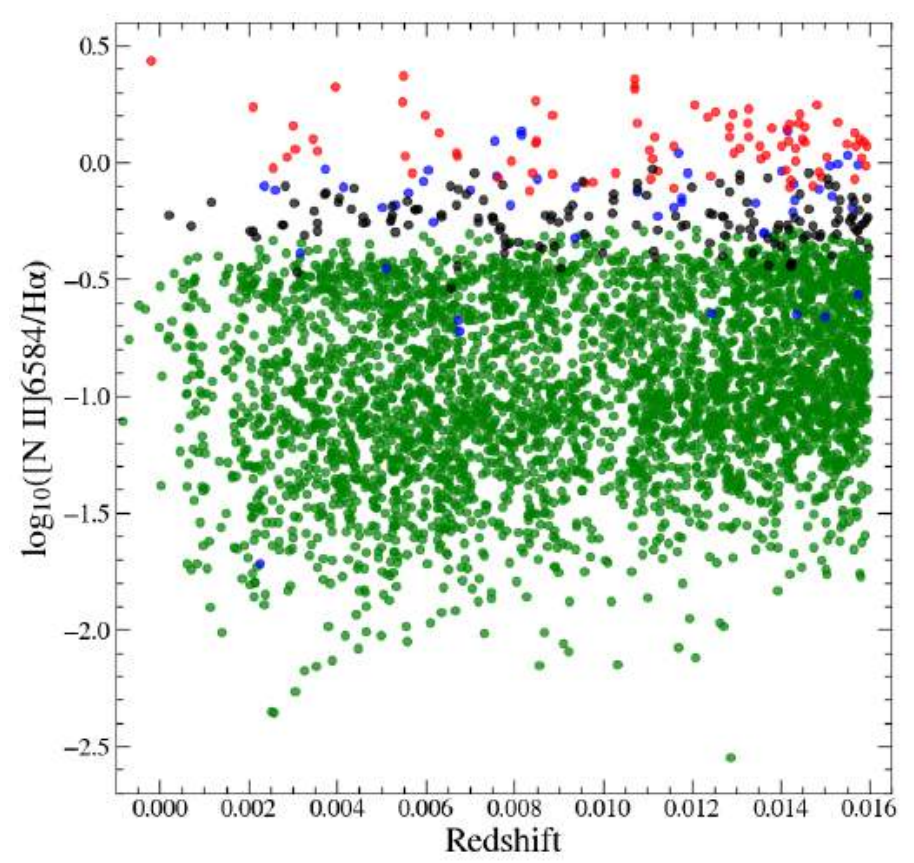

Figure B.1: [N II]/H $\alpha$ versus redshift. Points in green are star-forming nuclei, in red are LINERs, in blue are Seyferts, and in black are transition objects, according to Cid Fernandes et al. (2010). 
As expected for the range $z<0.016$, there are more objects with higher redshift. However, no particular trend is observed in the sense of having more transition objects and $\mathrm{H}$ II regions for larger source distance, or on having lower values of $[\mathrm{N} \mathrm{II}] / \mathrm{H} \alpha$ with increasing redshift.

Hence, we conclude that our redshift cut considers objects close enough for the contamination effects in the nuclear spectra are not significant. 